\title{
Moduli of Stable Parabolic Connections, Riemann-Hilbert Correspondence and Geometry of Painlevé Equation of Type VI, Part I
}

\author{
Dedicated to Professor Kyoichi Takano on his 60th birthday
}

By

Michi-aki INABA*, Katsunori IwASAKI** and Masa-Hiko SAITO***

\begin{abstract}
In this paper, we will give a complete geometric background for the geometry of Painlevé VI and Garnier equations. By geometric invariant theory, we will construct a smooth fine moduli space $M_{n}^{\boldsymbol{\alpha}}(\mathbf{t}, \boldsymbol{\lambda}, L)$ of stable parabolic connections on $\mathbf{P}^{1}$ with $\log$ arithmic poles at $D(\mathbf{t})=t_{1}+\cdots+t_{n}$ as well as its natural compactification. Moreover the moduli space $\mathcal{R}\left(\mathcal{P}_{n, \mathbf{t}}\right)_{\mathbf{a}}$ of Jordan equivalence classes of $S L_{2}(\mathbf{C})$-representations of the fundamental group $\pi_{1}\left(\mathbf{P}^{1} \backslash D(\mathbf{t}), *\right)$ are defined as the categorical quotient. We define the Riemann-Hilbert correspondence $\mathbf{R H}: M_{n}^{\boldsymbol{\alpha}}(\mathbf{t}, \boldsymbol{\lambda}, L) \longrightarrow \mathcal{R}\left(\mathcal{P}_{n, \mathbf{t}}\right)_{\mathbf{a}}$ and prove that $\mathbf{R H}$ is a bimeromorphic proper surjective analytic map. Painlevé and Garnier equations can be derived from the isomonodromic flows and Painlevé property of these equations are easily derived from the properties of $\mathbf{R H}$. We also prove

\footnotetext{
Communicated by T. Kawai. Received March 15, 2005.

2000 Mathematics Subject Classification(s): 34M55, 14D20, 32G34, 34G20, 58F05.

Key words: Stable parabolic connections, Representation of fundamental groups, Riemann-Hilbert correspondences, Symplectic structure, Painlevé equations, Garnier equations.

Partly supported by Grant-in-Aid for Scientific Research (B-12440008), (B-12440043), (Houga-138740023), (Wakate-B-15740018) the Ministry of Education, Culture, Sports, Science and Technology and JSPS-NWO exchange program.

*Department of Mathematics, Faculty of Science, Kyoto University, Kyoto, 606-8502, Japan

e-mail: inaba@math.kyoto-u.ac.jp 8581, Japan

e-mail: iwasaki@math.kyushu-u.ac.jp 8501, Japan

e-mail: mhsaito@math.kobe-u.ac.jp
}

** Faculty of Mathematics, Kyushu University, 6-10-1, Hakozaki, Higashi-ku, Fukuoka 812-

*** Department of Mathematics, Faculty of Science, Kobe University, Kobe, Rokko, 657-
\end{abstract}

(c) 2006 Research Institute for Mathematical Sciences, Kyoto University. All rights reserved. 
that the smooth parts of both moduli spaces have natural symplectic structures and $\mathbf{R H}$ is a symplectic resolution of singularities of $\mathcal{R}\left(\mathcal{P}_{n, \mathbf{t}}\right)_{\mathbf{a}}$, from which one can give geometric backgrounds for other interesting phenomena, like Hamiltonian structures, Bäcklund transformations, special solutions of these equations.

\section{$\S 1$. Introduction}

\section{$\S 1.1$ The purpose}

The purpose of the series of papers is to give a complete geometric background for Painlevé equations of type $V I$ or more generally for the so-called Garnier equations.

As is well-known, these nonlinear differential equations have the Painlevé property which means that generic solutions of these equations have no movable singularity except for poles so that solutions have the analytic continuations on whole of the universal covering of the space of time variables.

Besides the Painlevé property, there are several interesting phenomena related to these equations which have been investigated by many authors.

- Each of these equations can be written in a Hamiltonian system by a natural symplectic coordinate system ([Mal], [O3], [Iw1], [Iw2], [K], [ST]).

- These equations have natural parameters $\boldsymbol{\lambda}=\left(\lambda_{1}, \ldots, \lambda_{n}\right) \in \mathbf{C}^{n}$. Moreover there exist birational symmetries of these equations, called Bäcklund transformations of these equations, which act on both of variables and the parameters and preserve the equations. ([O4]).

- In Painlevé VI case, the group of all Bäcklund transformations is isomorphic to the affine Weyl group $W\left(D_{4}^{(1)}\right)$ of the type $D_{4}^{(1)}$. ([O4], [Sakai], [AL2], [NY], [IISO]).

- In Painlevé $V I$ case, if $\boldsymbol{\lambda} \in \mathbf{C}^{4}$ lies on a reflection hyperplane of a reflection in $W\left(D_{4}^{(1)}\right)$, then the corresponding equation has one parameter family of Riccati solutions. ([LY], [FA], [W], [STe], [SU]).

- A natural compactification of each space of initial conditions for $P_{V I}$, introduced by Okamoto [O1], can be obtained by a series of explicit blowing-ups of $\mathbf{P}_{\mathbf{C}}^{1} \times \mathbf{P}_{\mathbf{C}}^{1}$ or $\mathbf{F}_{2}$. The compactification is given by a smooth projective rational surface $S$ and it has a unique anti-canonical divisors $-K_{S}=Y$ such that $S \backslash Y_{\text {red }}$ is the space of initial conditions for $P_{V I}$. The pair $(S, Y)$ becomes an Okamoto-Painlevé pair of type $D_{4}^{(1)}$ in the sense of [STT]. (See also [Sakai]). 
Though these phenomena are discussed and investigated by many authors, the intrinsic mathematical background for these facts remains to be understood. Therefore, for example, it is worthwhile to ask the following fundamental questions:

- What is the geometric meaning of Painlevé property for these equations?

- What is the geometric meaning of the symplectic structure?

- What is the geometric origin of Bäcklund transformations?

- Why Riccati solutions or some classical solutions appear for the parameters on the reflection hyperplanes of the Bäcklund transformations?

In the series of the papers, the authors will give answers to these questions in a natural intrinsic framework.

\section{§1.2. Natural framework}

It is already known (cf. [F], [Ga], [Sch], [JMU], [O3], [Iw1] and [Iw2]) that these equations can be derived from the isomonodromic deformation of the systems of linear equations of rank 2 with regular singularities over $\mathbf{P}^{1}$ or equivalently linear connections on vector bundles of rank 2 with logarithmic poles over $\mathbf{P}^{1}$. Although we will follow this line in this paper, for several essential reasons, we have to introduce a slight generalization of linear connections which will be explained as follows.

Let $n \geq 3$ and let us set $T_{n}=\left\{\mathbf{t}=\left(t_{1}, \ldots, t_{n}\right) \in\left(\mathbf{P}_{\mathbf{C}}^{1}\right)^{n} \mid t_{i} \neq t_{j},(i \neq j)\right\}$, $\Lambda_{n}=\left\{\boldsymbol{\lambda}=\left(\lambda_{1}, \ldots, \lambda_{n}\right) \in \mathbf{C}^{n}\right\}$. Fix a data $(\mathbf{t}, \boldsymbol{\lambda}) \in T_{n} \times \Lambda_{n}$ and set $D(\mathbf{t})=$ $t_{1}+\cdots+t_{n}$. We also fix a line bundle $L$ on $\mathbf{P}_{\mathbf{C}}^{1}$ with a logarithmic connection $\nabla_{L}: L \longrightarrow L \otimes \Omega_{\mathbf{P}_{\mathbf{C}}^{1}}^{1}(D(\mathbf{t}))$.

A quadruple $\left(E, \nabla, \varphi, l=\left\{l_{i}\right\}_{i=1}^{n}\right)$ consisting of:

(1) a rank 2 vector bundle $E$ on $\mathbf{P}^{1}$,

(2) a logarithmic connection $\nabla: E \longrightarrow E \otimes \Omega_{\mathbf{P}^{1}}^{1}(D(\mathbf{t}))$

(3) a bundle isomorphism $\varphi: \wedge^{2} E \stackrel{\simeq}{\longrightarrow} L$ and

(4) one dimensional subspace $l_{i}$ of the fiber $E_{t_{i}}$ of $E$ at $t_{i}, l_{i} \subset E_{t_{i}}, i=1, \ldots, n$, is called a $(\mathbf{t}, \boldsymbol{\lambda})$-parabolic connection with the determinant $\left(L, \nabla_{L}\right)$ if they satisfy the following conditions: 
(1) for any local sections $s_{1}, s_{2}$ of $E$,

$$
(\varphi \otimes \mathrm{id})\left(\nabla s_{1} \wedge s_{2}+s_{1} \wedge \nabla s_{2}\right)=\nabla_{L}\left(\varphi\left(s_{1} \wedge s_{2}\right)\right)
$$

(2) $l_{i} \subset \operatorname{Ker}\left(\operatorname{res}_{t_{i}}(\nabla)-\lambda_{i}\right)$, that is, $\lambda_{i}$ is an eigenvalue of the residue $\operatorname{res}_{t_{i}}(\nabla)$ of $\nabla$ at $t_{i}$ and $l_{i}$ is a corresponding one-dimensional eigensubspace of $\operatorname{res}_{t_{i}}(\nabla)$.

We introduce a series of rational numbers $\boldsymbol{\alpha}=\left(\alpha_{1}, \ldots, \alpha_{2 n}\right)$ such that $0 \leq \alpha_{1}<\ldots<\alpha_{2 n}<1$, which is called a weight. By using a weight $\boldsymbol{\alpha}$, one can define parabolic degrees for $(\mathbf{t}, \boldsymbol{\lambda})$-parabolic connections $(E, \nabla, \varphi, l)$ and introduce the notion of the parabolic stability. Let $M_{n}^{\boldsymbol{\alpha}}(\mathbf{t}, \boldsymbol{\lambda}, L)$ be the coarse moduli space of stable $(\mathbf{t}, \boldsymbol{\lambda})$-parabolic connections on $\mathbf{P}^{1}$ with the determinant $\left(L, \nabla_{L}\right)$. Considering the relative setting over the parameter space $T_{n} \times \Lambda_{n}=$ $\{(\mathbf{t}, \boldsymbol{\lambda})\}$, we can construct a family of moduli spaces

$$
\pi_{n}: M_{n}^{\alpha}(L) \longrightarrow T_{n} \times \Lambda_{n}
$$

such that $\pi_{n}^{-1}(\mathbf{t}, \boldsymbol{\lambda}) \simeq M_{n}^{\alpha}(\mathbf{t}, \boldsymbol{\lambda}, L)$. Later, we have to extend the family by a finite étale covering $T_{n}^{\prime} \longrightarrow T_{n}$, and for simplicity, we denote it also by $\pi_{n}: M_{n}^{\alpha}(L) \longrightarrow T_{n}^{\prime} \times \Lambda_{n}$.

Next, let us fix $\mathbf{t} \in T_{n}$ and consider a representation $\rho: \pi_{1}\left(\mathbf{P}_{\mathbf{C}}^{1} \backslash D(\mathbf{t}), *\right)$ $\longrightarrow S L_{2}(\mathbf{C})$ of the fundamental group $\pi_{1}\left(\mathbf{P}_{\mathbf{C}}^{1} \backslash D(\mathbf{t}), *\right)$ with a fixed base point $* \in \mathbf{P}_{\mathbf{C}}^{1}$. Two representations $\rho_{1}$ and $\rho_{2}$ are said to be equivalent if there exists an element $P \in S L_{2}(\mathbf{C})$ such that $\rho_{2}=P^{-1} \rho_{1} P$. To each representation $\rho$, one can associate a local system $\mathbf{E}_{\rho}$ of rank 2 on $\mathbf{P}_{\mathbf{C}}^{1} \backslash D(\mathbf{t})$ with an isomorphism

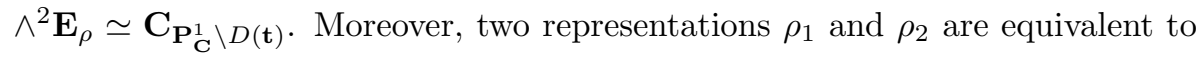
each other if and only if $\mathbf{E}_{\rho_{1}}$ and $\mathbf{E}_{\rho_{2}}$ are isomorphic as local systems. Hence the moduli space of the isomorphism classes of local systems on $\mathbf{P}^{1} \backslash D(\mathbf{t})$ with trivial determinants is isomorphic to the moduli space of equivalence classes of the representations.

Since $\pi_{1}\left(\mathbf{P}_{\mathbf{C}}^{1} \backslash D(\mathbf{t}), *\right)$ is a free group generated by $\gamma_{i}$ for $1 \leq i \leq n-1$ where $\gamma_{i}$ is a loop around the point $t_{i}$, such a representation can be determined by $M_{i}=\rho\left(\gamma_{i}\right) \in S L_{2}(\mathbf{C})$ for $1 \leq i \leq n-1$. Therefore the moduli space should be a quotient space of $S L_{2}(\mathbf{C})^{n-1}$ by a diagonal adjoint action of $S L_{2}(\mathbf{C})$.

However there is no canonical way to give a scheme structure on the set of equivalence classes of the representations. In this sense, we have to introduce a stronger equivalence relation. Two $S L_{2}(\mathbf{C})$-representations $\rho_{1}$ and $\rho_{2}$ of $\pi_{1}\left(\mathbf{P}_{\mathbf{C}}^{1} \backslash D(\mathbf{t}), *\right)$ are said to be Jordan equivalent if their semisimplifications are equivalent. This means that if a local system $\mathbf{E}_{\rho}$ is an extension of rank one local systems $L_{1}$ and $L_{2}$ one can not distinguish the extension classes. As is 
shown by Simpson [Sim2], the set of the Jordan equivalence classes of the local systems or representations is equal to the set of closed points of the categorical quotient

$$
\mathcal{R}\left(\mathcal{P}_{n, \mathbf{t}}\right)=S L_{2}(\mathbf{C})^{n-1} / A d\left(S L_{2}(\mathbf{C})\right),
$$

of $S L_{2}(\mathbf{C})^{n-1}$ by the diagonal adjoint action of $S L_{2}(\mathbf{C})$. The categorical quotients is defined as the affine scheme of the ring of invariant functions on $S L_{2}(\mathbf{C})^{n-1}$ by the action of $S L_{2}(\mathbf{C})$. (Cf. $\left.\S 4\right)$.

Fixing the canonical generators $\gamma_{i}(1 \leq i \leq n)$ of $\pi_{1}\left(\mathbf{P}_{\mathbf{C}}^{1} \backslash D(\mathbf{t}), *\right)$, to each representation $\rho: \pi_{1}\left(\mathbf{P}_{\mathbf{C}}^{1} \backslash D(\mathbf{t}), *\right) \longrightarrow S L_{2}(\mathbf{C})$, we can associate $n$-algebraic functions on $S L_{2}(\mathbf{C})^{n-1}$

$$
\operatorname{Tr}\left(\rho\left(\gamma_{i}\right)\right)=a_{i}, \quad \operatorname{Tr}\left(\rho\left(\left(\gamma_{1} \cdots \gamma_{n-1}\right)^{-1}\right)\right)=\operatorname{Tr}\left(\rho\left(\gamma_{n}\right)\right)=a_{n}
$$

which are clearly invariant under the adjoint action. Setting $\mathcal{A}_{n}=$ Spec $\mathbf{C}\left[a_{1}, \ldots, a_{n}\right] \simeq \mathbf{C}^{n}$, we obtain a natural morphism

$$
p_{n}: \mathcal{R}\left(\mathcal{P}_{n, \mathbf{t}}\right) \longrightarrow \mathcal{A}_{n}
$$

For a fixed closed point $\mathbf{a}=\left(a_{1}, \ldots, a_{n}\right) \in \mathcal{A}_{n}$, let us denote by $\mathcal{R}\left(\mathcal{P}_{n, \mathbf{t}}\right)_{\mathbf{a}}=$ $p_{n}^{-1}(\mathbf{a})$ the closed fiber at $\mathbf{a}$, that is, we set

$$
\mathcal{R}\left(\mathcal{P}_{n, \mathbf{t}}\right)_{\mathbf{a}}=\left\{[\rho] \in \mathcal{R}\left(\mathcal{P}_{n, \mathbf{t}}\right) \mid \operatorname{Tr}\left(\rho\left(\gamma_{i}\right)\right)=a_{i}, 1 \leq i \leq n\right\} .
$$

Moreover, taking a finite étale covering $T_{n}^{\prime} \longrightarrow T_{n}$ we can obtain a family of moduli spaces

$$
\phi_{n}: \mathcal{R}_{n} \longrightarrow T_{n}^{\prime} \times \mathcal{A}_{n}
$$

such that $\phi_{n}^{-1}(\mathbf{t}, \mathbf{a})=\mathcal{R}\left(\mathcal{P}_{n, \mathbf{t}}\right) \mathbf{a}($ cf. $\S 4)$.

Now, we have obtained two kinds of moduli spaces $M_{n}^{\boldsymbol{\alpha}}(\mathbf{t}, \boldsymbol{\lambda}, L)$ and $\mathcal{R}\left(\mathcal{P}_{n, \mathbf{t}}\right)_{\mathbf{a}}$ for fixed $(\mathbf{t}, \boldsymbol{\lambda}) \in T_{n}^{\prime} \times \Lambda_{n}$ and $(\mathbf{t}, \mathbf{a}) \in T_{n}^{\prime} \times \mathcal{A}_{n}$. Moreover we have two families of moduli spaces as in (1) and (2). (Note that we have already pulled back the family in (1) by the finite covering $T_{n}^{\prime} \longrightarrow T_{n}$.)

Next, let us assume that eigenvalues of $\operatorname{res}_{t_{i}}\left(\nabla_{L}\right)$ are integers for all $1 \leq i \leq$ $n$. Then we can define the Riemann-Hilbert correspondence $\mathbf{R H}_{n}: M_{n}^{\boldsymbol{\alpha}}(L) \longrightarrow$ $\mathcal{R}_{n}$ such that the following diagram commutes:

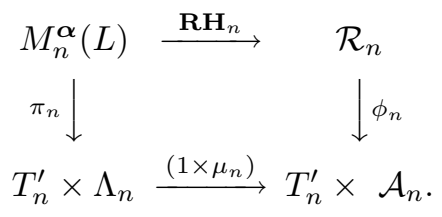


Here, the map $1 \times \mu_{n}$ in the bottom row in (3) is given by the map $(1 \times$ $\left.\mu_{n}\right)(\mathbf{t}, \boldsymbol{\lambda})=(\mathbf{t}, \mathbf{a})$ where

$$
a_{i}=2 \cos 2 \pi \lambda_{i} \quad \text { for } 1 \leq i \leq n .
$$

Under these relations, $\mathbf{R H}_{n}$ induces the analytic morphism of the fibers for each $(\mathbf{t}, \boldsymbol{\lambda}) \in T_{n}^{\prime} \times \Lambda_{n}$ :

$$
\mathbf{R H}_{\mathbf{t}, \boldsymbol{\lambda}}: M_{n}^{\boldsymbol{\alpha}}(\mathbf{t}, \boldsymbol{\lambda}, L) \longrightarrow \mathcal{R}\left(\mathcal{P}_{n, \mathbf{t}}\right)_{\mathbf{a}} .
$$

To define the correspondence, take a stable $(\mathbf{t}, \boldsymbol{\lambda})$-parabolic connection $(E, \nabla, \varphi$, $\left.\left\{l_{i}\right\}\right)$. Then restricting the connection $\nabla$ to $\mathbf{P}_{\mathbf{C}}^{1} \backslash D(\mathbf{t})$, define the local system on $\mathbf{P}_{\mathbf{C}}^{1} \backslash D(\mathbf{t})$ by

$$
\mathbf{E}(\nabla):=\operatorname{ker}\left(\nabla_{\mid \mathbf{P}_{\mathbf{C}}^{1} \backslash D(\mathbf{t})}\right)^{a n}
$$

(Here $\left(\nabla_{\mid \mathbf{P}_{\mathbf{C}}^{1} \backslash D(\mathbf{t})}\right)^{\text {an }}$ denotes the analytic connection associated to $\left.\nabla_{\mid \mathbf{P}_{\mathbf{C}}^{1} \backslash D(\mathbf{t})}\right)$. Then it is easy to see that the map $\left(E, \nabla, \varphi,\left\{l_{i}\right\}\right) \mapsto \mathbf{E}(\nabla)$ induces the correspondence in (3) or (5). Basically, our framework for understanding the Painlevé or Garnier equations is the Riemann-Hilbert correspondences in (3) and (5).

There exists one more thing which we should mention here. Let $\beta_{1}, \beta_{2}$ be positive integers, $\boldsymbol{\alpha}^{\prime}=\left(\alpha_{1}^{\prime}, \ldots, \alpha_{2 n}^{\prime}\right)$ a series of rational numbers with $0 \leq \alpha_{1}^{\prime}<$ $\ldots<\alpha_{2 n}^{\prime}<1$ and set $\boldsymbol{\beta}=\left(\beta_{1}, \beta_{2}\right)$. Setting $\boldsymbol{\alpha}=\boldsymbol{\alpha}^{\prime} \frac{\beta_{1}}{\beta_{1}+\beta_{2}}$, we obtain a weight $\boldsymbol{\alpha}$ for $(\mathbf{t}, \boldsymbol{\lambda})$-parabolic connections. (Note that since $\alpha_{2 n}=\alpha_{2 n}^{\prime} \frac{\beta_{1}}{\beta_{1}+\beta_{2}}<\frac{\beta_{1}}{\beta_{1}+\beta_{2}}$, this gives a restriction for the weight $\boldsymbol{\alpha}$ ). For the weight $\boldsymbol{\alpha}$, we consider the family of moduli spaces $M_{n}^{\alpha}(L) \longrightarrow T_{n}^{\prime} \times \Lambda_{n}$. On the other hand, we will introduce the notion of $\left(\boldsymbol{\alpha}^{\prime}, \boldsymbol{\beta}\right)$-stable $(\mathbf{t}, \boldsymbol{\lambda})$-parabolic $\phi$-connection which is a generalization of $\boldsymbol{\alpha}$-stable $(\mathbf{t}, \boldsymbol{\lambda})$-parabolic connections. The moduli space $\overline{M_{n}^{\boldsymbol{\alpha}^{\prime} \boldsymbol{\beta}}}(\mathbf{t}, \boldsymbol{\lambda}, L)$ contains the moduli space $M_{n}^{\boldsymbol{\alpha}}(\mathbf{t}, \boldsymbol{\lambda}, L)$ as a Zariski open set. Moreover we can construct the family of the moduli spaces such that the following diagram commutes:

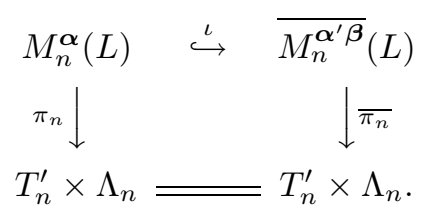




\section{$\S 1.3$. Main results}

In the framework as above, we can state our main results in this paper as follows.

1.3.1. Projectivity of the moduli space $\overline{M_{n}^{\alpha^{\prime} \boldsymbol{\beta}}}(\mathbf{t}, \boldsymbol{\lambda}, L)$, Smoothness, Irreducibility and the Symplectic Structure of $M_{n}^{\alpha}(\mathbf{t}, \boldsymbol{\lambda}, L)$

We first prove that the moduli space $\overline{M_{n}^{\boldsymbol{\alpha}^{\prime} \boldsymbol{\beta}}}(\mathbf{t}, \boldsymbol{\lambda}, L)$ is a projective scheme. Moreover one can show that the moduli space $M_{n}^{\boldsymbol{\alpha}}(\mathbf{t}, \boldsymbol{\lambda}, L)$ for each $(\mathbf{t}, \boldsymbol{\lambda}) \in$ $T_{n} \times \Lambda_{n}$ is smooth and endowed with a natural intrinsic symplectic structure induced by Serre duality of tangent complexes. The irreduciblity of the moduli space $M_{n}^{\boldsymbol{\alpha}}(\mathbf{t}, \boldsymbol{\lambda}, L)$ for each $(\mathbf{t}, \boldsymbol{\lambda}) \in T_{n} \times \Lambda_{n}$ follows from the irreduciblity of $\mathcal{R}\left(\mathcal{P}_{n, \mathbf{t}}\right)_{\mathbf{a}}$ via the Riemann-Hilbert correspondece (5).

Theorem 1.1 (Cf. Theorem 2.1, Theorem 5.2, Proposition 6.2 and Proposition 9.1).

(1) For a generic weight $\left(\boldsymbol{\alpha}^{\prime}, \boldsymbol{\beta}\right), \bar{\pi}_{n}: \overline{M_{n}^{\boldsymbol{\alpha}^{\prime} \boldsymbol{\beta}}}(L) \longrightarrow T_{n}^{\prime} \times \Lambda_{n}$ is a projective

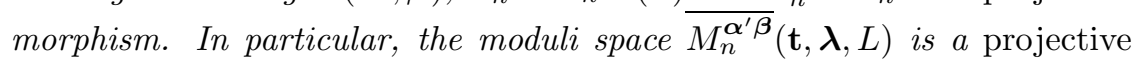
algebraic scheme for all $(\mathbf{t}, \boldsymbol{\lambda}) \in T_{n}^{\prime} \times \Lambda_{n}$.

(2) For a generic weight $\boldsymbol{\alpha}, \pi_{n}: M_{n}^{\boldsymbol{\alpha}}(L) \longrightarrow T_{n}^{\prime} \times \Lambda_{n}$ is a smooth morphism of relative dimension $2 n-6$ with irreducible closed fibers. Therefore, the moduli space $M_{n}^{\boldsymbol{\alpha}}(\mathbf{t}, \boldsymbol{\lambda}, L)$ is a smooth, irreducible algebraic variety of $d i$ mension $2 n-6$ for all $(\mathbf{t}, \boldsymbol{\lambda}) \in T_{n}^{\prime} \times \Lambda_{n}$.

Theorem 1.2 (Cf. Proposition 6.1). There exists a global relative 2form

$$
\Omega \in H^{0}\left(M_{n}^{\boldsymbol{\alpha}}(L), \Omega_{M_{n}^{\alpha}(L) / T_{n} \times \Lambda_{n}}^{2}\right)
$$

which induces a symplectic structure on each fiber of $\pi_{n}$. Consequently, for each $(\mathbf{t}, \boldsymbol{\lambda})$, the moduli space $M_{n}^{\boldsymbol{\alpha}}(\mathbf{t}, \boldsymbol{\lambda}, L)$ becomes a smooth symplectic algebraic variety.

\subsubsection{Irreducibility, symplectic structure and singularities of} $\mathcal{R}\left(\mathcal{P}_{n, \mathbf{t}}\right)_{\mathbf{a}}$

Let us call a data $\boldsymbol{\lambda} \in \Lambda_{n}$ a set of local exponents of connections.

\section{Definition 1.1.}

(1) A set of local exponents $\boldsymbol{\lambda}=\left(\lambda_{1}, \ldots, \lambda_{n}\right) \in \Lambda_{n}$ is said to be special if 
(a) $\boldsymbol{\lambda}$ is resonant, that is, for some $1 \leq i \leq n$,

$$
2 \lambda_{i} \in \mathbf{Z}
$$

(b) or $\boldsymbol{\lambda}$ is reducible, that is, for some $\left(\epsilon_{1}, \ldots, \epsilon_{n}\right) \in\{ \pm 1\}^{n}$

$$
\sum_{i=1}^{n} \epsilon_{i} \lambda_{i} \in \mathbf{Z}
$$

(2) If $\boldsymbol{\lambda} \in \Lambda_{n}$ is not special, $\boldsymbol{\lambda}$ is said to be generic.

(3) The data $\mathbf{a}=\left(a_{1}, \ldots, a_{n}\right) \in \mathcal{A}_{n}$ is said to be special if $\mu_{n}(\boldsymbol{\lambda})=\mathbf{a}$ for some special $\boldsymbol{\lambda} \in \Lambda_{n}$.

For a monodromy representation $\rho: \pi_{1}\left(\mathbf{P}^{1} \backslash D(\mathbf{t}), *\right) \longrightarrow S L_{2}(\mathbf{C})$, set $M_{i}=\rho\left(\gamma_{i}\right) \in S L_{2}(\mathbf{C})$ for $1 \leq i \leq n$. We consider the following conditions which are invariant under the adjoint action of $S L_{2}(\mathbf{C})$.

The representation $\rho$ is irreducible.

For all $i, 1 \leq i \leq n$, the local monodromy matrix $M_{i}$ around $t_{i}$ is not equal to $\pm I_{2}$.

Theorem 1.3 (Cf. Proposition 8.1, Proposition 6.3 and Theorem 7.1). Assume that $n \geq 4$.

(1) For any $\mathbf{a} \in \mathcal{A}_{n}$, the moduli space $\mathcal{R}\left(\mathcal{P}_{n, \mathbf{t}}\right)_{\mathbf{a}}$ is an irreducible affine scheme.

(2) Let $\mathcal{R}\left(\mathcal{P}_{n, \mathbf{t}}\right)_{\mathbf{a}}^{\sharp}$ be the Zariski dense open subset of $\mathcal{R}\left(\mathcal{P}_{n, \mathbf{t}}\right)_{\mathbf{a}}$ whose closed points satisfy the conditions (11) and (12). Then $\mathcal{R}\left(\mathcal{P}_{n, \mathbf{t}}\right)_{\mathbf{a}}^{\sharp}$ is smooth and there exists a natural symplectic form $\Omega_{1}$ on $\mathcal{R}\left(\mathcal{P}_{n, \mathbf{t}}\right)_{\mathbf{a}}^{\sharp}$.

(3) The codimension of the locus $\mathcal{R}\left(\mathcal{P}_{n, \mathbf{t}}\right)_{\mathbf{a}}^{\text {sing }}:=\mathcal{R}\left(\mathcal{P}_{n, \mathbf{t}}\right)_{\mathbf{a}} \backslash \mathcal{R}\left(\mathcal{P}_{n, \mathbf{t}}\right)_{\mathbf{a}}^{\sharp}$ is at least 2.

\subsubsection{Surjectivity and Properness of the Riemann-Hilbert corre- spondence}

Next, the most important result for the Riemann-Hilbert correspondence is the surjectivity and the properness. One can show that the correspondence $\mathbf{R H}_{\mathbf{t}, \boldsymbol{\lambda}}$ in (5) gives an analytic isomorphism between two moduli spaces if $\boldsymbol{\lambda} \in$ $\Lambda_{n}$ is generic (i.e. non-special). However, for a special $\boldsymbol{\lambda} \in \Lambda_{n}$, one can see that the map (5) contracts some subvarieties of $M_{n}^{\boldsymbol{\alpha}}(\mathbf{t}, \boldsymbol{\lambda}, L)$ to singular locus of $\mathcal{R}\left(\mathcal{P}_{n, \mathbf{t}}\right)_{\mathbf{a}}$. Note that since the correspondence is not an algebraic morphism, one can not directly apply the valuative criterion for the proof of the properness. 
Theorem 1.4 (Cf. Theorem 7.1). Under the notation above and assume that $n \geq 4$ and $\boldsymbol{\alpha}$ is general. For all $(\mathbf{t}, \boldsymbol{\lambda}) \in T_{n}^{\prime} \times \Lambda_{n}$, the Riemann-Hilbert correspondence

$$
\mathbf{R H}_{\mathbf{t}, \boldsymbol{\lambda}}: M_{n}^{\boldsymbol{\alpha}}(\mathbf{t}, \boldsymbol{\lambda}, L) \longrightarrow \mathcal{R}\left(\mathcal{P}_{n, \mathbf{t}}\right)_{\mathbf{a}}
$$

is a bimeromorphic proper surjective morphism.

\subsubsection{The Riemann-Hilbert correspondence as a symplectic reso- lution of singularities of $\mathcal{R}\left(\mathcal{P}_{n, \mathrm{t}}\right)_{\mathbf{a}}$}

Moreover, we can introduce the natural intrinsic symplectic structure on the smooth part $\mathcal{R}\left(\mathcal{P}_{n, \mathbf{t}}\right)_{\mathbf{a}}^{\sharp}$ of the moduli spaces $\mathcal{R}\left(\mathcal{P}_{n, \mathbf{t}}\right)_{\mathbf{a}}$. Together with the natural symplectic structure of the moduli space $M_{n}^{\boldsymbol{\alpha}}(\mathbf{t}, \boldsymbol{\lambda}, L)$, the map $\mathbf{R H}_{\mathbf{t}, \boldsymbol{\lambda}}$ gives a symplectic map, which means that the pullback of the symplectic structure on the smooth part of $\mathcal{R}\left(\mathcal{P}_{n, \mathbf{t}}\right)_{\mathbf{a}}$ coincides with the symplectic structure on $M_{n}^{\boldsymbol{\alpha}}(\mathbf{t}, \boldsymbol{\lambda}, L)$. This identification will be given by a kind of infinitesimal Riemann-Hilbert correspondence (cf. Lemma 6.6). Together with the surjectivity, the properness of $\mathbf{R H}_{\mathbf{t}, \boldsymbol{\lambda}}$ and the fact that $M_{n}^{\boldsymbol{\alpha}}(\mathbf{t}, \boldsymbol{\lambda}, L)$ is smooth, we can say that $\mathbf{R H}_{\mathbf{t}, \boldsymbol{\lambda}}$ gives an analytic symplectic resolution of the singularities of $\mathcal{R}\left(\mathcal{P}_{n, \mathbf{t}}\right)_{\mathbf{a}}$. Moreover, we can say that the map $\mathbf{R} \mathbf{H}_{n}$ in (3) gives a simultaneous resolution of the family $\phi_{n}: \mathcal{R}_{n} \longrightarrow T_{n}^{\prime} \times \mathcal{A}_{n}$ with the base extension $1 \times \mu_{n}: T_{n}^{\prime} \times \Lambda_{n} \longrightarrow T_{n}^{\prime} \times \mathcal{A}_{n}$. (For definition, see [Definition 4.26, [KM]]).

Theorem 1.5 (Theorem 7.1, Lemma 6.6). Under the assumption of Theorem (1.4), we have the following.

(1) For any $(\mathbf{t}, \boldsymbol{\lambda})$, let $\mathcal{R}\left(\mathcal{P}_{n, \mathbf{t}}\right)_{\mathbf{a}}^{\sharp}$ be as in Theorem 1.3 , and set $M_{n}^{\boldsymbol{\alpha}}(\mathbf{t}, \boldsymbol{\lambda}, L)^{\sharp}=$ $\mathbf{R H}_{\mathbf{t}, \boldsymbol{\lambda}}^{-1}\left(\mathcal{R}\left(\mathcal{P}_{n, \mathbf{t}}\right)_{\mathbf{a}}^{\sharp}\right)$. Then the Riemann-Hilbert correspondence gives an analytic isomorphism

$$
\mathbf{R H}_{\mathbf{t}, \boldsymbol{\lambda}, \mid M_{n}^{\alpha}(\mathbf{t}, \boldsymbol{\lambda}, L)^{\sharp}}: M_{n}^{\boldsymbol{\alpha}}(\mathbf{t}, \boldsymbol{\lambda}, L)^{\sharp} \stackrel{\simeq}{\longrightarrow} \mathcal{R}\left(\mathcal{P}_{n, \mathbf{t}}\right)_{\mathbf{a}}^{\sharp} .
$$

(Note that if $\boldsymbol{\lambda}$ is not special (cf. Definition 2. $(366(37)(3)), \mathcal{R}\left(\mathcal{P}_{n, \mathbf{t}}\right)_{\mathbf{a}}^{\sharp}=$ $\mathcal{R}\left(\mathcal{P}_{n, \mathbf{t}}\right)_{\mathbf{a}}$, hence $\mathbf{R H}_{\mathbf{t}, \boldsymbol{\lambda}}$ gives an analytic isomorphism between $M_{n}^{\boldsymbol{\alpha}}(\mathbf{t}, \boldsymbol{\lambda}, L)$ and $\left.\mathcal{R}\left(\mathcal{P}_{n, \mathbf{t}}\right)_{\mathbf{a}} \cdot\right)$

(2) The symplectic structures $\Omega$ restricted to $M_{n}^{\boldsymbol{\alpha}}(\mathbf{t}, \boldsymbol{\lambda}, L)^{\sharp}$ and $\Omega_{1}$ on $\mathcal{R}\left(\mathcal{P}_{n, \mathbf{t}}\right)_{\mathbf{a}}^{\sharp}$ can be identified with each other via $\mathbf{R H}_{\mathbf{t}, \boldsymbol{\lambda}}$, that is,

$$
\Omega_{\mid M_{n}^{\alpha}(\mathbf{t}, \boldsymbol{\lambda}, L)^{\sharp}}=\mathbf{R H}_{\mathbf{t}, \boldsymbol{\lambda} \mid M_{n}^{\alpha}(\mathbf{t}, \boldsymbol{\lambda}, L)^{\sharp}}^{*}\left(\Omega_{1}\right) \quad \text { on } \quad M_{n}^{\boldsymbol{\alpha}}(\mathbf{t}, \boldsymbol{\lambda}, L)^{\sharp} .
$$


(3) Putting together all results, the correspondence $\mathbf{R H}_{n}$ in (3) gives an analytic simultaneous symplectic resolution of singularities after the base extension $1 \times \mu_{n}: T_{n}^{\prime} \times \Lambda_{n} \rightarrow T_{n}^{\prime} \times \mathcal{A}_{n}$.

\section{§1.4. Painlevé and Garnier equations and their Painlevé property}

In the framework of this paper, we can derive the Painlevé and Garnier equations as follows. Take the universal covering map $\tilde{T}_{n} \longrightarrow T_{n}^{\prime} \longrightarrow T_{n}$ and pull back the diagram (3) to obtain the following commutative diagram:

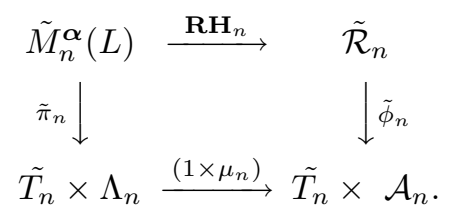

\subsubsection{The case of generic $\lambda$}

Now let us fix $\boldsymbol{\lambda} \in \Lambda_{n}$ and set $\mathbf{a}=\mu_{n}(\boldsymbol{\lambda})$. First, assume that $\boldsymbol{\lambda}$ is generic. We denote by $\left(\pi_{n}\right)_{\boldsymbol{\lambda}}: \tilde{M}_{n}^{\boldsymbol{\alpha}}(\boldsymbol{\lambda}, L) \longrightarrow \tilde{T}_{n}$ and $\left(\phi_{n}\right)_{\mathbf{a}}:\left(\tilde{\mathcal{R}}_{n}\right)_{\mathbf{a}} \longrightarrow \tilde{T}_{n}$ the families obtained by restricting the families in (16) to $\tilde{T}_{n} \times\{\boldsymbol{\lambda}\}$ and $\tilde{T}_{n} \times\{\mathbf{a}\}$. Moreover we denote by $\mathbf{R H}_{\boldsymbol{\lambda}}: \tilde{M}_{n}^{\boldsymbol{\alpha}}(\boldsymbol{\lambda}, L) \longrightarrow\left(\tilde{\mathcal{R}}_{n}\right)_{\mathbf{a}}$ the restriction of $\mathbf{R H}_{n}$ to the restricted families. Since $\boldsymbol{\lambda}$ is generic, $\mathbf{R H}_{\boldsymbol{\lambda}}$ induces an analytic isomorphism between $\tilde{M}_{n}^{\boldsymbol{\alpha}}(\boldsymbol{\lambda}, L)$ and $\left(\tilde{\mathcal{R}}_{n}\right)_{\mathbf{a}}$. Fix a point $\mathbf{t}_{0} \in T_{n}^{\prime}$. Since the original fibration $\left(\phi_{n}\right)_{\mathbf{a}}:\left(\mathcal{R}_{n}\right)_{\mathbf{a}} \longrightarrow T_{n}^{\prime} \times\{\mathbf{a}\}$ is locally trivial, we can obtain an isomorphism $\left(\tilde{\mathcal{R}}_{n}\right)_{\mathbf{a}} \simeq \mathcal{R}\left(\mathcal{P}_{n, \mathbf{t}_{0}}\right)_{\mathbf{a}} \times \tilde{T}_{n}$ and the following commutative diagram for fixed $\boldsymbol{\lambda}$ and $\mathbf{a}$.

$$
\begin{array}{ll}
\tilde{M}_{n}^{\boldsymbol{\alpha}}(\boldsymbol{\lambda}, L) \stackrel{\mathbf{R H}_{\boldsymbol{\lambda}}}{\simeq} & (\tilde{\mathcal{R}})_{\mathbf{a}} \simeq \mathcal{R}\left(\mathcal{P}_{n, \mathbf{t}_{0}}\right)_{\mathbf{a}} \times \tilde{T}_{n} \\
\left(\tilde{\pi}_{n}\right)_{\boldsymbol{\lambda}} \downarrow & \downarrow\left(\tilde{\phi}_{n}\right)_{\mathbf{a}} \\
\tilde{T}_{n} \times\{\boldsymbol{\lambda}\} \stackrel{=}{\longrightarrow} \tilde{T}_{n} \times\{\mathbf{a}\} .
\end{array}
$$

By using this global trivialization, for each closed point $\mathbf{x} \in \mathcal{R}\left(\mathcal{P}_{n, \mathbf{t}_{0}}\right)_{\mathbf{a}}$, we can define the unique constant section $s_{\mathbf{x}}: \tilde{T}_{n} \longrightarrow \mathcal{R}\left(\mathcal{P}_{n, \mathbf{t}_{0}}\right)_{\mathbf{a}} \times \tilde{T}_{n}$ for $\left(\phi_{n}\right)_{\mathbf{a}}$ by the formula $s_{\mathbf{x}}(\mathbf{t})=(\mathbf{x}, \mathbf{t})$. Pulling back this constant section $s_{\mathbf{x}}$ via $\mathbf{R H}_{\boldsymbol{\lambda}}$ we obtain the global analytic section $\tilde{s}_{\mathbf{x}}$ for the morphism $\left(\pi_{n}\right)_{\boldsymbol{\lambda}}$. Varying the initial points $\mathbf{x}$, we obtain the family of constant sections $\left\{s_{\mathbf{x}}\right\}_{\mathbf{x} \in \mathcal{R}\left(\mathcal{P}_{n, \mathbf{t}_{0}}\right)_{\mathbf{a}}}$ of $\mathcal{R}\left(\mathcal{P}_{n, \mathbf{t}_{0}}\right)_{\mathbf{a}} \times \tilde{T}_{n} \longrightarrow \tilde{T}_{n}$ and also the family of pullback sections $\left\{\tilde{s}_{\mathbf{x}}\right\}_{\mathbf{x} \in \mathcal{R}\left(\mathcal{P}_{n, \mathbf{t}_{0}}\right)_{\mathbf{a}}}$ for $\tilde{M}_{n}^{\boldsymbol{\alpha}}(\boldsymbol{\lambda}, L)$.

The family of sections $\left\{\tilde{s}_{\mathbf{x}}\right\}_{\mathbf{x} \in \mathcal{R}\left(\mathcal{P}_{n, \mathbf{t}_{0}}\right)_{\mathbf{a}}}$ gives the splitting homomorphism

$$
\tilde{v}_{\boldsymbol{\lambda}}:\left(\pi_{n}\right)_{\boldsymbol{\lambda}}^{*}\left(\Theta_{\tilde{T}_{n} \times\{\boldsymbol{\lambda}\}}\right) \longrightarrow \Theta_{\tilde{M}_{n}^{\alpha}(\boldsymbol{\lambda}, L)}
$$


for the natural surjective homomorphism $\Theta_{\tilde{M}_{n}^{\alpha}(\boldsymbol{\lambda}, L)} \longrightarrow\left(\pi_{n}\right)_{\boldsymbol{\lambda}}^{*}\left(\Theta_{\tilde{T}_{n} \times\{\boldsymbol{\lambda}\}}\right)$. Consider the following commutative diagram:

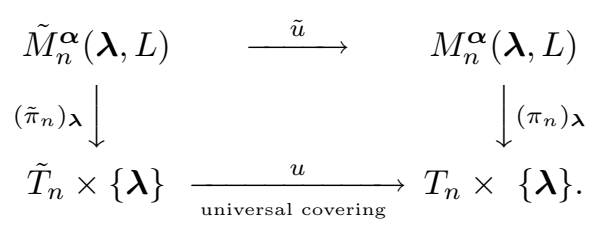

We can see that the splitting homomorphism (18) descends to a splitting homomorphism

$$
v_{\boldsymbol{\lambda}}:\left(\pi_{n}\right)_{\boldsymbol{\lambda}}^{*}\left(\Theta_{T_{n} \times\{\boldsymbol{\lambda}\}}\right) \longrightarrow \Theta_{M_{n}^{\alpha}(\boldsymbol{\lambda}, L)} .
$$

(One can show that this splitting is an algebraic homomorphism). Therefore, each algebraic vector field $\theta$ on $T_{n} \times\{\boldsymbol{\lambda}\}$ determines an algebraic vector field $v_{\boldsymbol{\lambda}}(\theta)$ on $M_{n}^{\boldsymbol{\alpha}}(\boldsymbol{\lambda}, L)$. The natural generators of the tangent sheaf of $T_{n} \times\{\boldsymbol{\lambda}\}$ can be given by

$$
\left\langle\frac{\partial}{\partial t_{1}}, \ldots, \frac{\partial}{\partial t_{n}}\right\rangle
$$

Defining

$$
v_{i}(\boldsymbol{\lambda})=v_{\boldsymbol{\lambda}}\left(\frac{\partial}{\partial t_{i}}\right) \in H^{0}\left(M_{n}^{\boldsymbol{\alpha}}(\boldsymbol{\lambda}, L), \Theta_{M_{n}^{\alpha}(\boldsymbol{\lambda}, L)}\right)
$$

we obtain the differential system

$$
\left\langle v_{1}(\boldsymbol{\lambda}), \ldots, v_{n}(\boldsymbol{\lambda})\right\rangle
$$

on $M_{n}^{\boldsymbol{\alpha}}(\boldsymbol{\lambda}, L)$. From the construction, it is obvious that these vector fields $\left\{v_{i}(\boldsymbol{\lambda})\right\}_{1 \leq i \leq n}$ commute to each other, that is, the differential systems are integrable. Since $\tilde{u}: \tilde{M}_{n}^{\boldsymbol{\alpha}}(\boldsymbol{\lambda}, L) \longrightarrow M_{n}^{\boldsymbol{\alpha}}(\boldsymbol{\lambda}, L)$ in (19) is also a covering map, each section $\tilde{s}_{\mathbf{x}}: \tilde{T}_{n} \times\{\boldsymbol{\lambda}\} \longrightarrow \tilde{M}_{n}^{\boldsymbol{\alpha}}(\boldsymbol{\lambda}, L)$ defines a multi-section for $M_{n}^{\boldsymbol{\alpha}}(\boldsymbol{\lambda}, L) \longrightarrow$ $T_{n} \times\{\boldsymbol{\lambda}\}$, which gives an integral submanifold of $M_{n}^{\boldsymbol{\alpha}}(\boldsymbol{\lambda}, L)$ for the differential system (22) at least locally. Hence the submanifold $\tilde{s}_{\mathbf{x}}\left(\tilde{T}_{n} \times\{\boldsymbol{\lambda}\}\right)$ of $\tilde{M}_{n}^{\boldsymbol{\alpha}}(\boldsymbol{\lambda}, L)$ given by the image of the section $\tilde{s}_{\mathbf{x}}$ can be considered as the integral submanifold (or a solution submanifold) for (22) over the universal covering space $\tilde{T}_{n}$. (It is natural to call the submanifold $\tilde{s}_{\mathbf{x}}\left(\tilde{T}_{n} \times\{\boldsymbol{\lambda}\}\right)$ an isomonodromic flow). Since the integral submanifold $\tilde{s}_{\mathbf{x}}\left(\tilde{T}_{n} \times\{\boldsymbol{\lambda}\}\right)$ is isomorphic to the parameter space $\tilde{T}_{n} \times\{\boldsymbol{\lambda}\}$ and the morphism $\tilde{\pi}_{n}: \tilde{M}_{n}^{\boldsymbol{\alpha}}(\boldsymbol{\lambda}, L) \longrightarrow \tilde{T}_{n} \times\{\boldsymbol{\lambda}\}$ is algebraic, we can conculde that

$$
\begin{aligned}
& \text { the diffrential system }\left\{v_{i}(\boldsymbol{\lambda})\right\}_{1 \leq i \leq n} \text { on } M_{n}^{\boldsymbol{\alpha}}(\boldsymbol{\lambda}, L) \text { has } \\
& \text { Painlevé property. (See Figure 1). }
\end{aligned}
$$




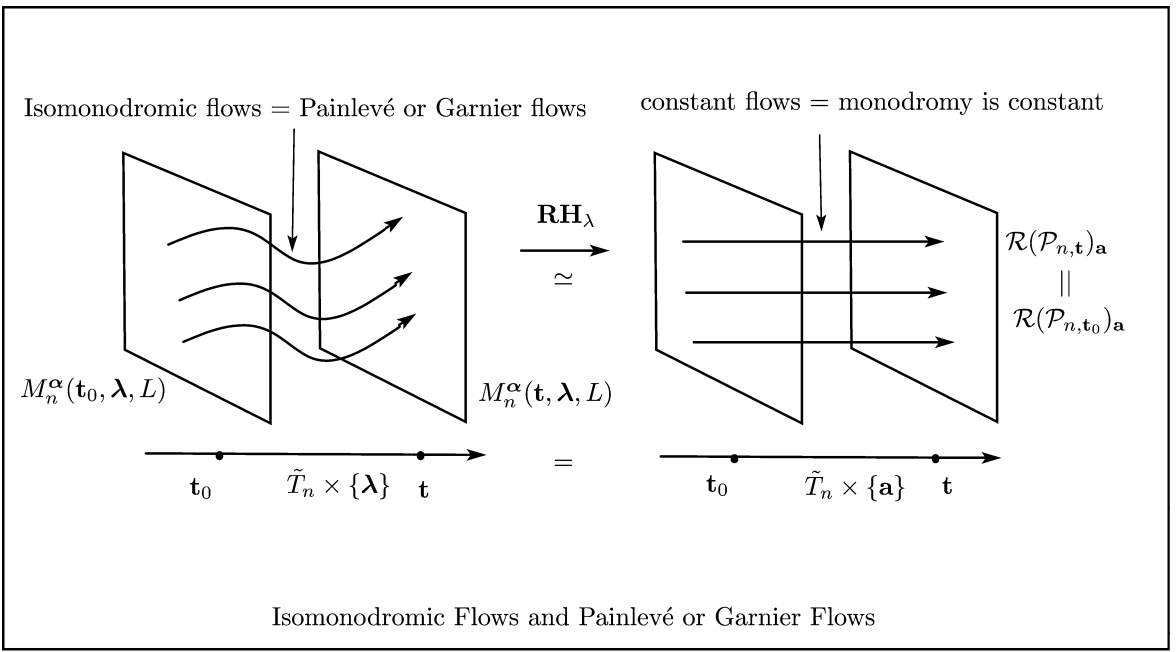

Figure 1. Riemann-Hilbert correspondence and isomonodromic flows for generic $\lambda$

Actually, the dynamical system on $M_{n}^{\boldsymbol{\alpha}}(\boldsymbol{\lambda}, L)$ detemined by $\left\{v_{i}(\boldsymbol{\lambda})\right\}_{1 \leq i \leq n}$ has geometric Painlevé property in the sense of [IISA] (cf. [Definition 2.2, [IISA]]). The differential system $\left\{v_{i}(\boldsymbol{\lambda})\right\}_{1 \leq i \leq n}$ in (22) is called Painlevé VI system for $n=4$ and Garnier system for $n \geq 5$. (Moreover we call each vector field $v_{i}(\boldsymbol{\lambda})$ Painlevé or Garnier vector field).

By using a suitable algebraic local coordinate system for $M_{n}^{\alpha}(\boldsymbol{\lambda}, L)$, one can write down the differential equations associated to $v_{i}(\boldsymbol{\lambda})$ and see that these differential systems are equivalent to known Painlevé $V I$ systems and Garnier systems. (It is possible to reduce the number of the time variables $t_{i}$ applying the automorphism of $\mathbf{P}_{\mathbf{C}}^{1}$ from $n$ to $\left.n-3\right)$. Moreover, one can apply a standard argument to show that the vector fields $v_{i}(\boldsymbol{\lambda})$ are algebraic vector fields on $M_{n}^{\alpha}(\boldsymbol{\lambda}, L)$.

\subsubsection{The case of special $\lambda$}

Next, let us consider the case when $\boldsymbol{\lambda}$ is special. We have the same commutative diagram as (17), however we encounter the following new phenomena.

(1) Although the moduli space $M_{n}^{\alpha}(\mathbf{t}, \boldsymbol{\lambda}, L)$ is nonsingular, the moduli space $\mathcal{R}\left(\mathcal{P}_{n, \mathbf{t}_{0}}\right)$ a has singularities.

(2) The Riemann-Hilbert correspondence $\mathbf{R H}_{\boldsymbol{\lambda}}: \tilde{M}_{n}^{\boldsymbol{\alpha}}(\boldsymbol{\lambda}, L) \longrightarrow\left(\tilde{\mathcal{R}}_{n}\right)_{\mathbf{a}}$ (or $\mathbf{R H}_{\mathbf{t}, \boldsymbol{\lambda}}: \tilde{M}_{n}^{\boldsymbol{\alpha}}(\mathbf{t}, \boldsymbol{\lambda}, L) \longrightarrow \mathcal{R}\left(\mathcal{P}_{n, \mathbf{t}_{0}}\right)_{\mathbf{a}}$ for a fixed $\left.\mathbf{t}\right)$ is still a bimeromorphic 
proper surjective map, but it contracts some families of compact subvarieties to singular locus of $\mathcal{R}\left(\mathcal{P}_{n, \mathbf{t}_{0}}\right)_{\mathbf{a}}$.

For example, in case when $n=4$ (Painlevé $V I$ case) and $\boldsymbol{\lambda}$ is special, $M_{n}^{\alpha}(\mathbf{t}, \boldsymbol{\lambda}, L)$ contains at least one (-2)-rational curve. For simplicity, assume that there is a unique (-2)-rational curve on $\tilde{M}_{4}^{\boldsymbol{\alpha}}\left(\mathbf{t}_{0}, \boldsymbol{\lambda}, L\right)$. Since $\mathcal{R}\left(\mathcal{P}_{n, \mathbf{t}_{0}}\right)$ a is an irreducible affine scheme, it cannot contain complete subvarieties of positive dimension, and hence $\mathbf{R H}_{\mathbf{t}_{0}, \boldsymbol{\lambda}}$ has to contract the (-2)-rational curve onto a singular point of type $A_{1}$. (See Figure 2). Let us define the subset $\tilde{M}_{n}^{\boldsymbol{\alpha}}(\boldsymbol{\lambda}, L)^{\sharp}$ the complement of the subvarieties contracted by $\mathbf{R H}_{\boldsymbol{\lambda}}$ in $\tilde{M}_{n}^{\boldsymbol{\alpha}}(\boldsymbol{\lambda}, L)$ and set $\left(\tilde{\mathcal{R}}_{n}\right)_{\mathbf{a}}^{\sharp}:=\mathbf{R H}_{\boldsymbol{\lambda}}\left(\tilde{M}_{n}^{\boldsymbol{\alpha}}(\boldsymbol{\lambda}, L)^{\sharp}\right)$ so that $\mathbf{R H}_{\boldsymbol{\lambda} \mid \tilde{M}_{n}^{\alpha}(\boldsymbol{\lambda}, L)^{\sharp}}: \tilde{M}_{n}^{\boldsymbol{\alpha}}(\boldsymbol{\lambda}, L)^{\sharp} \longrightarrow\left(\tilde{\mathcal{R}}_{n}\right)_{\mathbf{a}}^{\sharp}$ is an analytic isomorphism. For any $n \geq 4$, we can pull back the constant sections $s_{\mathbf{x}}$ by $\mathbf{R H}_{\boldsymbol{\lambda}}$ for $\mathbf{x} \in\left(\tilde{\mathcal{R}}_{n}\right)_{\mathbf{a}}^{\sharp}$ and obtain analytic sections $\tilde{s}_{\mathbf{x}}$, for $\left(\pi_{n}\right)_{\boldsymbol{\lambda}}$. Now consider the family $\left(\pi_{n}\right)_{\boldsymbol{\lambda}}: M_{n}^{\boldsymbol{\alpha}}(\boldsymbol{\lambda}, L) \longrightarrow T_{n} \times\{\boldsymbol{\lambda}\}$ over $T_{n} \times\{\boldsymbol{\lambda}\}$ and define $M_{n}^{\boldsymbol{\alpha}}(\boldsymbol{\lambda}, L)^{\sharp} \subset M_{n}^{\boldsymbol{\alpha}}(\boldsymbol{\lambda}, L)$ as above. Then we can also obtain mutually commutative Pailevé $V I$ or Garnier vector fields $v_{i}(\boldsymbol{\lambda})$ for $1 \leq i \leq n$ on $M_{n}^{\boldsymbol{\alpha}}(\boldsymbol{\lambda}, L)^{\sharp}$, and $\left\{v_{i}(\boldsymbol{\lambda})\right\}_{1 \leq i \leq n}$ defines an integrable differential system on $M_{n}^{\boldsymbol{\alpha}}(\boldsymbol{\lambda}, L)^{\sharp}$. Varying $\boldsymbol{\lambda}$, we obtain the set of algebraic vector fields $\left\{v_{i}\right\}_{1 \leq i \leq n}$ on $M_{n}^{\alpha}(L)^{\sharp}$ over $T_{n} \times \Lambda_{n}$. Since the codimension of $M_{n}^{\alpha}(L) \backslash M_{n}^{\alpha}(L)^{\sharp}$ in $M_{n}^{\alpha}(L)$ is greater than 2, one can extend the algebraic vector field $v_{i}$ to $M_{n}^{\boldsymbol{\alpha}}(L)$. Hence $v_{i}(\boldsymbol{\lambda})$ can also be extended to the total space of the family of the moduli spaces $\left(\pi_{n}\right)_{\boldsymbol{\lambda}}: M_{n}^{\boldsymbol{\alpha}}(\boldsymbol{\lambda}, L) \longrightarrow T_{n} \times\{\boldsymbol{\lambda}\}$. From the properness of the Riemann-Hilbert correspondence $\mathbf{R H}_{\boldsymbol{\lambda}}: \tilde{M}_{n}^{\boldsymbol{\alpha}}(\boldsymbol{\lambda}, L) \longrightarrow\left(\tilde{\mathcal{R}}_{n}\right)_{\mathbf{a}}$, we can conclude that the differential system $\left\{v_{i}(\boldsymbol{\lambda})\right\}_{1 \leq i \leq n}$ also has the geometric Painlevé property (cf. [IISA]).

The extended vector fields should be tangent to the family of contracted subvarieties (see Figure 2). The restriction of Painlevé $V I$ or Garnier vector fields $\left\{v_{i}(\boldsymbol{\lambda})\right\}_{1 \leq i \leq n}$ to the family of the contracted subvarieties yields integrable differential systems on the subvarieties whose solutions are given by a family of classical solutions like Riccati solutions for Painlevé VI system. For example, in the Painlevé $V I$ case, we can observe the following correspondence (cf. [STe], [IISA]). (See [Iw4] or [IISA] for the meaning of the nonlinear monodromy group for Painlevé $V I)$.

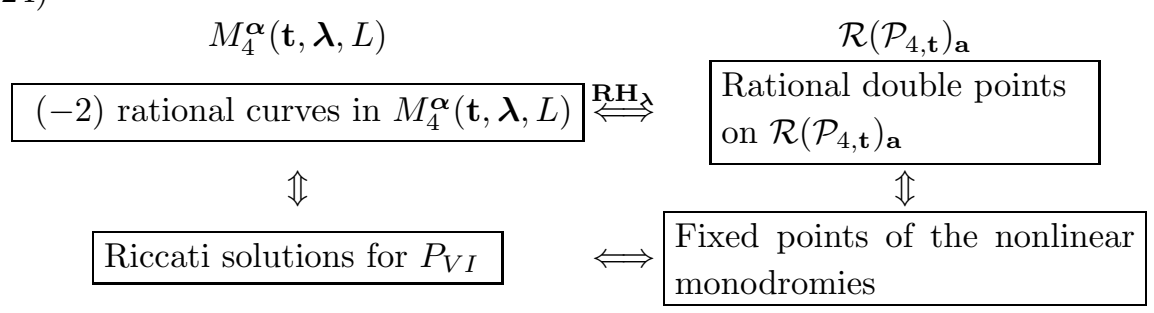



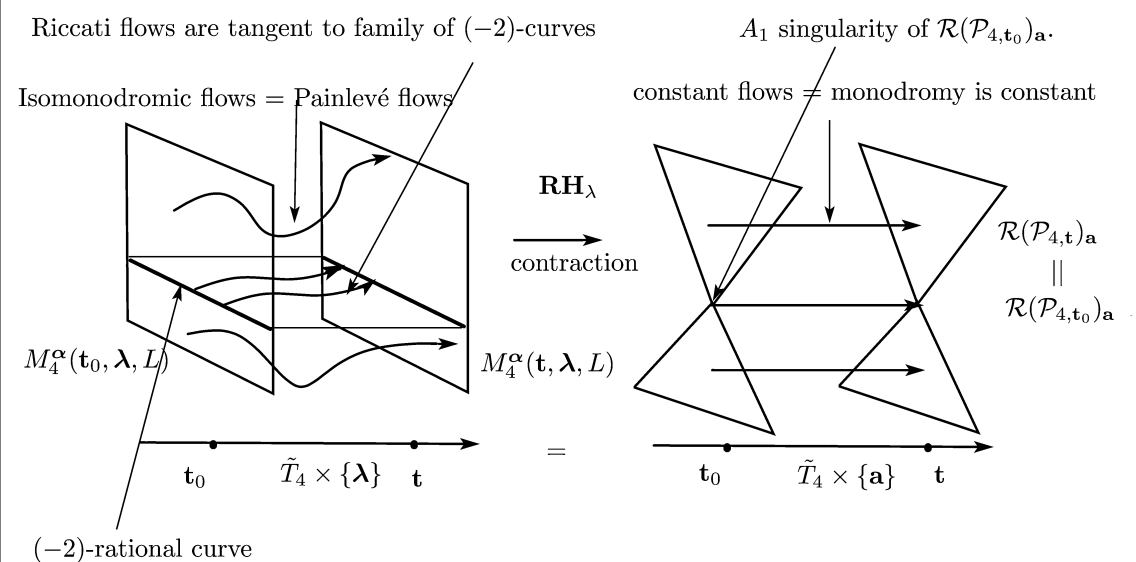

(-2)-rational curve

$\mathbf{R H}_{\boldsymbol{\lambda}}$ contracts (-2)-rational curves onto singular points of type $A_{1}$.

Figure 2. Riemann-Hilbert correspondence and isomonodromic flows for special $\lambda$

In Garnier case $(n \geq 5)$, when $\boldsymbol{\lambda}$ is reducible (10), one can obtain a special classical solution of the equation integrated by hypergeometric functions $F_{D}$ of Lauricella (cf. [Proposition $1.7[\mathrm{~K}]]$ ). One can see that these classical solutions of Garnier systems $\mathcal{G}_{n}$ correspond to the subvariety isomorphic to $\mathbf{P}^{n-3}$ which parametrizes reducible stable parabolic connections. Moreover when $\boldsymbol{\lambda}$ is resonant (9), the Garnier system $\mathcal{G}_{n}$ degenerates into a Riccati system over a Garnier system $\mathcal{G}_{n-1}$. A subvariety which can be contracted by $\mathbf{R H}_{\mathbf{t}, \boldsymbol{\lambda}}$ is isomorphic to $\mathbf{P}^{1}$-bundle over $M_{n-1}^{\boldsymbol{\alpha}}\left(\mathbf{t}^{\prime}, \boldsymbol{\lambda}^{\prime}, L^{\prime}\right)$ at a generic point of the contracted subvatiety.

\subsubsection{Painlevé $V I$ or Garnier equations parametrized by $\boldsymbol{\lambda} \in \Lambda_{n}$}

In the above formulation, for each fixed local exponent $\boldsymbol{\lambda} \in \Lambda_{n}$, we obtain the Painlevé or Garnier vector fields $v_{i}(\boldsymbol{\lambda})$ for $i, 1 \leq i \leq n$ as in (21) such that $\left\{v_{i}(\boldsymbol{\lambda})\right\}_{1 \leq i \leq n}$ forms an integrable differential system. Moreover the solution manifold for the differential system can be given by the isomonodromic flows. 
Varying the data $\boldsymbol{\lambda}$, we obtain vector fields

$$
v_{i} \in H^{0}\left(M_{n}^{\boldsymbol{\alpha}}(L), \Theta_{M_{n}^{\alpha}(L) / \Lambda_{n}}\right), 1 \leq i \leq n
$$

for $M_{n}^{\alpha}(L) \longrightarrow T_{n} \times \Lambda_{n}$ such that $v_{i \mid M_{n}^{\alpha}(\boldsymbol{\lambda}, L)}=v_{i}(\boldsymbol{\lambda})$.

\section{$\S 1.5$. The Hamiltonian system}

It is well-known that the Painlevé and Garnier equations can be written in the Hamiltonian systems. Now we can explain this as follows. Since the constant flows on $\left(\phi_{n}\right)_{\mathbf{a}}:\left(\tilde{\mathcal{R}}_{n}\right)_{\mathbf{a}} \longrightarrow \tilde{T}_{n}$ preserve the natural symplectic form $\Omega_{1}$ on the fiber $\mathcal{R}\left(\mathcal{P}_{n, \mathbf{t}}\right)_{\mathbf{a}}$ and the pullback of $\Omega_{1}$ by Riemann-Hilbert correspondence coincides with the symplectic structure $\Omega$ on $M_{n}^{\alpha}(\mathbf{t}, \boldsymbol{\lambda}, L)$, Painlevé or Garnier vector fields preserve the symplectic structure $\Omega$. Therefore, we can write the differential equations in the Hamiltonian systems by using suitable cannonical coordinate systems. Then an argument shows that such vector fields are actually regular algebraic, hence the Hamiltonians are given by regular algebraic functions.

\section{$\S 1.6$. The relation of the space of initial conditions of Okamoto or Okamoto-Painlevé pairs for $P_{V I}$}

In the case of $P_{V I}$, Okamoto [O1] constructed the spaces of initial conditions by blowing up the accessible singularities of 4 parameter family of Painlevé $V I$ equations. They are open algebraic surfaces which are complements of the anti-canonical divisors of projective rational surfaces obtained by the 8-point blowing-ups of $\mathbf{P}_{\mathbf{C}}^{1} \times \mathbf{P}_{\mathbf{C}}^{1}$ or $\mathbf{F}_{2}$. In [Sakai], [STT], the notion of the pairs of projective rational surfaces and its effective anti-canonical divisors with suitable conditions was introduced and its relation to Painlevé equation was revealed. In [STT], such a pair is called an Okamoto-Painlevé pair. Okamoto-Painlevé pairs of type $D_{4}^{(1)}$ correspond to Painlevé $V I$ equations. A semiuniversal family of Okamoto-Painlevé pairs is a family of projective surfaces $\pi: \overline{\mathcal{S}} \longrightarrow T_{4}^{\prime} \times \Lambda_{4}$ with the effective relative anticanonical divisor $\mathcal{Y}$ such that the configuration of the anticanonical divisor $\mathcal{Y}_{\mathbf{t}, \boldsymbol{\lambda}}$ is of type $D_{4}^{(1)}$. Then family of spaces of the initial conditions of Okamoto can be obtained as an open subset $\mathcal{S}:=\overline{\mathcal{S}} \backslash \mathcal{Y}$.

In the second part of this paper [IIS2], we will show that the family of Okamoto-Pailevé pairs $\overline{\mathcal{S}} \longrightarrow T_{4} \times \Lambda_{4}$ can be identified with the family of the moduli spaces $\overline{M_{4}^{\boldsymbol{\alpha}^{\prime} \boldsymbol{\beta}}}\left(\mathcal{O}_{\mathbf{P}^{1}}(-1)\right) \longrightarrow T_{4} \times \Lambda_{4}$, while $\mathcal{S} \longrightarrow T_{4} \times \Lambda_{4}$ can be identified with $M_{4}^{\alpha}\left(\mathcal{O}_{\mathbf{P}^{1}}(-1)\right) \longrightarrow T_{4} \times \Lambda_{4}$. (In this case, we will take 
$\beta_{1}=\beta_{2}=1$, hence $\boldsymbol{\alpha}=\boldsymbol{\alpha}^{\prime} \frac{\beta_{1}}{\beta_{1}+\beta_{2}}=\boldsymbol{\alpha}^{\prime} / 2$ ). So our constructions of the moduli spaces give an intrinsic meaning of Okamoto's explicit hard calculations in [O1].

\section{$\S 1.7$ The Bäcklund transformations-Symmetries of the equations}

In our framework, Bäcklund transformations for the Painlevé equations or Garnier equations can be defined as follows. Consider the Painlevé VI or Garnier system $\left\{v_{i}\right\}_{1 \leq i \leq n}$ defined in (25) and the family of moduli spaces $\pi_{n}: M_{n}^{\alpha}(L) \longrightarrow T_{n}^{\prime} \times \Lambda_{n}$.

Definition 1.2. The pair $(\tilde{s}, s)$ of a birational map $\tilde{s}: M_{n}^{\boldsymbol{\alpha}}(L) \cdots \rightarrow$ $M_{n}^{\boldsymbol{\alpha}}(L)\left(\right.$ or $\left.\tilde{s}: \overline{M_{n}^{\boldsymbol{\alpha}^{\prime} \boldsymbol{\beta}}}(L) \cdots \rightarrow \overline{M_{n}^{\boldsymbol{\alpha}^{\prime} \boldsymbol{\beta}}}(L)\right)$ and an affine transformation $s:$ $\Lambda_{n} \longrightarrow \Lambda_{n}$ is said to be a Bäcklund transformation of the differential system $\left\{v_{i}\right\}_{1 \leq i \leq n}$ or $\left\{v_{i}(\boldsymbol{\lambda})\right\}_{1 \leq i \leq n, \boldsymbol{\lambda} \in \Lambda_{n}}$ if they make the following diagram commutative:

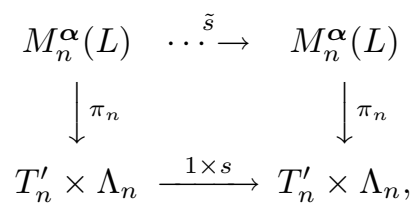

and it satisfies the condition:

$$
\tilde{s}_{*}\left(v_{i}\right)=v_{i} \text {, or equivalently } \tilde{s}_{*}\left(v_{i}(\boldsymbol{\lambda})\right)=v_{i}(s(\boldsymbol{\lambda})) .
$$

There exists a natural class of Bäcklund transformations of $M_{n}^{\alpha}(L)$ for any $n \geq 4$ which are induced by elementary transformations of stable parabolic connections (cf. §3). Since such transformations induce the identity on the moduli space of the monodromy representations via Riemann-Hilbert correspondence, we can conclude that the transformations preserve the vector field as in (27). (This notion is equivalent to the rational gauge transformation or Schlesinger transformation of connections). In $\S 3$, we will list up these kinds of Bäcklund transformations.

As for Painlevé VI equations, the group of the Bäcklund transformation in the above sense is isomorphic to the affine Weyl group $W\left(D_{4}^{(1)}\right)$ of type $D_{4}^{(1)}$, (cf. e.g. [O4], [IIS0]). The affine Weyl group $W\left(D_{4}^{(1)}\right)$ is generated by 5 reflections $s_{i}, i=0,1, \ldots, 4$ corresponding to the simple roots in the Dynkin diagram of type $D_{4}^{(1)}$ (see Figure 3 ). A natural faithful affine action of $W\left(D_{4}^{(1)}\right)$ 
to $\Lambda_{4}=\mathbf{C}^{4} \ni \boldsymbol{\lambda}=\left(\lambda_{j}\right)$ can be given by

$$
\begin{aligned}
& s_{i}\left(\lambda_{j}\right)=(-1)^{\delta_{i j}} \lambda_{j}, \quad i=1, \ldots, 4 \\
& s_{0}\left(\lambda_{i}\right)=\lambda_{i}-\frac{1}{2}\left(\sum_{k=1}^{4} \lambda_{k}\right)+\frac{1}{2} .
\end{aligned}
$$

Recalling the identification of the family $\overline{M_{4}^{\boldsymbol{\alpha}^{\prime} \boldsymbol{\beta}}}\left(\mathcal{O}_{\mathbf{P}^{1}}(-1)\right) \longrightarrow T_{4}^{\prime} \times \Lambda_{4}$ with the family of Okamoto-Painlevé pairs $\overline{\mathcal{S}} \longrightarrow T_{4}^{\prime} \times \Lambda_{4}$, one can see that the actions of $W\left(D_{4}^{(1)}\right)$ in (28) can be lifted to birational actions of the total space of the family $\bar{\pi}: \overline{\mathcal{S}} \longrightarrow T_{4}^{\prime} \times \Lambda$, that is, for each $s \in W\left(D_{4}^{(1)}\right)$, there exists a commutative diagram

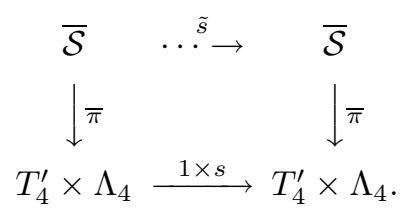

Moreover it is known [O4] that the actions preserve the Painlevé vector field $v_{i}$ in (25). That is, for each $s \in W\left(D_{4}^{(1)}\right)$, we have

$$
\tilde{s}_{*}\left(v_{i}(\boldsymbol{\lambda})\right)=v_{i}(s(\boldsymbol{\lambda})) \quad \text { for } \quad 1 \leq i \leq 4 .
$$

In our framework, it is easy to give an intrinsic reason why $\tilde{s_{i}}$ for $1 \leq i \leq 4$ preserve the vector field. It is simply because these come from elementary transformations. However, the origin of the transformation $\tilde{s_{0}}$ is still mysterious, and we cannot see any simple reason why $\tilde{s}_{0}$ preserves the vector field.

As some experts suggested to us, it may be plausible to believe that $\tilde{s}_{0}$ is induced by Laplace transformations of the stable connection. (The authors were informed by H. Sakai that M. Mazzocco gives some explanations for this fact on this line). For simplicity, let us call the Laplace transform of the original connection the dual of the connection. In general, the dual of logarithmic connections of rank 2 becomes a connection of higher rank which may not be logarithmic, so it is not so easy to identify the dual of the connection to the original one. Only in the case of $n=4$ (Painlevé case), we may miraculously identify the original connection with its dual or a further transformed object, so we have the extra Bäcklund transformation like $\tilde{s}_{0}$. It may be reasonable to consider the original connection and its dual at once. Then we may include the Laplace transformation as a part of the Bäcklund transformations.

After we have finished the first version of this paper, Philip Boalch informed us that he can obtain $\tilde{s}_{0}$ using the method of [Boa] as follows. One 
can embed a rank 2 connection with 4 -regular singular points over $\mathbf{P}^{1}$ into a rank 3 reducible connection. Then there is a simple operation for shifting the eigenvalues of the rank 3 connection. For a special value of shifting, one can obtain a rank 2 subconnection or a rank 1 subconnection in the shifted rank 3 connection, then take the rank 2 connection or the quotient of rank 1 subconnection. This gives a transformation from a rank 2 connection to another rank 2 connection whose transformation on $\boldsymbol{\lambda}$ gives $s_{0}$. Note that this transformation only works for the case of $n=4$. By using this result and another result in [Boa], he also gave a different proof of a result in [IISO].

Besides these stories, we should mention about the relation of the birational geometry and the Bäcklund transformations. As Saito and Umemura pointed out in [SU], Bäcklund transformations of Painlevé equations which are reflections with respect to roots of an affine root system are nothing but flops corresponding to $(-2)$-rational curves in Okamoto spaces of initial conditions.

From the definition of elementary transformations, we can easily see that the locus of indeterminacy of birational transformations correspond to the subvarieties which are contracted by the Riemann-Hilbert correspondence. Since the Riemann-Hilbert correspondence gives a simultaneous symplectic resolution of the singularities of the family $\phi_{n}: \mathcal{R}_{n} \longrightarrow T_{n}^{\prime} \times \mathcal{A}_{n}$, it is now obvious that those Bäcklund transformations are flops. (For definition and fundamental facts on flops, see $[\S 6,1[\mathrm{KM}]])$.

\subsubsection{Bäcklund transformations and the Riemann-Hilbert corre- spondence}

In [IIS0], we have proved that all of the Bäcklund transformations in $W\left(D_{4}^{(1)}\right)$ on $\overline{M_{4}^{\alpha}}(L) \longrightarrow T_{4}^{\prime} \times \Lambda_{4}$ induce essentially identity on the moduli space $\mathcal{R}_{4}$ after we take a finite quotient of $\mathcal{A}_{n}$. (Note that this is nontrivial only for $\left.\tilde{s_{0}}\right)$. Therefore in this sense, the group of the Bäcklund transformations $W\left(D_{4}^{(1)}\right)$ can be considered as the Galois group of the monodromy representations.

\section{$\S 1.8$. Related works}

It is worthwhile to discuss about some works related to this paper and to clarify what are really new in this paper.

The notion of $(\mathbf{t}, \boldsymbol{\lambda})$-parabolic connection on $\mathbf{P}^{1}$ is essentially introduced by Arinkin-Lysenko in [AL1] as a quasiparabolic $S L_{2}$-bundle. In [AL1], they also discussed about the moduli problem for quasiparabolic $S L_{2}$-bundles and consider the moduli space as an algebraic stack. In the case of $n=4$, under 


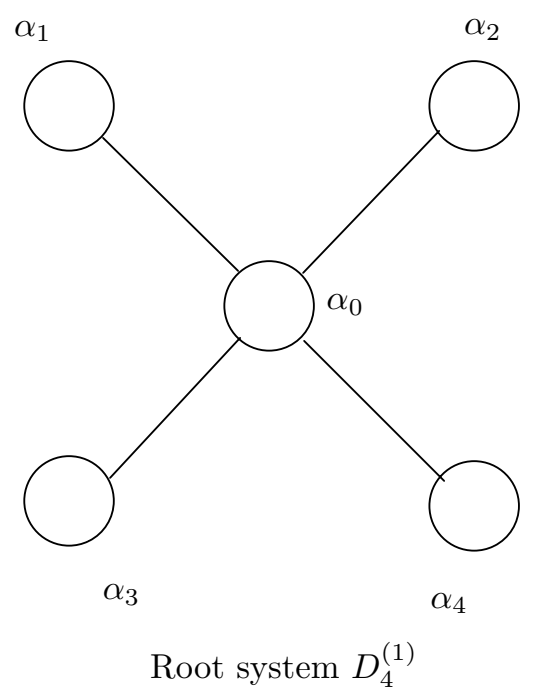

Figure 3. Dynkin diagram $D_{4}^{(1)}$

the assumption that $\boldsymbol{\lambda}$ is generic (cf. Definition 1.1), they give an explicit description of the coarse moduli space. Moreover, in [AL2], by using the explicit descriptions of the moduli spaces, they describe the group of automorphisms of the family of moduli spaces by using an explicit geometry of surfaces. Later, in $[\mathrm{A}]$, Arinkin introduced a notion of $\epsilon$-bundle, generalizing Deligne-Simpson's $\tau$-connections in [Sim1]. Again under the assumption that $\boldsymbol{\lambda}$ is generic Arinkin gives a compactification of the moduli space of quasiparabolic $S L_{2}$-bundles. Although the basic notions are introduced in their works, from the viewpoint of geometric background for Painlevé or Garnier equations, it is really necessary to construct the moduli spaces even for special $\boldsymbol{\lambda}$. For example, as we pointed out in 1.7 (cf. [SU]), some Bäcklund transformations of these equations are induced by flops in the terminology of the modern birational geometry and the center of flops are lying over the special parameter $\boldsymbol{\lambda}$.

In this sense, the advantage of introducing the notion of the stability for $(\mathbf{t}, \boldsymbol{\lambda})$-parabolic connection is obvious. In the GIT setting ([Mum]), despite considerable careful computations of stability, we can construct the fine moduli space of stable objects as smooth irreducible schemes even for special $\boldsymbol{\lambda}$. Moreover, we introduce the notion of parabolic $\phi$-connections which is a generalization of the notion of $\epsilon$-connections due to Arinkin-Deligne-Simpson and define the stability for them. One can understand the powers of these notions 
in Theorem 2.1 and Theorem 2.2.

The construction of the family of moduli spaces in (2) of $S L_{2}(\mathbf{C})$ monodromy representations are essentially due to Simpson [Sim2]. However a systematic treatment of nonlinear monodromies of the braid group is given by Dubrovin and Mazzocco [DM] for a special case of $n=4$, and by Iwasaki [Iw3], [Iw4] for the general case of $n=4$, and our construction of the family in this paper is taking care of the action of nonlinear monodromies of the braid groups to the moduli spaces.

Next, we would like to emphasize that only after we establish the natural setting in 1.2 it becomes possible to give a precise formulation of Hilbert's 21st problem for these cases. In our setting, the affirmative answer to the problem is equivalent to the surjectivity of the Riemann-Hilbert correspondences $\mathbf{R} \mathbf{H}_{n}$ and $\mathbf{R H}_{\mathbf{t}, \boldsymbol{\lambda}}$ in (3) and (5). As one can imagine easily, only reasonable result which one can apply to proof of the surjectivity is Deligne's theorem in [Del70].

Moreover the properness of $\mathbf{R H}_{\mathbf{t}, \boldsymbol{\lambda}}$ is also a new result. In the process of the proof, we need some analysis of the contraction induced by $\mathbf{R H}_{\mathbf{t}, \boldsymbol{\lambda}}$ and a technical lemma due to Professor A. Fujiki. The symplectic nature of the moduli spaces is discussed by many authors. (See for example [Go], [Iw1] [Iw2]). Iwasaki gave intrinsic symplectic structures on the moduli spaces of irreducible logarithmic connections on a nonsingular complete curve and show that they are obtained as the pullback of the symplectic structures on the moduli space of the irreducible representations.

Again, in this paper, we extended the symplectic structure to the whole moduli space of the stable parabolic connections and the smooth part of the moduli of the representations. Then one can show that these symplectic structures are identified via $\mathbf{R H}_{\mathbf{t}, \boldsymbol{\lambda}}$. Our proof here is based on some complexes of sheaves whose hypercohomologies describe the tangent spaces to the moduli spaces. Together with the surjectivity and the properness of $\mathbf{R H}_{\mathbf{t}, \boldsymbol{\lambda}}$ these results can be understood as $\mathbf{R H}_{\mathbf{t}, \boldsymbol{\lambda}}$ gives an analytic symplectic resolution of singularities in the sense of [Bea]. These kinds of viewpoints seem to be new, and this gives a clear explanation that a simple reflection in the group of Bäcklund transformations is nothing but a flop with respect to this resolution.

The derivation of Painlevé equations from the isomonodromic deformation of the linear connections is well-known. (See for example [JMU], [JM] and [O3]). However in most cases, one first takes a normalized linear connection written in certain coordinate systems and then writes up the Painlevé equations as the compatibility conditions for the extended linear connections. For a normalization, one has to assume that the vector bundle $E$ of rank 2 and 
degree 0 is isomorphic to $\mathcal{O}_{\mathbf{P}^{1}} \oplus \mathcal{O}_{\mathbf{P}^{1}}$, which is not true in general. In fact, the natural subscheme

$$
Z_{\mathbf{t}}=\left\{(E, \nabla, \varphi, l) \in \mathcal{M}_{n}^{\boldsymbol{\alpha}}(\mathbf{t}, \boldsymbol{\lambda}, L) ; E \not \varkappa \mathcal{O}_{\mathbf{P}^{1}} \oplus \mathcal{O}_{\mathbf{P}^{1}}\right\}
$$

of $\mathcal{M}_{n}^{\boldsymbol{\alpha}}(\mathbf{t}, \boldsymbol{\lambda}, L)$ is a non-empty divisor. We note that the isomonodromic flow starting from some point $p \in \mathcal{M}_{n}^{\boldsymbol{\alpha}}\left(\mathbf{t}_{0}, \boldsymbol{\lambda}, L\right) \backslash Z_{\mathbf{t}_{0}}$ does not stay inside the open subset $\bigcup_{\mathbf{t}}\left(\mathcal{M}_{n}^{\boldsymbol{\alpha}}(\mathbf{t}, \boldsymbol{\lambda}, L) \backslash Z_{\mathbf{t}}\right)$, that is, the flow intersects with $Z_{\mathbf{t}}$ for some $\mathbf{t}$. Therefore, in order to prove the Painlevé property of Painlevé VI or Garnier equations completely, we have to consider the whole space $\mathcal{M}_{n}^{\boldsymbol{\alpha}}(\mathbf{t}, \boldsymbol{\lambda}, L)$ and the properness of the Riemann-Hilbert correspondence is essential for our proof of Painlevé property. (For a discussion of various definitions of Painlevé property, see [IISA]. Moreover, for some proofs of analytic Painlevé property of isomonodromic deformations, see [Mal] and [Miw]). Moreover most former approaches avoid dealing with the case when $\boldsymbol{\lambda}$ is special, because one has to introduce the notion of the stability of the parabolic connections to obtain a good moduli space which is smooth and Hausdorff.

In our framework, we can also discuss the Painlevé or Garnier equations for special $\boldsymbol{\lambda}$ in a natural framework. Interestingly enough, the classical solutions for these equations can be derived from the family of subvarieties contracted by $\mathbf{R H}_{\mathbf{t}, \boldsymbol{\lambda}}$. Now, the geometric meaning of these facts becomes very clear. (For more detailed treatment in Painlevé $V I$ case, see [W], [STe] and [SU]).

We should mention that Nakajima [N] obtained a smooth moduli space of stable parabolic connections as the moduli space of filtered regular $D$-modules by the technique of the hyper-Kähler quotients of moment maps. Then he showed that the moduli space is diffeomorphic to the moduli space of parabolic Higgs bundles. Nitsure [Ni] also constructed the moduli space of the stable logarithmic connection without parabolic structures in GIT setting.

\section{Contents}

$\S 1 . \quad$ Introduction

$\S 1.1$. The purpose

§1.2. Natural framework

§1.3. Main results

§1.3.1. Projectivity of the moduli space $\overline{M_{n}^{\boldsymbol{\alpha}^{\prime} \boldsymbol{\beta}}}(\mathbf{t}, \boldsymbol{\lambda}, L)$, Smoothness, Irreducibility and the Symplectic Structure of $M_{n}^{\boldsymbol{\alpha}}(\mathbf{t}, \boldsymbol{\lambda}, L)$

§1.3.2. Irreducibility, symplectic structure and singularities of $\mathcal{R}\left(\mathcal{P}_{n, \mathbf{t}}\right)_{\mathbf{a}}$ 
$\S 1.3 .3$. Surjectivity and Properness of the Riemann-Hilbert correspondence

$\S 1.3 .4$. The Riemann-Hilbert correspondence as a symplectic resolution of singularities of $\mathcal{R}\left(\mathcal{P}_{n, \mathbf{t}}\right)_{\mathbf{a}}$

$\S 1.4$ Painlevé and Garnier equations and their Painlevé property

$\S 1.4 .1$. The case of generic $\boldsymbol{\lambda}$

$\S 1.4 .2$. The case of special $\boldsymbol{\lambda}$

§1.4.3. Painlevé $V I$ or Garnier equations parametrized by $\boldsymbol{\lambda} \in$ $\Lambda_{n}$

$\S 1.5$. The Hamiltonian system

$\S 1.6$. The relation of the space of initial conditions of Okamoto or Okamoto-Painlevé pairs for $P_{V I}$

$\S 1.7$. The Bäcklund transformations-Symmetries of the equations

§1.7.1. Bäcklund transformations and the Riemann-Hilbert correspondence

$\S 1.8$. Related works

§1.9. Acknowledgements

$\S 2$. Moduli Spaces of Stable Parabolic Connections on $\mathbf{P}^{1}$ and Their Compactifications

§2.1. Parabolic connections on $\mathbf{P}^{1}$

$\S 2.2$. The set of local exponents $\boldsymbol{\lambda} \in \Lambda_{n}$

$\S 2.3$. Parabolic degrees

$\S 2.4$. Stable parabolic $\phi$-connections

$\S 2.5$. The existence of moduli spaces and their properties

$\S 2.6$. The case of $n=4$ (Painlevé $V I$ case)

$\S 3$. Elementary Transformation of Parabolic Connections

$\S 3.1 . \quad$ Definition

§3.1.1. Definition of $E_{l m}^{+}$

§3.1.2. Definition of $E l m_{t_{i}}^{-}$

$\S 3.1 .3$. Tensoring a line bundle $L_{1}$

$\S 3.1 .4 . \quad R_{i}$ : Interchanging the eigenspaces

$\S 3.2$. Birational transformations arising from elementary transformations

§3.2.1. The group $B L_{n}$

$\S 4$. Moduli of Representations of Fundamental Groups

$\S 4.1$. The family of punctured projective lines and their fundamental groups

$\S 4.2$. The moduli space of $S L_{2}(\mathbf{C})$-representations 
$\S 4.3$. Construction of the family of moduli spaces $\phi_{n}: \mathcal{R}_{n} \longrightarrow T_{n}^{\prime} \times$ $\mathcal{A}_{n}$

§4.4. The case of $n=4$

$\S 5$. Construction of the Moduli Space $\overline{M_{n}^{\boldsymbol{\alpha}^{\prime} \boldsymbol{\beta}}}(\mathbf{t}, \boldsymbol{\lambda}, L)$ and Proof of Theorem

$2.1,(1)$

$\S 5.1$. Translation of the definition of parabolic $\phi$-connection

§5.2. Boundedness and Openness of stability

§5.3. Construction of the moduli space

$\S 5.4$. Projectivity of the moduli space

§5.5. Proof of Theorem 2.1 (1)

$\S 6$. Tangent Spaces of the Moduli Spaces and Canonical Symplectic Structure

§6.1. Tangent space to $M_{n}^{\boldsymbol{\alpha}}(\mathbf{t}, \boldsymbol{\lambda}, L)$

§6.2. The relative symplectic form $\Omega$ for $\pi_{n}: M_{n}^{\alpha} \longrightarrow T_{n}^{\prime} \times \Lambda_{n}$

§6.3. Smoothness of $M_{n}^{\boldsymbol{\alpha}}(\mathbf{t}, \boldsymbol{\lambda}, L)$ and its dimension

§6.4. Tangent space to $\mathcal{R}\left(\mathcal{P}_{n, \mathbf{t}}\right)_{\mathbf{a}}$

$\S 7 . \quad$ The Riemann-Hilbert Correspondence

§7.1. Definition of $\mathbf{R H}_{\mathbf{t}, \boldsymbol{\lambda}}$

\$7.2. Fundamental properties of Riemann-Hilbert correspondence

\$7.2.1. Proof of Theorem 7.1 except for the properness of $\mathbf{R H}_{\mathbf{t}, \boldsymbol{\lambda}}$

§7.3. The case of $n=4$

$\S 8$. Irreducibility of $\mathcal{R}\left(\mathcal{P}_{n, \mathbf{t}}\right)_{\mathbf{a}}$

$\S 9$. Irreducibility of $M_{n}^{\boldsymbol{\alpha}}(\mathbf{t}, \boldsymbol{\lambda}, L)$

$\S 10$. Properness of the Riemann-Hilbert Correspondence

$\S 11$. List of Notation

References

\section{§1.9. Acknowledgements}

First of all, the authors would like to thank Akira Fujiki who kindly informed them of the proof of Lemma 10.3. During the work, he has been kind enough to send several careful replies to our questions via emails. The authors are grateful to Hidetaka Sakai, Kazuo Okamoto and Kota Yoshioka for their valuable suggestions. The last author gives his sincere thanks to Eiichi Sato for his supports for travels from Kobe to Fukuoka, which made these 
fruitful joint works possible. The last author would like to thank JSPS-NWO exchange program for their financial support for visiting Utrecht University in September-October 2003. He also would like to thank Jan Stienstra for his interest to our work and his hospitality in Utrecht. Last but not least, it is pleasure of three authors to dedicate this paper to Kyoichi Takano on his 60th birthday with the great respect to his leadership on the studies of Painlevé equations as well as his friendships for younger generations.

\section{$\S 2 . \quad$ Moduli Spaces of Stable Parabolic Connections on $\mathbf{P}^{1}$ and Their Compactifications}

\section{§2.1. Parabolic connections on $\mathbf{P}^{1}$}

Let $n \geq 3$ and set

$$
\begin{gathered}
T_{n}=\left\{\left(t_{1}, \ldots, t_{n}\right) \in\left(\mathbf{P}^{1}\right)^{n} \quad \mid \quad t_{i} \neq t_{j},(i \neq j)\right\}, \\
\Lambda_{n}=\left\{\boldsymbol{\lambda}=\left(\lambda_{1}, \ldots, \lambda_{n}\right) \in \mathbf{C}^{n}\right\} .
\end{gathered}
$$

Fixing a data $(\mathbf{t}, \boldsymbol{\lambda})=\left(t_{1}, \ldots, t_{n}, \lambda_{1}, \ldots, \lambda_{n}\right) \in T_{n} \times \Lambda_{n}$, we define a reduced divisor on $\mathbf{P}^{1}$ as

$$
D(\mathbf{t})=t_{1}+\cdots+t_{n}
$$

Moreover we fix a line bundle $L$ on $\mathbf{P}^{1}$ with a logarithmic connection $\nabla_{L}$ : $L \longrightarrow L \otimes \Omega_{\mathbf{P}^{1}}^{1}(D(\mathbf{t}))$.

Definition 2.1. A (rank 2) $(\mathbf{t}, \boldsymbol{\lambda})$-parabolic connection on $\mathbf{P}^{1}$ with the determinant $\left(L, \nabla_{L}\right)$ is a quadruplet $\left(E, \nabla, \varphi,\left\{l_{i}\right\}_{1 \leq i \leq n}\right)$ which consists of

(1) a rank 2 vector bundle $E$ on $\mathbf{P}^{1}$,

(2) a logarithmic connection $\nabla: E \longrightarrow E \otimes \Omega_{\mathbf{P}^{1}}^{1}(D(\mathbf{t}))$

(3) a bundle isomorphism $\varphi: \wedge^{2} E \stackrel{\simeq}{\longrightarrow} L$

(4) one dimensional subspace $l_{i}$ of the fiber $E_{t_{i}}$ of $E$ at $t_{i}, l_{i} \subset E_{t_{i}}, i=1, \ldots, n$, such that

(a) for any local sections $s_{1}, s_{2}$ of $E$,

$$
\varphi \otimes i d\left(\nabla s_{1} \wedge s_{2}+s_{1} \wedge \nabla s_{2}\right)=\nabla_{L}\left(\varphi\left(s_{1} \wedge s_{2}\right)\right)
$$


(b) $l_{i} \subset \operatorname{Ker}\left(\operatorname{res}_{t_{i}}(\nabla)-\lambda_{i}\right)$, that is, $\lambda_{i}$ is an eigenvalue of the residue $\operatorname{res}_{t_{i}}(\nabla)$ of $\nabla$ at $t_{i}$ and $l_{i}$ is a one-dimensional eigensubspace of $\operatorname{res}_{t_{i}}(\nabla)$.

Definition 2.2. Two $(\mathbf{t}, \boldsymbol{\lambda})$-parabolic connections $\left(E_{1}, \nabla_{1}, \varphi\right.$, $\left.\left\{l_{i}\right\}_{1 \leq i \leq n}\right)\left(E_{2}, \nabla_{2}, \varphi^{\prime},\left\{l_{i}^{\prime}\right\}_{1 \leq i \leq n}\right)$ on $\mathbf{P}^{1}$ with the determinant $\left(L, \nabla_{L}\right)$ are isomorphic to each other if there is an isomorphism $\sigma: E_{1} \stackrel{\sim}{\longrightarrow} E_{2}$ and $c \in \mathbf{C}^{\times}$ such that the diagrams

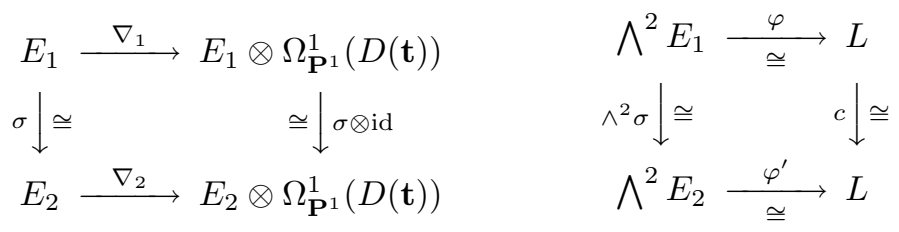

commute and $(\sigma)_{t_{i}}\left(l_{i}\right)=l_{i}^{\prime}$ for $i=1, \ldots, n$.

\section{§2.2. The set of local exponents $\lambda \in \Lambda_{n}$}

Note that a data $\boldsymbol{\lambda}=\left(\lambda_{1}, \ldots, \lambda_{n}\right) \in \Lambda_{n} \simeq \mathbf{C}^{n}$ specifies the set of eigenvalues of the residue matrix of a connection $\nabla$ at $\mathbf{t}=\left(t_{1}, \ldots, t_{n}\right)$, which will be called a set of local exponents of $\nabla$.

Definition 2.3. A set of local exponents $\boldsymbol{\lambda}=\left(\lambda_{1}, \ldots, \lambda_{n}\right) \in \Lambda_{n}$ is called special if

(1) $\boldsymbol{\lambda}$ is resonant, that is, for some $1 \leq i \leq n$,

$$
2 \lambda_{i} \in \mathbf{Z}
$$

(2) or $\boldsymbol{\lambda}$ is reducible, that is, for some $\left(\epsilon_{1}, \ldots, \epsilon_{n}\right) \in\{ \pm 1\}^{n}$

$$
\sum_{i=1}^{n} \epsilon_{i} \lambda_{i} \in \mathbf{Z} .
$$

If $\boldsymbol{\lambda} \in \Lambda_{n}$ is not special, $\boldsymbol{\lambda}$ is said to be generic.

Lemma 2.1. Let $\left(E, \nabla, \varphi, l=\left\{l_{i}\right\}\right)$ be a $(\mathbf{t}, \boldsymbol{\lambda})$-parabolic connection on $\mathbf{P}^{1}$ with the determinant $\left(L, \nabla_{L}\right)$. Assume that eigenvalues of $\operatorname{res}_{t_{i}}\left(\nabla_{L}\right)$ are integers for $1 \leq i \leq n$. Suppose that there exists a subline bundle $F \subset E$ such that $\nabla F \subset F \otimes \Omega_{\mathbf{P}^{1}}^{1}(D(\mathbf{t}))$. Then $\boldsymbol{\lambda}$ is reducible, that is, $\boldsymbol{\lambda}$ satisfies the condition (37). 
Proof (Cf. [Proposition 1.1, [AL1]]). Since we have a horizontal bundle isomorphism $\varphi: \wedge^{2} E \simeq L$ with respect to the connections, the eigenvalues of the residue matrix $\operatorname{res}_{t_{i}} \nabla$ at $t_{i}$ are given by $\lambda_{i}$ and $\operatorname{res}_{t_{i}}\left(\nabla_{L}\right)-\lambda_{i}$. Since $\nabla F \subset F \otimes \Omega_{\mathbf{P}^{1}}^{1}(D(\mathbf{t}))$, the subspace $F_{t_{i}} \subset E_{t_{i}}$ is an eigenspace of $\operatorname{res}_{t_{i}}(\nabla)$. Therefore the eigenvalue of $\operatorname{res}_{t_{i}}\left(\nabla_{\mid F}\right)$ is congruent to $\epsilon_{i} \lambda_{i}$ modulo $\mathbf{Z}$ for $\epsilon_{i}=1$ or -1 . The residue theorem says that

$$
\sum_{i=1}^{n} \operatorname{res}_{t_{i}}\left(\nabla_{\mid F}\right) \equiv-\operatorname{deg} F \equiv 0 \quad \bmod \quad \mathbf{Z}
$$

hence we have the lemma.

Remark 2.1. For $n=4$, the data $\boldsymbol{\lambda} \in \Lambda_{4}$ is special if and only if $\boldsymbol{\lambda} \in \Lambda_{4}$ lies on a reflection hyperplane of a reflection $s \in W\left(D_{4}^{(1)}\right)$.

\section{§2.3. Parabolic degrees}

Let us fix a series of positive rational numbers $\boldsymbol{\alpha}=\left(\alpha_{1}, \alpha_{2}, \ldots, \alpha_{2 n}\right)$, which is called a weight, such that

$$
0 \leq \alpha_{1}<\alpha_{2}<\cdots<\alpha_{i}<\cdots<\alpha_{2 n}<\alpha_{2 n+1}=1 .
$$

For a $(\mathbf{t}, \boldsymbol{\lambda})$-parabolic connection on $\mathbf{P}^{1}$ with the determinant $\left(L, \nabla_{L}\right)$, we can define the parabolic degree of $(E, \nabla, \varphi, l)$ with respect to the weight $\boldsymbol{\alpha}$ by

$$
\begin{aligned}
\operatorname{pardeg}_{\boldsymbol{\alpha}} E=\operatorname{pardeg}_{\boldsymbol{\alpha}}(E, \nabla, \varphi, l) & =\operatorname{deg} E+\sum_{i=1}^{n}\left(\alpha_{2 i-1} \operatorname{dim} E_{t_{i}} / l_{i}+\alpha_{2 i} \operatorname{dim} l_{i}\right) \\
& =\operatorname{deg} L+\sum_{i=1}^{n}\left(\alpha_{2 i-1}+\alpha_{2 i}\right) .
\end{aligned}
$$

Let $F \subset E$ be a rank 1 subbundle of $E$ such that $\nabla F \subset F \otimes \Omega_{\mathbf{P}^{1}}^{1}(D(\mathbf{t}))$. We define the parabolic degree of $\left(F, \nabla_{\mid F}\right)$ by

(40) $\operatorname{pardeg}_{\boldsymbol{\alpha}} F=\operatorname{deg} F+\sum_{i=1}^{n}\left(\alpha_{2 i-1} \operatorname{dim} F_{t_{i}} / l_{i} \cap F_{t_{i}}+\alpha_{2 i} \operatorname{dim} l_{i} \cap F_{t_{i}}\right)$

Definition 2.4. $\quad$ Fix a weight $\boldsymbol{\alpha}$. A $(\mathbf{t}, \boldsymbol{\lambda})$-parabolic connection $(E, \nabla, \varphi, l)$ on $\mathbf{P}^{1}$ with the determinant $\left(L, \nabla_{L}\right)$ is said to be $\boldsymbol{\alpha}$-stable (resp. $\boldsymbol{\alpha}$-semistable) if for every rank-1 subbundle $F$ with $\nabla(F) \subset F \otimes \Omega_{\mathbf{P}^{1}}^{1}(D(\mathbf{t}))$

$$
\operatorname{pardeg}_{\boldsymbol{\alpha}} F<\frac{\operatorname{pardeg}_{\boldsymbol{\alpha}} E}{2}, \quad\left(\text { resp. } \operatorname{pardeg}_{\boldsymbol{\alpha}} F \leq \frac{\operatorname{pardeg}_{\boldsymbol{\alpha}} E}{2}\right) .
$$

(For simplicity, " $\boldsymbol{\alpha}$-stable" will be abbreviated to "stable"). 
We define the coarse moduli space by

$M_{n}^{\boldsymbol{\alpha}}(\mathbf{t}, \boldsymbol{\lambda}, L)=\left\{(E, \nabla, \varphi, l) ; \begin{array}{l}\text { an } \boldsymbol{\alpha} \text {-stable }(\mathbf{t}, \boldsymbol{\lambda}) \text {-parabolic connection } \\ \text { with the determinant }\left(L, \nabla_{L}\right)\end{array}\right\} /$ isom.

\section{$\S 2.4$. Stable parabolic $\phi$-connections}

If $n \geq 4$, the moduli space $M_{n}^{\boldsymbol{\alpha}}(\mathbf{t}, \boldsymbol{\lambda}, L)$ never becomes projective nor complete. In order to obtain a compactification of the moduli space $M_{n}^{\boldsymbol{\alpha}}(\mathbf{t}, \boldsymbol{\lambda}, L)$, we will introduce the notion of a stable parabolic $\phi$-connection, or equivalently, a stable parabolic $\Lambda$-triple. Again, let us fix $(\mathbf{t}, \boldsymbol{\lambda}) \in T_{n} \times \Lambda_{n}$ and a line bundle $L$ on $\mathbf{P}^{1}$ with a connection $\nabla_{L}: L \rightarrow L \otimes \Omega_{\mathbf{P}^{1}}^{1}(D(\mathbf{t}))$.

Definition 2.5. The data $\left(E_{1}, E_{2}, \phi, \nabla, \varphi,\left\{l_{i}\right\}_{i=1}^{n}\right)$ is said to be a $(\mathbf{t}, \boldsymbol{\lambda})$ parabolic $\phi$-connection of rank 2 with the determinant $\left(L, \nabla_{L}\right)$ if $E_{1}, E_{2}$ are rank 2 vector bundles on $\mathbf{P}^{1}$ with $\operatorname{deg} E_{1}=\operatorname{deg} L, \phi: E_{1} \rightarrow E_{2}, \nabla: E_{1} \rightarrow$ $E_{2} \otimes \Omega_{\mathbf{P}^{1}}^{1}(D(\mathbf{t}))$ are morphisms of sheaves, $\varphi: \bigwedge^{2} E_{2} \stackrel{\sim}{\longrightarrow} L$ is an isomorphism and $l_{i} \subset\left(E_{1}\right)_{t_{i}}$ are one dimensional subspaces for $i=1, \ldots, n$ such that

(1) $\phi(f a)=f \phi(a)$ and $\nabla(f a)=\phi(a) \otimes d f+f \nabla(a)$ for $f \in \mathcal{O}_{\mathbf{P}^{1}}, a \in E_{1}$,

(2) $(\varphi \otimes \mathrm{id})\left(\nabla\left(s_{1}\right) \wedge \phi\left(s_{2}\right)+\phi\left(s_{1}\right) \wedge \nabla\left(s_{2}\right)\right)=\nabla_{L}\left(\varphi\left(\phi\left(s_{1}\right) \wedge \phi\left(s_{2}\right)\right)\right)$ for $s_{1}, s_{2} \in E_{1}$ and

(3) $\left.\left(\operatorname{res}_{t_{i}}(\nabla)-\lambda_{i} \phi_{t_{i}}\right)\right|_{l_{i}}=0$ for $i=1, \ldots, n$.

Definition 2.6. Two $(\mathbf{t}, \boldsymbol{\lambda})$ parabolic $\phi$-connections $\left(E_{1}, E_{2}, \phi, \nabla, \varphi\right.$, $\left.\left\{l_{i}\right\}\right),\left(E_{1}^{\prime}, E_{2}^{\prime}, \phi^{\prime}, \nabla^{\prime}, \varphi^{\prime},\left\{l_{i}^{\prime}\right\}\right)$ are said to be isomorphic to each other if there are isomorphisms $\sigma_{1}: E_{1} \stackrel{\sim}{\longrightarrow} E_{1}^{\prime}, \sigma_{2}: E_{2} \stackrel{\sim}{\longrightarrow} E_{2}^{\prime}$ and $c \in \mathbf{C} \backslash\{0\}$ such that the diagrams

$$
\begin{aligned}
& E_{1} \stackrel{\phi}{\longrightarrow} E_{2} \quad E_{1} \stackrel{\nabla}{\longrightarrow} E_{2} \otimes \Omega_{\mathbf{P}^{1}}^{1}(D(\mathbf{t})) \quad \bigwedge^{2} E_{2} \stackrel{\varphi}{\cong} L
\end{aligned}
$$

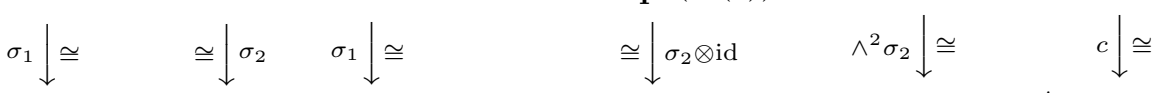

$$
\begin{aligned}
& E_{1}^{\prime} \stackrel{\phi^{\prime}}{\longrightarrow} E_{2}^{\prime} \quad E_{1}^{\prime} \stackrel{\nabla^{\prime}}{\longrightarrow} E_{2}^{\prime} \otimes \Omega_{\mathbf{P}^{1}}^{1}(D(\mathbf{t})) \quad \bigwedge^{2} E_{2}^{\prime} \stackrel{\varphi^{\prime}}{\cong} L
\end{aligned}
$$

commute and $\left(\sigma_{1}\right)_{t_{i}}\left(l_{i}\right)=l_{i}^{\prime}$ for $i=1, \ldots, n$.

Remark 2.2. Assume that two vector bundles $E_{1}, E_{2}$ and morphisms $\phi: E_{1} \rightarrow E_{2}, \nabla: E_{1} \rightarrow E_{2} \otimes \Omega_{\mathbf{P}^{1}}^{1}(D(\mathbf{t}))$ satisfying $\phi(f a)=f \phi(a), \nabla(f a)=$ $\phi(a) \otimes d f+f \nabla(a)$ for $f \in \mathcal{O}_{\mathbf{P}^{1}}, a \in E_{1}$ are given. If $\phi$ is an isomorphism, then $(\phi \otimes \mathrm{id})^{-1} \circ \nabla: E_{1} \rightarrow E_{1} \otimes \Omega_{\mathbf{P}^{1}}^{1}(D(\mathbf{t}))$ becomes a connection on $E_{1}$. 
Fix rational numbers $\alpha_{1}^{\prime}, \alpha_{2}^{\prime}, \ldots, \alpha_{2 n}^{\prime}, \alpha_{2 n+1}^{\prime}$ satisfying

$$
0 \leq \alpha_{1}^{\prime}<\alpha_{2}^{\prime}<\cdots<\alpha_{2 n}^{\prime}<\alpha_{2 n+1}^{\prime}=1
$$

and positive integers $\beta_{1}, \beta_{2}$. Setting $\boldsymbol{\alpha}^{\prime}=\left(\alpha_{1}^{\prime}, \ldots, \alpha_{2 n}^{\prime}\right), \boldsymbol{\beta}=\left(\beta_{1}, \beta_{2}\right)$, we obtain a weight $\left(\boldsymbol{\alpha}^{\prime}, \boldsymbol{\beta}\right)$ for parabolic $\phi$-connections.

Definition 2.7. Fix a sufficiently large integer $\gamma$. A parabolic $\phi$ connection $\left(E_{1}, E_{2}, \phi, \nabla, \varphi,\left\{l_{i}\right\}_{i=1}^{n}\right)$ is said to be $\left(\boldsymbol{\alpha}^{\prime}, \boldsymbol{\beta}\right)$-stable (resp. $\left(\boldsymbol{\alpha}^{\prime}, \boldsymbol{\beta}\right)$ semistable) if for any subbundles $F_{1} \subset E_{1}, F_{2} \subset E_{2}$ satisfying $\phi\left(F_{1}\right) \subset F_{2}$, $\nabla\left(F_{1}\right) \subset F_{2} \otimes \Omega_{\mathbf{P}^{1}}^{1}(D(\mathbf{t}))$ and $\left(F_{1}, F_{2}\right) \neq\left(E_{1}, E_{2}\right),(0,0)$, the inequality

$\frac{\beta_{1}\left(\operatorname{deg} F_{1}(-D(\mathbf{t}))\right)+\beta_{2}\left(\operatorname{deg} F_{2}-\gamma \operatorname{rank}\left(F_{2}\right)\right)+\sum_{i=1}^{n} \beta_{1}\left(\alpha_{2 i-1}^{\prime} d_{2 i-1}\left(F_{1}\right)+\alpha_{2 i}^{\prime} d_{2 i}\left(F_{1}\right)\right)}{\beta_{1} \operatorname{rank}\left(F_{1}\right)+\beta_{2} \operatorname{rank}\left(F_{2}\right)}$
$\quad<\leq \frac{\beta_{1}\left(\operatorname{deg} E_{1}(-D(\mathbf{t}))\right)+\beta_{2}\left(\operatorname{deg} E_{2}-\gamma \operatorname{rank}\left(E_{2}\right)\right)+\sum_{i=1}^{n} \beta_{1}\left(\alpha_{2 i-1}^{\prime} d_{2 i-1}\left(E_{1}\right)+\alpha_{2 i}^{\prime} d_{2 i}\left(E_{1}\right)\right)}{\beta_{1} \operatorname{rank}\left(E_{1}\right)+\beta_{2} \operatorname{rank}\left(E_{2}\right)}$

holds, where $d_{2 i-1}(F)=\operatorname{dim}\left(\left(F_{1}\right)_{t_{i}} / l_{i} \cap\left(F_{1}\right)_{t_{i}}\right), d_{2 i}\left(F_{1}\right)=\operatorname{dim}\left(\left(F_{1}\right)_{t_{i}} \cap l_{i}\right)$, $d_{2 i-1}\left(E_{1}\right)=\operatorname{dim}\left(\left(E_{1}\right)_{t_{i}} / l_{i}\right)(=1)$ and $d_{2 i}\left(E_{1}\right)=\operatorname{dim} l_{i}(=1)$.

Define the coarse moduli space by

(43) $\overline{M_{n}^{\boldsymbol{\alpha}^{\prime} \boldsymbol{\beta}}}(\mathbf{t}, \boldsymbol{\lambda}, L)$

$:=\left\{\left(E_{1}, E_{2}, \phi, \nabla, \varphi,\left\{l_{i}\right\}\right) ; \begin{array}{l}\text { a }\left(\boldsymbol{\alpha}^{\prime}, \boldsymbol{\beta}\right) \text {-stable }(\mathbf{t}, \boldsymbol{\lambda}) \text {-parabolic } \phi \text {-connection } \\ \text { with the determinant }\left(L, \nabla_{L}\right)\end{array}\right\} /$ isom.

For a given weight $\left(\boldsymbol{\alpha}^{\prime}, \boldsymbol{\beta}\right)$ and $1 \leq i \leq 2 n$, define a rational number $\alpha_{i}$ by

$$
\alpha_{i}=\frac{\beta_{1}}{\beta_{1}+\beta_{2}} \alpha_{i}^{\prime} .
$$

Then $\boldsymbol{\alpha}=\left(\alpha_{i}\right)$ satisfies the condition

$$
0 \leq \alpha_{1}<\alpha_{2}<\cdots<\alpha_{2 n}<\frac{\beta_{1}}{\left(\beta_{1}+\beta_{2}\right)}<1
$$

hence $\boldsymbol{\alpha}$ defines a weight for parabolic connections. It is easy to see that if we take $\gamma$ sufficiently large $\left(E, \nabla, \varphi,\left\{l_{i}\right\}\right)$ is $\boldsymbol{\alpha}$-stable if and only if the associated parabolic $\phi$-connection $\left(E, E, \operatorname{id}_{E}, \nabla, \varphi,\left\{l_{i}\right\}\right)$ is stable with respect to $\left(\boldsymbol{\alpha}^{\prime}, \boldsymbol{\beta}\right)$. Therefore we see that the natural map

$$
\left(E, \nabla, \varphi,\left\{l_{i}\right\}\right) \mapsto\left(E, E, \operatorname{id}_{E}, \nabla, \varphi,\left\{l_{i}\right\}\right)
$$


induces an injection

$$
M_{n}^{\boldsymbol{\alpha}}(\mathbf{t}, \boldsymbol{\lambda}, L) \hookrightarrow \overline{M_{n}^{\boldsymbol{\alpha}^{\prime} \boldsymbol{\beta}}}(\mathbf{t}, \boldsymbol{\lambda}, L)
$$

Conversely, assuming that $\boldsymbol{\beta}=\left(\beta_{1}, \beta_{2}\right)$ are given, for a weight $\boldsymbol{\alpha}=\left(\alpha_{i}\right)$ satisfying the condition (45), we can define $\alpha_{i}^{\prime}=\alpha_{i} \frac{\beta_{1}+\beta_{2}}{\beta_{1}}$ for $1 \leq i \leq 2 n$. Since $0 \leq \alpha_{1}^{\prime}<\alpha_{2}^{\prime}<\cdots<\alpha_{2 n}^{\prime}=\alpha_{2 n} \frac{\beta_{1}+\beta_{2}}{\beta_{1}}<1,\left(\boldsymbol{\alpha}^{\prime}, \boldsymbol{\beta}\right)$ give a weight for parabolic $\phi$-connections.

Moreover, considering the relative setting over $T_{n} \times \Lambda_{n}$, we can define two families of the moduli spaces

$$
\bar{\pi}_{n}: \overline{M_{n}^{\boldsymbol{\alpha}^{\prime} \boldsymbol{\beta}}}(L) \longrightarrow T_{n} \times \Lambda_{n}, \quad \pi_{n}: M_{n}^{\boldsymbol{\alpha}}(L) \longrightarrow T_{n} \times \Lambda_{n}
$$

such that the following diagram commutes;

$$
\begin{array}{ccc}
M_{n}^{\alpha}(L) & \stackrel{\iota}{\hookrightarrow} & \overline{M_{n}^{\alpha^{\prime} \boldsymbol{\beta}}}(L) \\
\pi_{n} \downarrow & & \\
T_{n} \times \Lambda_{n} \rightleftharpoons \bar{\pi}_{n} \\
& & T_{n} \times \Lambda_{n} .
\end{array}
$$

Here the fibers of $\pi_{n}$ and $\bar{\pi}_{n}$ over $(\mathbf{t}, \boldsymbol{\lambda}) \in T_{n} \times \Lambda_{n}$ are

$$
\pi_{n}^{-1}(\mathbf{t}, \boldsymbol{\lambda})=M^{\boldsymbol{\alpha}}(\mathbf{t}, \boldsymbol{\lambda}, L), \quad \bar{\pi}_{n}^{-1}(\mathbf{t}, \boldsymbol{\lambda})=\overline{M^{\boldsymbol{\alpha}^{\prime} \boldsymbol{\beta}}}(\mathbf{t}, \boldsymbol{\lambda}, L) .
$$

\section{$\S 2.5$. The existence of moduli spaces and their properties}

The following theorem is one of our fundamental results in this article which shows that the moduli spaces $\overline{M_{n}^{\boldsymbol{\alpha}^{\prime} \boldsymbol{\beta}}}(\mathbf{t}, \boldsymbol{\lambda}, L)$ and $M_{n}^{\boldsymbol{\alpha}}(\mathbf{t}, \boldsymbol{\lambda}, L)$ exist and they have good properties.

\section{Theorem 2.1.}

(1) Fix a weight $\boldsymbol{\beta}=\left(\beta_{1}, \beta_{2}\right)$. For a generic weight $\boldsymbol{\alpha}^{\prime}, \overline{\pi_{n}}: \overline{M_{n}^{\boldsymbol{\alpha}^{\prime} \boldsymbol{\beta}}}(L) \longrightarrow T_{n} \times$ $\Lambda_{n}$ is a projective morphism. In particular, the moduli space $\overline{M^{\boldsymbol{\alpha}^{\prime} \boldsymbol{\beta}}}(\mathbf{t}, \boldsymbol{\lambda}, L)$ is a projective algebraic scheme for all $(\mathbf{t}, \boldsymbol{\lambda}) \in T_{n} \times \Lambda_{n}$.

(2) For a generic weight $\boldsymbol{\alpha}, \pi_{n}: M_{n}^{\boldsymbol{\alpha}}(L) \longrightarrow T_{n} \times \Lambda_{n}$ is a smooth morphism of relative dimension $2 n-6$ with irreducible closed fibers. Therefore, the moduli space $M_{n}^{\boldsymbol{\alpha}}(\mathbf{t}, \boldsymbol{\lambda}, L)$ is a smooth, irreducible algebraic variety of $d i$ mension $2 n-6$ for all $(\mathbf{t}, \boldsymbol{\lambda}) \in T_{n} \times \Lambda_{n}$. 
The proof of Theorem 2.1 can be separated into 3 parts. The construction of the coarse moduli space of the parabolic $\phi$-connections over a projective smooth curve will be treated in Section 5. We deal with the relative settings and prove the projectivity of the morphism $\overline{\pi_{n}}: \overline{M_{n}^{\boldsymbol{\alpha}^{\prime} \boldsymbol{\beta}}}(L) \longrightarrow T_{n} \times \Lambda_{n}$. (Cf. Theorem 5.2). Since we have a natural embedding $M_{n}^{\boldsymbol{\alpha}}(L) \hookrightarrow \overline{M_{n}^{\boldsymbol{\alpha}^{\prime} \boldsymbol{\beta}}}(L)$, the existence of the moduli space $M_{n}^{\boldsymbol{\alpha}}(L)$ easily follows from the first assertion. The smoothness of the morphism $\pi_{n}: M_{n}^{\boldsymbol{\alpha}}(L) \longrightarrow T_{n} \times \Lambda_{n}$ follows from Proposition 6.2. Finally, the irreducibility of the moduli space $M_{n}^{\boldsymbol{\alpha}}(\mathbf{t}, \boldsymbol{\lambda}, L)$ is proved in Section 9, (cf. Proposition 9.1), based on the irreducibility of the moduli space $\mathcal{R}\left(\mathcal{P}_{n, \mathbf{t}}\right)$ a proved in Proposition 8.1.

\section{Remark 2.3.}

(1) As we mentioned in Introduction, we sometimes extend the base by an étale covering $T_{n}^{\prime} \longrightarrow T_{n}$ in Theorem 2.1, which causes no change in the proof.

(2) The structure of moduli spaces $M_{n}^{\boldsymbol{\alpha}}(L)$ and $\overline{M_{n}^{\boldsymbol{\alpha}^{\prime} \boldsymbol{\beta}}}(L)$ may depend on the weight $\boldsymbol{\alpha}$ and $\operatorname{deg} L$.

(3) The moduli space $M_{n}^{\alpha}(L)$ is a fine moduli space. In fact, we have the universal family over the moduli space $M_{n}^{\alpha}(L)$. See $\S 5$.

(4) When we describe the explicit algebraic or geometric structure of the moduli spaces $M_{n}^{\boldsymbol{\alpha}}(L)$ and $\overline{M_{n}^{\boldsymbol{\alpha}^{\prime} \boldsymbol{\beta}}}(L)$, it is convenient to fix a determinant line bundle $\left(L, \nabla_{L}\right)$. As a typical example of the determinant bundle is

$$
\left(L, \nabla_{L}\right)=\left(\mathcal{O}_{\mathbf{P}^{1}}\left(-t_{n}\right), d\right)
$$

where the connection is given by

$$
\nabla_{L}\left(z-t_{n}\right)=d\left(z-t_{n}\right)=\left(z-t_{n}\right) \otimes \frac{d z}{z-t_{n}}
$$

Here $z$ is an inhomogeneous coordinate of $\mathbf{P}^{1}=\operatorname{Spec} \mathbf{C}[z] \cup\{\infty\}$. For this $\left(L, \nabla_{L}\right)=\left(\mathcal{O}_{\mathbf{P}^{1}}\left(-t_{n}\right), d\right)$, we set

$M_{n}^{\boldsymbol{\alpha}}(\mathbf{t}, \boldsymbol{\lambda},-1)=M_{n}^{\boldsymbol{\alpha}}(\mathbf{t}, \boldsymbol{\lambda}, L), \quad\left(\operatorname{resp} . \overline{M_{n}^{\boldsymbol{\alpha}^{\prime} \boldsymbol{\beta}}}(\mathbf{t}, \boldsymbol{\lambda},-1)=\overline{M_{n}^{\boldsymbol{\alpha}^{\prime} \boldsymbol{\beta}}}(\mathbf{t}, \boldsymbol{\lambda}, L) \quad\right)$. 


\section{§2.6. The case of $n=4$ (Painlevé $V I$ case)}

We will deal with the case of $n=4$ which corresponds to Painlevé $V I$ equation. Let us fix a sufficiently large integer $\gamma$ and take a weight $\left(\boldsymbol{\alpha}^{\prime}, \boldsymbol{\beta}\right)$ for parabolic $\phi$-connections where $\boldsymbol{\alpha}^{\prime}=\left(\alpha_{1}^{\prime}, \ldots, \alpha_{8}^{\prime}\right), \boldsymbol{\beta}=\left(\beta_{1}, \beta_{2}\right), \gamma$ and fix $(\mathbf{t}, \boldsymbol{\lambda})=\left(t_{1}, \ldots, t_{4}, \lambda_{1}, \ldots, \lambda_{4}\right) \in T_{4} \times \Lambda_{4}$.

Then the corresponding weight $\alpha=\left(\alpha_{1}, \ldots, \alpha_{8}\right)$ for parabolic connections can be given by

$$
\alpha_{i}=\alpha_{i}^{\prime} \frac{\beta_{1}}{\beta_{1}+\beta_{2}} \quad 1 \leq i \leq 8 .
$$

Later, for simplicity, we will assume that $\beta_{1}=\beta_{2}$, hence $\boldsymbol{\alpha}=\boldsymbol{\alpha}^{\prime} / 2$. We also assume $\left(L, \nabla_{l}\right)=\left(\mathcal{O}_{\mathbf{P}^{1}}\left(-t_{n}\right), d\right)$ and in this case, we set

$$
\overline{M_{4}^{\boldsymbol{\alpha}^{\prime}}}(\mathbf{t}, \boldsymbol{\lambda},-1)=\overline{M_{4}^{\boldsymbol{\alpha}^{\prime} \boldsymbol{\beta}}}(\mathbf{t}, \boldsymbol{\lambda}, L), \quad \overline{M_{4}^{\boldsymbol{\alpha}^{\prime}}}(-1)=\overline{M_{4}^{\boldsymbol{\alpha}^{\prime} \boldsymbol{\beta}}}(L) .
$$

By Theorem 2.1, we can obtain the commutative diagram:

$$
\begin{array}{ccc}
M_{4}^{\alpha}(-1) & \stackrel{\iota}{\hookrightarrow} & \overline{M_{4}^{\boldsymbol{\alpha}^{\prime}}}(-1) \\
\pi_{4} \downarrow & & \\
T_{4} \times \Lambda_{4} & & \\
& & T_{4} \times \Lambda_{4},
\end{array}
$$

such that $\pi_{4}^{-1}((\mathbf{t}, \boldsymbol{\lambda})) \simeq M_{4}^{\boldsymbol{\alpha}}(\mathbf{t}, \boldsymbol{\lambda},-1)$ and $\bar{\pi}_{4}^{-1}(\mathbf{t}, \boldsymbol{\lambda}) \simeq \overline{M_{4}^{\boldsymbol{\alpha}^{\prime}}}(\mathbf{t}, \boldsymbol{\lambda},-1)$. (Note that $\left.\boldsymbol{\alpha}=\boldsymbol{\alpha}^{\prime} / 2\right)$. From Theorem 2.1, we see that for a generic weight $\boldsymbol{\alpha}^{\prime}, \bar{\pi}_{4}$ is a projective morphism and $\pi_{4}$ is a smooth morphism of relative dimension 2. In Part II, [IIS2], we will give detailed descriptions of the moduli spaces $M_{4}^{\boldsymbol{\alpha}}(\mathbf{t}, \boldsymbol{\lambda},-1)$ and $\overline{M_{4}^{\boldsymbol{\alpha}^{\prime}}}(\mathbf{t}, \boldsymbol{\lambda},-1)$. The following theorem shows that our family of the moduli space $\overline{M_{4}^{\alpha^{\prime}}}(-1) \longrightarrow T_{4} \times \Lambda_{4}$ can be identified with the family of Okamoto-Painlevé pairs constructed by Okamoto [O1]. (See also [Sakai], $[\mathrm{STT}])$. Note also that Arinkin and Lysenko [AL1] give isomorphisms between their moduli spaces and Okamoto spaces for generic $\boldsymbol{\lambda}$.

Theorem 2.2 (Cf. [IIS2]).

(1) For a suitable choice of a weight $\boldsymbol{\alpha}^{\prime}$, the morphism

$$
\bar{\pi}_{4}: \overline{M_{4}^{\boldsymbol{\alpha}^{\prime}}}(-1) \longrightarrow T_{4} \times \Lambda_{4}
$$

is projective and smooth. Moreover for any $(\mathbf{t}, \boldsymbol{\lambda}) \in T_{4} \times \Lambda_{4}$ the fiber $\bar{\pi}_{4}^{-1}(\mathbf{t}, \boldsymbol{\lambda}):=\overline{M_{4}^{\boldsymbol{\alpha}^{\prime}}}(\mathbf{t}, \boldsymbol{\lambda},-1)$ is irreducible, hence a smooth projective surface. 
(2) Let $\mathcal{D}=\overline{M_{4}^{\boldsymbol{\alpha}^{\prime}}}(-1) \backslash M_{4}^{\boldsymbol{\alpha}}(-1)$ be the complement of $M_{4}^{\boldsymbol{\alpha}}(-1)$ in $\overline{M_{4}^{\boldsymbol{\alpha}^{\prime}}}(-1)$. (Note that $\left.\boldsymbol{\alpha}=\boldsymbol{\alpha}^{\prime} / 2\right)$. Then $\mathcal{D}$ is a flat reduced divisor over $T_{4} \times \Lambda_{4}$.

(3) For each $(\mathbf{t}, \boldsymbol{\lambda})$, set

$$
\bar{S}_{\mathbf{t}, \boldsymbol{\lambda}}:=\bar{\pi}_{4}^{-1}(\mathbf{t}, \boldsymbol{\lambda}):=\overline{M_{4}^{\boldsymbol{\alpha}^{\prime}}}(\mathbf{t}, \boldsymbol{\lambda},-1)
$$

Then $\bar{S}_{\mathbf{t}, \boldsymbol{\lambda}}$ is a smooth projective surface which can be obtained by blowingups at 8 points of the Hirzebruch surface $\mathbf{F}_{2}=\operatorname{Proj}\left(\mathcal{O}_{\mathbf{P}^{1}}(-2) \oplus \mathcal{O}_{\mathbf{P}^{1}}\right)$ of degree 2. The surface has a unique effective anti-canonical divisor $-K_{S_{\mathbf{t}, \boldsymbol{\lambda}}}=$ $\mathcal{Y}_{\mathbf{t}, \boldsymbol{\lambda}}$ whose support is $\mathcal{D}_{\mathbf{t}, \boldsymbol{\lambda}}$. Then the pair

$$
\left(\bar{S}_{\mathbf{t}, \boldsymbol{\lambda}}, \mathcal{Y}_{\mathbf{t}, \boldsymbol{\lambda}}\right)
$$

is an Okamoto-Painlevé pair of type $D_{4}^{(1)}$. That is, the anti-canonical divisor $\mathcal{Y}_{\mathbf{t}, \boldsymbol{\lambda}}$ consists of 5-nodal rational curves whose configuration is same as Kodaira-Néron degenerate elliptic curves of type $D_{4}^{(1)}$ (=Kodaira type $\left.I_{0}^{*}\right)$. Moreover we have $\left(M_{4}^{\boldsymbol{\alpha}}(-1)\right)_{\mathbf{t}, \boldsymbol{\lambda}}=\left(\overline{M_{4}^{\boldsymbol{\alpha}^{\prime}}}(-1)\right)_{\mathbf{t}, \boldsymbol{\lambda}} \backslash \mathcal{Y}_{\mathbf{t}, \boldsymbol{\lambda}}$.

\section{$\S 3 . \quad$ Elementary Transformation of Parabolic Connections}

In this section, we will give basic definitions and some calculations of elementary transformations of stable parabolic connections.

\section{§3.1. Definition}

Let us fix a line bundle $L$ with a connection $\nabla_{L}: L \longrightarrow L \otimes \Omega_{\mathbf{P}^{1}}^{1}(D(\mathbf{t}))$ and we set

$$
\mu_{i}=\operatorname{res}_{t_{i}}\left(\nabla_{L}\right) \quad \text { for } 1 \leq i \leq n
$$

The residue theorem implies that $\sum_{i=1}^{n} \mu_{i}=-\operatorname{deg} L \in \mathbf{Z}$.

For each $i, 1 \leq i \leq n$, we set $L\left(t_{i}\right)=L \otimes \mathcal{O}_{\mathbf{P}^{1}}\left(t_{i}\right), L\left(-t_{i}\right)=L \otimes \mathcal{O}_{\mathbf{P}^{1}}\left(-t_{i}\right)$ and so on. We will define two elementary transformations which induce morphisms of moduli spaces.

$$
\begin{aligned}
& \operatorname{Elm}_{t_{i}}^{+}: M_{n}^{\boldsymbol{\alpha}}(\mathbf{t}, \boldsymbol{\lambda}, L) \longrightarrow M_{n}^{\boldsymbol{\alpha}}\left(\mathbf{t}, \boldsymbol{\lambda}^{\prime}, L\left(t_{i}\right)\right) \\
& \operatorname{Elm}_{t_{i}}^{-}: M_{n}^{\boldsymbol{\alpha}}(\mathbf{t}, \boldsymbol{\lambda}, L) \longrightarrow M_{n}^{\boldsymbol{\alpha}}\left(\mathbf{t}, \boldsymbol{\lambda}^{\prime}, L\left(-t_{i}\right)\right)
\end{aligned}
$$

Let $\left(E, \nabla_{E}, \varphi,\left\{l_{j}\right\}_{1 \leq j \leq n}\right)$ be a $(\mathbf{t}, \boldsymbol{\lambda})$-parabolic connection on $\mathbf{P}^{1}$ with the determinant $\left(L, \nabla_{L}\right)$. Note that the eigenvalues of $\operatorname{res}_{t_{i}}(\nabla)$ are given by the 
following table.

$$
E:\left(\begin{array}{r|rrrr|r} 
& t_{1} & t_{2} \cdots & t_{n-1} & t_{n} & \wedge^{2} E \\
\hline l_{j}=l_{j}^{+} & \lambda_{1} & \lambda_{2} \cdots & \lambda_{n-1} & \lambda_{n} & L \\
E_{t_{j}} / l_{j} \simeq l_{j}^{-} & \mu_{1}-\lambda_{1} & \mu_{2}-\lambda_{2} \cdots & \mu_{n-1}-\lambda_{n-1} & \mu_{n}-\lambda_{n} &
\end{array}\right)
$$

\subsubsection{Definition of Elm ti $_{t_{i}}^{+}$}

Take a subsheaf $F_{i}$ as

$$
E\left(-t_{i}\right) \subset F_{i} \subset E \text { such that } l_{i}=F_{i} / E\left(-t_{i}\right) \subset E_{t_{i}} \quad \text { and } \quad l_{i}\left(t_{i}\right)=F\left(t_{i}\right) / E
$$

and define

$$
E_{i}^{+}=F_{i}\left(t_{i}\right)=\operatorname{Ker}\left[E\left(t_{i}\right) \longrightarrow E\left(t_{i}\right) / F\left(t_{i}\right)=E\left(t_{i}\right)_{t_{i}} / l_{i}\left(t_{i}\right)\right] .
$$

Since $l_{i}$ is an eigenspace of $\operatorname{res}_{t_{i}}\left(\nabla_{E}\right)$, it is easy to see that $\nabla_{E}$ induces a connection

$$
\nabla_{E_{i}^{+}}: E_{i}^{+} \longrightarrow E_{i}^{+} \otimes \Omega_{\mathbf{P}^{1}}^{1}(D(\mathbf{t}))
$$

and $\varphi: \wedge^{2} E \longrightarrow L$ induces a horizontal isomorphism $\varphi^{\prime}: \wedge^{2} E_{i}^{+} \longrightarrow L\left(t_{i}\right)$. Moreover, one can see that the subspace $l_{i}^{\prime}=E_{t_{i}} / l_{i} \subset\left(E_{i}^{+}\right)_{t_{i}}$ defines a new parabolic structure $\left\{l_{j}^{\prime}\right\}_{j=1}^{n}$ with $l_{j}^{\prime}=l_{j}$ for $j \neq i$. Now we define

$$
E l m_{t_{i}}^{+}(E)=\left(E_{i}^{+}, \nabla_{E_{i}^{+}}, \varphi^{\prime},\left\{l_{i}^{\prime}\right\}\right)
$$

which is called an upper elementary transformation of $E$ at $t_{i}$. Since $l_{i}^{\prime} \simeq$ $E_{t_{i}} / l_{i},\left(E_{i}^{+}\right)_{t_{i}} / l_{i}^{\prime} \simeq l_{i} \otimes \mathcal{O}\left(t_{i}\right)$, we see that $\left(\operatorname{res}_{t_{i}}(\nabla)\right)_{\mid l_{i}^{\prime}}=\mu_{i}-\lambda_{i}$, $\left(\operatorname{res}_{t_{i}}(\nabla)\right)_{\mid\left(E_{i}^{+}\right)_{t_{i}} / l_{i}^{\prime}}=-1+\lambda_{i}$. Therefore the eigenvalues of the residues of $\nabla_{E_{i}^{+}}$ on $\operatorname{Elm}_{t_{i}}^{+}(E)=E_{i}^{+}$and the determinant $\wedge^{2} E_{i}^{+}$are given as follows.

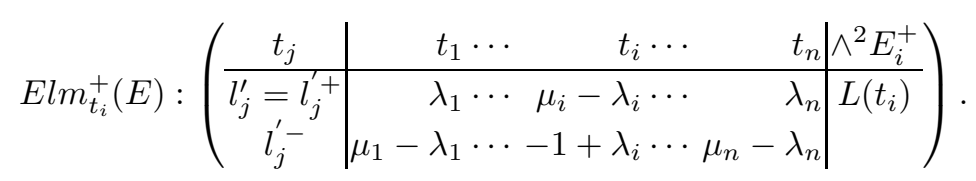

\subsubsection{Definition of $E l m_{t_{i}}^{-}$}

By using (59) subsheaf $F_{i} \subset E$ we also define a filtration of sheaves

$$
E_{i}^{-}=F_{i} \supset E\left(-t_{i}\right) \supset F_{i}\left(-t_{i}\right)
$$

which defines a parabolic connection $\left(E_{i}^{-}, \nabla_{E_{i}^{-}}, \varphi^{\prime}, l^{\prime}\right)$ such that

$$
l_{i}^{\prime}=E\left(-t_{i}\right) / F_{i}\left(-t_{i}\right)=\left(E_{t_{i}} / l_{i}\right) \otimes \mathcal{O}_{\mathbf{P}^{1}}\left(-t_{i}\right) .
$$


This is called a lower elementary transformation of $E$ at $t_{i}$ and will be denoted by

$$
\operatorname{Elm}_{t_{i}}^{-}(E):=\left(E_{i}^{-}, \nabla_{E_{i}^{-}}, \varphi^{\prime}, l^{\prime}\right) .
$$

Note that one has a horizontal isomorphism $\varphi^{\prime}: \wedge^{2} E_{i}^{-} \stackrel{\simeq}{\longrightarrow} L\left(-t_{i}\right)$ and the eigenvalues of the residues of $\nabla_{E_{i}^{-}}$on $\operatorname{Elm}_{t_{i}}^{-}(E)=E_{i}^{-}$are given as follows.

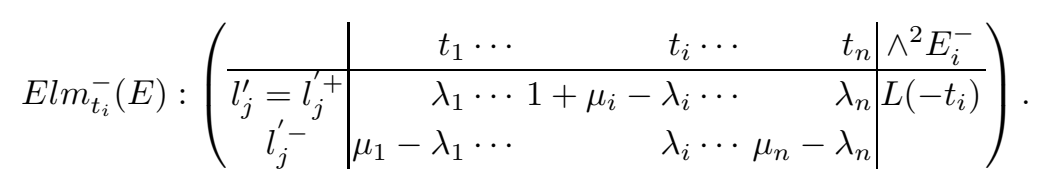

\subsubsection{Tensoring a line bundle $L_{1}$}

Let $L_{1}$ be a line bundle with a logarithmic connection $\nabla_{L_{1}}$ and set $\nu_{j}=$ $\operatorname{res}_{t_{j}}\left(\nabla_{L_{1}}\right)$ for $1 \leq j \leq n$. We can define a transformation $\otimes\left(L_{1}, \nabla_{L_{1}}\right)$ by

$$
\left(E, \nabla_{E}, \varphi,\left\{l_{j}\right\}\right) \mapsto\left(E \otimes L_{1}, \nabla_{E \otimes L_{1}}, \varphi^{\prime},\left\{l_{j} \otimes L_{1}\right\}\right)
$$

which induces a morphism of moduli spaces

$$
\otimes\left(L_{1}, \nabla_{L_{1}}\right): M_{n}^{\boldsymbol{\alpha}}(\mathbf{t}, \boldsymbol{\lambda}, L) \longrightarrow M_{n}^{\boldsymbol{\alpha}}\left(\mathbf{t}, \boldsymbol{\lambda}^{\prime}, L \otimes\left(L_{1}\right)^{\otimes 2}\right) .
$$

The set of eigenvalues of new connection can be given as follows.

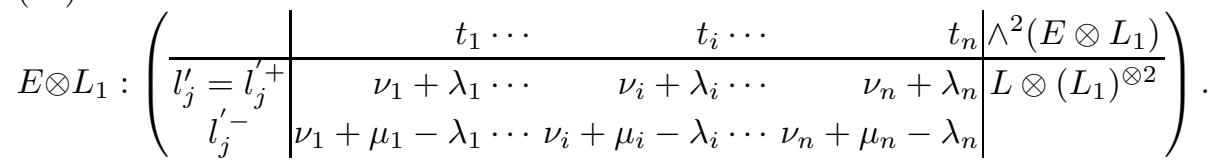

\subsection{4. $R_{i}$ : Interchanging the eigenspaces}

Under the assumption

$$
\lambda_{i} \neq \mu_{i}-\lambda_{i}
$$

we see that there are unique eigenspaces $l_{i}^{+}=l_{i}$ and $l_{i}^{-}$of $\operatorname{res}_{t_{i}}\left(\nabla_{E}\right)$ with the eigenvalues $\lambda_{i}$ and $\mu_{i}-\lambda_{i}$ respectively. Interchanging the eigenspaces $l_{i}^{+}$and $l_{i}^{-}$and keeping the other eigenspaces $l_{j} j \neq i$ unchanged, we obtain a new parabolic connection

$$
R_{i}(E)=\left(E, \nabla_{E}, \varphi,\left\{l_{i}^{\prime}\right\}\right) .
$$

If $\lambda_{i}=\mu_{i}-\lambda_{i}$, let us define $R_{i}(E)=\left(E, \nabla_{E}, \varphi,\left\{l_{i}\right\}\right)$, that is, $R_{i}=I d$. 
The set of eigenvalues of new connection can be given as follows.

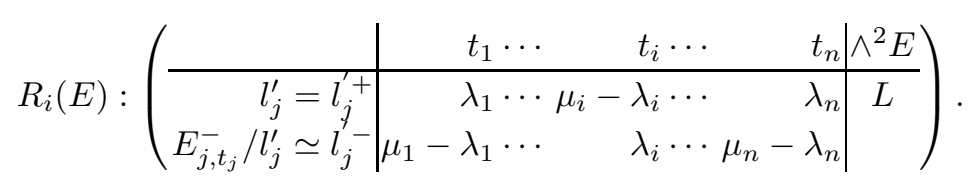

Now assume that $\operatorname{res}_{t_{i}}\left(\nabla_{L}\right) \in \mathbf{Z}$ for all $1 \leq i \leq n$.

Lemma 3.1. $\quad$ Assume that $\boldsymbol{\lambda}$ is not reducible (cf. Definition 2.3). Then $R_{i}$ induces an isomorphism

$$
R_{i}: M_{n}^{\alpha}(\mathbf{t}, \boldsymbol{\lambda}, L) \stackrel{\simeq}{\longrightarrow} M_{n}^{\alpha}\left(\mathbf{t}, \boldsymbol{\lambda}^{\prime}, L\right) .
$$

Proof. Since $\boldsymbol{\lambda}$ is not reducible, any $\left(E, \nabla_{E}, \varphi,\left\{l_{i}\right\}\right) \in M_{n}^{\boldsymbol{\alpha}}(\mathbf{t}, \boldsymbol{\lambda}, L)$ are irreducible (Lemma 2.1), so is $R_{i}(E)$. In particular $R_{i}(E)$ is $\boldsymbol{\alpha}$-stable. Therefore it induces a morphism of moduli spaces. Moreover it is obvious that $R_{i}^{2}=I d$, so it must be an isomorphism.

Later we will extend $R_{i}$ a birational map of the moduli spaces.

\section{§3.2. Birational transformations arising from elementary transformations}

Definition 3.1. Assume that $\boldsymbol{\alpha}$ is generic. An affine birational transformation of the family of moduli spaces $\pi_{n}: M_{n}^{\alpha}(L) \longrightarrow T_{n}^{\prime} \times \Lambda_{n}$ is a pair of maps $(\tilde{s}, s)$ consisting of a birational map $\tilde{s}: M_{n}^{\boldsymbol{\alpha}}(L) \cdots \longrightarrow M_{n}^{\boldsymbol{\alpha}}(L)$ and an affine transformation $s: \Lambda_{n} \longrightarrow \Lambda_{n}$ such that the following diagram commutes:

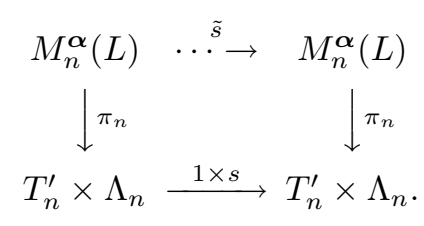

\subsubsection{The group $B L_{n}$}

Now we fix a determinant line bundle $\left(L, \nabla_{L}\right)=\left(\mathcal{O}_{\mathbf{P}^{1}}\left(-t_{n}\right), d\right)$ as in Remark 2.3 and consider the family of the moduli spaces $\pi_{n}: M_{n}^{\boldsymbol{\alpha}}\left(\mathcal{O}_{\mathbf{P}^{1}}\left(-t_{n}\right)\right) \longrightarrow$ $T_{n}^{\prime} \times \Lambda_{n}$. Let $\mathbf{e}_{i} \in \Lambda_{n}$ be the $i$-th standard base of $\Lambda_{n} \simeq \mathbf{C}^{n}$ and set $\boldsymbol{\lambda}=\left(\lambda_{1}, \ldots, \lambda_{n}\right) \in \Lambda_{n}$. We define a group $B L_{n}$ generated by the following affine automorphisms of $\Lambda_{n}$. 
(74)

$$
\left\{\begin{aligned}
t_{i}^{+}(\boldsymbol{\lambda}) & =\boldsymbol{\lambda}+\mathbf{e}_{i}=\left(\lambda_{1}, \ldots, \lambda_{i}+1, \ldots, \lambda_{n}\right) \\
t_{i, j}^{+}(\boldsymbol{\lambda}) & =\boldsymbol{\lambda}+\frac{1}{2}\left(\mathbf{e}_{i}+\mathbf{e}_{j}\right)=\left(\lambda_{1}, \ldots, \lambda_{i}+\frac{1}{2}, \ldots, \lambda_{j}+\frac{1}{2}, \ldots, \lambda_{n}\right) \\
t_{i, j}^{-}(\boldsymbol{\lambda}) & =\left(\lambda_{1}, \ldots,-\lambda_{i}+\frac{1}{2}, \ldots,-\lambda_{j}+\frac{1}{2}, \ldots, \lambda_{n}\right) \quad(1 \leq i<j \leq n-1) \\
t_{i, n}^{-}(\boldsymbol{\lambda}) & =\left(\lambda_{1}, \ldots,-\lambda_{i}+\frac{1}{2}, \ldots, \ldots, \ldots,-\lambda_{n}+\frac{3}{2}\right) \\
r_{i}(\boldsymbol{\lambda}) & =\left(\lambda_{1}, \ldots,-\lambda_{i}, \ldots, \lambda_{n}\right) \quad(1 \leq i \leq n-1) \\
r_{n}(\boldsymbol{\lambda}) & =\left(\lambda_{1}, \ldots, \lambda_{i}, \ldots, 1-\lambda_{n}\right) .
\end{aligned}\right.
$$

We can easily see the following relations.

$$
t_{i}^{+}=\left(t_{i, j}^{-} r_{i}\right)^{2}, \quad t_{i, j}^{+}=t_{i, j}^{-} r_{i} r_{j} .
$$

Therefore we can define the group $B L_{n}$ as

$$
B L_{n}=\left\langle t_{i, j}^{-},(1 \leq i<j \leq n), r_{k},(1 \leq k \leq n)\right\rangle .
$$

In [IIS2], we will show the following

Proposition 3.1. $\quad$ Every element s of the group $B L_{n}$ of affine transformations of $\Lambda_{n}$ can be lifted to a birational transformation

$$
\tilde{s}: M_{n}^{\boldsymbol{\alpha}}\left(\mathcal{O}_{\mathbf{P}^{1}}\left(-t_{n}\right)\right) \cdots \longrightarrow M_{n}^{\boldsymbol{\alpha}}\left(\mathcal{O}_{\mathbf{P}^{1}}\left(-t_{n}\right)\right)
$$

such that the pair $(\tilde{s}, s)$ becomes an affine birational transformation of the family of moduli spaces.

\section{$\S 4$. Moduli of Representations of Fundamental Groups}

\section{§4.1. The family of punctured projective lines and their}

\section{fundamental groups}

For $n \geq 3$, let us consider the space $T_{n}=\left\{\left(t_{1}, \ldots, t_{n}\right) \in\left(\mathbf{P}^{1}\right)^{n} \mid t_{i} \neq\right.$ $\left.t_{j},(i \neq j)\right\}$ and its open subset

$$
W_{n}=\left\{\left(t_{1}, \ldots, t_{n}\right) \in \mathbf{C}^{n} \quad \mid \quad t_{i} \neq t_{j},(i \neq j)\right\} .
$$

Setting $D(\mathbf{t})=t_{1}+\cdots+t_{n}$ for each $\mathbf{t}=\left(t_{1}, \ldots, t_{n}\right) \in T_{n}$, we denote by

$$
\Gamma_{n, \mathbf{t}}:=\pi_{1}\left(\mathbf{P}^{1} \backslash D(\mathbf{t}), *\right),
$$




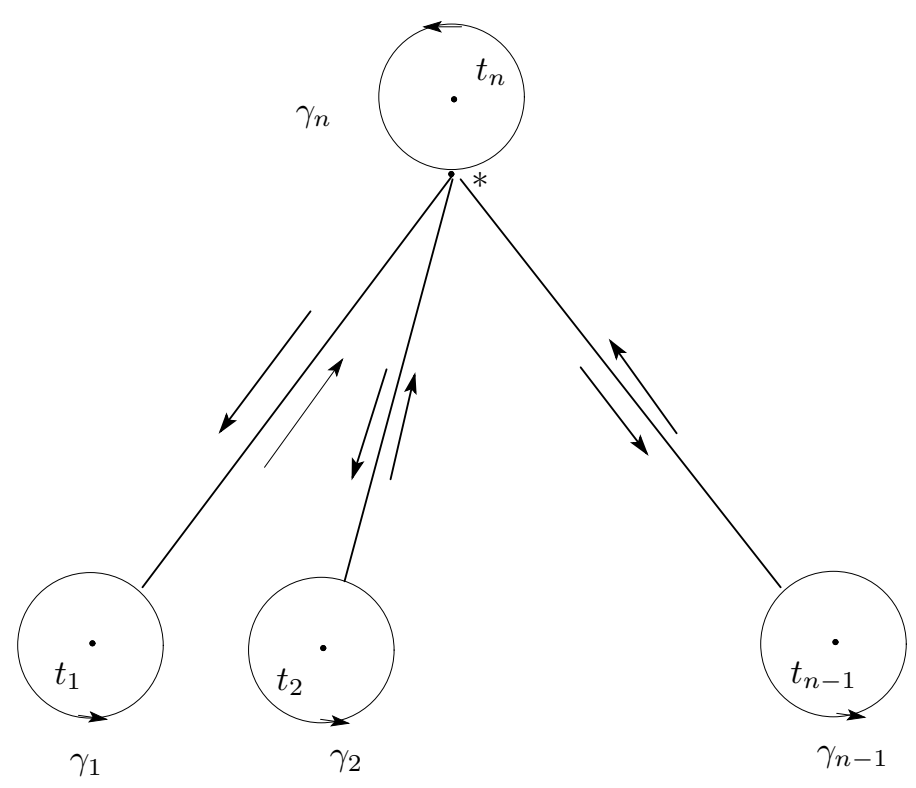

Figure 4. Canonical generators of $\pi_{1}\left(\mathbf{P}^{1} \backslash D(\mathbf{t}), *\right)$.

the fundamental group of $\mathbf{P}^{1} \backslash D(\mathbf{t})$ with the base point $*$ which we take very near to $t_{n}$. It is easy to see that $\Gamma_{n, \mathbf{t}}$ is generated by $\gamma_{1}, \ldots, \gamma_{n-1}, \gamma_{n}$ in Figure 4 with one relation $\gamma_{1} \gamma_{2} \cdots \gamma_{n}=1$. This set of generators $\gamma_{1}, \ldots, \gamma_{n}$ is called canonical generators of $\Gamma_{n, \mathbf{t}}$ with respect to the ordered $n$-points $\mathbf{t}$.

For each $i, 1 \leq i \leq n$, we define a divisor $\Sigma_{n, i}$ of $\mathbf{P}^{1} \times T_{n}$ as

$$
\Sigma_{n, i}=\left\{\left(z,\left(t_{1}, \ldots, t_{n}\right)\right) \in \mathbf{P}^{1} \times T_{n} \mid z=t_{i}\right\} .
$$

Setting $\mathcal{P}_{n}:=\left(\mathbf{P}^{1} \times T_{n}\right) \backslash\left(\cup_{i=1}^{n} \Sigma_{n, i}\right) \simeq T_{n+1}$, we obtain a natural projection map which induces a smooth morphism

$$
\tau_{n}: \mathcal{P}_{n} \longrightarrow T_{n}
$$

whose fiber $\mathcal{P}_{n, \mathbf{t}}$ over $\mathbf{t}=\left(t_{1}, \ldots, t_{n}\right)$ is $\mathbf{P}^{1} \backslash D(\mathbf{t})$. The family $\tau_{n}: \mathcal{P}_{n} \longrightarrow T_{n}$ in (81) is called the universal family of n-punctured lines.

By the universal covering map $\tilde{T}_{n} \longrightarrow T_{n}$, we can extend the family

$$
\begin{gathered}
\tilde{\mathcal{P}}_{n} \longrightarrow \mathcal{P}_{n} \\
\tilde{\tau}_{n} \downarrow \\
\tau_{n} \downarrow \\
\tilde{T}_{n} \longleftarrow T_{n},
\end{gathered}
$$


where we set $\tilde{\mathcal{P}}_{n}=\mathcal{P}_{n} \times_{T_{n}} \tilde{T}_{n}$.

Fix a base point $\mathbf{t}_{0} \in T_{n}$ and consider the fundamental group $\pi_{1}\left(T_{n}, \mathbf{t}_{0}\right)$. The natural $n$-th projection $h_{n}: T_{n} \longrightarrow \mathbf{P}^{1}\left(\left(t_{1}, \ldots, t_{n}\right) \mapsto t_{n}\right)$ gives a structure of fiber bundle over $\mathbf{P}^{1}$ whose fiber at $t_{n}=\infty$ is isomorphic to $W_{n-1}$. By using the exact sequence of fundamental groups for fiber bundles, one can see that there exists an isomorphism

$$
\pi_{1}\left(T_{n}, \mathbf{t}_{0}\right) \simeq \pi_{1}\left(W_{n-1}, \mathbf{t}_{0}\right) .
$$

On the other hand, it is well known that the fundamental group $\pi_{1}\left(W_{n-1}, \mathbf{t}_{0}\right)$ is isomorphic to the pure braid group $P B_{n-1}$ of $n-1$ strings. Therefore the pure braid group $P B_{n-1}$ acts on the universal covering $\tilde{T}_{n}$ and also the typical fiber $\mathcal{P}_{n, \mathbf{t}_{0}}$ of $\tilde{\tau}_{n}$ in (82).

Moreover the fiber bundle $\hat{\pi}_{n}: \tilde{\mathcal{P}}_{n} \longrightarrow \tilde{T}_{n}$ becomes trivial, that is, there exists a diffeomorphism $\tilde{\mathcal{P}}_{n} \stackrel{\simeq}{\longrightarrow} \mathcal{P}_{n, \mathbf{t}^{0}} \times \tilde{T}_{n}$ such that the following diagram commutes:

$$
\tilde{\mathcal{P}}_{n} \underset{\tilde{\tau}_{n} \searrow}{\stackrel{\simeq}{\longrightarrow}} \mathcal{P}_{n, \mathbf{t}_{0}} \times \tilde{T}_{n}
$$

By using the isomorphism, for every $\tilde{\mathbf{t}} \in \tilde{T}_{n}$, we can obtain the isomorphism of fundamental groups

$$
\pi_{1}\left(\tilde{\mathcal{P}}_{n, \tilde{\mathbf{t}}^{*}}\right) \simeq \pi_{1}\left(\mathcal{P}_{n, \mathbf{t}_{0}}, *\right)=\Gamma_{n, \mathbf{t}_{0}}
$$

as well as the identification of canonical generators $\gamma_{1}, \ldots, \gamma_{n}$ in Figure 4 . The action of the pure braid group $P B_{n-1}$ on the fiber bundle $\hat{\pi}_{n}: \tilde{\mathcal{P}}_{n} \longrightarrow \tilde{T}_{n}$ induces an action on canonical generators of $\Gamma_{n, \mathbf{t}_{0}}$, which can be written in a very explicit way. (For example for the case of $n=4$, see [Iw3], [Iw4]).

\section{§4.2. The moduli space of $S L_{2}(\mathbf{C})$-representations}

Definition 4.1. An $S L_{2}(\mathbf{C})$-representation of the fundamental group $\Gamma_{n, \mathbf{t}}=\pi_{1}\left(\mathcal{P}_{n, \mathbf{t}}, *\right)$ of $\mathcal{P}_{n, \mathbf{t}}=\mathbf{P}^{1} \backslash D(\mathbf{t})$ is a group homomorphism

$$
\rho: \Gamma_{n, \mathbf{t}}=\pi_{1}\left(\mathcal{P}_{n, \mathbf{t}}, *\right) \longrightarrow S L_{2}(\mathbf{C}) .
$$

We denote by $\operatorname{Hom}\left(\Gamma_{n, \mathbf{t}}, S L_{2}(\mathbf{C})\right)$ the set of all $S L_{2}(\mathbf{C})$-representations of $\Gamma_{n, \mathbf{t}}$. If we fix a set of canonical generators $\gamma_{1}, \ldots, \gamma_{n}$ of $\Gamma_{n, \mathbf{t}}$ as in Figure 4, we have the identification

$$
\operatorname{Hom}\left(\Gamma_{n, \mathbf{t}}, S L_{2}(\mathbf{C})\right)=S L_{2}(\mathbf{C})^{n-1}
$$

given by $\rho \mapsto\left(\rho\left(\gamma_{i}\right)\right)$ for $i=1, \ldots, n-1$. 


\section{Definition 4.2 .}

(1) Two $S L_{2}(\mathbf{C})$-representations $\rho_{1}, \rho_{2}$ are isomorphic to each other, if and only if there exists a matrix $P \in S L_{2}(\mathbf{C})$ such that

$$
\rho_{2}(\gamma)=P^{-1} \cdot \rho_{2}(\gamma) \cdot P \quad \text { for all } \gamma \in \pi_{1}\left(\tilde{\mathcal{P}}_{n, \mathbf{t}}, *\right) .
$$

(2) A semisimplification of a representation $\rho$ is an associated graded of the composition series of $\rho$.

(3) Two $S L_{2}(\mathbf{C})$-representation is said to be Jordan equivalent if their semisimplifications are isomorphic.

Fixing $\mathbf{t}_{0} \in T_{n}$ and canonical generators $\gamma_{1}, \ldots, \gamma_{n}$ of $\Gamma_{n, \mathbf{t}_{0}}$ and using the isomorphism in (85), for any $\mathbf{t} \in \tilde{T}_{n}$, we fix an identification

$$
\operatorname{Hom}\left(\Gamma_{n, \mathbf{t}}, S L_{2}(\mathbf{C})\right) \stackrel{\simeq}{\longrightarrow} S L_{2}(\mathbf{C})^{n-1}
$$

by $\rho \mapsto\left(\rho\left(\gamma_{1}\right), \ldots, \rho\left(\gamma_{n-1}\right)\right)$.

Let $R_{n-1}$ denote the affine coordinate ring of $S L_{2}(\mathbf{C})^{n-1}$ and consider the simultaneous action of $S L_{2}(\mathbf{C})$ on $S L_{2}(\mathbf{C})^{n-1}$ as

$$
\left(M_{1}, \cdots, M_{n-1}\right) \mapsto\left(P^{-1} M_{1} P, \cdots, P^{-1} M_{n-1} P\right) .
$$

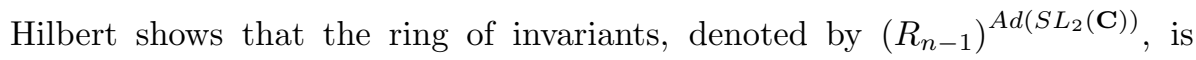
finitely generated. The following lemma is due to Simpson [Sim2],

Lemma 4.1 ([Mum], [Proposition 6.1, [Sim2]]). For any $\mathbf{t} \in \tilde{T}_{n}$, under the identification (87), there exists the universal categorical quotient map

$\Phi_{n}: \operatorname{Hom}\left(\Gamma_{n, \mathbf{t}}, S L_{2}(\mathbf{C})\right) \simeq S L_{2}(\mathbf{C})^{n-1} \longrightarrow \mathcal{R}\left(\mathcal{P}_{n, \mathbf{t}}\right)=S L_{2}(\mathbf{C})^{n-1} / A d\left(S L_{2}(\mathbf{C})\right)$ where

$$
\mathcal{R}\left(\mathcal{P}_{n, \mathbf{t}}\right)=\operatorname{Spec}\left[\left(R_{n-1}\right)^{\operatorname{Ad}\left(S L_{2}(\mathbf{C})\right)}\right] .
$$

The closed points of $\mathcal{R}\left(\mathcal{P}_{n, \mathbf{t}}\right)$ represent the Jordan equivalence classes of $S L_{2}(\mathbf{C})$-representations of $\Gamma_{n, \mathbf{t}}$. We say that $\mathcal{R} \mathcal{P}_{n}=\mathcal{R}\left(\mathcal{P}_{n, \mathbf{t}}\right)$ is the moduli space of $S L_{2}(\mathbf{C})$-representation of $\pi_{1}\left(\mathbf{P}^{1} \backslash \Sigma(\mathbf{t})\right)$.

Remark 4.1. Lemma 4.1 says that the set $\mathcal{R}\left(\mathcal{P}_{n, \mathbf{t}}\right)$ of Jordan equivalence classes of $S L_{2}(\mathbf{C})$-representations admits a natural structure of an affine scheme. Moreover, it is easy to see that the moduli stack of isomorphism classes of $S L_{2}(\mathbf{C})$-representations has no natural scheme structure. 
Remark 4.2. It is obvious that the algebraic structure or complex structure of the moduli space $\mathcal{R}\left(\mathcal{P}_{n, \mathbf{t}}\right)$ does not depend on $\mathbf{t} \in \tilde{T}_{n}$. However in order to define the isomorphism $\operatorname{Hom}\left(\Gamma_{n, \mathbf{t}}, S L_{2}(\mathbf{C})\right) \simeq S L_{2}(\mathbf{C})^{n-1}$ we have to fix canonical generators of $\Gamma_{n, \mathbf{t}}=\pi_{1}\left(\mathbf{P}^{1} \backslash D(\mathbf{t})\right)$. Since the pure braid group $P B_{n-1}:=\pi_{1}\left(T_{n}, *\right)$ acts on the sets of generators of $\Gamma_{n, \mathbf{t}}$ and hence acts on $\mathcal{R}\left(\mathcal{P}_{n, \mathbf{t}}\right)$. This action is called the topological nonlinear monodromy action of the pure braid group $P B_{n-1}:=\pi_{1}\left(T_{n}, *\right)$. (Cf. [DM], [Iw3], [Iw4]).

In our case, we can describe the categorical quotient $\operatorname{Spec}\left[\left(R_{n-1}\right)^{\operatorname{Ad}\left(S L_{2}(\mathbf{C})\right)}\right]$ more explicitly. Denote the coordinate ring $R_{n-1}$ of $S L_{2}(\mathbf{C})^{n-1}$ by

$$
R_{n-1}=\mathbf{C}\left[a_{i}, b_{i}, c_{i}, d_{i}\right] /\left(a_{i} d_{i}-b_{i} c_{i}-1\right) \quad i=1, \ldots, n-1
$$

where $M_{i}=\left(\begin{array}{ll}a_{i} & b_{i} \\ c_{i} & d_{i}\end{array}\right)$.

The following Proposition follows from the fundamental theorem for matrix invariants. (See [Theorem 2, Theorem 7, [For]] or [Theorem 1.3, [P]]).

\section{Proposition 4.1.}

$$
\left(R_{n-1}\right)^{\operatorname{Ad}\left(S L_{2}(\mathbf{C})\right)}=\mathbf{C}\left[\operatorname{Tr}\left(M_{i_{1}} M_{i_{2}} \cdots M_{i_{k}}\right), 1 \leq i_{1}, \ldots, i_{k} \leq n-1\right] .
$$

Moreover, the elements $\operatorname{Tr}\left(M_{i_{1}} M_{i_{2}} \cdots M_{i_{k}}\right)$ of degree $k \leq 3$ generate the invariant ring, that is,

$$
\left(R_{n-1}\right)^{\operatorname{Ad}\left(S L_{2}(\mathbf{C})\right)}=\mathbf{C}\left[\operatorname{Tr}\left(M_{i}\right), \operatorname{Tr}\left(M_{i} M_{j}\right), \operatorname{Tr}\left(M_{i} M_{j} M_{k}\right) \mid 1 \leq i, j, k \leq n-1\right] .
$$

Let us set

$$
a_{i}=\operatorname{Tr}\left(M_{i}\right) \quad \text { for } \quad 1 \leq i \leq n,
$$

which are elements of $\left(R_{n-1}\right)^{A d\left(S L_{2}(\mathbf{C})\right)}$ and consider the subring $A_{n}=$ $\mathbf{C}\left[a_{1}, \ldots, a_{n}\right]$ of $\left(R_{n-1}\right)^{A d\left(S L_{2}(\mathbf{C})\right.}$. We have a natural morphism

$$
p_{n}: \mathcal{R}\left(\mathcal{P}_{n, \mathbf{t}}\right)=\operatorname{Spec}\left[\left(R_{n-1}\right)^{\operatorname{Ad}\left(S L_{2}(\mathbf{C})\right)}\right] \longrightarrow \mathcal{A}_{n}=\operatorname{Spec}\left[A_{n}\right] .
$$

\section{$\S 4.3$. Construction of the family of moduli spaces}

$$
\phi_{n}: \mathcal{R}_{n} \longrightarrow T_{n}^{\prime} \times \mathcal{A}_{n}
$$

Fix $\mathbf{t}_{0} \in T_{n}$ as the base point of fundamental group $\pi_{1}\left(T_{n}, \mathbf{t}_{0}\right)$ and fix canonical generators $\gamma_{1}, \ldots, \gamma_{n}$ of $\Gamma_{n, \mathbf{t}_{0}}$. Again taking the universal covering 
map $\tilde{T}_{n} \longrightarrow T_{n}$, we can obtain a trivialization (84) and isomorphisms of the fundamental groups (85). By using the isomorphisms, for each $\mathbf{t} \in \tilde{T}_{n}$, we obtain a canonical isomorphism

$$
\mathcal{R}\left(\mathcal{P}_{n, \mathbf{t}}\right) \simeq \mathcal{R}\left(\mathcal{P}_{n, \mathbf{t}_{0}}\right)
$$

Moreover the group $\pi_{1}\left(T_{n}, \mathbf{t}_{0}\right) \simeq P B_{n-1}$ acts on the variety $\mathcal{R}\left(\mathcal{P}_{n, \mathbf{t}_{0}}\right)$ as the group of nonlinear monodromies and hence defines the action on the product $\mathcal{R}\left(\mathcal{P}_{n, \mathbf{t}_{0}}\right) \times \tilde{T}_{n}$. Define the subgroup $\Gamma_{n-1}$ of $\pi_{1}\left(T_{n}, \mathbf{t}_{0}\right)$ as a kernel of the natural homomorphism $\pi_{1}\left(T_{n}, \mathbf{t}_{0}\right) \longrightarrow \operatorname{Aut}\left(\mathbf{C}\left[a_{1}, \ldots, a_{n}\right]\right)$. It is easy to see that $\Gamma_{n-1}$ is a subgroup of $\pi_{1}\left(T_{n}, \mathbf{t}_{0}\right)$ of finite index, so defining as $T_{n}^{\prime}=\tilde{T}_{n} / \Gamma_{n-1}$ we obtain the finite étale covering

$$
T_{n}^{\prime}:=\tilde{T}_{n} / \Gamma_{n-1} \longrightarrow T_{n} .
$$

Consider the natural action of $\Gamma_{n-1}$ on the product $\tilde{T}_{n} \times \mathcal{R}\left(\mathcal{P}_{n, \mathbf{t}_{0}}\right)$. The natural map $1 \times p_{n}: \tilde{T}_{n} \times \mathcal{R}\left(\mathcal{P}_{n, \mathbf{t}_{0}}\right) \longrightarrow \tilde{T}_{n} \times \mathcal{A}_{n}$ is clearly equivariant with respect to the action of $\Gamma_{n-1}$, where $\Gamma_{n-1}$ acts on $\mathcal{A}_{n}$ as the identity map. Setting

$$
\mathcal{R}_{n}=\tilde{T}_{n} \times \mathcal{R}\left(\mathcal{P}_{n, \mathbf{t}_{0}}\right) / \Gamma_{n-1}
$$

we obtain a morphism

$$
\phi_{n}: \mathcal{R}_{n} \longrightarrow T_{n}^{\prime} \times \mathcal{A}_{n}
$$

which is said to be the family of the moduli spaces of $S L_{2}$-representations of the fundamental group. The fiber of $\phi_{n}$ at $(\mathbf{t}, \mathbf{a})$ is given by the affine subscheme of $\mathcal{R}_{n}$

$$
\phi_{n}^{-1}(\mathbf{t}, \mathbf{a})=\mathcal{R}\left(\mathcal{P}_{n, \mathbf{t}}\right)_{\mathbf{a}}:=\left\{[\rho] \in \mathcal{R}\left(\mathcal{P}_{n, \mathbf{t}}\right) \mid \operatorname{Tr}\left[\rho\left(\gamma_{i}\right)\right]=a_{i}, 1 \leq i \leq n\right\} .
$$

Since $a_{i}$ determines the eigenvalues of monodromy matrix $\rho\left(\gamma_{i}\right)$, a may be considered as the set of spectral of local monodromies. Hence the space $\mathcal{R}\left(\mathcal{P}_{n, \mathbf{t}}\right)_{\mathbf{a}}$ is said to be the moduli space of isospectral $S L_{2}$-representations. Note that though the moduli space $M_{n}^{\boldsymbol{\alpha}}(\mathbf{t}, \boldsymbol{\lambda}, L)$ is smooth for all $(\mathbf{t}, \boldsymbol{\lambda})$ if $\mathbf{a}$ is special in the sense of Definition 1.1 the affine scheme $\mathcal{R}\left(\mathcal{P}_{n, \mathbf{t}}\right)_{\mathbf{a}}$ has singularities.

In $\S 8$, we will prove the following

Proposition 4.2. For any $\mathbf{a} \in \mathcal{A}_{n}$, the scheme $\mathcal{R}\left(\mathcal{P}_{n, \mathbf{t}}\right)_{\mathbf{a}}$ in $(97)$ is irreducible. 


\section{§4.4. The case of $n=4$}

Now we recall the explicit description of the invariant ring for $n=4$ due to Iwasaki ([Iw3], [Iw4]). We denote by $(i, j, k)$ a cyclic permutation of $(1,2,3)$. Then the invariant ring $\left(R_{3}\right)^{A d\left(S L_{2}(\mathbf{C})\right)}$ is generated by

$$
\begin{array}{ll}
x_{i}=\operatorname{Tr}\left[M_{j} M_{k}\right] & \text { for } i=1,2,3 \\
a_{i}=\operatorname{Tr}\left[M_{i}\right] & \text { for } i=1,2,3 \\
a_{4}=\operatorname{Tr}\left[M_{1} M_{2} M_{3}\right] &
\end{array}
$$

The following proposition is proved in [Iw4].

Proposition 4.3. The invariant ring $\left(R_{3}\right)^{A d\left(S L_{2}(\mathbf{C})\right)}$ is generated by seven elements $x_{1}, x_{2}, x_{3}, a_{1}, a_{2}, a_{3}, a_{4}$ and there exists a relation

$$
f(\mathbf{x}, \mathbf{a})=x_{1} x_{2} x_{3}+x_{1}^{2}+x_{2}^{2}+x_{3}^{2}-\theta_{1}(\mathbf{a}) x_{1}-\theta_{2}(\mathbf{a}) x_{2}-\theta_{3}(\mathbf{a}) x_{3}+\theta_{4}(\mathbf{a})
$$

where we set

(100) $\theta_{i}(\mathbf{a})=a_{i} a_{4}+a_{j} a_{k}, \quad(i, j, k)=$ a cyclic permutation of $(1,2,3)$,

(101) $\theta_{4}(\mathbf{a})=a_{1} a_{2} a_{3} a_{4}+a_{1}^{2}+a_{2}^{2}+a_{3}^{2}+a_{4}^{2}-4$.

Therefore we have an isomorphism

$$
\left(R_{3}\right)^{\operatorname{Ad}\left(S L_{2}(\mathbf{C})\right)} \simeq \mathbf{C}\left[x_{1}, x_{2}, x_{3}, a_{1}, a_{2}, a_{3}, a_{4}\right] /(f(\mathbf{z}, \mathbf{a})) .
$$

Recall that fixing canonical generators of the fundamental group, for any $\mathbf{t} \in \tilde{T}_{4}$, the categorical quotient $\mathcal{R}_{4, \mathbf{t}}$ is given by $\mathcal{R}\left(\mathcal{P}_{4, \mathbf{t}}\right):=$ $\operatorname{Spec}\left[\left(R_{3}\right)^{\operatorname{Ad}\left(S L_{2}(\mathbf{C})\right)}\right] \simeq \operatorname{Spec}[\mathbf{C}[\mathbf{x}, \mathbf{a}] /(f(\mathbf{x}, \mathbf{a}))]$. Setting $\mathcal{A}_{4}=\mathbf{C}^{4}=$ $\operatorname{Spec}\left[\mathbf{C}\left[a_{1}, \ldots, a_{4}\right]\right]$, as in (93) we have a surjective morphism

$$
p_{4}: \mathcal{R}\left(\mathcal{P}_{4, \mathbf{t}}\right)=\operatorname{Spec}[\mathbf{C}[\mathbf{x}, \mathbf{a}] /(f(\mathbf{x}, \mathbf{a}))] \longrightarrow \mathcal{A}
$$

whose fiber at $\mathbf{a} \in \mathcal{A}$ is an affine cubic hypersurface in $\mathbf{C}^{3}$

$$
\mathcal{R}\left(\mathcal{P}_{4, \mathbf{t}}\right)_{\mathbf{a}} \simeq\left\{\left(x_{1}, x_{2}, x_{3}\right) \in \mathbf{C}^{3} \mid f(\mathbf{x}, \mathbf{a})=0\right\} \subset \mathbf{C}^{3} .
$$

Therefore, the family in (96) $\phi_{4}: \mathcal{R}_{4} \longrightarrow T_{4}^{\prime} \times \mathcal{A}_{4}$ is a family of affine cubic hypersurfaces in $\mathbf{C}^{3}$.

The subgroup $\Gamma_{3}$ of $\pi_{1}\left(T_{4}, \mathbf{t}_{0}\right)$ acts both on the space $\mathcal{R}\left(\mathcal{P}_{4, \mathbf{t}}\right)$ and the space $\mathcal{R}\left(\mathcal{P}_{4, \mathbf{t}}\right)_{\mathbf{a}}$ as nonlinear monodromies. Iwasaki [Iw3] showed the following 
Proposition 4.4. There exists a one-to-one correspondence between the set of fixed points of the action of $\Gamma_{3}$ on $\mathcal{R}\left(\mathcal{P}_{4, \mathbf{t}}\right)_{\mathbf{a}}$ and the set of singular points on the affine cubic hypersurface $\mathcal{R}\left(\mathcal{P}_{4, \mathbf{t}}\right)_{\mathbf{a}}$.

\section{$\S 5$. Construction of the Moduli Space $\overline{M_{n}^{\alpha^{\prime} \boldsymbol{\beta}}}(\mathbf{t}, \boldsymbol{\lambda}, L)$ and Proof of Theorem 2.1, (1)}

\section{$\S 5.1$. Translation of the definition of parabolic $\phi$-connection}

In this section, we will translate the definition of parabolic $\phi$-connection, since it is rather convenient to generalize the definition for the construction of the moduli space.

Let $X$ be a smooth projective curve over $\mathbf{C}$ and $D$ be an effective divisor on $X$.

We define an $\mathcal{O}_{X}$-bimodule structure on $\Lambda_{D}^{1}=\mathcal{O}_{X} \oplus\left(\Omega_{X}^{1}(D)\right)^{\vee}$ by

$$
\begin{aligned}
(a, v) f & :=(f a+\langle v, d f\rangle, f v) \\
f(a, v) & :=(f a, f v)
\end{aligned}
$$

for $a, f \in \mathcal{O}_{X}$ and $v \in\left(\Omega_{X}^{1}(D)\right)^{\vee}$, where $\langle\rangle:,\left(\Omega_{X}^{1}(D)\right)^{\vee} \times \Omega_{X}^{1}(D) \rightarrow \mathcal{O}_{X}$ is the canonical pairing.

Definition 5.1. A parabolic $\Lambda_{D}^{1}$-triple $\left(E_{1}, E_{2}, \Phi, F_{*}\left(E_{1}\right)\right)$ on $X$ consists of two vector bundles $E_{1}, E_{2}$ on $X$, a left $\mathcal{O}_{X}$-homomorphism $\Phi: \Lambda_{D}^{1} \otimes_{\mathcal{O}_{X}}$ $E_{1} \rightarrow E_{2}$ and a filtration of coherent subsheaves: $E_{1}=F_{1}\left(E_{1}\right) \supset F_{2}\left(E_{1}\right) \supset$ $\cdots \supset F_{l}\left(E_{1}\right) \supset F_{l+1}\left(E_{1}\right)=E_{1}(-D)$.

Remark 5.1. Assume that two vector bundles $E_{1}, E_{2}$ on $X$ are given. Then giving morphisms $\phi: E_{1} \rightarrow E_{2}, \nabla: E_{1} \rightarrow E_{2} \otimes \Omega_{X}^{1}(D)$ satisfying $\phi(f a)=f \phi(a), \nabla(f a)=\phi(a) \otimes d f+f \nabla(a)$ for $f \in \mathcal{O}_{X}, a \in E_{1}$ is equivalent to giving a left $\mathcal{O}_{X}$-homomorphism $\Phi: \Lambda_{D}^{1} \otimes_{\mathcal{O}_{X}} E_{1} \rightarrow E_{2}$.

Definition 5.2. A parabolic $\Lambda_{D}^{1}$-triple $\left(E_{1}^{\prime}, E_{2}^{\prime}, \Phi^{\prime}, F_{*}\left(E_{1}^{\prime}\right)\right)$ is said to be a parabolic $\Lambda_{D}^{1}$-subtriple of $\left(E_{1}, E_{2}, \Phi, F_{*}\left(E_{1}\right)\right)$ if $E_{1}^{\prime} \subset E_{1}, E_{2}^{\prime} \subset E_{2}$, $\left.\Phi\right|_{\Lambda_{D}^{1} \otimes E_{1}^{\prime}}=\Phi^{\prime}$ and $F_{i}\left(E_{1}^{\prime}\right) \subset F_{i}\left(E_{1}\right)$ for any $i$.

Fix rational numbers $0 \leq \alpha_{1}^{\prime}<\alpha_{2}^{\prime}<\cdots<\alpha_{l}^{\prime}<\alpha_{l+1}^{\prime}=1$ and positive integers $\beta_{1}, \beta_{2}$. We write $\boldsymbol{\alpha}^{\prime}=\left(\alpha_{1}^{\prime}, \ldots, \alpha_{l}^{\prime}\right)$ and $\boldsymbol{\beta}=\left(\beta_{1}, \beta_{2}\right)$. We also fix an ample line bundle $\mathcal{O}_{X}(1)$ and a rational number $\gamma$ with $\gamma \gg 0$. 
Definition 5.3. For a parabolic $\Lambda_{D}^{1}$-triple $\left(E_{1}, E_{2}, \Phi, F_{*}\left(E_{1}\right)\right)$, we put $\mu\left(E_{1}, E_{2}, \Phi, F_{*}\left(E_{1}\right)\right)$
$:=\frac{\beta_{1} \operatorname{deg} E_{1}(-D)+\beta_{2} \operatorname{deg} E_{2}-\beta_{2} \gamma \operatorname{deg} \mathcal{O}_{X}(1) \operatorname{rank} E_{2}+\sum_{i=1}^{l} \beta_{1} \alpha_{i}^{\prime} \operatorname{length}\left(F_{i}\left(E_{1}\right) / F_{i+1}\left(E_{1}\right)\right)}{\beta_{1} \operatorname{rank} E_{1}+\beta_{2} \operatorname{rank} E_{2}}$.

Definition 5.4. Assume that $\gamma$ is sufficiently large. A parabolic $\Lambda_{D^{-}}^{1}$ triple $\left(E_{1}, E_{2}, \Phi, F_{*}\left(E_{1}\right)\right)$ is $\left(\boldsymbol{\alpha}^{\prime}, \boldsymbol{\beta}\right)$-stable (resp. $\left(\boldsymbol{\alpha}^{\prime}, \boldsymbol{\beta}\right)$-semistable) if for any non-zero proper parabolic $\Lambda_{D}^{1}$-subtriple $\left(E_{1}^{\prime}, E_{2}^{\prime}, \Phi^{\prime}, F_{*}\left(E_{1}^{\prime}\right)\right)$ of $\left(E_{1}, E_{2}, \Phi\right.$, $\left.F_{*}\left(E_{1}\right)\right)$, the inequality

$$
\mu\left(E_{1}^{\prime}, E_{2}^{\prime}, \Phi^{\prime}, F_{*}\left(E_{1}^{\prime}\right)\right) \underset{(\text { resp. } \leq)}{<} \mu\left(E_{1}, E_{2}, \Phi, F_{*}\left(E_{1}\right)\right)
$$

holds. (If we fix a weight $\left(\boldsymbol{\alpha}^{\prime}, \boldsymbol{\beta}\right), "\left(\boldsymbol{\alpha}^{\prime}, \boldsymbol{\beta}\right)$-stable (resp. $\left(\boldsymbol{\alpha}^{\prime}, \boldsymbol{\beta}\right)$-semistable)" may be abbreviated to "stable (resp. semistable)" for simplicity.)

Let $S$ be a connected noetherian scheme and $\pi_{S}: \mathcal{X} \rightarrow S$ be a smooth projective morphism whose geometric fibers are curves of genus $g$. Let $\mathcal{D} \subset$ $\mathcal{X}$ be an effective Cartier divisor which is flat over $S$. A similar formula to

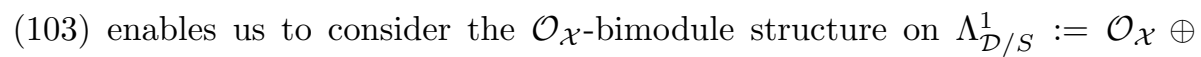
$\left(\Omega_{\mathcal{X} / S}^{1}(\mathcal{D})\right)^{\vee}$.

Fix rational numbers $0 \leq \alpha_{1}^{\prime}<\alpha_{2}^{\prime}<\cdots<\alpha_{l}^{\prime}<\alpha_{l+1}^{\prime}=1$, positive integers $r, d,\left\{d_{i}\right\}_{1 \leq i \leq l}, \beta_{1}, \beta_{2}, \gamma$ with $\gamma \gg 0$.

Definition 5.5. We define the moduli functor $\overline{\mathcal{M}_{\mathcal{X} / S}^{\mathcal{D}, \boldsymbol{\alpha}^{\prime}, \boldsymbol{\beta}, \gamma}}\left(r, d,\left\{d_{i}\right\}\right)$ of the category of locally noetherian schemes over $S$ to the category of sets by

$$
\overline{\mathcal{M}_{\mathcal{X} / S}^{\mathcal{D}, \boldsymbol{\alpha}^{\prime}, \boldsymbol{\beta}, \gamma}}\left(r, d,\left\{d_{i}\right\}\right)(T):=\left\{\left(E_{1}, E_{2}, \Phi, F_{*}\left(E_{1}\right)\right)\right\} / \sim,
$$

where $T$ is a locally noetherian scheme over $S$ and

(1) $E_{1}, E_{2}$ are vector bundles on $\mathcal{X} \times_{S} T$ such that for any geometric point $s$ of $T, \operatorname{rank}\left(E_{1}\right)_{s}=\operatorname{rank}\left(E_{2}\right)_{s}=r, \operatorname{deg}\left(E_{1}\right)_{s}=\operatorname{deg}\left(E_{2}\right)_{s}=d$,

(2) $\Phi: \Lambda_{\mathcal{D} / S}^{1} \otimes_{\mathcal{O}_{\mathcal{X}}} E_{1} \rightarrow E_{2}$ is a homomorphism of left $\mathcal{O}_{\mathcal{X} \times{ }_{S} T}$-modules,

(3) $E_{1}=F_{1}\left(E_{1}\right) \supset F_{2}\left(E_{1}\right) \supset \cdots \supset F_{l}\left(E_{1}\right) \supset F_{l+1}\left(E_{1}\right)=E_{1}\left(-\mathcal{D}_{T}\right)$ is a filtration of $E_{1}$ by coherent subsheaves such that each $E_{1} / F_{i+1}\left(E_{1}\right)$ is flat over $T$ and for any geometric point $s$ of $T$, length $\left(\left(E_{1} / F_{i+1}\left(E_{1}\right)\right)_{s}\right)=d_{i}$,

(4) for any geometric point $s$ of $S$, the parabolic $\Lambda_{\mathcal{D}_{s}}^{1}$-triple $\left(\left(E_{1}\right)_{s},\left(E_{2}\right)_{s}, \Phi_{s}\right.$, $\left.F_{*}\left(E_{1}\right)_{s}\right)$ is stable (that is, $\left(\boldsymbol{\alpha}^{\prime}, \boldsymbol{\beta}\right)$-stable). 
$\left(E_{1}, E_{2}, \Phi, F_{*}\left(E_{1}\right)\right) \sim\left(E_{1}^{\prime}, E_{2}^{\prime}, \Phi^{\prime}, F_{*}\left(E_{1}^{\prime}\right)\right)$ if there exist a line bundle $\mathcal{L}$ on $T$ and isomorphisms $\sigma_{j}: E_{j} \stackrel{\sim}{\rightarrow} E_{j}^{\prime} \otimes \mathcal{L}$ for $j=1,2$ such that $\sigma_{1}\left(F_{i+1}\left(E_{1}\right)\right)=$ $F_{i+1}\left(E_{1}^{\prime}\right) \otimes \mathcal{L}$ for any $i$ and the diagram

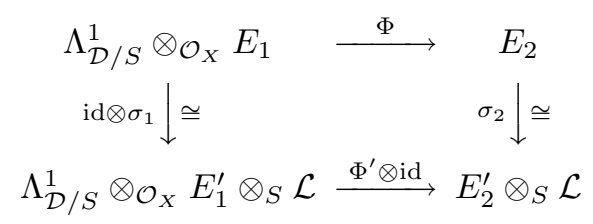

commutes.

We call $\left(E_{1}, E_{2}, \Phi, F_{*}\left(E_{1}\right)\right)$ a flat family of parabolic $\Lambda_{\mathcal{D}_{T} / T^{-t r i p l e s ~ o n ~}}^{1}$ $\mathcal{X}_{T} \times T$ over $T$ if it satisfies the above conditions (1), (2) and (3).

\section{$\S 5.2$. Boundedness and Openness of stability}

Proposition 5.1. The family of geometric points of $\overline{\mathcal{M}_{\mathcal{X} / S}^{\mathcal{D}, \boldsymbol{\alpha}^{\prime}, \boldsymbol{\beta}, \gamma}}\left(r, d,\left\{d_{i}\right\}\right)$ is bounded.

Proof. Take any geometric point $\left(E_{1}, E_{2}, \Phi, F_{*}\left(E_{1}\right)\right) \quad \in$
$\mathcal{M}_{\mathcal{X} / S}^{\mathcal{D}, \boldsymbol{\alpha}^{\prime}, \boldsymbol{\beta}, \gamma}\left(r, d,\left\{d_{i}\right\}\right)(K)$. By Serre duality, we have

$$
H^{1}\left(\mathcal{X}_{K}, E_{1}(m-1)\right)=\operatorname{Hom}\left(E_{1}, \omega_{\mathcal{X}_{K}}(1-m)\right)^{\vee} .
$$

Take any nonzero homomorphism $f: E_{1} \rightarrow \omega_{\mathcal{X}_{K}}(1-m)$. Then $\left(\operatorname{ker} f, E_{2},\left.\Phi\right|_{\operatorname{ker} f}\right.$, $F_{*}\left(E_{1}\right) \cap$ ker $\left.f\right)$ becomes a parabolic $\Lambda_{\mathcal{D}_{K}}^{1}$-subtriple of $\left(E_{1}, E_{2}, \Phi, F_{*}\left(E_{1}\right)\right)$. Thus we must have the inequality

$$
\mu\left(\operatorname{ker} f, E_{2},\left.\Phi\right|_{\operatorname{ker} f}, F_{*}\left(E_{1}\right) \cap \operatorname{ker} f\right)<\mu\left(E_{1}, E_{2}, \Phi, F_{*}\left(E_{1}\right)\right) .
$$

Since $\operatorname{deg}(\operatorname{ker} f) \geq \operatorname{deg} E_{1}+m-2 g+1$, we can find an integer $m$ which depends only on $r, d, d_{i}, \boldsymbol{\beta}, \boldsymbol{\alpha}^{\prime}, \gamma, \mathcal{X}$ and $\mathcal{D}$ such that $\operatorname{Hom}\left(E_{1}, \omega_{\mathcal{X}_{K}}(1-m)\right)=0$. Then all $E_{1}$ become $m$-regular.

Similarly we can find an integer $m^{\prime}$ such that $E_{2}$ are all $m^{\prime}$-regular. Then the family of $\left(E_{1}, E_{2}\right)$ is bounded and the boundedness of the family of $\left(E_{1}, E_{2}, \Phi, F_{*}\left(E_{1}\right)\right)$ can be deduced from it.

We put $\epsilon_{i}:=\alpha_{i+1}^{\prime}-\alpha_{i}^{\prime}$ for $i=1, \ldots, l$. Take an $S$-ample line bundle $\mathcal{O}_{\mathcal{X}}(1)$ on $\mathcal{X}$. 
Proposition 5.2. $\quad$ There exists an integer $m_{0}$ such that for any geometric point $\left(E_{1}, E_{2}, \Phi, F_{*}\left(E_{1}\right)\right) \in \overline{\mathcal{M}_{\mathcal{X} / S}^{\mathcal{D}, \boldsymbol{\alpha}^{\prime}, \boldsymbol{\beta}, \gamma}}\left(r, d,\left\{d_{i}\right\}\right)(K)$, the inequality

$$
\begin{aligned}
& \frac{\beta_{1} \alpha_{1}^{\prime} h^{0}\left(E_{1}^{\prime}(m)\right)+\beta_{2} h^{0}\left(E_{2}^{\prime}(m-\gamma)\right)+\sum_{i=1}^{l} \beta_{1} \epsilon_{i} h^{0}\left(F_{i+1}\left(E_{1}^{\prime}\right)(m)\right)}{\beta_{1} \operatorname{rank}\left(E_{1}^{\prime}\right)+\beta_{2} \operatorname{rank}\left(E_{2}^{\prime}\right)} \\
& <\frac{\beta_{1} \alpha_{1}^{\prime} h^{0}\left(E_{1}(m)\right)+\beta_{2} h^{0}\left(E_{2}(m-\gamma)\right)+\sum_{i=1}^{l} \beta_{1} \epsilon_{i} h^{0}\left(F_{i+1}\left(E_{1}\right)(m)\right)}{\beta_{1} \operatorname{rank}\left(E_{1}\right)+\beta_{2} \operatorname{rank}\left(E_{2}\right)}
\end{aligned}
$$

holds for any proper non-zero parabolic $\Lambda_{\mathcal{D}_{K}}^{1}$-subtriple $\left(E_{1}^{\prime}, E_{2}^{\prime}, \Phi^{\prime}, F_{*}\left(E_{1}^{\prime}\right)\right)$ of $\left(E_{1}, E_{2}, \Phi, F_{*}\left(E_{1}\right)\right)$ and any integer $m \geq m_{0}$.

Proof. By Proposition 5.1, there exists an integer $N_{1}$ such that for any geometric point $\left(E_{1}, E_{2}, \Phi, F_{*}\left(E_{1}\right)\right)$ of $\overline{\mathcal{M}_{\mathcal{X} / S}^{\mathcal{D}, \boldsymbol{\alpha}^{\prime}, \boldsymbol{\beta}, \gamma}}\left(r, d,\left\{d_{i}\right\}\right), h^{i}\left(F_{j}\left(E_{1}\right)(m)\right)=$ $h^{i}\left(E_{2}(m-\gamma)\right)=0$ for $i>0,1 \leq j \leq l+1$ and $m \geq N_{1}$. There also exists an integer $e$ such that for any geometric point $\left(E_{1}, E_{2}, \Phi, F_{*}\left(E_{1}\right)\right)$ of $\overline{\mathcal{M}_{\mathcal{X} / S}^{\mathcal{D}, \boldsymbol{\alpha}^{\prime}, \boldsymbol{\beta}, \gamma}}\left(r, d,\left\{d_{i}\right\}\right)$ and for any coherent subsheaf $E^{\prime}$ of $E_{1}^{\oplus \beta_{1}} \oplus E_{2}^{\oplus \beta_{2}}(-\gamma)$, the inequality

$$
\operatorname{deg} E^{\prime} \leq \operatorname{rank} E^{\prime}\left(\mu\left(E_{1}^{\oplus \beta_{1}} \oplus E_{2}(-\gamma)^{\oplus \beta_{2}}\right)+e\right)
$$

holds. Note that we write $\mu(E):=\operatorname{rank}(E)^{-1} \operatorname{deg}(E)$ for a vector bundle $E$. Applying [MY], Lemma 2.6 to the case

$$
\begin{gathered}
P(m)=\frac{\beta_{1} \alpha_{1}^{\prime} \chi\left(E_{1}(m)\right)+\beta_{2} \chi\left(E_{2}(m-\gamma)\right)+\sum_{i=1}^{l} \beta_{1} \epsilon_{i} \chi\left(F_{i+1}\left(E_{1}\right)(m)\right)}{\beta_{1} \operatorname{rank} E_{1}+\beta_{2} \operatorname{rank} E_{2}}-1, \\
r=\operatorname{rank}\left(E_{1}^{\oplus \beta_{1}} \oplus E_{2}^{\oplus \beta_{2}}\right), \quad a=\mu\left(E_{1}^{\oplus \beta_{1}} \oplus E_{2}(-\gamma)^{\oplus \beta_{2}}\right)+e
\end{gathered}
$$

we can take integers $L, M$ such that $M \leq a$ and for any integer $m \geq L$, the inequality

$$
h^{0}\left(E^{\prime}(m)\right) \leq \operatorname{rank}\left(E^{\prime}\right) P(m)
$$

holds for any vector bundle $E^{\prime}$ on a fiber of $\mathcal{X}$ over $S$ satisfying $0<\operatorname{rank}\left(E^{\prime}\right)<$ $\beta_{1} \operatorname{rank}\left(E_{1}\right)+\beta_{2} \operatorname{rank}\left(E_{2}\right), \mu\left(E^{\prime}\right) \leq M$ and $\operatorname{deg} \tilde{E}^{\prime} \leq a \operatorname{rank}\left(\tilde{E}^{\prime}\right)$ for any proper nonzero coherent subsheaf $\tilde{E}^{\prime}$ of $E^{\prime}$.

Now we put

$$
\mathcal{G}:=\left\{\begin{array}{ll}
E^{\prime} & \begin{array}{l}
\text { there exists a geometric point }\left(E_{1}, E_{2}, \Phi, F_{*}\left(E_{1}\right)\right) \\
\text { of } \overline{\mathcal{M}_{\mathcal{X}}^{\mathcal{D}, \boldsymbol{\alpha}^{\prime}, \boldsymbol{\beta}, \gamma}}\left(r, d,\left\{d_{i}\right\}\right) \text { such that } E^{\prime} \text { is a subbundle of } E_{1}^{\oplus \beta_{1}} \oplus \\
E_{2}(-\gamma)^{\oplus \beta_{2}} \text { and } \mu\left(E^{\prime}\right) \geq M
\end{array}
\end{array}\right\} .
$$

Then $\mathcal{G}$ is bounded. Thus there exists an integer $L^{\prime} \geq L$ such that for any $E^{\prime} \in \mathcal{G}$ and any $m \geq L^{\prime}, E^{\prime}(m-\gamma)$ is generated by its global sections, $h^{i}\left(E^{\prime}(m-\right.$ 
$\gamma))=h^{i}\left(\left(F_{j}\left(E_{1}\right) \cap E^{\prime}\right)(m)\right)=0$ for $i>0$ and $1 \leq j \leq l+1$. If we put

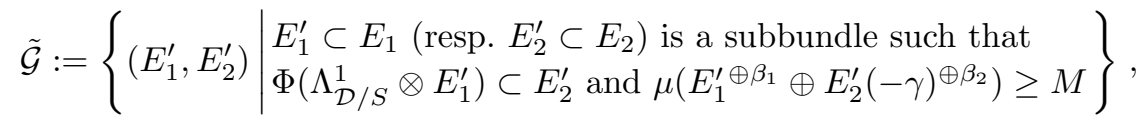

then the set of polynomials

$$
\begin{aligned}
& \left\{\beta_{1} \alpha_{1}^{\prime} \chi\left(E_{1}^{\prime}(m)\right)+\beta_{2} \chi\left(E_{2}^{\prime}(m-\gamma)\right)\right. \\
& \left.\quad+\sum_{i=1}^{l} \beta_{1} \epsilon_{i} \chi\left(\left(F_{i+1}\left(E_{1}\right) \cap E_{1}^{\prime}\right)(m)\right)\right\}_{\left(E_{1}^{\prime}, E_{2}^{\prime}\right) \in \tilde{\mathcal{G}}}
\end{aligned}
$$

is finite, because $E_{1}^{\prime \oplus \beta_{1}} \oplus E_{2}^{\prime}(-\tau)^{\oplus \beta_{2}} \in \mathcal{G}$ for any $\left(E_{1}^{\prime}, E_{2}^{\prime}\right) \in \tilde{\mathcal{G}}$. Thus there exists an integer $m_{0} \geq L^{\prime}$ such that for any $m \geq m_{0}$ and for any $\left(E_{1}^{\prime}, E_{2}^{\prime}\right) \in \tilde{\mathcal{G}}$, the inequality

$$
\begin{aligned}
\frac{\beta_{1} \alpha_{1}^{\prime} \chi\left(E_{1}^{\prime}(m)\right)+\beta_{2} \chi\left(E_{2}^{\prime}(m-\gamma)\right)+\sum_{i=1}^{l} \beta_{1} \epsilon_{i} \chi\left(\left(F_{i+1}\left(E_{1}\right) \cap E_{1}^{\prime}\right)(m)\right)}{\beta_{1} \operatorname{rank}\left(E_{1}^{\prime}\right)+\beta_{2} \operatorname{rank}\left(E_{2}^{\prime}\right)} & <P(m)+1
\end{aligned}
$$

holds. We can easily see that this $m_{0}$ satisfies the desired condition.

Proposition 5.3. Let $T$ be a noetherian scheme over $S$ and $\left(E_{1}, E_{2}, \Phi\right.$, $\left.F_{*}\left(E_{1}\right)\right)$ be a flat family of parabolic $\Lambda_{\mathcal{D}_{T} / T^{-}}^{1}$ triples on $\mathcal{X} \times_{S} T$ over $T$. Then there is an open subscheme $T^{s}$ of $T$ such that

$$
T^{s}(k)=\left\{t \in T(k) \mid\left(E_{1}, E_{2}, \Phi, F_{*}\left(E_{1}\right)\right) \otimes k(t) \text { is stable }\right\}
$$

for any algebraically closed field $k$.

Proof. We may assume that $T$ is connected. Put $P_{1}(m):=\chi\left(\left(E_{1} \otimes\right.\right.$ $k(s))(m)), P_{2}(m):=\chi\left(\left(E_{2} \otimes k(s)\right)(m-\gamma)\right)$ and $P_{1}^{(i)}(m):=\chi\left(\left(F_{i}\left(E_{1}\right) \otimes\right.\right.$ $k(s))(m))$ for a geometric point $s$ of $T$. Since the family $\mathcal{G}=\left\{E^{\prime} \mid \begin{array}{l}E^{\prime} \text { is a subbundle of }\left(E_{1}^{\oplus \beta_{1}} \oplus E_{2}(-\gamma)^{\oplus \beta_{2}}\right) \otimes k(s) \text { for some } \\ \text { geometric point } s \text { of } T \text { and } \mu\left(E^{\prime}\right) \geq \mu\left(\left(E_{1}, E_{2}, \Phi, F_{*}\left(E_{1}\right)\right) \otimes k(s)\right)\end{array}\right\}$ is bounded, the family

$$
\tilde{\mathcal{G}}=\left\{\begin{array}{l|l}
\left(E_{1}^{\prime}, E_{2}^{\prime}, \Phi^{\prime}, F_{*}\left(E_{1}^{\prime}\right)\right) & \begin{array}{l}
\left(E_{1}^{\prime}, E_{2}^{\prime}, \Phi^{\prime}, F_{*}\left(E_{1}^{\prime}\right)\right) \text { is a parabolic } \Lambda_{\mathcal{D}_{s}}^{1} \text {-subtriple } \\
\text { of }\left(E_{1}, E_{2}, \Phi, F_{*}\left(E_{1}\right)\right) \otimes k(s) \text { for some geometric } \\
\text { point } s \text { of } T \text { such that } E_{1}^{\prime} \subset E_{1} \otimes k(s) \text { (resp. } \\
\left.E_{2}^{\prime} \subset E_{2} \otimes k(s)\right) \text { is a subbundle and } \mu\left(E_{1}^{\prime}, E_{2}^{\prime},\right. \\
\left.\Phi^{\prime}, F_{*}\left(E_{1}^{\prime}\right)\right) \geq \mu\left(\left(E_{1}, E_{2}, \Phi, F_{*}\left(E_{1}\right)\right) \otimes k(s)\right)
\end{array}
\end{array}\right\}
$$


is also bounded. So the set of sequences of polynomials

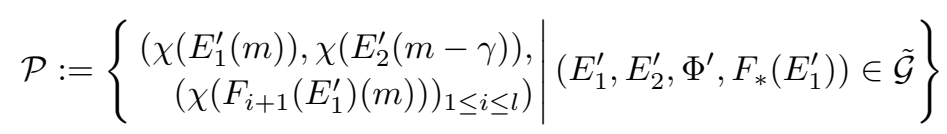

is finite. For each $\mathbf{P}^{\prime}:=\left(P_{1}^{\prime}, P_{2}^{\prime},\left(\left(P_{1}^{\prime}\right)^{(i+1)}\right)\right) \in \mathcal{P}$, put

$$
Q:=\operatorname{Quot}_{E_{1} / \mathcal{X}_{T} / T}^{P_{1}-P_{T}^{\prime}} \times_{T} \operatorname{Quot}_{E_{2} / \mathcal{X}_{T} / T}^{P_{2}-P_{2}^{\prime}}
$$

Let $\left(E_{1}\right)_{Q} \stackrel{\pi_{1}}{\rightarrow} G_{1}$ and $\left(E_{2}\right)_{Q} \stackrel{\pi_{2}}{\rightarrow} G_{2}$ be the universal quotient sheaves. We put

$$
Q^{\prime}:=\operatorname{Quot}_{\operatorname{ker} \pi_{1} / \mathcal{X}_{Q} / Q}^{P_{1}^{\prime}-\left(P^{\prime}(2)\right.} \times_{Q} \cdots \times_{Q} \operatorname{Quot}_{\operatorname{ker} \pi_{1} / \mathcal{X}_{Q} / Q}^{P_{1}^{\prime}-\left(P^{\prime}\right)^{(l+1)}} .
$$

Let $\left(\operatorname{ker} \pi_{1}\right)_{Q^{\prime}} \stackrel{\pi_{1}^{(i)}}{\rightarrow} G_{1}^{(i)}(1 \leq i \leq l)$ be the universal quotient sheaves. We consider the composite homomorphisms

$$
\begin{aligned}
& \Psi^{\prime}: \Lambda_{\mathcal{D} / S}^{1} \otimes\left(\operatorname{ker} \pi_{1}\right)_{Q^{\prime}} \hookrightarrow \Lambda_{\mathcal{D} / S}^{1} \otimes\left(E_{1}\right)_{Q^{\prime}} \stackrel{\Phi_{Q^{\prime}}}{\longrightarrow}\left(E_{2}\right)_{Q^{\prime}} \stackrel{\left(\pi_{2}\right)_{Q^{\prime}}}{\longrightarrow}\left(G_{2}\right)_{Q^{\prime}} \\
& \psi_{i}: \operatorname{ker} \pi_{1}^{(i+1)} \hookrightarrow\left(\operatorname{ker} \pi_{1}\right)_{Q^{\prime}} \stackrel{\pi_{1}^{(i)}}{\longrightarrow} G_{1}^{(i)} \quad(2 \leq i \leq l) \\
& \psi_{l+1}:\left(\operatorname{ker} \pi_{1}\right)_{Q^{\prime}} \otimes \mathcal{O}_{\mathcal{X}}(-\mathcal{D}) \longrightarrow\left(\operatorname{ker} \pi_{1}\right)_{Q^{\prime}} \stackrel{\pi_{1}^{(l+1)}}{\longrightarrow} G_{1}^{(l+1)} .
\end{aligned}
$$

Let $\tilde{Q}_{\mathbf{P}^{\prime}}^{\prime}$ be the maximal closed subscheme of $Q^{\prime}$ satisfying $\Psi_{\tilde{Q}_{\mathbf{P}^{\prime}}^{\prime}}^{\prime}=0$ and $\left(\psi_{i}\right)_{\tilde{Q}_{\mathbf{P}^{\prime}}^{\prime}}=0$ for $2 \leq i \leq l+1$. Since $f_{\mathbf{P}^{\prime}}: \tilde{Q}_{\mathbf{P}^{\prime}}^{\prime} \rightarrow T$ is a proper morphism,

$$
T^{s}=T \backslash \bigcup_{\mathbf{P}^{\prime} \in \mathcal{P}} f_{\mathbf{P}^{\prime}}\left(\tilde{Q}_{\mathbf{P}^{\prime}}^{\prime}\right)
$$

is an open subscheme which satisfies the desired condition.

\section{§5.3. Construction of the moduli space}

Now we construct the moduli scheme of $\overline{\mathcal{M}_{\mathcal{X} / S}^{\mathcal{D}, \boldsymbol{\alpha}^{\prime}, \boldsymbol{\beta}, \boldsymbol{\gamma}}}\left(r, d,\left\{d_{i}\right\}\right)$. We define a polynomial $P(m)$ in $m$ by $P(m):=r d_{\mathcal{X}} m+d+r(1-g)$ where $d_{\mathcal{X}}=\operatorname{deg} \mathcal{O}_{\mathcal{X}_{s}}(1)$ for $s \in S$ and $g$ is the genus of $\mathcal{X}_{s}$. We take an integer $m_{0}$ in Proposition 5.2. By Proposition 5.1, we may assume, by replacing $m_{0}$, that for any $m \geq m_{0}$, $h^{j}\left(F_{i}\left(E_{1}\right)(m)\right)=h^{j}\left(E_{2}(m-\gamma)\right)=0$ for $j>0, i=1, \ldots, l+1$ and $E_{2}(m-\gamma)$, $F_{i}\left(E_{1}\right)(m)(i=1, \ldots, l+1)$ are generated by their global sections for any geometric point $\left(E_{1}, E_{2}, \Phi, F_{*}\left(E_{1}\right)\right)$ of $\overline{\mathcal{M}_{\mathcal{X} / S}^{\mathcal{D}, \boldsymbol{\alpha}^{\prime}, \boldsymbol{\beta}, \gamma}}\left(r, d,\left\{d_{i}\right\}\right)$. Put $n_{1}=P\left(m_{0}\right)$ and $n_{2}=P\left(m_{0}-\gamma\right)$. Take two free $\mathcal{O}_{S}$-modules $V_{1}, V_{2}$ such that $\operatorname{rank} V_{1}=n_{1}$, 
$\operatorname{rank} V_{2}=n_{2}$. Let $Q_{1}$ be the Quot-scheme $\operatorname{Quot}_{V_{1} \otimes \mathcal{O}_{\mathcal{X}}\left(-m_{0}\right) / \mathcal{X} / S}^{P(m)}$ and $V_{1} \otimes$ $\mathcal{O}_{\mathcal{X}_{Q_{1}}}\left(-m_{0}\right) \rightarrow \mathcal{E}_{1}$ be the universal quotient sheaf. Similarly let $Q_{2}$ be the Quotscheme Quot ${ }_{V_{2} \otimes \mathcal{O}_{\mathcal{X}}\left(-m_{0}+\gamma\right) / \mathcal{X} / S}^{P(m)}$ and $V_{2} \otimes \mathcal{O}_{\mathcal{X}_{Q_{2}}}\left(-m_{0}+\gamma\right) \rightarrow \mathcal{E}_{2}$ be the universal quotient sheaf. We put $Q_{1}^{(i)}:=\operatorname{Quot}_{\mathcal{E}_{1} / \mathcal{X}_{Q_{1}} / Q_{1}}^{d_{i}}$. Let $F_{i+1}\left(\mathcal{E}_{1}\right) \subset\left(\mathcal{E}_{1}\right)_{Q_{1}^{(i)}}$ be the universal subsheaf. We define $Q$ as the maximal closed subscheme of $Q_{1}^{(1)} \times_{Q_{1}}$ $\cdots \times{ }_{Q_{1}} Q_{1}^{(l)} \times Q_{2}$ such that there are factorizations

$$
\left(\mathcal{E}_{1}\right)_{Q} \otimes \mathcal{O}_{\mathcal{X}_{Q}}\left(-\mathcal{D}_{Q}\right) \longrightarrow F_{i+1}\left(\mathcal{E}_{1}\right)_{Q} \hookrightarrow F_{i}\left(\mathcal{E}_{1}\right)_{Q} \subset\left(\mathcal{E}_{1}\right)_{Q}
$$

for $i=1, \ldots, l$, where $F_{1}\left(\mathcal{E}_{1}\right)=\mathcal{E}_{1}$. Since $\left(\mathcal{E}_{2}\right)_{Q}$ is flat over $Q$, there is a coherent sheaf $\mathcal{H}$ on $Q$ such that there is a functorial isomorphism

$$
\operatorname{Hom}_{\mathcal{X}_{T}}\left(\Lambda_{\mathcal{D} / S}^{1} \otimes_{\mathcal{O}_{\mathcal{X}}}\left(\mathcal{E}_{1}\right)_{T},\left(\mathcal{E}_{2}\right)_{T} \otimes \mathcal{L}\right) \cong \operatorname{Hom}_{T}\left(\mathcal{H} \otimes \mathcal{O}_{T}, \mathcal{L}\right)
$$

for any noetherian scheme $T$ over $Q$ and any quasi-coherent sheaf $\mathcal{L}$ on $T$.

We denote $\operatorname{Spec} S(\mathcal{H})$ by $\mathbf{V}^{*}(\mathcal{H})$, where $S(\mathcal{H})$ is the symmetric algebra of $\mathcal{H}$ over $\mathcal{O}_{Q}$. Let

$$
\tilde{\Phi}: \Lambda_{\mathcal{D} / S}^{1} \otimes_{\mathcal{O}_{\mathcal{X}}}\left(\mathcal{E}_{1}\right)_{\mathbf{V}^{*}(\mathcal{H})} \longrightarrow\left(\mathcal{E}_{2}\right)_{\mathbf{V}^{*}(\mathcal{H})}
$$

be the universal homomorphism. We define the open subscheme $R^{s}$ of $\mathbf{V}^{*}(\mathcal{H})$ by

$$
R^{s}:=\left\{\begin{array}{l|l}
s \in \mathbf{V}^{*}(\mathcal{H}) & \begin{array}{l}
\left(V_{1}\right)_{s} \rightarrow H^{0}\left(\left(\mathcal{E}_{1}\right)_{s}\left(m_{0}\right)\right),\left(V_{2}\right)_{s} \rightarrow H^{0}\left(\left(\mathcal{E}_{2}\right)_{s}\left(m_{0}-\gamma\right)\right) \\
\text { are bijective, } F_{i}\left(\mathcal{E}_{1}\right)_{s}\left(m_{0}\right),\left(\mathcal{E}_{2}\right)_{s}\left(m_{0}-\gamma\right) \text { are } \\
\text { generated by their global sections, } h^{j}\left(F_{i}\left(\mathcal{E}_{1}\right)_{s}\left(m_{0}\right)\right) \\
=h^{j}\left(\left(\mathcal{E}_{2}\right)_{s}\left(m_{0}-\gamma\right)\right)=0 \text { for } j>0,1 \leq i \leq l+1 \text { and } \\
\left(\left(\mathcal{E}_{1}\right)_{s},\left(\mathcal{E}_{2}\right)_{s}, \tilde{\Phi}_{s}, F_{*}\left(\mathcal{E}_{1}\right)_{s}\right) \text { is stable }
\end{array}
\end{array}\right\}
$$

For $y \in R^{s}$ and vector subspaces $V_{1}^{\prime} \subset\left(V_{1}\right)_{y}, V_{2}^{\prime} \subset\left(V_{2}\right)_{y}$, let $E\left(V_{1}^{\prime}, V_{2}^{\prime}, y\right)_{1}$ be the image of $V_{1}^{\prime} \otimes \mathcal{O}_{\mathcal{X}}\left(-m_{0}\right) \rightarrow\left(\mathcal{E}_{1}\right)_{y}$ and $E\left(V_{1}^{\prime}, V_{2}^{\prime}, y\right)_{2}$ be that of $V_{1}^{\prime} \otimes$ $\Lambda_{\mathcal{D}_{y}}^{1}\left(-m_{0}\right) \oplus V_{2}^{\prime} \otimes \mathcal{O}_{\mathcal{X}}\left(-m_{0}+\gamma\right) \rightarrow\left(\mathcal{E}_{2}\right)_{y}$. Since the family

$$
\mathcal{F}=\left\{\left(E\left(V_{1}^{\prime}, V_{2}^{\prime}, y\right)_{1}, E\left(V_{1}^{\prime}, V_{2}^{\prime}, y\right)_{2}\right) \mid y \in R^{s}, V_{1}^{\prime} \subset\left(V_{1}\right)_{y}, V_{2}^{\prime} \subset\left(V_{2}\right)_{y}\right\}
$$

is bounded, there exists an integer $m_{1}\left(\geq m_{0}\right)$ such that for all $m \geq m_{1}$,

$$
\begin{aligned}
& V_{1}^{\prime} \otimes H^{0}\left(\mathcal{O}_{\mathcal{X}_{y}}(m)\right) \rightarrow H^{0}\left(E\left(V_{1}^{\prime}, V_{2}^{\prime}, y\right)_{1}(m)\right) \\
& V_{1}^{\prime} \otimes H^{0}\left(\mathcal{O}_{\mathcal{X}_{y}}\left(m_{0}+m-\gamma\right) \otimes \Lambda_{\mathcal{D}_{y}}^{1} \otimes \mathcal{O}_{\mathcal{X}_{y}}\left(-m_{0}\right)\right) \\
& \quad \oplus V_{2}^{\prime} \otimes H^{0}\left(\mathcal{O}_{\mathcal{X}_{y}}(m)\right) \rightarrow H^{0}\left(E\left(V_{1}^{\prime}, V_{2}^{\prime}, y\right)_{2}(m-\gamma)\right)
\end{aligned}
$$


are surjective and $H^{i}\left(\mathcal{O}_{\mathcal{X}_{y}}\left(m_{0}+m-\gamma\right) \otimes \Lambda_{\mathcal{D}_{y}}^{1} \otimes \mathcal{O}_{\mathcal{X}_{y}}\left(-m_{0}\right)\right)=0, H^{i}\left(\mathcal{O}_{\mathcal{X}_{y}}(m)\right)=$ 0 for $i>0$ for all members $\left(E\left(V_{1}^{\prime}, V_{2}^{\prime}, y\right)_{1}, E\left(V_{1}^{\prime}, V_{2}^{\prime}, y\right)_{2}\right) \in \mathcal{F}$ and the inequality

$$
\begin{aligned}
& \left(\beta_{1} \operatorname{rank} E_{1}^{\prime}+\beta_{2} \operatorname{rank} E_{2}^{\prime}\right) d \mathcal{X} \\
& \quad \times\left(\beta_{1} h^{0}\left(\left(\mathcal{E}_{1}\right)_{y}\left(m_{0}\right)\right)+\beta_{2} h^{0}\left(\left(\mathcal{E}_{2}\right)_{y}\left(m_{0}-\gamma\right)\right)-\sum_{i=1}^{l} \beta_{1} \epsilon_{i} d_{i}\right) \\
& \quad-\left(\beta_{1}+\beta_{2}\right) r d_{\mathcal{X}}\left(\beta_{1} \alpha_{1}^{\prime} h^{0}\left(E_{1}^{\prime}\left(m_{0}\right)\right)+\beta_{2} h^{0}\left(E_{2}^{\prime}\left(m_{0}-\gamma\right)\right)\right. \\
& \left.\quad+\sum_{i=1}^{l} \beta_{1} \epsilon_{i} h^{0}\left(F_{i+1}\left(E_{1}^{\prime}\right)\left(m_{0}\right)\right)\right) \\
& >m^{-1}\left(\beta_{1} \operatorname{dim} V_{1}^{\prime}+\beta_{2} \operatorname{dim} V_{2}^{\prime}-\beta_{1} \chi\left(E_{1}^{\prime}\left(m_{0}\right)\right)-\beta_{2} \chi\left(E_{2}^{\prime}\left(m_{0}-\gamma\right)\right)\right) \\
& \quad \times\left(\beta_{1} \operatorname{dim} V_{1}+\beta_{2} \operatorname{dim} V_{2}-\sum_{i=1}^{l} \beta_{1} \epsilon_{i} d_{i}\right)
\end{aligned}
$$

holds for $(0,0) \subsetneq\left(V_{1}^{\prime}, V_{2}^{\prime}\right) \subsetneq\left(\left(V_{1}\right)_{y},\left(V_{2}\right)_{y}\right)$, where $E_{1}^{\prime}:=E\left(V_{1}^{\prime}, V_{2}^{\prime}, y\right)_{1}, E_{2}^{\prime}:=$ $E\left(V_{1}^{\prime}, V_{2}^{\prime}, y\right)_{2}$ and $F_{i+1}\left(E_{1}^{\prime}\right):=E_{1}^{\prime} \cap F_{i+1}\left(\mathcal{E}_{1}\right)_{y}$ for $i=1, \ldots, l$. From now on, we fix such a large integer $m_{1}$.

The composite

$$
V_{1} \otimes \Lambda_{\mathcal{D} / S}^{1} \otimes \mathcal{O}_{\mathcal{X}}\left(-m_{0}\right)_{R^{s}} \rightarrow \Lambda_{\mathcal{D} / S}^{1} \otimes\left(\mathcal{E}_{1}\right)_{R^{s}} \stackrel{\tilde{\Phi}}{\longrightarrow}\left(\mathcal{E}_{2}\right)_{R^{s}}
$$

induces a homomorphism

$$
V_{1} \otimes W_{1} \otimes \mathcal{O}_{R^{s}} \rightarrow\left(\pi_{R^{s}}\right)_{*}\left(\mathcal{E}_{2}\left(m_{0}+m_{1}-\gamma\right)_{R^{s}}\right),
$$

where $W_{1}:=\left(\pi_{S}\right)_{*}\left(\mathcal{O}_{\mathcal{X}}\left(m_{0}+m_{1}-\gamma\right) \otimes \Lambda_{\mathcal{D} / S}^{1} \otimes \mathcal{O}_{\mathcal{X}}\left(-m_{0}\right)\right)$ and the quotient $V_{2} \otimes \mathcal{O}_{\mathcal{X}}\left(-m_{0}+\gamma\right) \rightarrow \mathcal{E}_{2}$ induces a homomorphism

$$
V_{2} \otimes W_{2} \otimes \mathcal{O}_{R^{s}} \rightarrow\left(\pi_{R^{s}}\right)_{*}\left(\mathcal{E}_{2}\left(m_{0}+m_{1}-\gamma\right)_{R^{s}}\right),
$$

where $W_{2}:=\left(\pi_{S}\right)_{*}\left(\mathcal{O}_{\mathcal{X}}\left(m_{1}\right)\right)$. These homomorphisms induce a quotient bundle

$$
\left(V_{1} \otimes W_{1} \oplus V_{2} \otimes W_{2}\right) \otimes \mathcal{O}_{R^{s}} \longrightarrow\left(\pi_{R^{s}}\right)_{*}\left(\mathcal{E}_{2}\left(m_{0}+m_{1}-\gamma\right)_{R^{s}}\right) .
$$

This quotient and the canonical quotient bundles

$$
\begin{aligned}
& V_{1} \otimes W_{2} \otimes \mathcal{O}_{R^{s}}=V_{1} \otimes\left(\pi_{S}\right)_{*}\left(\mathcal{O}_{\mathcal{X}}\left(m_{1}\right)\right) \otimes \mathcal{O}_{R^{s}} \rightarrow\left(\pi_{R^{s}}\right)_{*}\left(\mathcal{E}_{1}\left(m_{0}+m_{1}\right)_{R^{s}}\right), \\
& V_{1} \otimes \mathcal{O}_{R^{s}} \rightarrow\left(\pi_{R^{s}}\right)_{*}\left(\mathcal{E}_{1} / F_{i+1}\left(\mathcal{E}_{1}\right)\left(m_{0}\right)_{R^{s}}\right) \quad(i=1, \ldots, l)
\end{aligned}
$$


determine a morphism

$$
\iota: R^{s} \rightarrow \operatorname{Grass}_{r_{2}}\left(V_{1} \otimes W_{1} \oplus V_{2} \otimes W_{2}\right) \times \operatorname{Grass}_{r_{1}}\left(V_{1} \otimes W_{2}\right) \times \prod_{i=1}^{l} \operatorname{Grass}_{d_{i}}\left(V_{1}\right)
$$

where $r_{1}=h^{0}\left(\mathcal{E}_{1}\left(m_{0}+m_{1}\right)_{s}\right), r_{2}:=h^{0}\left(\mathcal{E}_{2}\left(m_{0}+m_{1}-\gamma\right)_{s}\right)$ for any point $s \in$ $R^{s}$ and $\operatorname{Grass}_{r}(V)$ is the Grassmannian parametrizing $r$-dimensional quotient vector spaces of $V$. We can check that $\iota$ is an immersion.

We set $G:=\left(G L\left(V_{1}\right) \times G L\left(V_{2}\right)\right) /\left(\mathbf{G}_{m} \times S\right)$, where $\mathbf{G}_{m} \times S$ is contained in $G L\left(V_{1}\right) \times G L\left(V_{2}\right)$ as scalar matrices. Then $G$ acts canonically on $R^{s}$ and on $\operatorname{Grass}_{r_{2}}\left(V_{1} \otimes W_{1} \oplus V_{2} \otimes W_{2}\right) \times \operatorname{Grass}_{r_{1}}\left(V_{1} \otimes W_{2}\right) \times \prod_{i=1}^{l} \operatorname{Grass}_{d_{i}}\left(V_{1}\right)$. We can see that $\iota$ is a $G$-equivariant immersion. There is an $S$-ample line bundle $\mathcal{O}_{\operatorname{Grass}_{r_{2}}\left(V_{1} \otimes W_{1} \oplus V_{2} \otimes W_{2}\right)}(1)$ on $\operatorname{Grass}_{r_{2}}\left(V_{1} \otimes W_{1} \oplus V_{2} \otimes W_{2}\right)$ induced by Plücker embedding. Similarly there are canonical $S$-ample line bundles $\mathcal{O}_{\text {Grass }_{r_{1}}\left(V_{1} \otimes W_{2}\right)}$ $(1), \mathcal{O}_{\operatorname{Grass}_{d_{i}}\left(V_{1}\right)}(1)$, on $\operatorname{Grass}_{r_{1}}\left(V_{1} \otimes W_{2}\right), \operatorname{Grass}_{d_{i}}\left(V_{1}\right)$, respectively. We define positive rational numbers $\nu_{1}, \nu_{2}, \nu_{1}^{(i)}(1 \leq i \leq l)$ by

$$
\begin{aligned}
\nu_{1} & =\beta_{1}\left(\beta_{1} P\left(m_{0}\right)+\beta_{2} P\left(m_{0}-\gamma\right)-\sum_{i=1}^{l} \beta_{1} \epsilon_{i} d_{i}\right), \\
\nu_{2} & =\beta_{2}\left(\beta_{1} P\left(m_{0}\right)+\beta_{2} P\left(m_{0}-\gamma\right)-\sum_{i=1}^{l} \beta_{1} \epsilon_{i} d_{i}\right), \\
\nu_{1}^{(i)} & =\left(\beta_{1}+\beta_{2}\right) \beta_{1} r d_{\mathcal{X}} m_{1} \epsilon_{i} .
\end{aligned}
$$

Let us consider the $\mathbf{Q}$-line bundle

$$
\begin{aligned}
L:=\iota^{*}\left(\mathcal{O}_{\operatorname{Grass}_{r_{2}}\left(V_{1} \otimes W_{1} \oplus V_{2} \otimes W_{2}\right)}\left(\nu_{1}\right) \otimes \mathcal{O}_{\operatorname{Grass}_{r_{1}}\left(V_{1} \otimes W_{2}\right)}\left(\nu_{2}\right) \otimes\right. \\
\\
\left.\bigotimes_{i=1}^{l} \mathcal{O}_{\operatorname{Grass}_{d_{i}}\left(V_{1}\right)}\left(\nu_{1}^{(i)}\right)\right)
\end{aligned}
$$

on $R^{s}$. Then for some positive integer $N, L^{\otimes N}$ becomes a $G$-linearized $S$-ample line bundle on $R^{s}$.

Proposition 5.4. $\quad$ All points of $R^{s}$ are properly stable with respect to the action of $G$ and the $G$-linearized $S$-ample line bundle $L^{\otimes N}$.

Proof. Take any geometric point $x$ of $R^{s}$. Let $y$ be the induced geometric point of $S$. We must show that $x$ is a properly stable point of the fiber $R_{y}^{s}$ with 
respect to the action of $G_{y}$ and the polarization $L_{y}^{\otimes N}$. So we may assume that $S=\operatorname{Spec} K$ with $K$ an algebraically closed field. We put

$$
\left(E_{1}, E_{2}, \Phi, F_{*}\left(E_{1}\right)\right):=\left(\left(\mathcal{E}_{1}\right)_{x},\left(\mathcal{E}_{2}\right)_{x}, \tilde{\Phi}_{x}, F_{*}\left(\mathcal{E}_{1}\right)_{x}\right) .
$$

Let

$$
\begin{array}{r}
\pi_{2}: V_{1} \otimes W_{1} \oplus V_{2} \otimes W_{2} \rightarrow N_{2}, \quad \pi_{1}: V_{1} \otimes W_{2} \rightarrow N_{1}, \quad \pi_{1}^{(i)}: V_{1} \rightarrow N_{1}^{(i)} \\
(i=1, \ldots, l)
\end{array}
$$

be the quotient vector spaces corresponding to $\iota(x)$. We will show that $\iota(x)$ is a properly stable point with respect to the action of $G$ and the linearization of $L^{\otimes N}$. Consider the character

$$
\chi: G L\left(V_{1}\right) \times G L\left(V_{2}\right) \longrightarrow \mathbf{G}_{m} ; \quad\left(g_{1}, g_{2}\right) \mapsto \operatorname{det}\left(g_{1}\right)^{\beta_{1}} \operatorname{det}\left(g_{2}\right)^{\beta_{2}}
$$

Then there is an isogeny $\operatorname{ker} \chi \longrightarrow G$ and we may prove the stability with respect to the action of ker $\chi$ instead of $G$. Take any one parameter subgroup $\lambda$ of ker $\chi$. For a suitable basis $e_{1}^{(1)}, \ldots, e_{n_{1}}^{(1)}$ (resp. $\left.e_{1}^{(2)}, \ldots, e_{n_{2}}^{(2)}\right)$ of $V_{1}$ (resp. $V_{2}$ ), the action of $\lambda$ on $V_{1}$ (resp. $V_{2}$ ) is represented by

$$
e_{i}^{(1)} \mapsto t^{u_{i}^{(1)}} e_{i}^{(1)}\left(\operatorname{resp} . e_{i}^{(2)} \mapsto t^{u_{i}^{(2)}} e_{i}^{(2)}\right) \quad\left(t \in \mathbf{G}_{m}\right)
$$

where $u_{1}^{(1)} \leq \cdots \leq u_{n_{1}}^{(1)}\left(\right.$ resp. $\left.u_{1}^{(2)} \leq \cdots \leq u_{n_{2}}^{(2)}\right)$ and $\sum_{i=1}^{n_{1}} \beta_{1} u_{i}^{(1)}+\sum_{i=1}^{n_{2}} \beta_{2} u_{i}^{(2)}$ $=0$. Take a basis $f_{1}^{(k)}, \ldots, f_{b_{k}}^{(k)}$ of $W_{k}$ for $k=1,2$.

We define functions $a_{1}(p)$ and $a_{2}(p)$ in $p \in\left\{0,1, \ldots, \beta_{1} n_{1}+\beta_{2} n_{2}\right\}$ as follows. First we put $\left(a_{1}(0), a_{2}(0)\right):=(0,0)$. We put

$$
\left(a_{1}(1), a_{2}(1)\right):= \begin{cases}(1,0) & \text { if } \beta_{1} u_{1}^{(1)} \leq \beta_{2} u_{1}^{(2)} \\ (0,1) & \text { if } \beta_{1} u_{1}^{(1)}>\beta_{2} u_{2}^{(2)}\end{cases}
$$

Inductively we define 


$$
\begin{cases}\left(a_{1}(p+1), a_{2}(p+1)\right):=\left(a_{1}(p), a_{2}(p)\right) & \text { if } p<\beta_{1} a_{1}(p)+\beta_{2} a_{2}(p) \\ \left(a_{1}(p+1), a_{2}(p+1)\right):=\left(a_{1}(p)+1, a_{2}(p)\right) & \text { if } p=\beta_{1} a_{1}(p)+\beta_{2} a_{2}(p) \\ & \beta_{1} u_{a_{1}(p)+1}^{(1)} \leq \beta_{2} u_{a_{2}(p)+1}^{(2)} \\ & \text { and } a_{1}(p)<n_{1} \\ \left(a_{1}(p+1), a_{2}(p+1)\right):=\left(a_{1}(p), a_{2}(p)+1\right) & \text { if } p=\beta_{1} a_{1}(p)+\beta_{2} a_{2}(p) \\ & \beta_{1} u_{a_{1}(p)+1}^{(1)}>\beta_{2} u_{a_{2}(p)+1}^{(2)} \\ & \text { and } a_{2}(p)<n_{2} . \\ \left(a_{1}(p+1), a_{2}(p+1)\right):=\left(a_{1}(p)+1, a_{2}(p)\right) & \text { if } p=\beta_{1} a_{1}(p)+\beta_{2} a_{2}(p) \\ & \text { and } a_{2}(p)=n_{2} \\ \left(a_{1}(p+1), a_{2}(p+1)\right):=\left(a_{1}(p), a_{2}(p)+1\right) & \text { if } p=\beta_{1} a_{1}(p)+\beta_{2} a_{2}(p) \\ & \text { and } a_{1}(p)=n_{1}\end{cases}
$$

Then $a_{1}(p)$ and $a_{2}(p)$ are integers with $0 \leq a_{1}(p) \leq n_{1}, 0 \leq a_{2}(p) \leq n_{2}$, $a_{1}(p) \leq a_{1}(p+1)$ and $a_{2}(p) \leq a_{2}(p+1)$. We define $v_{1}, \ldots, v_{\beta_{1} n_{1}+\beta_{2} n_{2}}$ and $e_{1}^{\prime}, \ldots, e_{\beta_{1} n_{1}+\beta_{2} n_{2}}^{\prime}$ by

$$
\begin{cases}v_{p}:=\beta_{1} u_{a_{1}(p)}^{(1)}, e_{p}^{\prime}:=e_{a_{1}(p)}^{(1)} & \text { if } a_{1}(p-1)<a_{1}(p) \\ v_{p}:=\beta_{2} u_{a_{2}(p)}^{(2)}, e_{p}^{\prime}:=e_{a_{2}(p)}^{(2)} & \text { if } a_{2}(p-1)<a_{2}(p) \\ v_{p}:=v_{p-1}, e_{p}^{\prime}:=e_{p-1}^{\prime} & \text { if } a_{1}(p-1)=a_{1}(p) \text { and } a_{2}(p-1)=a_{2}(p) .\end{cases}
$$

We put $\delta_{p}:=\left(v_{p+1}-v_{p}\right)\left(\beta_{1} n_{1}+\beta_{2} n_{2}\right)^{-1}$ for $p=1, \ldots, \beta_{1} n_{1}+\beta_{2} n_{2}-1$. Then $\delta_{p}$ are non-negative rational numbers and for each $1 \leq i \leq n_{1}$

$$
\beta_{1} u_{i}^{(1)}=\sum_{\substack{1 \leq p \leq \beta_{1} n_{1}+\beta_{2} n_{2}-1 \\ a_{1}(p)<i}} p \delta_{p}+\sum_{\substack{1 \leq p \leq \beta_{1} n_{1}+\beta_{2} n_{2}-1 \\ a_{1}(p) \geq i}}\left(p-\beta_{1} n_{1}-\beta_{2} n_{2}\right) \delta_{p}
$$

and for each $1 \leq i \leq n_{2}$

$$
\beta_{2} u_{i}^{(2)}=\sum_{\substack{1 \leq p \leq \beta_{1} n_{1}+\beta_{2} n_{2}-1 \\ a_{2}(p)<i}} p \delta_{p}+\sum_{\substack{1 \leq p \leq \beta_{1} n_{1}+\beta_{2} n_{2}-1 \\ a_{2}(p) \geq i}}\left(p-\beta_{1} n_{1}-\beta_{2} n_{2}\right) \delta_{p} .
$$

For $\mu=1, \ldots, \beta_{1} n_{1} b_{1}+\beta_{2} n_{2} b_{2}$, we can find unique integers $p_{0}, p_{1} \in$ $\left\{0,1, \ldots, \beta_{1} n_{1}+\beta_{2} n_{2}\right\}$ such that

$$
\begin{gathered}
\left(a_{1}\left(p_{1}\right), a_{2}\left(p_{1}\right)\right)=\left(a_{1}\left(p_{0}+1\right), a_{2}\left(p_{0}+1\right)\right)=\left(a_{1}\left(p_{0}\right)+1, a_{2}\left(p_{0}\right)\right), \text { or } \\
\left(a_{1}\left(p_{1}\right), a_{2}\left(p_{1}\right)\right)=\left(a_{1}\left(p_{0}+1\right), a_{2}\left(p_{0}+1\right)=\left(a_{1}\left(p_{0}\right), a_{2}\left(p_{0}\right)+1\right)\right.
\end{gathered}
$$


and

$$
\mu= \begin{cases} & \text { for some } 1 \leq j \leq b_{1} \\ \left(a_{1}\left(p_{0}\right) \beta_{1}+\left(p_{1}-p_{0}-1\right)\right) b_{1}+a_{2}\left(p_{0}\right) \beta_{2} b_{2}+j & \text { if }\left(a_{1}\left(p_{1}\right), a_{2}\left(p_{1}\right)\right) \\ & =\left(a_{1}\left(p_{0}\right)+1, a_{2}\left(p_{0}\right)\right) \\ & \text { for some } 1 \leq j \leq b_{2} \\ a_{1}\left(p_{0}\right) \beta_{1} b_{1}+\left(a_{2}\left(p_{0}\right) \beta_{2}+\left(p_{1}-p_{0}-1\right)\right) b_{2}+j & \text { if }\left(a_{1}\left(p_{1}\right), a_{2}\left(p_{1}\right)\right) \\ & =\left(a_{1}\left(p_{0}\right), a_{2}\left(p_{0}\right)+1\right) .\end{cases}
$$

For such $\mu$, we put $s_{\mu}^{(2)}:=v_{p_{1}}$ and

$$
h_{\mu}^{\prime}:= \begin{cases}e_{p_{1}}^{\prime} \otimes f_{j}^{(1)} & \text { if }\left(a_{1}\left(p_{1}\right), a_{2}\left(p_{1}\right)\right)=\left(a_{1}\left(p_{0}\right)+1, a_{2}\left(p_{0}\right)\right) \\ e_{p_{1}}^{\prime} \otimes f_{j}^{(2)} & \text { if }\left(a_{1}\left(p_{1}\right), a_{2}\left(p_{1}\right)\right)=\left(a_{1}\left(p_{0}\right), a_{2}\left(p_{0}\right)+1\right) .\end{cases}
$$

Let $U_{\mu}^{(2)}$ be the vector subspace of $V_{1} \otimes W_{1} \oplus V_{2} \otimes W_{2}$ generated by $h_{1}^{\prime}, \ldots, h_{\mu}^{\prime}$. We put $U_{0}^{(2)}=0$. For $q=1, \ldots, r_{2}$, we can find an integer $\mu_{q}^{(2)} \in\left\{1, \ldots, \beta_{1} n_{1} b_{1}+\right.$ $\left.\beta_{2} n_{2} b_{2}\right\}$ such that $\operatorname{dim} \pi_{2}\left(U_{\mu_{q}^{(2)}}^{(2)}\right)=q$ and $\operatorname{dim} \pi_{2}\left(U_{\mu_{q}^{(2)}-1}^{(2)}\right)=q-1$. Then

$$
\begin{aligned}
\sum_{q=1}^{r_{2}} s_{\mu_{q}^{(2)}}^{(2)} & =\sum_{q=1}^{r_{2}} s_{\mu_{q}^{(2)}}^{(2)}\left(\operatorname{dim} \pi_{2}\left(U_{\mu_{q}^{(2)}}^{(2)}\right)-\operatorname{dim} \pi_{2}\left(U_{\mu_{q}^{(2)}-1}^{(2)}\right)\right) \\
& =\sum_{\mu=1}^{\beta_{1} n_{1} b_{1}+\beta_{2} n_{2} b_{2}} s_{\mu}^{(2)}\left(\operatorname{dim} \pi_{2}\left(U_{\mu}^{(2)}\right)-\operatorname{dim} \pi_{2}\left(U_{\mu-1}^{(2)}\right)\right) \\
& =r_{2} s_{\beta_{1} n_{1} b_{1}+\beta_{2} n_{2} b_{2}}^{(2)}-\sum_{\mu=1}^{\beta_{1} n_{1} b_{1}+\beta_{2} n_{2} b_{2}-1}\left(s_{\mu+1}^{(2)}-s_{\mu}^{(2)}\right) \operatorname{dim} \pi_{2}\left(U_{\mu}^{(2)}\right) \\
& =r_{2} v_{\beta_{1} n_{1}+\beta_{2} n_{2}}-\sum_{p=1}^{\beta_{1} n_{1}+\beta_{2} n_{2}-1}\left(v_{p+1}-v_{p}\right) \operatorname{dim} \pi_{2}\left(U_{\beta_{1} a_{1}(p) b_{1}+\beta_{2} a_{2}(p) b_{2}}^{(2)}\right) \\
& =\sum_{p=1}^{\beta_{1} n_{1}+\beta_{2} n_{2}-1}\left(r_{2} p-\left(\beta_{1} n_{1}+\beta_{2} n_{2}\right) \operatorname{dim} \pi_{2}\left(U_{\beta_{1} a_{1}(p) b_{1}+\beta_{2} a_{2}(p) b_{2}}^{(2)}\right)\right) \delta_{p} .
\end{aligned}
$$

For $\mu=(i-1) b_{2}+j$, put $h_{\mu}^{(1)}:=e_{i}^{(1)} \otimes f_{j}^{(2)}$ for $i=1, \ldots, n_{1}, j=1, \ldots, b_{2}$. We define integers $s_{1}^{(1)}, \ldots, s_{b_{2} n_{1}}^{(1)}$ by putting $s_{\mu}^{(1)}:=\beta_{1} u_{i}^{(1)}$ for $\mu=(i-1) b_{2}+j$ with $1 \leq j \leq b_{2}$. Let $U_{\mu}^{(1)}$ be the vector subspace of $V_{1} \otimes W_{2}$ generated by $h_{1}^{(1)}, \ldots, h_{\mu}^{(1)}$ for $\mu=1, \ldots, b_{2} n_{1}$. We put $U_{0}^{(1)}=0$. For $q=1, \ldots, r_{1}$, let $\mu_{q}^{(1)}$ be the integer such that $\operatorname{dim} \pi_{1}\left(U_{\mu_{q}^{(1)}}^{(1)}\right)=q$ and $\operatorname{dim} \pi_{1}\left(U_{\mu_{q}^{(1)}-1}^{(1)}\right)=q-1$. Then

$$
\sum_{q=1}^{r_{1}} s_{\mu_{q}^{(1)}}^{(1)}=\sum_{\mu=1}^{b_{2} n_{1}} s_{\mu}^{(1)}\left(\operatorname{dim} \pi_{1}\left(U_{\mu}^{(1)}\right)-\operatorname{dim} \pi_{1}\left(U_{\mu-1}^{(1)}\right)\right)
$$




$$
\begin{aligned}
& =r_{1} s_{b_{2} n_{1}}^{(1)}-\sum_{\mu=1}^{b_{2} n_{1}-1}\left(s_{\mu+1}^{(1)}-s_{\mu}^{(1)}\right) \operatorname{dim} \pi_{1}\left(U_{\mu}^{(1)}\right) \\
& =r_{1} \beta_{1} u_{n_{1}}^{(1)}-\sum_{i=1}^{n_{1}-1}\left(u_{i+1}^{(1)}-u_{i}^{(1)}\right) \beta_{1} \operatorname{dim} \pi_{1}\left(U_{i b_{2}}^{(1)}\right) \\
& =r_{1} \beta_{1} u_{n_{1}}^{(1)}+\sum_{a_{1}(p)<n_{1}}\left(v_{p+1}-v_{p}\right) \operatorname{dim} \pi_{1}\left(U_{a_{1}(p) b_{2}}^{(1)}\right) \\
& =r_{1}\left(\sum_{\substack{1 \leq p \leq \beta_{1} n_{1}+\beta_{2} n_{2}-1 \\
a_{1}(p)<n_{1}}} p \delta_{p}+\sum_{\substack{1 \leq p \leq \beta_{1} n_{1}+\beta_{2} n_{2}-1 \\
a_{1}(p) \geq n_{1}}}\left(p-\beta_{1} n_{1}-\beta_{2} n_{2}\right) \delta_{p}\right) \\
& -\sum_{\substack{1 \leq p \leq \beta_{1} n_{1}+\beta_{2} n_{2}-1 \\
a_{1}(p)<n_{1}}}\left(\beta_{1} n_{1}+\beta_{2} n_{2}\right) \delta_{p} \operatorname{dim} \pi_{1}\left(U_{a_{1}(p) b_{2}}^{(1)}\right) \\
& =\sum_{p=1}^{\beta_{1} n_{1}+\beta_{2} n_{2}-1}\left(r_{1} p-\left(\beta_{1} n_{1}+\beta_{2} n_{2}\right) \operatorname{dim} \pi_{1}\left(U_{a_{1}(p) b_{2}}^{(1)}\right)\right) \delta_{p}
\end{aligned}
$$

Let $V_{p}^{(1)}$ be the vector subspace of $V_{1}$ generated by $e_{1}^{(1)}, \ldots, e_{p}^{(1)}$. We put $V_{0}^{(1)}=0$. For $i=1, \ldots, l$ and for $q=1, \ldots, d_{i}$, let $\mu_{q}^{i}$ be the integer such that $\operatorname{dim} \pi_{1}^{(i)}\left(V_{\mu_{q}^{i}}^{(1)}\right)=q$ and $\operatorname{dim} \pi_{1}^{(i)}\left(V_{\mu_{q}^{i}-1}^{(1)}\right)=q-1$. Then

$$
\begin{aligned}
\sum_{q=1}^{d_{i}} \beta_{1} u_{\mu_{q}^{i}}^{(1)}= & \sum_{q=1}^{d_{i}} \beta_{1} u_{\mu_{q}^{i}}^{(1)}\left(\operatorname{dim} \pi_{1}^{(i)}\left(V_{\mu_{q}^{i}}^{(1)}\right)-\operatorname{dim} \pi_{1}^{(i)}\left(V_{\mu_{q}^{i}-1}^{(1)}\right)\right) \\
= & \sum_{p=1}^{n_{1}} \beta_{1} u_{p}^{(1)}\left(\operatorname{dim} \pi_{1}^{(i)}\left(V_{p}^{(1)}\right)-\operatorname{dim} \pi_{1}^{(i)}\left(V_{p-1}^{(1)}\right)\right) \\
= & d_{i} \beta_{1} u_{n_{1}}^{(1)}-\sum_{p=1}^{n_{1}-1} \beta_{1}\left(u_{p+1}^{(1)}-u_{p}^{(1)}\right) \operatorname{dim} \pi_{1}^{(i)}\left(V_{p}^{(1)}\right) \\
= & d_{i} \beta_{1} u_{n_{1}}^{(1)}-\sum_{a_{1}(p)<n_{1}}\left(v_{p+1}-v_{p}\right) \operatorname{dim} \pi_{1}^{(i)}\left(V_{a_{1}(p)}^{(1)}\right) \\
= & \left.d_{i} \sum_{\substack{1 \leq p \leq \beta_{1} n_{1}+\beta_{2} n_{2}-1 \\
a_{1}(p)<n_{1}}}^{\substack{1 \leq p \leq \beta_{1} n_{1}+\beta_{2} n_{2}-1 \\
a_{1}(p)<n_{1}}} \sum_{\substack{1 \leq \beta_{1} n_{1}+\beta_{2} n_{2}-1 \\
a_{1}(p) \geq n_{1}}}\left(p-\beta_{1} n_{1}-\beta_{2} n_{2}\right) \delta_{p}\right) \\
& \left.-\beta_{1} n_{1}+\beta_{2} n_{2}\right) \delta_{p} \operatorname{dim} \pi_{1}^{(i)}\left(V_{a_{1}(p)}^{(1)}\right)
\end{aligned}
$$




$$
=\sum_{p=1}^{\beta_{1} n_{1}+\beta_{2} n_{2}-1}\left(d_{i} p-\left(\beta_{1} n_{1}+\beta_{2} n_{2}\right) \operatorname{dim} \pi_{1}^{(i)}\left(V_{a_{1}(p)}^{(1)}\right)\right) \delta_{p} .
$$

Thus we have

$$
\begin{aligned}
& \mu^{L^{\otimes N}}(x, \lambda) \\
&=-\left(\sum_{k=1}^{2} \nu_{k} \sum_{q=1}^{r_{k}} s_{\mu_{q}^{(k)}}^{(k)}+\sum_{i=1}^{l} \nu_{1}^{(i)} \sum_{q=1}^{d_{i}} \beta_{1} u_{\mu_{q}^{i}}^{(1)}\right) N \\
&=\sum_{p=1}^{\beta_{1} n_{1}+\beta_{2} n_{2}-1} N \delta_{p}\left\{\left(-p \sum_{i=1}^{l} \nu_{1}^{(i)} d_{i}+\left(\beta_{1} n_{1}+\beta_{2} n_{2}\right) \sum_{i=1}^{l} \nu_{1}^{(i)} \operatorname{dim} \pi_{1}^{(i)}\left(V_{a_{1}(p)}^{(1)}\right)\right)\right. \\
&-\left(\nu_{1} r_{1}+\nu_{2} r_{2}\right) p+\left(\beta_{1} n_{1}+\beta_{2} n_{2}\right) \\
&\left.\times\left(\nu_{1} \operatorname{dim} \pi_{1}\left(U_{a_{1}(p) b_{2}}^{(1)}\right)+\nu_{2} \operatorname{dim} \pi_{2}\left(U_{\beta_{1} a_{1}(p) b_{1}+\beta_{2} a_{2}(p) b_{2}}^{(2)}\right)\right)\right\} .
\end{aligned}
$$

See [Mum], Definition 2.2 for the definition of $\mu^{L^{\otimes N}}(x, \lambda)$. By [Mum], Theorem $2.1, x$ is a properly stable point if

$$
\begin{aligned}
- & p\left(\nu_{1} r_{1}+\nu_{2} r_{2}\right)+\left(\beta_{1} n_{1}+\beta_{2} n_{2}\right)\left(\nu_{1} \operatorname{dim} \pi_{1}\left(U_{a_{1}(p) b_{2}}^{(1)}\right)\right. \\
& \left.+\nu_{2} \operatorname{dim} \pi_{2}\left(U_{\beta_{1} a_{1}(p) b_{1}+\beta_{2} a_{2}(p) b_{2}}^{(2)}\right)\right) \\
& -p \sum_{i=1}^{l} \nu_{1}^{(i)} d_{i}+\left(\beta_{1} n_{1}+\beta_{2} n_{2}\right) \sum_{i=1}^{l} \nu_{1}^{(i)} \operatorname{dim} \pi_{1}^{(i)}\left(V_{a_{1}(p)}^{(1)}\right)>0
\end{aligned}
$$

for all $p=1, \ldots, \beta_{1} n_{1}+\beta_{2} n_{2}-1$.

For each $p\left(1 \leq p \leq \beta_{1} n_{1}+\beta_{2} n_{2}-1\right)$, let $V_{k}^{\prime}$ be the vector subspace of $V_{k}$ generated by $e_{1}^{(\bar{k})}, \ldots, e_{a_{k}(p)}^{(k)}$ for $k=1,2$. Then $U_{a_{1}(p) b_{2}}^{(1)}=V_{1}^{\prime} \otimes W_{2}$ and $U_{\beta_{1} a_{1}(p) b_{1}+\beta_{2} a_{2}(p) b_{2}}^{(2)}=V_{1}^{\prime} \otimes W_{1} \oplus V_{2}^{\prime} \otimes W_{2}$. Put

$$
\begin{aligned}
& E_{1}^{\prime}:=\operatorname{Im}\left(V_{1}^{\prime} \otimes \mathcal{O}_{\mathcal{X}}\left(-m_{0}\right) \rightarrow E_{1}\right), F_{i+1}\left(E_{1}^{\prime}\right):=F_{i+1}\left(E_{1}\right) \cap E_{1}^{\prime},(i=1, \ldots, l), \\
& E_{2}^{\prime}:=\operatorname{Im}\left(V_{1}^{\prime} \otimes \Lambda_{\mathcal{D} / S}^{1}\left(-m_{0}\right) \oplus V_{2}^{\prime} \otimes \mathcal{O}_{\mathcal{X}}\left(-m_{0}+\gamma\right) \rightarrow E_{2}\right), \Phi^{\prime}:=\left.\Phi\right|_{\Lambda_{\mathcal{D} / S}^{1}} \otimes E_{1}^{\prime}
\end{aligned}
$$

Then $\left(E_{1}^{\prime}, E_{2}^{\prime}, \Phi^{\prime}, F_{*}\left(E_{1}^{\prime}\right)\right)$ is a parabolic $\Lambda_{\mathcal{D}_{K}}^{1}$-subtriple of $\left(E_{1}, E_{2}, \Phi, F_{*}\left(E_{1}\right)\right)$. By the choice of $m_{1}$, we have $\pi_{2}\left(U_{\beta_{1} a_{1}(p) b_{1}+\beta_{2} a_{2}(p) b_{2}}^{(2)}\right)=H^{0}\left(E_{2}^{\prime}\left(m_{0}+m_{1}-\gamma\right)\right)$ and $\pi_{1}\left(U_{a_{1}(p) b_{2}}^{(1)}\right)=H^{0}\left(E_{1}^{\prime}\left(m_{0}+m_{1}\right)\right)$. Put $r_{1}^{\prime}:=\operatorname{rank} E_{1}^{\prime}, r_{2}^{\prime}:=\operatorname{rank} E_{2}^{\prime}$. Let $V_{1}^{\prime(i)}$ be the kernel of the composite $V_{1}^{\prime} \hookrightarrow V_{1} \stackrel{\pi_{1}^{(i)}}{\longrightarrow} N_{1}^{(i)}$. Then we have $-p\left(\nu_{1} r_{1}+\nu_{2} r_{2}\right)+\left(\beta_{1} n_{1}+\beta_{2} n_{2}\right)\left(\nu_{1} \operatorname{dim} \pi_{1}\left(U_{a_{1}(p) b_{2}}^{(1)}\right)\right.$ 


$$
\begin{aligned}
& \left.+\nu_{2} \operatorname{dim} \pi_{2}\left(U_{\beta_{1} a_{1}(p) b_{1}+\beta_{2} a_{2}(p) b_{2}}^{(2)}\right)\right) \\
& -p \sum_{i=1}^{l} \nu_{1}^{(i)} d_{i}+\left(\beta_{1} n_{1}+\beta_{2} n_{2}\right) \sum_{i=1}^{l} \nu_{1}^{(i)} \operatorname{dim} \pi_{1}^{(i)}\left(V_{a_{1}(p)}^{(1)}\right) \\
& \geq\left(\beta_{1} \operatorname{dim} V_{1}+\beta_{2} \operatorname{dim} V_{2}-\sum_{i=1}^{l} \beta_{1} \epsilon_{i} d_{i}\right) \\
& \times\left\{-\left(\beta_{1} \operatorname{dim} V_{1}^{\prime}+\beta_{2} \operatorname{dim} V_{2}^{\prime}\right)\left(\beta_{1} h^{0}\left(E_{1}\left(m_{0}+m_{1}\right)\right)+\beta_{2} h^{0}\left(E_{2}\left(m_{0}+m_{1}-\gamma\right)\right)\right)\right. \\
& \left.+\left(\beta_{1} \operatorname{dim} V_{1}+\beta_{2} \operatorname{dim} V_{2}\right)\left(\beta_{1} h^{0}\left(E_{1}^{\prime}\left(m_{0}+m_{1}\right)\right)+\beta_{2} h^{0}\left(E_{2}^{\prime}\left(m_{0}+m_{1}-\gamma\right)\right)\right)\right\} \\
& -\left(\beta_{1} \operatorname{dim} V_{1}^{\prime}+\beta_{2} \operatorname{dim} V_{2}^{\prime}\right) \sum_{i=1}^{l} \nu_{1}^{(i)} d_{i} \\
& +\left(\beta_{1} \operatorname{dim} V_{1}+\beta_{2} \operatorname{dim} V_{2}\right) \sum_{i=1}^{l} \nu_{1}^{(i)}\left(\operatorname{dim} V_{1}^{\prime}-\operatorname{dim} V_{1}^{\prime(i)}\right) \\
& =\left(\beta_{1} \operatorname{dim} V_{1}+\beta_{2} \operatorname{dim} V_{2}-\sum_{i=1}^{l} \beta_{1} \epsilon_{i} d_{i}\right) \\
& \times\left\{-\left(\beta_{1} \operatorname{dim} V_{1}^{\prime}+\beta_{2} \operatorname{dim} V_{2}^{\prime}\right)\left(r d_{\mathcal{X}}\left(\beta_{1}+\beta_{2}\right) m_{1}+\beta_{1} \operatorname{dim} V_{1}+\beta_{2} \operatorname{dim} V_{2}\right)\right. \\
& +\left(\beta_{1} \operatorname{dim} V_{1}+\beta_{2} \operatorname{dim} V_{2}\right)\left(\left(\beta_{1} r_{1}^{\prime}+\beta_{2} r_{2}^{\prime}\right) d_{\mathcal{X}} m_{1}\right. \\
& \left.\left.+\beta_{1} \chi\left(E_{1}^{\prime}\left(m_{0}\right)\right)+\beta_{2} \chi\left(E_{2}^{\prime}\left(m_{0}-\gamma\right)\right)\right)\right\} \\
& -\left(\beta_{1} \operatorname{dim} V_{1}^{\prime}+\beta_{2} \operatorname{dim} V_{2}^{\prime}\right) \sum_{i=1}^{l} \nu_{1}^{(i)} d_{i} \\
& +\left(\beta_{1} \operatorname{dim} V_{1}+\beta_{2} \operatorname{dim} V_{2}\right) \sum_{i=1}^{l} \nu_{1}^{(i)}\left(\operatorname{dim} V_{1}^{\prime}-\operatorname{dim} V_{1}^{\prime(i)}\right) \\
& =\left(\beta_{1} \operatorname{dim} V_{1}+\beta_{2} \operatorname{dim} V_{2}-\sum_{i=1}^{l} \beta_{1} \epsilon_{i} d_{i}\right) \\
& \times\left\{-r d_{\mathcal{X}}\left(\beta_{1}+\beta_{2}\right) m_{1}\left(\beta_{1} \operatorname{dim} V_{1}^{\prime}+\beta_{2} \operatorname{dim} V_{2}^{\prime}\right)\right. \\
& \left.+\left(\beta_{1} r_{1}^{\prime}+\beta_{2} r_{2}^{\prime}\right) d_{\mathcal{X}} m_{1}\left(\beta_{1} \operatorname{dim} V_{1}+\beta_{2} \operatorname{dim} V_{2}\right)\right\} \\
& +\left(\beta_{1} \operatorname{dim} V_{1}+\beta_{2} \operatorname{dim} V_{2}-\sum_{i=1}^{l} \beta_{1} \epsilon_{i} d_{i}\right)\left(\beta_{1} \operatorname{dim} V_{1}+\beta_{2} \operatorname{dim} V_{2}\right) \\
& \times\left\{-\left(\beta_{1} \operatorname{dim} V_{1}^{\prime}+\beta_{2} \operatorname{dim} V_{2}^{\prime}\right)+\left(\beta_{1} \chi\left(E_{1}^{\prime}\left(m_{0}\right)\right)+\beta_{2} \chi\left(E_{2}^{\prime}\left(m_{0}-\gamma\right)\right)\right)\right\}
\end{aligned}
$$




$$
\begin{aligned}
& -\left(\beta_{1} \operatorname{dim} V_{1}^{\prime}+\beta_{2} \operatorname{dim} V_{2}^{\prime}\right) \sum_{i=1}^{l}\left(\beta_{1}+\beta_{2}\right) \beta_{1} r d_{\mathcal{X}} m_{1} \epsilon_{i} d_{i} \\
& +\left(\beta_{1} \operatorname{dim} V_{1}+\beta_{2} \operatorname{dim} V_{2}\right) \sum_{i=1}^{l}\left(\beta_{1}+\beta_{2}\right) \beta_{1} r d_{\mathcal{X}} m_{1} \epsilon_{i}\left(\operatorname{dim} V_{1}^{\prime}-\operatorname{dim} V_{1}^{\prime(i)}\right) \\
= & -\left(\beta_{1} \operatorname{dim} V_{1}+\beta_{2} \operatorname{dim} V_{2}\right)\left(\beta_{1}+\beta_{2}\right) r d_{\mathcal{X}} m_{1} \\
& \times\left(\beta_{1} \operatorname{dim} V_{1}^{\prime}+\beta_{2} \operatorname{dim} V_{2}^{\prime}-\sum_{i=1}^{l} \beta_{1} \epsilon_{i}\left(\operatorname{dim} V_{1}^{\prime}-\operatorname{dim} V_{1}^{\prime(i)}\right)\right) \\
& +\left(\beta_{1} \operatorname{dim} V_{1}+\beta_{2} \operatorname{dim} V_{2}\right)\left(\beta_{1} r_{1}^{\prime}+\beta_{2} r_{2}^{\prime}\right) d_{\mathcal{X}} m_{1} \\
& \times\left(\beta_{1} \operatorname{dim} V_{1}+\beta_{2} \operatorname{dim} V_{2}-\sum_{i=1}^{l} \beta_{1} \epsilon_{i} d_{i}\right) \\
& +\left(\beta_{1} \operatorname{dim} V_{1}+\beta_{2} \operatorname{dim} V_{2}-\sum_{i=1}^{l} \beta_{1} \epsilon_{i} d_{i}\right)\left(\beta_{1} \operatorname{dim} V_{1}+\beta_{2} \operatorname{dim} V_{2}\right) \\
& \times\left(-\left(\beta_{1} \operatorname{dim} V_{1}^{\prime}+\beta_{2} \operatorname{dim} V_{2}^{\prime}\right)+\left(\beta_{1} \chi\left(E_{1}^{\prime}\left(m_{0}\right)\right)+\beta_{2} \chi\left(E_{2}^{\prime}\left(m_{0}-\gamma\right)\right)\right)\right. \\
\geq & \left(\beta_{1} \operatorname{dim} V_{1}+\beta_{2} \operatorname{dim} V_{2}\right) \\
& \times\left(\beta_{1} \operatorname{dim} V_{1}+\beta_{2} \operatorname{dim} V_{2}-\sum_{i=1}^{l} \beta_{1} \epsilon_{i} d_{i}\right)\left(\beta_{1} \operatorname{dim} V_{1}+\beta_{2} \operatorname{dim} V_{2}\right) \\
& \times\left\{\left(\beta_{1} r_{1}^{\prime}+\beta_{2} r_{2}^{\prime}\right) d \mathcal{X} m_{1}\left(\beta_{1} h^{0}\left(E_{1}\left(m_{0}\right)\right)+\beta_{2} h^{0}\left(E_{2}\left(m_{0}-\gamma\right)\right)-\sum_{i=1}^{l} \beta_{1} \epsilon_{i} d_{i}\right)\right. \\
& -\left(\beta_{1}+\beta_{2}\right) r d_{\mathcal{X}} m_{1}\left(\beta_{1} h^{0}\left(E_{1}^{\prime}\left(m_{0}\right)\right)+\beta_{2} h^{0}\left(E_{2}^{\prime}\left(m_{0}-\gamma\right)\right)\right. \\
& \left.-\sum_{i=1}^{l} \beta_{1} \epsilon_{i}\left(h^{0}\left(E_{1}^{\prime}\left(m_{0}\right)\right)-h^{0}\left(F_{i+1}\left(E_{1}^{\prime}\right)\left(m_{0}\right)\right)\right)\right\} \\
& \left.\left.+\beta_{2} \operatorname{dim} V_{2}^{\prime}\right)+\left(\beta_{1} \chi\left(E_{1}^{\prime}\left(m_{0}\right)\right)+\beta_{2} \chi\left(E_{2}^{\prime}\left(m_{0}-\gamma\right)\right)\right)\right) \\
& \left.+V_{1}\right) \\
& \\
& \\
&
\end{aligned}
$$

Note that the last inequality holds by the choice of $m_{1}$. Hence $x$ is a properly stable point.

By Proposition 5.4, there exists a geometric quotient $R^{s} / G$. The following proposition follows from a standard argument.

Theorem 5.1. $\overline{M_{\mathcal{X} / S}^{\mathcal{D}, \boldsymbol{\alpha}^{\prime}, \boldsymbol{\beta}, \gamma}}\left(r, d,\left\{d_{i}\right\}\right):=R^{s} / G$ is a coarse moduli scheme of $\overline{\mathcal{M}_{\mathcal{X} / S}^{\mathcal{D}, \boldsymbol{\alpha}^{\prime}, \boldsymbol{\beta}, \gamma}}\left(r, d,\left\{d_{i}\right\}\right)$. 
Remark 5.2. The quotient map $R^{s} \rightarrow \overline{M_{\mathcal{X} / S}^{\mathcal{D}, \boldsymbol{\alpha}^{\prime}, \boldsymbol{\beta}, \gamma}}\left(r, d,\left\{d_{i}\right\}\right)$ is a principal $G$-bundle, which we can see by the following lemma and the same argument as [M], Proposition 6.4.

Lemma 5.1. Take any geometric point $\left(E_{1}, E_{2}, \Phi, F_{*}\left(E_{1}\right)\right) \quad \in$ $\overline{M_{\mathcal{X} / S}^{\mathcal{D}, \boldsymbol{\alpha}^{\prime}, \boldsymbol{\beta}, \gamma}\left(r, d,\left\{d_{i}\right\}\right)}(K)$. Then for any endomorphisms $f_{1}: E_{1} \rightarrow E_{1}, f_{2}$ : $E_{2} \rightarrow E_{2}$ satisfying $\Phi \circ\left(1 \otimes f_{1}\right)=f_{2} \circ \Phi$ and $f_{1}\left(F_{i+1}\left(E_{1}\right)\right) \subset F_{i+1}\left(E_{1}\right)$ for $1 \leq i \leq l$, there exists $c \in K$ such that $\left(f_{1}, f_{2}\right)=\left(c \cdot \operatorname{id}_{E_{1}}, c \cdot \operatorname{id}_{E_{2}}\right)$.

Proof. Take such $\left(f_{1}, f_{2}\right)$. Let $c \in K$ be an eigenvalue of $f_{1} \otimes k(x)$ for some $x \in \mathcal{X}_{K}(K)$. Then $f_{1}-c \cdot \mathrm{id}_{E_{1}}$ becomes an endomorphism of $E_{1}$ which is not an isomorphism. Put $E_{1}^{\prime}:=\operatorname{Im}\left(f_{1}-c \cdot \operatorname{id}_{E_{1}}\right), E_{2}^{\prime}:=\operatorname{Im}\left(f_{2}-c \cdot \operatorname{id}_{E_{2}}\right)$, $\Phi^{\prime}:=\left.\Phi\right|_{\Lambda_{\mathcal{D}_{K}}^{1} \otimes E_{1}^{\prime}}$ and $F_{i+1}\left(E_{1}^{\prime}\right):=\left(f_{1}-c \cdot \operatorname{id}_{E_{1}}\right)\left(F_{i+1}\left(E_{1}\right)\right)$ for $i=1, \ldots, l$. Then $\left(E_{1}^{\prime}, E_{2}^{\prime}, \Phi^{\prime}, F_{*}\left(E_{1}^{\prime}\right)\right)$ becomes a parabolic $\Lambda_{\mathcal{D}_{K}}^{1}$-subtriple of $\left(E_{1}, E_{2}, \Phi, F_{*}\left(E_{1}\right)\right)$. If we put $G_{1}:=\operatorname{ker}\left(E_{1} \rightarrow E_{1}^{\prime}\right), G_{2}:=\operatorname{ker}\left(E_{2} \rightarrow E_{2}^{\prime}\right), \Phi_{G}:=\left.\Phi\right|_{\Lambda_{\mathcal{D}_{K}}^{1} \otimes G_{1}}$ and $F_{i+1}\left(G_{1}\right):=F_{i+1}\left(E_{1}\right) \cap G_{1}$ for $i=1, \ldots, l$, then $\left(G_{1}, G_{2}, \Phi_{G}, F_{*}\left(G_{1}\right)\right)$ becomes a parabolic $\Lambda_{\mathcal{D}_{K}}^{1}$-subtriple of $\left(E_{1}, E_{2}, \Phi, F_{*}\left(E_{1}\right)\right)$. If $\left(E_{1}^{\prime}, E_{2}^{\prime}\right) \neq(0,0)$, then, by the stability of $\left(E_{1}, E_{2}, \Phi, F_{*}\left(E_{1}\right)\right)$, we must have the inequalities

$$
\begin{array}{r}
\frac{\beta_{1} \alpha_{1}^{\prime} \chi\left(E_{1}(m)\right)+\beta_{2} \chi\left(E_{2}(m-\gamma)\right)+\sum_{i=1}^{l} \beta_{1} \epsilon_{i} \chi\left(F_{i+1}\left(E_{1}\right)(m)\right)}{\beta_{1} \operatorname{rank}\left(E_{1}\right)+\beta_{2} \operatorname{rank}\left(E_{2}\right)} \\
>\frac{\beta_{1} \alpha_{1}^{\prime} \chi\left(E_{1}^{\prime}(m)\right)+\beta_{2} \chi\left(E_{2}^{\prime}(m-\gamma)\right)+\sum_{i=1}^{l} \beta_{1} \epsilon_{i} \chi\left(F_{i+1}\left(E_{1}^{\prime}\right)(m)\right)}{\beta_{1} \operatorname{rank}\left(E_{1}^{\prime}\right)+\beta_{2} \operatorname{rank}\left(E_{2}^{\prime}\right)} \\
>\frac{\beta_{1} \alpha_{1}^{\prime} \chi\left(E_{1}(m)\right)+\beta_{2} \chi\left(E_{2}(m-\gamma)\right)+\sum_{i=1}^{l} \beta_{1} \epsilon_{i} \chi\left(F_{i+1}\left(E_{1}\right)(m)\right)}{\beta_{1} \operatorname{rank}\left(E_{1}\right)+\beta_{2} \operatorname{rank}\left(E_{2}\right)}
\end{array}
$$

for $m \gg 0$, which is a contradiction. Therefore we have $\left(E_{1}^{\prime}, E_{2}^{\prime}\right)=(0,0)$, which means that $\left(f_{1}, f_{2}\right)=\left(c \cdot \operatorname{id}_{E_{1}}, c \cdot \operatorname{id}_{E_{2}}\right)$.

\section{§5.4. Projectivity of the moduli space}

Proposition 5.5. $\quad$ Let $R$ be a discrete valuation ring over $S$ with residue field $k=R / m$ and quotient field $K$. Let $\left(E_{1}, E_{2}, \Phi, F_{*}\left(E_{1}\right)\right)$ be a semistable parabolic $\Lambda_{\mathcal{D}_{K}}^{1}$-triple on $\mathcal{X}_{K}$. Then there exists a flat family $\left(\tilde{E}_{1}, \tilde{E}_{2}, \tilde{\Phi}_{,}, F_{*}\left(\tilde{E}_{1}\right)\right)$ of parabolic $\Lambda_{\mathcal{D}_{R}}^{1}$-triples on $\mathcal{X}_{R}$ over $R$ such that $\left(E_{1}, E_{2}, \Phi, F_{*}\left(E_{1}\right)\right) \cong$ $\left(\tilde{E}_{1}, \tilde{E}_{2}, \tilde{\Phi}, F_{*}\left(\tilde{E}_{1}\right)\right) \otimes_{R} K$ and that $\left(\tilde{E}_{1}, \tilde{E}_{2}, \tilde{\Phi}, F_{*}\left(\tilde{E}_{1}\right)\right) \otimes_{R} k$ is semistable.

Proof. Two surjections

$$
V_{1} \otimes \mathcal{O}_{\mathcal{X}_{K}}\left(-m_{0}\right) \cong H^{0}\left(E_{1}\left(m_{0}\right)\right) \otimes \mathcal{O}_{\mathcal{X}_{K}}\left(-m_{0}\right) \rightarrow E_{1},
$$




$$
V_{2} \otimes \mathcal{O}_{\mathcal{X}_{K}}\left(-m_{0}+\gamma\right) \cong H^{0}\left(E_{2}\left(m_{0}-\gamma\right)\right) \otimes \mathcal{O}_{\mathcal{X}_{K}}\left(-m_{0}+\gamma\right) \rightarrow E_{2}
$$

and the quotients $E_{1} \rightarrow E_{1} / F_{i+1}\left(E_{1}\right)(i=1, \ldots, l)$ give a morphism $f:$ Spec $K \rightarrow Q$, where $Q$ is defined by the property (105) in subsection 5.3. Since $Q$ is proper over $S, f$ extends to a morphism $\tilde{f}:$ Spec $R \rightarrow Q$. Thus there are coherent sheaves $E_{1}^{(0)}, E_{2}^{(0)}$ on $\mathcal{X}_{R}$ flat over $R$ and a flat family of filtrations $F_{*}\left(E_{1}^{(0)}\right)$ of $E_{1}^{(0)}$ such that $E_{1}^{(0)} \otimes K \cong E_{1}, E_{2}^{(0)} \otimes K \cong E_{2}$ and $F_{*}\left(E_{1}^{(0)}\right) \otimes_{R} K=$ $F_{*}\left(E_{1}\right)$. The pullback of $\mathcal{H}$ by the morphism $\tilde{f}$ : Spec $R \rightarrow Q$ is denoted by $\mathcal{H}_{R}$. Recall that $\mathcal{H}$ is defined by (106) in subsection 5.3. The homomorphism $\Phi: \Lambda_{\mathcal{D} / S}^{1} \otimes E_{1} \rightarrow E_{2}$ corresponds to a homomorphism $\psi: \mathcal{H}_{R} \otimes_{R} K \rightarrow K$. There is a non-zero element $t \in K \backslash\{0\}$ and a homomorphism $\tilde{\psi}: \mathcal{H}_{R} \rightarrow R$ such that $t \psi=\tilde{\psi} \otimes_{R} K$. Let $\Phi^{(0)}: \Lambda_{\mathcal{D} / S}^{1} \otimes E_{1}^{(0)} \rightarrow E_{2}^{(0)}$ be the homomorphism corresponding to $\tilde{\psi}$. Then we have $\left(E_{1}, E_{2}, \Phi, F_{*}\left(E_{1}\right)\right) \cong\left(E_{1}^{(0)}, E_{2}^{(0)}, \Phi^{(0)}, F_{*}\left(E_{1}^{(0)}\right)\right) \otimes_{R} K$, since $\left(E_{1}, E_{2}, \Phi, F_{*}\left(E_{1}\right)\right) \cong\left(E_{1}, E_{2}, t \Phi, F_{*}\left(E_{1}\right)\right)$. Our proposition follows from the following claim:

Claim. There is a flat family $\left(\tilde{E}_{1}, \tilde{E}_{2}, \tilde{\Phi}, F_{*}\left(\tilde{E}_{1}\right)\right)$ of parabolic $\Lambda_{\mathcal{D}_{R}}^{1}$ triples on $\mathcal{X}_{R}$ over $R$ such that $\tilde{E}_{j} \subset E_{j}^{(0)}$ for $j=1,2, F_{i+1}\left(\tilde{E}_{1}\right) \subset F_{i+1}\left(E_{1}^{(0)}\right)$ for $i=1, \ldots, l, \tilde{\Phi}=\left.\Phi^{(0)}\right|_{\tilde{E}_{1} \otimes \Lambda_{\mathcal{D} / S}^{1}},\left(\tilde{E}_{1}, \tilde{E}_{2}, \tilde{\Phi}, F_{*}\left(\tilde{E}_{1}\right)\right) \otimes_{R} K \cong\left(E_{1}, E_{2}, \Phi, F_{*}\left(E_{1}\right)\right)$ and $\left(\tilde{E}_{1}, \tilde{E}_{2}, \tilde{\Phi}, F_{*}\left(\tilde{E}_{1}\right)\right) \otimes_{R} k$ is semistable.

Assume that $E_{1}^{(0)} \otimes k$ or $E_{2}^{(0)} \otimes k$ have torsions. In this case let $B_{1}^{(0)}$ and $B_{2}^{(0)}$ be the torsion parts of $E_{1}^{(0)} \otimes k$ and $E_{2}^{(0)} \otimes k$, respectively. Then there are exact sequences

$$
\begin{aligned}
& 0 \rightarrow B_{1}^{(0)} \longrightarrow E_{1}^{(0)} \otimes k \longrightarrow G_{1}^{(0)} \rightarrow 0 \\
& 0 \rightarrow B_{2}^{(0)} \longrightarrow E_{2}^{(0)} \otimes k \longrightarrow G_{2}^{(0)} \rightarrow 0
\end{aligned}
$$

where $G_{1}^{(0)}$ and $G_{2}^{(0)}$ are vector bundles on $\mathcal{X}_{k}$. Put $E_{1}^{(1)}:=\operatorname{ker}\left(E_{1}^{(0)} \rightarrow\left(\left(E_{1}^{(0)} \otimes\right.\right.\right.$ $\left.\left.k) / B_{1}^{(0)}\right)\right), E_{2}^{(1)}:=\operatorname{ker}\left(E_{2}^{(0)} \rightarrow\left(\left(E_{2}^{(0)} \otimes k\right) / B_{2}^{(0)}\right)\right), \Phi^{(1)}:=\left.\Phi^{(0)}\right|_{\Lambda_{\mathcal{D}_{R}}^{1} \otimes E_{1}^{(1)}}$ and $F_{i+1}\left(E_{1}^{(1)}\right):=F_{i+1}\left(E_{1}^{(0)}\right) \cap E_{1}^{(1)}$ for $i=1, \ldots, l$. Then there are exact sequences

$$
\begin{aligned}
& 0 \rightarrow G_{1}^{(0)} \longrightarrow E_{1}^{(1)} \otimes k \longrightarrow B_{1}^{(0)} \rightarrow 0 \\
& 0 \rightarrow G_{2}^{(0)} \longrightarrow E_{2}^{(1)} \otimes k \longrightarrow B_{2}^{(0)} \rightarrow 0 .
\end{aligned}
$$

Again let $B_{1}^{(1)}$ and $B_{2}^{(1)}$ be the torsion parts of $E_{1}^{(1)} \otimes k$ and $E_{2}^{(1)} \otimes k$, respectively. Repeating these operations, we obtain sequences $\left(E_{1}^{(n)}, E_{2}^{(n)}, \Phi^{(n)}\right.$, $\left.F_{*}\left(E_{1}^{(n)}\right)\right)_{n \geq 0}, \quad\left(B_{1}^{(n)}, B_{2}^{(n)}\right)_{n \geq 0}$ and $\left(G_{1}^{(n)}, G_{2}^{(n)}\right)_{n \geq 0}$. Then the injections $B_{1}^{(n+1)} \hookrightarrow B_{1}^{(n)}, B_{2}^{(n+1)} \hookrightarrow B_{2}^{(n)}$ are induced by the homomorphisms $E_{1}^{(n+1)} \otimes$ 
$k \rightarrow E_{1}^{(n)} \otimes k, E_{2}^{(n+1)} \otimes k \rightarrow E_{2}^{(n)} \otimes k$. Since (length $B_{1}^{(n)}$, length $\left.B_{2}^{(n)}\right)_{n \geq 0}$ is stationary, we may assume that it is constant. Then we have isomorphisms $B_{1}^{(n+1)} \stackrel{\sim}{\rightarrow} B_{1}^{(n)}, B_{2}^{(n+1)} \stackrel{\sim}{\rightarrow} B_{2}^{(n)}, G_{1}^{(n)} \stackrel{\sim}{\rightarrow} G_{1}^{(n+1)}, G_{2}^{(n)} \stackrel{\sim}{\rightarrow} G_{2}^{(n+1)}$ for all $n$. Assume that $\left(B_{1}^{(n)}, B_{2}^{(n)}\right) \neq(0,0)$. There is an exact sequence

$$
E_{j}^{(n)} / m^{n} E_{j}^{(0)} \stackrel{u}{\longrightarrow} E_{j}^{(0)} / m^{n} E_{j}^{(0)} \longrightarrow E_{j}^{(0)} / E_{j}^{(n)} \rightarrow 0
$$

for $n \geq 1$ and $j=1,2$. We can see that $\left(E_{j}^{(n)} / m^{n} E_{j}^{(0)}\right) \otimes k \cong B_{j}^{(n-1)}$ and that

$$
u \otimes k:\left(E_{j}^{(n)} / m^{n} E_{j}^{(0)}\right) \otimes k \cong B_{j}^{(n-1)} \rightarrow E_{j}^{(0)} \otimes k
$$

is injective. Thus $E_{j}^{(0)} / E_{j}^{(n)}$ is flat over $R / m^{n}$ and the quotient $E_{j}^{(0)} / m^{n} E_{j}^{(0)} \rightarrow$ $E_{j}^{(0)} / E_{j}^{(n)}$ determines a morphism $f_{n}: \operatorname{Spec} R / m^{n} \rightarrow \operatorname{Quot}_{E_{j}^{(0)} / \mathcal{X}_{R} / R}$ for $n \geq$ 1. So we obtain a morphism $f: \operatorname{Spec} \hat{R} \rightarrow \operatorname{Quot}_{E_{j}^{(0)} / \mathcal{X}_{R} / R}$, where $\hat{R}$ is the completion of $R$. $f$ corresponds to a quotient sheaf $E_{j}^{(0)} \otimes \hat{R} \stackrel{\pi}{\rightarrow} G$. Since $(\operatorname{ker} \pi) \otimes R / m \cong B_{j}^{(0)}, \operatorname{ker} \pi \otimes \hat{K}$ is a torsion submodule of $E_{j}^{(0)}$, which is nonzero either for $j=1$ or $j=2$, where $\hat{K}$ is the quotient field of $\hat{R}$. However, it is a contradiction, because $E_{1}^{(0)} \otimes \hat{K}, E_{2}^{(0)} \otimes \hat{K}$ are vector bundles. Hence we must have $\left(B_{1}^{(n)}, B_{2}^{(n)}\right)=(0,0)$ for some $n$. So we may assume without loss of generality that $E_{1}^{(0)} \otimes k$ and $E_{2}^{(0)} \otimes k$ are locally free.

Now assume that the claim does not hold. Then we can define a descending sequence of flat families of parabolic $\Lambda_{\mathcal{D}_{R}}^{1}$-triples

$$
\begin{aligned}
\left(E_{1}^{(0)}, E_{2}^{(0)}, \Phi^{(0)}, F_{*}\left(E_{1}^{(0)}\right)\right) \supset\left(E_{1}^{(1)}, E_{2}^{(1)}\right. & \left., \Phi^{(1)}, F_{*}\left(E_{1}^{(1)}\right)\right) \\
& \supset\left(E_{1}^{(2)}, E_{2}^{(2)}, \Phi^{(2)}, F_{*}\left(E_{1}^{(2)}\right)\right) \supset \cdots
\end{aligned}
$$

as follows: Suppose $\left(E_{1}^{(n)}, E_{2}^{(n)}, \Phi^{(n)}, F_{*}\left(E_{1}^{(n)}\right)\right)$ has already been defined. There exists a maximal destabilizer $\left(B_{1}^{(n)}, B_{2}^{(n)}, \Phi_{B^{(n)}}, F_{*}\left(B_{1}^{(n)}\right)\right)$ of $\left(E_{1}^{(n)}, E_{2}^{(n)}\right.$, $\left.\Phi^{(n)}, F_{*}\left(E_{1}^{(n)}\right)\right) \otimes k$ as in the usual case of semistability of coherent sheaves. We can see that $B_{j}^{(n)}$ is a subbundle of $E_{j}^{(n)} \otimes k$ for $j=1,2$ and $F_{i+1}\left(B_{1}^{(n)}\right)=B_{1}^{(n)} \cap$ $\left(F_{i+1}\left(E_{1}^{(n)}\right) \otimes k\right)$ for $i=1, \ldots, l$. We put $G_{j}^{(n)}:=\left(E_{j}^{(n)} \otimes k\right) / B_{j}^{(n)}$ for $j=1,2$. Then $G_{1}^{(n)}$ has an induced quotient parabolic structure $F_{*}\left(G_{1}^{(n)}\right)$. A homomorphism $\Phi_{G^{(n)}}: \Lambda_{\mathcal{D} / S}^{1} \otimes G_{1}^{(n)} \rightarrow G_{2}^{(n)}$ is induced by $\Phi^{(n)}$ and $\left(G_{1}^{(n)}, G_{2}^{(n)}, \Phi_{G^{(n)}}\right.$, $\left.F_{*}\left(G_{1}^{(n)}\right)\right)$ becomes a parabolic $\Lambda_{\mathcal{D}_{k}}^{1}$-triple. Put

$$
\begin{gathered}
E_{j}^{(n+1)}=\operatorname{ker}\left(E_{j}^{(n)} \rightarrow G_{j}^{(n)}\right), \quad \Phi^{(n+1)}:=\left.\Phi^{(n)}\right|_{\Lambda_{\mathcal{D} / S}^{1} \otimes E_{1}^{(n+1)}}, \\
F_{i+1}\left(E_{1}^{(n+1)}\right)=\operatorname{ker}\left(F_{i+1}\left(E_{1}^{(n)}\right) \rightarrow F_{i+1}\left(G_{1}^{(n)}\right)\right)
\end{gathered}
$$


Then $\left(E_{1}^{(n+1)}, E_{2}^{(n+1)}, \Phi^{(n+1)}, F_{*}\left(E_{1}^{(n+1)}\right)\right)$ becomes a flat family of parabolic $\Lambda_{\mathcal{D}_{R}}^{1}$-triples on $\mathcal{X}_{R}$ over $R$. There are exact sequences

$0 \rightarrow B_{j}^{(n)} \rightarrow E_{j}^{(n)} \otimes k \rightarrow G_{j}^{(n)} \rightarrow 0 \quad$ and $\quad 0 \rightarrow G_{j}^{(n)} \rightarrow E_{j}^{(n+1)} \otimes k \rightarrow B_{j}^{(n)} \rightarrow 0$

for $j=1,2$. Then we can see that $\left(G_{1}^{(n)}, G_{2}^{(n)}, \Phi_{G^{(n)}}, F_{*}\left(G_{1}^{(n)}\right)\right)$ becomes a parabolic $\Lambda_{\mathcal{D}_{k}}^{1}$-subtriple of $\left(E_{1}^{(n+1)}, E_{2}^{(n+1)}, \Phi^{(n+1)}, F_{*}\left(E_{1}^{(n+1)}\right)\right) \otimes k$. We can check that $F_{i+1}\left(G_{1}^{(n)}\right)=G_{1}^{(n)} \cap\left(F_{i+1}\left(E_{1}^{(n+1)}\right) \otimes k\right)$ for $i=1, \ldots, l$. Put

$$
\begin{gathered}
C_{j}^{(n)}:=G_{j}^{(n)} \cap B_{j}^{(n+1)}, \quad \Phi_{C(n)}:=\left.\left(\Phi^{(n+1)} \otimes k\right)\right|_{\Lambda_{\mathcal{D}_{k}}^{1} \otimes C_{1}^{(n)}}, \\
F_{i+1}\left(C_{1}^{(n)}\right):=F_{i+1}\left(G_{1}^{(n)}\right) \cap F_{i+1}\left(B_{1}^{(n+1)}\right) \quad(i=1, \ldots, l) .
\end{gathered}
$$

Then $\left(C_{1}^{(n)}, C_{2}^{(n)}, \Phi_{C^{(n)}}, F_{*}\left(C_{1}^{(n)}\right)\right)$ becomes a parabolic $\Lambda_{\mathcal{D}_{k}}^{1}$-triple and $F_{i+1}\left(C_{1}^{(n)}\right)=C_{1}^{(n)} \cap\left(F_{i+1}\left(E_{1}^{(n+1)}\right) \otimes k\right)$ for $i=1, \ldots, l$. A quotient parabolic structure $F_{*}\left(B_{1}^{(n+1)} / C_{1}^{(n)}\right)$ is induced on $B_{1}^{(n+1)} / C_{1}^{(n)}$ and a homomorphism $\Phi_{B^{(n+1)} / C^{(n)}}: \Lambda_{\mathcal{D}_{k}}^{1} \otimes B_{1}^{(n+1)} / C_{1}^{(n)} \rightarrow B_{2}^{(n+1)} / C_{2}^{(n)}$ is induced by $\Phi^{(n+1)}$. Then

$$
\left(B_{1}^{(n+1)} / C_{1}^{(n)}, B_{2}^{(n+1)} / C_{2}^{(n)}, \Phi_{B^{(n+1)} / C^{(n)}}, F_{*}\left(B_{1}^{(n+1)} / C_{1}^{(n)}\right)\right)
$$

becomes a parabolic $\Lambda_{\mathcal{D}_{k}}^{1}$-triple. If $\left(C_{1}^{(n)}, C_{2}^{(n)}\right) \neq(0,0)$, then we have

$$
\begin{gathered}
\\
\mu\left(\left(C_{1}^{(n)}, C_{2}^{(n)}, \Phi_{C^{(n)}}, F_{*}\left(C_{1}^{(n)}\right)\right)\right) \leq \mu_{\max }\left(\left(G_{1}^{(n)}, G_{2}^{(n)}, \Phi_{G^{(n)},}, F_{*}\left(G_{*}^{(n)}\right)\right)\right) \\
<\mu_{\max }\left(\left(E_{1}^{(n)}, E_{2}^{(n)}, \Phi^{(n)}, F_{*}\left(E_{1}^{(n)}\right)\right) \otimes k\right)=\mu\left(\left(B_{1}^{(n)}, B_{2}^{(n)}, \Phi_{B^{(n)}}, F_{*}\left(B_{1}^{(n)}\right)\right)\right),
\end{gathered}
$$

where $\mu_{\max }$ means the value of $\mu$ at the maximal destabilizer. Thus, in any case, we have the inequality

$$
\begin{aligned}
& \mu\left(\left(B_{1}^{(n+1)}, B_{2}^{(n+1)}, \Phi_{B^{(n+1)}}, F_{*}\left(B_{1}^{(n+1)}\right)\right)\right) \\
\leq & \mu\left(\left(B_{1}^{(n+1)} / C_{1}^{(n)}, B_{2}^{(n+1)} / C_{2}^{(n)}, \Phi_{B^{(n+1)} / C^{(n)}}, F_{*}\left(B_{1}^{(n+1)} / C_{1}^{(n)}\right)\right)\right) \\
\leq & \mu\left(\left(B_{1}^{(n)}, B_{2}^{(n)}, \Phi_{B^{(n)}}, F_{*}\left(B_{1}^{(n)}\right)\right)\right)
\end{aligned}
$$

with equality if and only if $\left(C_{1}^{(n)}, C_{2}^{(n)}\right)=(0,0)$.

The descending sequence

$$
\left\{\mu\left(\left(B_{1}^{(n)}, B_{2}^{(n)}, \Phi_{B^{(n)}}, F_{*}\left(B_{1}^{(n)}\right)\right)\right)\right\}_{n \in \mathbf{N}}
$$

must become stationary since it is bounded below. We may assume without loss of generality that

$$
\mu\left(\left(B_{1}^{(n)}, B_{2}^{(n)}, \Phi_{B^{(n)}}, F_{*}\left(B_{1}^{(n)}\right)\right)\right)
$$


is constant for all $n$. In this case we must have $\left(C_{1}^{(n)}, C_{2}^{(n)}\right)=(0,0)$ and

$$
\left(B_{1}^{(n+1)}, B_{2}^{(n+1)}, \Phi_{B^{(n+1)}}, F_{*}\left(B_{1}^{(n+1)}\right)\right)
$$

becomes a parabolic $\Lambda_{\mathcal{D}_{k}}^{1}$-subtriple of

$$
\left(B_{1}^{(n)}, B_{2}^{(n)}, \Phi_{B^{(n)}}, F_{*}\left(B_{1}^{(n)}\right)\right)
$$

for all $n$. Since the descending sequence $\left\{\operatorname{rank} B_{1}^{(n)}+\operatorname{rank} B_{2}^{(n)}\right\}_{n \in \mathbf{N}}$ must be stationary, we may assume without loss of generality that $\operatorname{rank} B_{1}^{(n)}+\operatorname{rank} B_{2}^{(n)}$ is constant for all $n$. Then we must have

$$
\left(B_{1}^{(n)}, B_{2}^{(n)}, \Phi_{B^{(n)}}, F_{*}\left(B_{1}^{(n)}\right)\right)=\left(B_{1}^{(n+1)}, B_{2}^{(n+1)}, \Phi_{B^{(n+1)}}, F_{*}\left(B_{1}^{(n+1)}\right)\right)
$$

for all $n$. Thus the sequences (107) split and

$$
\begin{aligned}
\left(E_{1}^{(n)}, E_{2}^{(n)}, \Phi^{(n)}\right. & \left., F_{*}\left(E_{1}^{(n)}\right)\right) \otimes k \\
& \cong\left(B_{1}^{(n)}, B_{2}^{(n)}, \Phi_{B^{(n)}}, F_{*}\left(B_{1}^{(n)}\right)\right) \oplus\left(G_{1}^{(n)}, G_{2}^{(n)}, \Phi_{G^{(n)}}, F_{*}\left(G_{1}^{(n)}\right)\right) .
\end{aligned}
$$

Then all the maps $G_{j}^{(n)} \rightarrow G_{j}^{(n+1)}$ are isomorphisms. Since $B_{j}^{(n+1)} \rightarrow B_{j}^{(n)}$ are all isomorphic, every image of $E_{j}^{(n)} \otimes k \rightarrow E_{j}^{(0)} \otimes k$ is $B_{j}^{(0)}$ for $j=1,2$. So we have an isomorphism $\left(E_{j}^{(0)} / E_{j}^{(n)}\right) \otimes k \cong G_{j}^{(0)}$ for any $n$. On the other hand, every image of $m^{n} / m^{n+1} \otimes E_{j}^{(0)} \rightarrow E_{j}^{(n)} \otimes k$ is $G_{j}^{(n-1)}$. So we have an isomorphism $\left(E_{j}^{(n)} / m^{n} E_{j}^{(0)}\right) \otimes k \cong B_{j}^{(n-1)}$. Consider the exact sequence

$$
0 \longrightarrow E_{j}^{(n)} / m^{n} E_{j}^{(0)} \stackrel{u}{\longrightarrow} E_{j}^{(0)} / m^{n} E_{j}^{(0)} \longrightarrow E_{j}^{(0)} / E_{j}^{(n)} \longrightarrow 0 .
$$

Then $u \otimes k:\left(E_{j}^{(n)} / m^{n} E_{j}^{(0)}\right) \otimes k \cong B_{j}^{(n-1)} \rightarrow E_{j}^{(0)} \otimes k$ is injective. Thus $u$ is injective and $E_{j}^{(0)} / E_{j}^{(n)}$ is flat over $R / m^{n} R$. Then quotients $E_{j}^{(0)} \otimes R / m^{n} \rightarrow$ $E_{j}^{(0)} / E_{j}^{(n)}$ define a system of morphisms Spec $R / m^{n} \rightarrow Q_{j}^{\prime}:=\operatorname{Quot}_{E_{j}^{(0)} / \mathcal{X}_{R} / R}^{\chi\left(G^{(0)}(n)\right)}$ which induces a morphism $f_{j}: \operatorname{Spec} \hat{R} \rightarrow Q_{j}^{\prime}$, where $\hat{R}$ is the completion of $R$. If $\tilde{G}_{j}$ is the quotient sheaf of $E_{j}^{(0)} \otimes \hat{R}$ corresponding to $f_{j}$, then we have $\tilde{G}_{j} \otimes$ $R / m^{n} R \cong E_{j}^{(0)} / E_{j}^{(n)}$. Similarly we can lift the parabolic structure $F_{*}\left(G_{1}^{(0)}\right)$ to a flat family $F_{*}\left(\tilde{G}_{1}\right)$ of parabolic structure on $\tilde{G}_{1}$ over $\hat{R}$. We can also lift $\Phi_{G^{(0)}}$ to $\Phi_{\tilde{G}}: \Lambda_{\mathcal{D} / S}^{1} \otimes \tilde{G}_{1} \rightarrow \tilde{G}_{2}$ and $\left(\tilde{G}_{1}, \tilde{G}_{2}, \Phi_{\tilde{G}}, F_{*}\left(\tilde{G}_{1}\right)\right)$ becomes a flat family of parabolic $\Lambda_{\mathcal{D}_{R}}^{1}$-triples which is a quotient of $\left(E_{1}^{(0)}, E_{2}^{(0)}, \Phi^{(0)}, F_{*}\left(E_{*}^{(0)}\right)\right) \otimes \hat{R}$. If $\hat{K}$ is the quotient field of $\hat{R}$, then $\left(\tilde{G}_{1}, \tilde{G}_{2}, \Phi_{\tilde{G}}, F_{*}\left(\tilde{G}_{1}\right)\right) \otimes \hat{K}$ becomes a destabilizing quotient parabolic $\Lambda_{\mathcal{D}_{\hat{K}}}^{1}$-triple of $\left(E_{1}, E_{2}, \Phi, F_{*}\left(E_{1}\right)\right) \otimes \hat{K}$, which contradicts the semistability of $\left(E_{1}, E_{2}, \Phi, F_{*}\left(E_{1}\right)\right)$.

As a corollary of Proposition 5.5, we obtain the following proposition: 
Proposition 5.6. Assume that $\alpha_{1}^{\prime}, \ldots, \alpha_{l}^{\prime}$ are sufficiently general so that all the semistable parabolic $\Lambda_{\mathcal{D} / S^{-}}^{1}$ triples are stable. Then the moduli scheme $\overline{M_{\mathcal{X} / S}^{\mathcal{D}, \boldsymbol{\alpha}^{\prime}, \boldsymbol{\beta}, \gamma}}\left(r, d,\left\{d_{i}\right\}\right)$ is projective over $S$.

There is another corollary of Proposition 5.5 which is used in the proof of the surjectivity of the Riemann-Hilbert morphism in Lemma 7.1. For a parabolic connection $\left(E, \nabla, \varphi,\left\{l_{i}\right\}\right)$, let $(0,0)=\left(F_{0}, \nabla_{0}\right) \subset\left(F_{1}, \nabla_{1}\right) \subset \cdots \subset$ $\left(F_{l}, \nabla_{l}\right)=(E, \nabla)$ be a Jordan-Hölder filtration of $(E, \nabla)$, that is, each $\left(F_{i} / F_{i+1}\right.$, $\left.\overline{\nabla_{i}}\right)$ is irreducible, where $\overline{\nabla_{i}}: F_{i} / F_{i+1} \rightarrow F_{i} / F_{i+1} \otimes \Omega_{X}^{1}(D(\mathbf{t}))$ is the connection induced by $\nabla_{i}$. Then we put

$$
\operatorname{gr}(E, \nabla):=\bigoplus_{i=1}^{l}\left(F_{i} / F_{i+1}, \overline{\nabla_{i}}\right) .
$$

Corollary 5.1. Let $R$ be a discrete valuation ring with quotient field $K$ and residue field $k$. Let $\left(E, \nabla, \varphi,\left\{l_{i}\right\}\right)$ be a flat family of connections with parabolic structures on $X \times \operatorname{Spec} R$ over $R$ such that the generic fiber $(E, \nabla$, $\left.\varphi,\left\{l_{i}\right\}\right) \otimes_{R} K$ is $\boldsymbol{\alpha}$-semistable. Then there exists a flat family $\left(\tilde{E}, \tilde{\nabla}, \tilde{\varphi},\left\{\tilde{l}_{i}\right\}\right)$ of $\boldsymbol{\alpha}$-semistable parabolic connections such that $\left(\tilde{E}, \tilde{\nabla}, \tilde{\varphi},\left\{\tilde{l}_{i}\right\}\right) \otimes K \cong\left(E, \nabla, \varphi,\left\{l_{i}\right\}\right)$ $\otimes K$ and $\operatorname{gr}((\tilde{E}, \tilde{\nabla}) \otimes k) \cong \operatorname{gr}((E, \nabla) \otimes k)$.

\section{§5.5. Proof of Theorem 2.1 (1)}

Now we prove the assertion (1) of Theorem 2.1.

We take $S$ for $T_{n} \times \Lambda_{n}$ and $\mathcal{X}$ for $\mathbf{P}^{1} \times T_{n} \times \Lambda_{n}$.

Let $\mathcal{D}_{i} \subset \mathbf{P}^{1} \times T_{n} \times \Lambda_{n}$ be the effective divisor determined by the section $T_{n} \times \Lambda_{n} \hookrightarrow \mathbf{P}^{1} \times T_{n} \times \Lambda_{n} ; \quad\left(\left(t_{j}\right)_{1 \leq j \leq n},\left(\lambda_{k}\right)_{1 \leq k \leq n}\right) \mapsto\left(t_{i},\left(t_{j}\right)_{1 \leq j \leq n},\left(\lambda_{k}\right)_{1 \leq k \leq n}\right)$ for $i=1, \ldots, n$ and put $\mathcal{D}:=\sum_{i=1}^{n} \mathcal{D}_{i}$. Then $\mathcal{D}$ becomes an effective Cartier divisor on $\mathbf{P}^{1} \times T_{n} \times \Lambda_{n}$ which is flat over $T_{n} \times \Lambda_{n}$.

We fix a line bundle $L$ on $\mathbf{P}^{1} \times T_{n} \times \Lambda_{n}$ with a relative connection

$$
\nabla_{L}: L \rightarrow L \otimes \Omega_{\mathbf{P}^{1} \times T_{n} \times \Lambda_{n} / T_{n} \times \Lambda_{n}}^{1}
$$

over $T_{n} \times \Lambda_{n}$. Let $\boldsymbol{\alpha}^{\prime}=\left(\alpha_{1}^{\prime}, \ldots, \alpha_{2 n}^{\prime}\right), \boldsymbol{\beta}=\left(\beta_{1}, \beta_{2}\right)$, and $\gamma \gg 0$ be as in Theorem 2.1 .

We define a moduli functor $\overline{\mathcal{M}_{n}^{\boldsymbol{\alpha}^{\prime} \boldsymbol{\beta}}}(L)$ of the category of locally noetherian schemes over $T_{n} \times \Lambda_{n}$ to the category of sets by

$$
\overline{\mathcal{M}_{n}^{\boldsymbol{\alpha}^{\prime} \boldsymbol{\beta}}}(L)(S):=\left\{\left(E_{1}, E_{2}, \phi, \nabla, \varphi,\left\{l_{i}\right\}_{i=1}^{n}\right)\right\} / \sim,
$$

where $S$ is a locally noetherian scheme over $T_{n} \times \Lambda_{n}$ corresponding to $(\mathbf{t}, \boldsymbol{\lambda})=$ $\left(t_{1}, \ldots, t_{n}, \lambda_{1}, \ldots, \lambda_{n}\right) \in T_{n}(S) \times \Lambda_{n}(S)$ and 
(1) $E_{1}, E_{2}$ are rank 2 vector bundles on $\mathbf{P}^{1} \times S$,

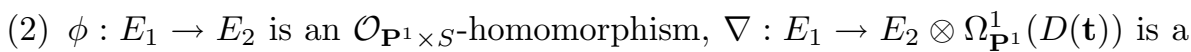
morphism such that $\nabla(f a)=\phi(a) \otimes d f+f \nabla(a)$ for $f \in \mathcal{O}_{\mathbf{P}^{1} \times S}, a \in E_{1}$,

(3) $\left.l_{i} \subset E_{1}\right|_{t_{i}}$ are rank 1 subbundles such that $\left.\left(\operatorname{res}_{t_{i}}(\nabla)-\left.\lambda_{i} \phi\right|_{t_{i}}\right)\right|_{l_{i}}=0$ for $i=1, \ldots, n$,

(4) $\varphi: \bigwedge^{2} E_{2} \stackrel{\sim}{\longrightarrow} L \otimes \mathcal{L}_{\varphi}$ is an isomorphism such that $(\varphi \otimes 1)\left(\nabla\left(s_{1}\right) \wedge \phi\left(s_{2}\right)+\right.$ $\left.\phi\left(s_{1}\right) \wedge \nabla\left(s_{2}\right)\right)=\left(\nabla_{L} \otimes \operatorname{id}_{\mathcal{L}_{\varphi}}\right)\left(\varphi\left(\phi\left(s_{1}\right) \wedge \phi\left(s_{2}\right)\right)\right)$ for $s_{1}, s_{2} \in E_{1}$ where $\mathcal{L}_{\varphi}$ is a line bundle on $S$,

(5) for any geometric point $s$ of $S$, the fiber $\left(\left(E_{1}\right)_{s},\left(E_{2}\right)_{s}, \phi_{s}, \nabla_{s}, \varphi_{s}\right.$, $\left.\left\{\left.l_{i}\right|_{t_{i} \otimes k(s)}\right\}_{i=1}^{n}\right)$ is $\left(\boldsymbol{\alpha}^{\prime}, \boldsymbol{\beta}\right)$-stable and $\operatorname{deg}\left(E_{1}\right)_{s}=\operatorname{deg} L_{s}$.

Here $\left(E_{1}, E_{2}, \phi, \nabla, \varphi,\left\{l_{i}\right\}\right) \sim\left(E_{1}^{\prime}, E_{2}^{\prime}, \phi^{\prime}, \nabla^{\prime}, \varphi^{\prime},\left\{l_{i}^{\prime}\right\}\right)$ if there exist a line bundle $\mathcal{L}$ on $S$ and isomorphisms $\sigma_{j}: E_{j} \stackrel{\sim}{\rightarrow} E_{j}^{\prime} \otimes \mathcal{L}$ for $j=1,2$ such that $\left.\sigma_{1}\right|_{t_{i} \times S}\left(l_{i}\right)=l_{i}^{\prime}$ for any $i$, the diagrams

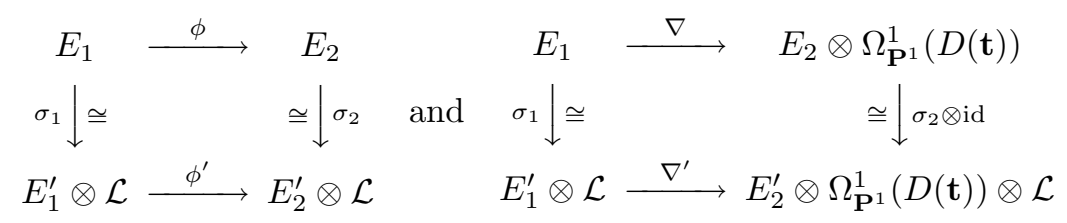

commute and there is an isomorphism $\sigma: \mathcal{L}_{\varphi} \stackrel{\sim}{\rightarrow} \mathcal{L}_{\varphi^{\prime}} \otimes \mathcal{L}^{\otimes 2}$ such that the diagram

$$
\begin{array}{ccc}
\bigwedge^{2} E_{2} & \stackrel{\varphi}{\sim} & L \otimes \mathcal{L}_{\varphi} \\
\wedge \sigma_{2} \downarrow \cong & \cong \downarrow \operatorname{id} \otimes \sigma \\
\bigwedge^{2} E_{2}^{\prime} \otimes \mathcal{L} & \stackrel{\varphi^{\prime} \otimes \operatorname{id}_{\mathcal{L}}}{\sim} L \otimes \mathcal{L}_{\varphi^{\prime}} \otimes \mathcal{L}^{\otimes 2}
\end{array}
$$

commutes.

We can define another weight $\boldsymbol{\alpha}=\left(\alpha_{1}, \ldots, \alpha_{2 n}\right)$ with $0 \leq \alpha_{1}<\cdots<$ $\alpha_{2 n}<\frac{\beta_{1}}{\beta_{1}+\beta_{2}}<1$ by

$$
\boldsymbol{\alpha}=\boldsymbol{\alpha}^{\prime} \frac{\beta_{1}}{\beta_{1}+\beta_{2}} .
$$

Theorem 2.1, (1) follows from the following theorem:

Theorem 5.2. There exists a coarse moduli scheme $\overline{M_{n}^{\boldsymbol{\alpha}^{\prime} \boldsymbol{\beta}}}(L)$ of $\overline{\mathcal{M}_{n}^{\boldsymbol{\alpha}^{\prime} \boldsymbol{\beta}}}(L)$, which is projective over $T_{n} \times \Lambda_{n}$ if $\boldsymbol{\alpha}^{\prime}$ is generic. If we put 


$$
\begin{aligned}
& M_{n}^{\boldsymbol{\alpha}}(L) \\
& \quad:=\left\{\left(E_{1}, E_{2}, \phi, \nabla, \varphi,\left\{l_{i}\right\}\right) \in \overline{M_{n}^{\boldsymbol{\alpha}^{\prime} \boldsymbol{\beta}}}(L) \mid \phi: E_{1} \rightarrow E_{2} \text { is an isomorphism }\right\},
\end{aligned}
$$

then $M_{n}^{\boldsymbol{\alpha}}(L)$ is a Zariski open subset of $\overline{M_{n}^{\boldsymbol{\alpha}^{\prime} \boldsymbol{\beta}}}(L)$, which is a fine moduli scheme of $\boldsymbol{\alpha}$-stable parabolic connections.

Proof. We put $r=2, d=\operatorname{deg} L_{s}$ for $s \in T_{n} \times \Lambda_{n}, l=2 n$ and $d_{i}=i$ for $i=1, \ldots, 2 n$ and consider the moduli scheme $\frac{n}{M_{\mathbf{P}^{1} \times T_{n} \times \Lambda_{n} / T_{n} \times \Lambda_{n}}^{\mathcal{D}, \boldsymbol{\alpha}^{\prime}, \boldsymbol{\beta}, \gamma}}\left(2, d,\left\{d_{i}\right\}\right)$. For each $\left(E_{1}, E_{2}, \phi, \nabla, \varphi,\left\{l_{i}\right\}\right) \in \overline{\mathcal{M}_{n}^{\boldsymbol{\alpha}^{\prime} \boldsymbol{\beta}}}(L)(S)$, let $\Phi: \Lambda_{\mathcal{D}_{S}}^{1} \otimes E_{1} \rightarrow E_{2}$ be

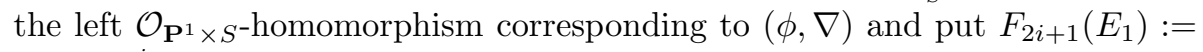
$E_{1}\left(-\sum_{j=1}^{i} t_{j}\right)$ for $i=0, \ldots, l$ and $F_{2 i}\left(E_{1}\right):=\operatorname{ker}\left(F_{2 i-1}\left(E_{1}\right) \rightarrow\left(\left.E_{1}\right|_{t_{i}} / l_{i}\right)\right)$ for $i=1, \ldots, l$, where $\left(t_{1}, \ldots, t_{n}, \lambda_{1}, \ldots, \lambda_{n}\right) \in T_{n}(S) \times \Lambda_{n}(S)$ corresponds to the structure morphism $S \rightarrow T_{n} \times \Lambda_{n}$. Then the correspondence $\left(E_{1}, E_{2}, \phi, \nabla\right.$, $\left.\varphi,\left\{l_{i}\right\}\right) \mapsto\left(E_{1}, E_{2}, \Phi, F_{*}\left(E_{1}\right)\right)$ determines a morphism of functors

$$
\iota: \overline{\mathcal{M}_{n}^{\boldsymbol{\alpha}^{\prime} \boldsymbol{\beta}}}(L) \rightarrow \overline{\mathcal{M}_{\mathbf{P}^{1} \times T_{n} \times \Lambda_{n} / T_{n} \times \Lambda_{n}}^{\mathcal{D}, \boldsymbol{\alpha}^{\prime}, \boldsymbol{\beta}, \gamma}}\left(2, d,\left\{d_{i}\right\}\right) .
$$

We can easily see that $\iota$ is represented by a closed immersion. Recall that $R^{s} \rightarrow \overline{M_{\mathbf{P}^{1} \times T_{n} \times \Lambda_{n} / T_{n} \times \Lambda_{n}}^{\mathcal{D}, \boldsymbol{\alpha}^{\prime}, \boldsymbol{\beta}, \gamma}}\left(2, d,\left\{d_{i}\right\}\right)$ is a principal $G$-bundle. Then there exists a closed subscheme $Z \subset R^{s}$ such that

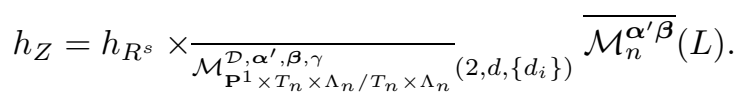

$Z$ descends to a closed subscheme of $\overline{M_{\mathbf{P}^{1} \times T_{n} \times \Lambda_{n} / T_{n} \times \Lambda_{n}}^{\mathcal{D}, \boldsymbol{\alpha}^{\prime}, \boldsymbol{\beta}, \gamma}}\left(2, d,\left\{d_{i}\right\}\right)$ which is just the coarse moduli scheme of $\overline{\mathcal{M}_{n}^{\boldsymbol{\alpha}^{\prime} \boldsymbol{\beta}}}(L)$.

If we take $\gamma$ sufficiently large, we can check that a parabolic connection $\left(E, \nabla_{E}, \varphi,\left\{l_{i}\right\}\right)$ is $\boldsymbol{\alpha}$-stable if and only if the associated parabolic $\phi$-connection $\left(E, E, \operatorname{id}_{E}, \nabla_{E}, \varphi,\left\{l_{i}\right\}\right)$ is $\left(\boldsymbol{\alpha}^{\prime}, \boldsymbol{\beta}\right)$-stable. Thus the open subscheme

$$
\begin{aligned}
& M_{n}^{\boldsymbol{\alpha}}(L) \\
& \quad:=\left\{\left(E_{1}, E_{2},(\phi, \nabla), F_{*}\left(E_{1}\right)\right) \in \overline{M_{n}^{\boldsymbol{\alpha}^{\prime} \boldsymbol{\beta}}}(L) \mid \phi: E_{1} \rightarrow E_{2} \text { is an isomorphism }\right\}
\end{aligned}
$$

of $\overline{M_{n}^{\boldsymbol{\alpha}^{\prime} \boldsymbol{\beta}}}(L)$ is just the moduli space of $\boldsymbol{\alpha}$-stable parabolic connections with the determinant $L$.

If $\operatorname{deg} L$ is odd, we can see by the same argument as [[M], Theorem 6.11] or [[HL], Theorem 4.6.5] that $M_{n}^{\boldsymbol{\alpha}}(L)$ is in fact a fine moduli scheme. If $\operatorname{deg} L$ is even, then we can obtain, by an elementary transform, an isomorphism

$$
\sigma: \mathcal{M}_{n}(L) \rightarrow \mathcal{M}_{n}\left(L^{\prime}\right)
$$


of moduli stacks of parabolic connections without stability condition, where $\operatorname{deg} L^{\prime}$ is odd. Then we can see by the same argument that $\sigma\left(M_{n}^{\boldsymbol{\alpha}}(L)\right)$ becomes a fine moduli scheme, and so $M_{n}^{\boldsymbol{\alpha}}(L)$ is also fine.

\section{§6. Tangent Spaces of the Moduli Spaces and Canonical Symplectic Structure}

In this section, we will work over the finite étale covering $T_{n}^{\prime} \longrightarrow T_{n}$ defined in (94). Fix $(\mathbf{t}, \boldsymbol{\lambda}) \in T_{n}^{\prime} \times \Lambda_{n}$ and set $a_{i}=2 \cos 2 \pi \lambda_{i}$ and $\mathbf{a}=\left(a_{1}, \ldots, a_{n}\right)$. Moreover fix a determinant line bundle $L=\left(L, \nabla_{L}\right)$ on $\mathbf{P}^{1}$ such that $\operatorname{res}_{t_{i}}\left(\nabla_{L}\right) \in \mathbf{Z}$. We have defined two moduli spaces $M_{n}^{\boldsymbol{\alpha}}(\mathbf{t}, \boldsymbol{\lambda}, L), \mathcal{R}\left(\mathcal{P}_{n, \mathbf{t}}\right)_{\mathbf{a}}$ where $M_{n}^{\boldsymbol{\alpha}}(\mathbf{t}, \boldsymbol{\lambda}, L)$ is the moduli space of stable $(\mathbf{t}, \boldsymbol{\lambda})$-parabolic connections with the determinant $L$ and $\mathcal{R}\left(\mathcal{P}_{n, \mathbf{t}}\right)_{\mathbf{a}}$ is the moduli of Jordan equivalence classes of the $S L_{2}(\mathbf{C})$ representations of $\pi_{1}\left(\mathbf{P}^{1} \backslash D(\mathbf{t}), *\right)$ with fixed local exponents $\mathbf{a}=\left(a_{1}, \ldots, a_{n}\right)$. As we show in Theorem 2.1, for a suitable (or generic) weight $\boldsymbol{\alpha}$, the moduli space $M_{n}^{\boldsymbol{\alpha}}(\mathbf{t}, \boldsymbol{\lambda}, L)$ is a non-singular complex scheme. In this section, we will describe the tangent space to $M_{n}^{\boldsymbol{\alpha}}(\mathbf{t}, \boldsymbol{\lambda}, L)$ and a non-degenerate holomorphic 2-form on the moduli space $M_{n}^{\boldsymbol{\alpha}}(\mathbf{t}, \boldsymbol{\lambda}, L)$.

Although the moduli space $\mathcal{R}\left(\mathcal{P}_{n, \mathbf{t}}\right)_{\mathbf{a}}$ may be singular, we can define a Zariski dense open set $\mathcal{R}\left(\mathcal{P}_{n, \mathbf{t}}\right)_{\mathbf{a}}^{\sharp}$ of $\mathcal{R}\left(\mathcal{P}_{n, \mathbf{t}}\right)_{\mathbf{a}}$ such that $\mathcal{R}\left(\mathcal{P}_{n, \mathbf{t}}\right)_{\mathbf{a}}^{\sharp}$ is a nonsingular variety. (Note that for generic $\left.\mathbf{a} \in \mathcal{A}_{n}, \mathcal{R}\left(\mathcal{P}_{n, \mathbf{t}}\right)_{\mathbf{a}}^{\sharp}=\mathcal{R}\left(\mathcal{P}_{n, \mathbf{t}}\right)_{\mathbf{a}}\right)$. Moreover on $\mathcal{R}\left(\mathcal{P}_{n, \mathbf{t}}\right)_{\mathbf{a}}^{\sharp}$ we can also define a canonical symplectic structure $\Omega_{1}$. In $\S 7$ we define the Riemann-Hilbert correspondence $\mathbf{R H}_{\mathbf{t}, \boldsymbol{\lambda}}: M_{n}^{\boldsymbol{\alpha}}(\mathbf{t}, \boldsymbol{\lambda}, L) \longrightarrow$ $\mathcal{R}\left(\mathcal{P}_{n, \mathbf{t}}\right)_{\mathbf{a}}$. We show that $\mathbf{R H}_{\mathbf{t}, \boldsymbol{\lambda}}$ is bimeromorphic proper surjective morphism and gives an analytic isomorphism between $M_{n}^{\boldsymbol{\alpha}}(\mathbf{t}, \boldsymbol{\lambda}, L)^{\sharp}:=\mathbf{R H}_{\mathbf{t}, \boldsymbol{\lambda}}^{-1}\left(\mathcal{R}\left(\mathcal{P}_{n, \mathbf{t}}\right)_{\mathbf{a}}^{\sharp}\right)$ and $\mathcal{R}\left(\mathcal{P}_{n, \mathbf{t}}\right)_{\mathbf{a}}^{\sharp}$. (Again, for a generic $\left.\boldsymbol{\lambda}, M_{n}^{\boldsymbol{\alpha}}(\mathbf{t}, \boldsymbol{\lambda}, L)^{\sharp}=M_{n}^{\boldsymbol{\alpha}}(\mathbf{t}, \boldsymbol{\lambda}, L)\right)$. Note that $\mathbf{R H}_{\mathbf{t}, \boldsymbol{\lambda}}$ is not an algebraic morphism, and hence the algebraic structures of $M_{n}^{\boldsymbol{\alpha}}(\mathbf{t}, \boldsymbol{\lambda}, L)^{\sharp}$ and $\mathcal{R}\left(\mathcal{P}_{n, \mathbf{t}}\right)_{\mathbf{a}}^{\sharp}$ are completely different $)$. The canonical symplectic

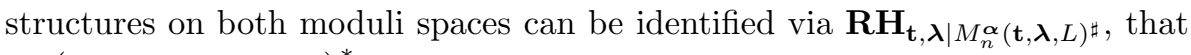
is, $\left(\mathbf{R H}_{\mathbf{t}, \boldsymbol{\lambda} \mid M_{n}^{\alpha}(\mathbf{t}, \boldsymbol{\lambda}, L)^{\sharp}}\right)^{*}\left(\Omega_{1}\right)=\Omega$.

\section{$\S 6.1$. Tangent space to $M_{n}^{\boldsymbol{\alpha}}(\mathbf{t}, \boldsymbol{\lambda}, L)$}

Consider the base extension of the family of moduli spaces in (48) by the étale covering $T_{n}^{\prime} \longrightarrow T_{n}$ :

$$
\pi_{n}: M_{n}^{\alpha}(L) \longrightarrow T_{n}^{\prime} \times \Lambda_{n},
$$

such that for every $(\mathbf{t}, \boldsymbol{\lambda}) \in T_{n}^{\prime} \times \Lambda_{n}$, we have $\pi_{n}^{-1}((\mathbf{t}, \lambda)) \simeq M_{n}^{\boldsymbol{\alpha}}(\mathbf{t}, \boldsymbol{\lambda}, L)$. For simplicity, we will omit $L$ from now on, so we write as $M_{n}^{\boldsymbol{\alpha}}=M_{n}^{\boldsymbol{\alpha}}(L)$, 
$M_{n}^{\boldsymbol{\alpha}}(\mathbf{t}, \boldsymbol{\lambda})=M_{n}^{\boldsymbol{\alpha}}(\mathbf{t}, \boldsymbol{\lambda}, L)$. We assume that $\boldsymbol{\alpha}$ is generic so that $\pi_{n}$ is a smooth morphism (cf. Theorem 2.1).

Let us consider natural projection maps

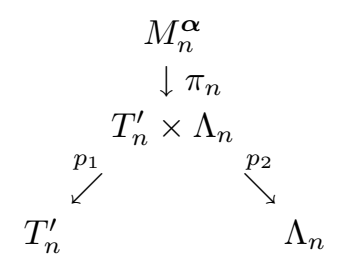

and set $\varphi_{i}=p_{i} \circ \pi_{n}$. Since $\varphi_{1}: M_{n}^{\alpha} \longrightarrow T_{n}^{\prime}$ is smooth, we have the following exact sequence of tangent sheaves on $M_{n}^{\alpha}$

$$
0 \longrightarrow \Theta_{M_{n}^{\alpha} / T_{n}^{\prime} \times \Lambda_{n}} \longrightarrow \Theta_{M_{n}^{\alpha} / T_{n}^{\prime}} \longrightarrow \pi_{n}^{*}\left(\Theta_{T_{n}^{\prime} \times \Lambda_{n} / T_{n}^{\prime}}\right) \longrightarrow 0 .
$$

We will describe this exact sequence in terms of the infinitesimal deformation of the stable parabolic connections. Let us consider the natural projection map $q_{2}: \mathbf{P}^{1} \times T_{n}^{\prime} \longrightarrow T_{n}^{\prime}$ and defines a divisor $\mathcal{D} \subset \mathbf{P}^{1} \times T_{n}^{\prime}$ such that $q_{2}^{-1}(\mathbf{t}) \cap \mathcal{D}=$ $D(\mathbf{t})=t_{1}+\cdots+t_{n} \subset \mathbf{P}^{1}$.

Let $\left(\tilde{E}, \tilde{\nabla}, \tilde{\varphi},\left\{\tilde{l}_{i}\right\}\right)$ be a universal family on $\mathbf{P}^{1} \times M_{n}^{\alpha}$. Consider the following commutative diagram:

$$
\begin{aligned}
& \begin{array}{lc} 
& \mathbf{P}^{1} \times M_{n}^{\boldsymbol{\alpha}} \\
\tilde{\varphi}_{1} & \downarrow \tilde{\pi}_{n}
\end{array} \\
& \mathbf{P}^{1} \times T_{n}^{\prime} \times \Lambda_{n} \\
& \mathbf{P}^{1} \times T_{n}^{\prime} \\
& q_{2} \downarrow \\
& T_{n}^{\prime}
\end{aligned}
$$

For a coherent sheaf $\mathcal{G}$ on $\mathbf{P}^{1} \times M_{n}^{\alpha}$ and a closed point $\mathbf{x} \in M_{n}^{\alpha}$, we set $\mathcal{G}_{\mathbf{x}}:=\mathcal{G}_{\mid \mathbf{P}^{1} \times \mathbf{x}}$.

We define coherent sheaves on $\mathbf{P}^{1} \times M_{n}^{\alpha}$ as follows.

$$
\mathcal{F}^{0}:=\left\{s \in \mathcal{E} n d(\tilde{E}) \mid \operatorname{Tr}(s)=0, ;\left(\left.s\right|_{t_{i} \times M_{n}^{\alpha}}\right)\left(\tilde{l}_{i}\right) \subset \tilde{l}_{i}\right\}
$$

$$
\mathcal{F}^{1}:=\left\{s \in \mathcal{E} n d(\tilde{E}) \otimes \tilde{\varphi}_{1}^{*}\left(\Omega_{\mathbf{P}^{1} \times T_{n}^{\prime} / T_{n}^{\prime}}^{1}(\mathcal{D})\right) \mid \operatorname{Tr}(s)=0, \quad\left(\left.s\right|_{t_{i} \times M_{n}^{\alpha}}\right)\left(\tilde{l}_{i}\right)=0\right\}
$$

$$
\mathcal{F}^{1,+}:=\left\{s \in \mathcal{E} n d(\tilde{E}) \otimes \tilde{\varphi}_{1}^{*}\left(\Omega_{\mathbf{P}^{1} \times T_{n}^{\prime} / T_{n}^{\prime}}^{1}(\mathcal{D})\right) \mid \operatorname{Tr}(s)=0, \operatorname{res}_{\left(t_{i} \times M_{n}^{\alpha}\right)}(s)\left(\tilde{l}_{i}\right) \subset \tilde{l}_{i}\right\}
$$


For a local section $s$ of $\mathcal{F}^{0}$, define $\nabla_{1}(s):=\tilde{\nabla} s-s \tilde{\nabla}$. Then it is easy to see that $\nabla_{1}(s)$ is a local section of $\mathcal{F}^{1}$. Since we have a natural inclusion of sheaves $\iota: \mathcal{F}^{1} \hookrightarrow \mathcal{F}^{1,+}$, we can define two complexes of sheaves on $\mathbf{P}^{1} \times M_{n}^{\alpha}$ :

$$
\begin{aligned}
\mathcal{F}^{\bullet} & :=\left[\nabla_{1}: \mathcal{F}^{0} \longrightarrow \mathcal{F}^{1}\right], \\
\mathcal{F}^{\bullet},+ & :=\left[\nabla_{1}^{+}: \mathcal{F}^{0} \longrightarrow \mathcal{F}^{1,+}\right] .
\end{aligned}
$$

Let $\mathbf{x} \in M_{n}^{\alpha}$ be a closed point and set $\pi(\mathbf{x})=(\mathbf{t}, \boldsymbol{\lambda})$. Setting $\mathcal{T}_{1}=$ $\mathcal{F}^{1,+} / \mathcal{F}^{1}$, we have the following exact sequences of the complexes on $\mathbf{P}^{1} \times M_{n}^{\boldsymbol{\alpha}}$ and $\mathbf{P}^{1} \times\{\mathbf{x}\}$.
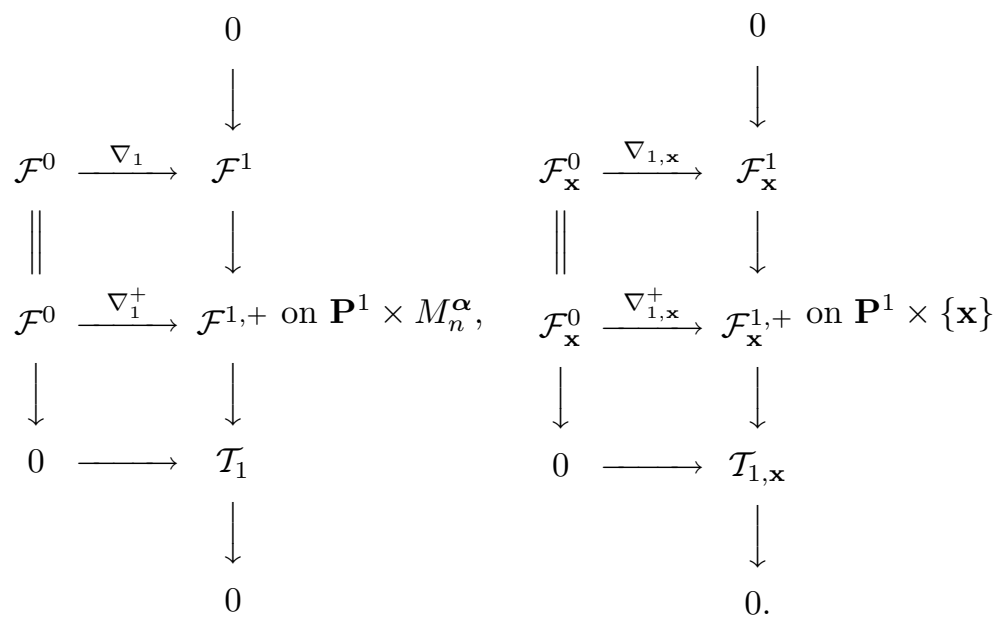

Note that at each point $t_{i}, 1 \leq i \leq n$, the stalk $\left(\mathcal{T}_{1, \mathbf{x}}\right)_{t_{i}}$ is isomorphic to $\mathbf{C}\left(\left(t_{i}, \mathbf{x}\right)\right)$, hence $H^{0}\left(\mathcal{T}_{1, \mathbf{x}}\right) \simeq \oplus_{i=1}^{n} \mathbf{C}\left(\left(t_{i}, \mathbf{x}\right)\right) \simeq \mathbf{C}^{n}$.

Lemma 6.1. At each closed point $\mathbf{x} \in M_{n}^{\boldsymbol{\alpha}}(\mathbf{t}, \boldsymbol{\lambda}) \subset M_{n}^{\boldsymbol{\alpha}}$ the tangent spaces can be given as follows.

$$
\begin{gathered}
\left(\Theta_{M_{n}^{\alpha} / T_{n}^{\prime}}\right)_{\mathbf{x}} \simeq \mathbf{H}^{1}\left(\mathbf{P}^{1},\left[\mathcal{F}_{\mathbf{x}}^{0} \stackrel{\nabla_{1, \mathbf{x}}^{+}}{\longrightarrow} \mathcal{F}_{\mathbf{x}}^{1,{ }^{+}}\right]\right), \\
\left(\Theta_{M_{n}^{\alpha} / T_{n}^{\prime} \times \Lambda_{n}}\right)_{\mathbf{x}} \simeq \mathbf{H}^{1}\left(\mathbf{P}^{1},\left[\mathcal{F}_{\mathbf{x}}^{0} \stackrel{\nabla_{1, \mathbf{x}}}{\longrightarrow} \mathcal{F}_{\mathbf{x}}^{1}\right]\right), \\
\left(\Theta_{T_{n}^{\prime} \times \Lambda_{n} / T_{n}^{\prime}}\right)_{\pi(\mathbf{x})} \simeq H^{0}\left(\mathcal{T}_{1, \mathbf{x}}\right) \simeq \mathbf{C}^{n} .
\end{gathered}
$$

Under these isomorphisms, we have the following identification of the natural exact sequences of the tangent spaces with the exact sequences of the hypercohomologies: 
(120)

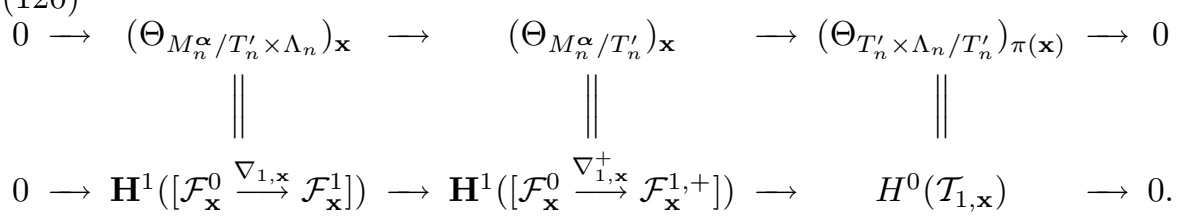

Proof. The smoothness of the natural map $\pi_{n}: M_{n}^{\alpha} \longrightarrow T_{n}^{\prime} \times \Lambda_{n}$ follows from Theorem 2.1. (Actually, one can show that $\mathbf{H}^{2}\left(\left[\mathcal{F}_{\mathbf{x}}^{0} \stackrel{\nabla_{1, \mathbf{x}}}{\longrightarrow} \mathcal{F}_{\mathbf{x}}^{1}\right]\right)=\{0\}$ (cf. Lemma 6.3)). The space of the infinitesimal deformations of logarithmic parabolic connection with fixing the eigenvalues of the residue matrix of $\tilde{\nabla}_{\mathbf{x}, t_{i}}$ at $t_{i}$ is given by the hypercohomology

$$
\mathbf{H}^{1}\left(\mathbf{P}^{1},\left[\mathcal{F}_{\mathbf{x}}^{0} \stackrel{\nabla_{1, \mathbf{x}}}{\longrightarrow} \mathcal{F}_{\mathbf{x}}^{1}\right]\right) .
$$

(Cf. Arinkin [A]). Moreover it is easy to see that $\mathbf{H}^{1}\left(\mathbf{P}^{1},\left[\mathcal{F}_{\mathbf{x}}^{0} \stackrel{\nabla_{1, \mathbf{x}}^{+}}{\longrightarrow} \mathcal{F}_{\mathbf{x}}^{1,+}\right]\right)$ is the set of infinitesimal deformations of logarithmic parabolic connections without fixing the eigenvalues of the residues of $\tilde{\nabla}_{\mathbf{x}}$.

Since $\mathcal{T}_{1, \mathbf{x}}$ is a skyscraper sheaf supported on $D(\mathbf{t}) \subset \mathbf{P}^{1} \times\{\mathbf{x}\}$, we see that $\mathbf{H}^{0}\left(0 \rightarrow \mathcal{T}_{1, \mathbf{x}}\right)=\{0\}, \mathbf{H}^{1}\left(0 \rightarrow \mathcal{T}_{1, \mathbf{x}}\right)=H^{0}\left(\mathcal{T}_{1, \mathbf{x}}\right) \simeq \mathbf{C}^{n}$. Local calculations of the maps $\nabla_{1}, \nabla_{1,+}$ in the commutative diagram in (116) show that the natural map

$$
d \pi_{n, \mathbf{x}}: \mathbf{H}^{1}\left(\left[\nabla_{1, \mathbf{x}}^{+}: \mathcal{F}_{\mathbf{x}}^{0} \longrightarrow \mathcal{F}^{1,+}\right]\right) \longrightarrow H^{0}\left(T_{1}\right)
$$

gives the differential of the map $\pi_{n}: M_{n}^{\boldsymbol{\alpha}} \longrightarrow \Lambda_{n}$ at $\mathbf{x}$. Since $\mathbf{H}^{2}\left(\left[\mathcal{F}_{\mathbf{x}}^{0} \stackrel{\nabla_{1, \mathbf{x}}}{\longrightarrow}\right.\right.$ $\left.\left.\mathcal{F}_{\mathbf{x}}^{1}\right]\right)=\{0\}$ or equivalently $\pi_{n}$ is smooth at $\mathbf{x}$, the map $d \pi_{\mathbf{x}}$ is surjective.

\section{§6.2. The relative symplectic form $\Omega$ for $\pi_{n}: M_{n}^{\alpha} \longrightarrow T_{n}^{\prime} \times \Lambda_{n}$}

Let us consider the smooth family of moduli spaces of stable parabolic connections:

$$
\pi_{n}: M_{n}^{\alpha} \longrightarrow T_{n}^{\prime} \times \Lambda_{n}
$$

Now we will show that each closed fiber $\pi_{n}^{-1}(\mathbf{t}, \boldsymbol{\lambda})=M_{n}^{\boldsymbol{\alpha}}(\mathbf{t}, \boldsymbol{\lambda}, L)$ admits a canonical symplectic structure $\Omega$, which induces a non-degenerate skew symmetric bilinear form on its tangent sheaf:

$$
\Omega_{\mid M_{n}^{\alpha}(\mathbf{t}, \boldsymbol{\lambda}, L)}: \Theta_{M_{n}^{\alpha}(\mathbf{t}, \boldsymbol{\lambda}, L)} \otimes \Theta_{M_{n}^{\alpha}(\mathbf{t}, \boldsymbol{\lambda}, L)} \longrightarrow \mathcal{O}_{M_{n}^{\alpha}(\mathbf{t}, \boldsymbol{\lambda}, L)}
$$

First, a local calculation shows the following 
Lemma 6.2. For each point $\mathbf{x} \in M_{n}^{\boldsymbol{\alpha}}(\mathbf{t}, \boldsymbol{\lambda}, L)=\pi_{n}^{-1}(\mathbf{t}, \boldsymbol{\lambda}) \subset M_{n}^{\boldsymbol{\alpha}}$, set $\mathcal{F}_{\mathbf{x}}^{i}=\mathcal{F}_{\mid \mathbf{P}^{1} \times \mathbf{x}}^{i}$ for $i=0,1$. Then we have isomorphisms

$$
\mathcal{F}_{\mathbf{x}}^{1} \simeq \mathcal{F}_{\mathbf{x}}^{0 \vee} \otimes \Omega_{\mathbf{P}^{1}}^{1}, \quad \mathcal{F}_{\mathbf{x}}^{0} \simeq \mathcal{F}_{\mathbf{x}}^{1 \vee} \otimes \Omega_{\mathbf{P}^{1}}^{1}
$$

where $\mathcal{F}_{\mathbf{x}}^{i \vee}=\mathcal{H} \operatorname{om}\left(\mathcal{F}_{\mathbf{x}}^{i}, \mathcal{O}_{\mathbf{P}^{1}}\right)$

The following lemma is a key of proof of the smoothness of the moduli space $M_{n}^{\boldsymbol{\alpha}}(\mathbf{t}, \boldsymbol{\lambda}, L)$. The stability assumption on the objects in $M_{n}^{\boldsymbol{\alpha}}(\mathbf{t}, \boldsymbol{\lambda}, L)$ is essential in this lemma.

Lemma 6.3. Under the notation as above, we have

$$
\mathbf{H}^{2}\left(\mathbf{P}^{1}, \mathcal{F}_{\mathbf{x}}^{\bullet}\right)=\{0\} .
$$

Proof. Consider the dual complex $\left(\nabla^{1}\right)^{\vee}:\left(\mathcal{F}^{1}\right)^{\vee} \otimes \Omega_{\mathbf{P}^{1}}^{1} \longrightarrow\left(\mathcal{F}^{0}\right)^{\vee} \otimes$ $\Omega_{\mathbf{P}^{1}}^{1}$ which can be identified with the original complex $\nabla^{1}$ by Killing form (cf. Lemma 6.2). Therefore

$$
\begin{aligned}
\mathbf{H}^{2}\left(\mathcal{F}^{\bullet}\right) & \simeq \operatorname{coker}\left[H^{1}\left(\mathcal{F}_{\mathbf{x}}^{0}\right) \stackrel{\nabla^{1}}{\longrightarrow} H^{1}\left(\mathcal{F}_{\mathbf{x}}^{1}\right)\right] \\
& \simeq \operatorname{ker}\left[H^{1}\left(\mathcal{F}_{\mathbf{x}}^{1}\right)^{\vee} \stackrel{\left(\nabla^{1}\right)^{\vee}}{\longrightarrow} H^{1}\left(\mathcal{F}_{\mathbf{x}}^{0}\right)^{\vee}\right]^{\vee} \\
& \simeq \operatorname{ker}\left[H^{0}\left(\mathcal{F}_{\mathbf{x}}^{0}\right) \stackrel{\nabla^{1}}{\longrightarrow} H^{0}\left(\mathcal{F}_{\mathbf{x}}^{1}\right)\right]^{\vee}
\end{aligned}
$$

Since $\mathcal{F}_{\mathrm{x}}^{0}$ is in the trace free part of the endomorphisms, it suffices to show that any $s \in H^{0}\left(\mathcal{F}_{\mathbf{x}}^{0}\right)$ such that $s \nabla=\nabla s$ is a scalar. For any $\lambda \in \mathbf{C}$, let us set $E_{\lambda}^{0}=\operatorname{ker}(s-\lambda)$ and $E_{\lambda}^{1}=\operatorname{Im}(s-\lambda)$. Then both $E_{\lambda}^{0}$ and $E_{\lambda}^{1}$ are subsheaves of $E$ stable under $\nabla$. If $E_{\lambda}^{0}$ is locally free of rank 1 , one can see that either $E_{\lambda}^{0}$ or $E_{\lambda}^{1}$ violates the stability of $E$. Hence $E_{\lambda}^{0}$ is zero or coincides with $E$. Therefore $s$ is scalar.

Proposition 6.1. There exists a global relative 2-form

$$
\Omega \in H^{0}\left(M_{n}^{\boldsymbol{\alpha}}, \Omega_{M_{n}^{\alpha} / T_{n}^{\prime} \times \Lambda_{n}}^{2}\right) .
$$

which induces a symplectic structure on each fiber of $\pi_{n}$.

Proof. Let us consider the following commutative diagram:

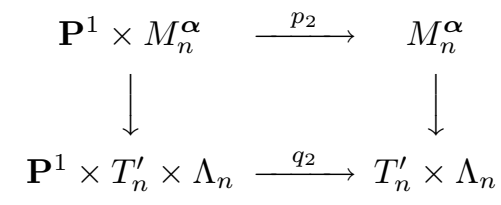


Let $\mathcal{F}^{\bullet}:=\left[\nabla_{1}: \mathcal{F}^{0} \longrightarrow \mathcal{F}^{1}\right]$ be the complex of sheaves defined in (114). From Lemma 6.1 , we have a natural isomorphism of sheaves:

$$
\mathbf{R}^{1} p_{2, *}\left(\mathcal{F}^{\bullet}\right) \stackrel{\simeq}{\longrightarrow} \Theta_{M_{n}^{\alpha} / T_{n}^{\prime} \times \Lambda_{n}} .
$$

By this isomorphism, it suffices to define a non-degenerate skew-symmetric form

$$
\Omega: \mathbf{R}^{1} p_{2, *}\left(\mathcal{F}^{\bullet}\right) \otimes \mathbf{R}^{1} p_{2, *}\left(\mathcal{F}^{\bullet}\right) \longrightarrow \mathbf{R}^{2} p_{2, *}\left(\Omega_{\mathbf{P}^{1} \times M_{n}^{\alpha} / M_{n}^{\alpha}}^{\bullet}\right) \cong \mathcal{O}_{M_{n}^{\alpha}}
$$

Let us fix a point $\mathbf{x} \in M_{n}^{\boldsymbol{\alpha}}(\mathbf{t}, \boldsymbol{\lambda}, L)=\pi_{n}^{-1}(\mathbf{t}, \boldsymbol{\lambda}) \subset M_{n}^{\boldsymbol{\alpha}}$ and define the restriction as $\mathcal{F}_{\mathbf{x}}^{\bullet}=\mathcal{F}_{\mid \mathbf{P}^{1} \times\{\mathbf{x}\}}^{\bullet}$. From the following definition of $\Omega(\mathbf{x})$ at the stalk level of (128), it is obvious the definition of the global relative 2-form $\Omega$ in (128), and the non-degeneracy of $\Omega$ will be checked at the stalk of each closed point $\mathbf{x}$.

Take an affine open covering $\left\{U_{\alpha}\right\}$ of $\mathbf{P}^{1}$ and consider the following pairing

$$
\begin{aligned}
& \Omega(\mathbf{x}): \quad \mathbf{H}^{1}\left(\mathbf{P}^{1}, \mathcal{F}_{\mathbf{x}}^{\bullet}\right) \otimes \mathbf{H}^{1}\left(\mathbf{P}^{1}, \mathcal{F}_{\mathbf{x}}^{\bullet}\right) \longrightarrow \quad \mathbf{H}^{2}\left(\Omega_{\mathbf{P}^{1}}^{\bullet}\right) \simeq \mathbf{C}(\mathbf{x}) \\
& \left(\left[\left\{v_{\alpha \beta}\right\},\left\{u_{\alpha}\right\}\right],\left[\left\{v_{\alpha \beta}^{\prime}\right\},\left\{u_{\alpha}^{\prime}\right\}\right]\right) \mapsto\left[\left\{\operatorname{Tr}\left(v_{\alpha \beta} \circ u_{\beta}^{\prime}\right)-\operatorname{Tr}\left(u_{\alpha} \circ v_{\alpha \beta}^{\prime}\right)\right\}\right] \\
& -\left[\left\{\operatorname{Tr}\left(v_{\alpha \beta} \circ v_{\beta \gamma}^{\prime}\right)\right\}\right]
\end{aligned}
$$

where we consider in Čech cohomology and $\left\{v_{\alpha \beta}\right\} \in C^{1}\left(\mathcal{F}_{\mathbf{x}}^{0}\right),\left\{u_{\alpha}\right\} \in C^{0}\left(\mathcal{F}_{\mathbf{x}}^{1}\right)$, $\left\{\tilde{\nabla}_{\mathbf{x}} v_{\alpha \beta}-v_{\alpha \beta} \tilde{\nabla}_{\mathbf{x}}\right\}=\left\{u_{\beta}-u_{\alpha}\right\}$ and so on. We can check that $\Omega(\mathbf{x})$ is a skew symmetric pairing. Let us show that $\Omega(\mathbf{x})$ is non-degenerate for any point $\mathbf{x} \in M_{n}^{\boldsymbol{\alpha}}(\mathbf{t}, \boldsymbol{\lambda}, L)$. From Lemma 6.3 , one can show that $\mathbf{H}^{2}\left(\mathcal{F}_{\mathbf{x}}^{\bullet}\right)=0$ for any $\mathbf{x} \in M_{n}^{\boldsymbol{\alpha}}(\mathbf{t}, \boldsymbol{\lambda}, L) . \Omega(\mathbf{x})$ induces a homomorphism

$$
\mathbf{H}^{1}\left(\mathcal{F}_{\mathbf{x}}^{\bullet} \stackrel{\xi}{\longrightarrow} \mathbf{H}^{1}\left(\mathcal{F}_{\mathbf{x}}^{\bullet}\right)^{\vee} .\right.
$$

From the spectral sequence $H^{p}\left(H^{q}\left(\mathcal{F}_{\mathbf{x}}^{0}\right) \rightarrow H^{q}\left(\mathcal{F}_{\mathbf{x}}^{1}\right)\right) \Rightarrow \mathbf{H}^{p+q}\left(\mathcal{F}_{\mathbf{x}}^{\bullet}\right)$, we obtain the following exact sequence

$$
0 \longrightarrow H^{0}\left(\mathcal{F}_{\mathbf{x}}^{0}\right) \longrightarrow H^{0}\left(\mathcal{F}_{\mathbf{x}}^{1}\right) \longrightarrow \mathbf{H}^{1}\left(\mathcal{F}_{\mathbf{x}}^{\bullet}\right) \longrightarrow H^{1}\left(\mathcal{F}_{\mathbf{x}}^{0}\right) \longrightarrow H^{1}\left(\mathcal{F}_{\mathbf{x}}^{1}\right) \longrightarrow 0 .
$$

Then we obtain the exact commutative diagram

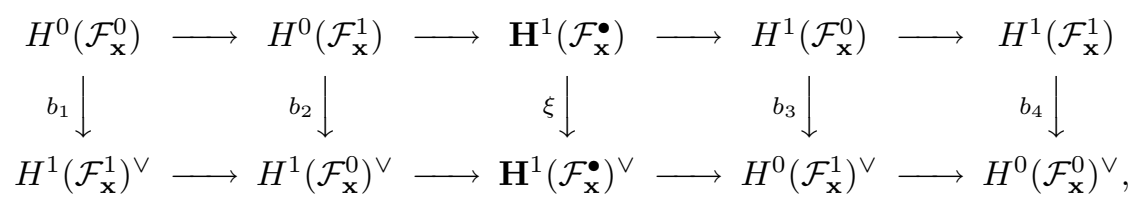


where $b_{1}, \ldots, b_{4}$ are isomorphisms induced by the isomorphisms $\mathcal{F}_{\mathbf{x}}^{0} \cong\left(\mathcal{F}^{1}\right)_{\mathbf{x}}^{\vee} \otimes$ $\Omega_{\mathbf{P}^{1}}^{1}, \mathcal{F}_{\mathbf{x}}^{1} \cong\left(\mathcal{F}^{0}\right)_{\mathbf{x}}^{\vee} \otimes \Omega_{\mathbf{P}^{1}}^{1}$ and Serre-duality. Thus $\xi$ becomes an isomorphism by five lemma.

\section{§6.3. Smoothness of $M_{n}^{\alpha}(\mathbf{t}, \boldsymbol{\lambda}, L)$ and its dimension}

In this subsection, we prove that the morphism $\pi_{n}: M_{n}^{\alpha}(L) \longrightarrow T_{n} \times \Lambda_{n}$ is smooth of equidimension $2 n-6$, which is stated in Theorem 2.1, (2).

\section{Proposition 6.2.}

(1) The morphism $\pi_{n}: M_{n}^{\alpha}(L) \longrightarrow T_{n} \times \Lambda_{n}$ is smooth.

(2) For any closed point $\mathbf{x} \in M_{n}^{\boldsymbol{\alpha}}(\mathbf{t}, \boldsymbol{\lambda}, L)$, we have

$$
\operatorname{dim}_{\mathbf{C}} \mathbf{H}^{1}\left(\mathcal{F}_{\mathbf{x}}^{\bullet}\right)=2 n-6 .
$$

In particular, the moduli space $M_{n}^{\boldsymbol{\alpha}}(\mathbf{t}, \boldsymbol{\lambda}, L)$ is smooth of equidimension $2 n-6$.

Proof. (1): By a standard argument as in [Lemma 4, [A]], the smoothness of $\pi_{n}$ at $\mathbf{x}$ follows from Lemma 6.3. (2): First, by (123), we have $\mathcal{F}^{1} \simeq\left(\mathcal{F}^{0}\right)^{\vee} \otimes$ $\Omega_{\mathbf{P}^{1}}^{1}$, and hence Serre duality implies that $\chi\left(\mathbf{P}^{1}, \mathcal{F}_{\mathbf{x}}^{1}\right)=\chi\left(\left(\mathcal{F}_{\mathbf{x}}^{0}\right)^{\vee} \otimes \Omega_{\mathbf{P}^{1}}^{1}\right)=$ $-\chi\left(\mathbf{P}^{1}, \mathcal{F}^{0}\right)$. Together with the exact sequence (130), we obtain

$$
\operatorname{dim} \mathbf{H}^{1}\left(\mathcal{F}_{\mathbf{x}}^{\bullet}\right)=-\chi\left(\mathbf{P}^{1}, \mathcal{F}_{\mathbf{x}}^{0}\right)+\chi\left(\mathbf{P}^{1}, \mathcal{F}_{\mathbf{x}}^{1}\right)=-2 \chi\left(\mathbf{P}^{1}, \mathcal{F}_{\mathbf{x}}^{0}\right)
$$

Setting $\mathcal{E} n d^{0}\left(\mathbf{E}_{\mathbf{x}}\right)=\left\{s \in \mathcal{E} n d\left(\tilde{E}_{\mathbf{x}}\right) \mid \operatorname{Tr}(s)=0\right\}$, by definition of $\mathcal{F}_{\mathbf{x}}^{0}(111)$, we obtain the following exact sequence

$$
0 \longrightarrow \mathcal{F}_{\mathbf{x}}^{0} \longrightarrow \mathcal{E} n d^{0}\left(\tilde{E}_{\mathbf{x}}\right) \longrightarrow \otimes_{i=1}^{n} \mathbf{C}\left(\left(t_{i}, \mathbf{x}\right)\right) \longrightarrow 0 .
$$

Since $\mathcal{E} n d^{0}\left(\mathbf{E}_{\mathbf{x}}\right)$ is a self-dual locally free sheaf of rank 3 on $\mathbf{P}^{1}$, Riemann-Roch theorem implies that $\chi\left(\mathbf{P}^{1}, \mathcal{E} n d^{0}\left(\mathbf{E}_{\mathbf{x}}\right)\right)=3+\operatorname{deg} \mathcal{E} n d^{0}\left(\mathbf{E}_{\mathbf{x}}\right)=3$. Then the exact sequence (133) together with (132) shows that

$$
\chi\left(\mathbf{P}^{1}, \mathcal{F}_{\mathbf{x}}^{0}\right)=\chi\left(\mathcal{E} n d^{0}\left(\tilde{E}_{\mathbf{x}}\right)\right)-n=3-n,
$$

which implies the assertion (131).

Remark 6.1. One can also show that

$$
\mathbf{H}^{2}\left(\mathbf{P}^{1}, \mathcal{F}_{\mathbf{x}}^{\bullet+}\right)=\{0\},
$$

which implies that the morphism $M_{n}^{\alpha} \longrightarrow T_{n}$ is smooth. 


\section{$\S 6.4$. Tangent space to $\mathcal{R}\left(\mathcal{P}_{n, \mathrm{t}}\right)_{\mathrm{a}}$}

Let $\left(E, \nabla, \varphi,\left\{l_{i}\right\}\right)$ be a stable parabolic connection on $\mathbf{P}^{1}$ corresponding to a point $\mathbf{x}$ in $M_{n}^{\boldsymbol{\alpha}}(\mathbf{t}, \boldsymbol{\lambda}, L)$. Let us consider the inclusion $j: \mathbf{P}^{1} \backslash D(\mathbf{t}) \hookrightarrow \mathbf{P}^{1}$ and define

$$
\mathbf{E}=\operatorname{ker}\left[\nabla^{a n}: E \longrightarrow\left(E \otimes \Omega_{\mathbf{P}^{1}}^{1}\right)\right]_{\mathbf{P}^{1} \backslash D(\mathbf{t})} .
$$

Then $\mathbf{E}$ becomes a locally constant sheaf on $\mathbf{P}^{1} \backslash D(\mathbf{t})$. The correspondence $\left(E, \nabla, \varphi,\left\{l_{i}\right\}\right) \mapsto \mathbf{E}$ induces an analytic morphism

$$
\mathbf{R H}_{\mathbf{t}, \boldsymbol{\lambda}}: M_{n}^{\boldsymbol{\alpha}}(\mathbf{t}, \boldsymbol{\lambda}, L) \longrightarrow \mathcal{R}\left(\mathcal{P}_{n, \mathbf{t}}\right)_{\mathbf{a}}
$$

which is called the Riemann-Hilbert correspondence. (Here we set $\mathbf{a}=\left(a_{i}\right), a_{i}=$ $\left.2 \cos 2 \pi \lambda_{i}\right)$. For the precise definition, see Definition 7.1 in $\S 7$.

The morphism $\mathbf{R H}_{\mathbf{t}, \boldsymbol{\lambda}}$ will be studied in detail in the next section.

Define another locally constant sheaf on $\mathbf{P}^{1} \backslash D(\mathbf{t})$ by

$$
\mathbf{V}:=\{s \in \operatorname{Hom}(\mathbf{E}, \mathbf{E}) \mid \operatorname{Tr}(s)=0\} .
$$

Note that for each point $u \in \mathbf{P}^{1} \backslash D(\mathbf{t})$ the fiber of $\mathbf{V}_{u}$ is isomorphic to the Lie algebra $s l_{2}(\mathbf{C})$. Therefore $\mathbf{V}$ admits the natural non-degenerate pairing $q$ : $\mathbf{V} \otimes \mathbf{V} \longrightarrow \mathbf{C}_{\mathbf{P}^{1} \backslash D(\mathbf{t})}$ induced by the Killing form on each fiber $\mathbf{V}_{u}, u \in \mathbf{P}^{1} \backslash D(\mathbf{t})$. Now consider the constructible sheaf $j_{*}(\mathbf{V})$ and the following exact sequence induced by the Leray spectral sequence for the inclusion $j: \mathbf{P}^{1} \backslash D(\mathbf{t}) \hookrightarrow \mathbf{P}^{1}$ :

$$
\begin{aligned}
0 \rightarrow H^{1}\left(\mathbf{P}^{1}, j_{*} \mathbf{V}\right) \rightarrow H^{1}\left(\mathbf{P}^{1} \backslash\right. & D(\mathbf{t}), \mathbf{V}) \rightarrow H^{0}\left(\mathbf{P}^{1}, R^{1} j_{*}(\mathbf{V})\right) \\
& \rightarrow H^{2}\left(\mathbf{P}^{1}, j_{*}(\mathbf{V})\right) \rightarrow H^{2}\left(\mathbf{P}^{1} \backslash D(\mathbf{t}), \mathbf{V}\right)
\end{aligned}
$$

Recall that in $\S 4$ we have obtained the morphism

$$
\phi_{n}: \mathcal{R}_{n} \longrightarrow T_{n}^{\prime} \times \mathcal{A}_{n}
$$

such that $\phi_{n}^{-1}((\mathbf{t}, \mathbf{a}))=\mathcal{R}\left(\mathcal{P}_{n, \mathbf{t}}\right)_{\mathbf{a}}$. Fixing $\mathbf{t} \in T_{n}^{\prime}$, we can also define

$$
\phi_{n, \mathbf{t}}: \mathcal{R}\left(\mathcal{P}_{n, \mathbf{t}}\right) \longrightarrow \mathbf{t} \times \mathcal{A}_{n}
$$

Lemma 6.4. Let $\left(E, \nabla, \varphi,\left\{l_{i}\right\}\right) \in M_{n}^{\boldsymbol{\alpha}}(\mathbf{t}, \boldsymbol{\lambda}, L)$ be a stable parabolic connection, and $\mathbf{E}:=\operatorname{ker} \nabla_{\mid \mathbf{P}^{1}-D(\mathbf{t})}$ the corresponding local system. Moreover let $\mathbf{V}$ be the trace free part of $\operatorname{End}(\mathbf{E})$. Let us fix a monodromy representation $\rho_{\mathbf{E}}: \pi_{1}\left(\mathbf{P}^{1} \backslash D(\mathbf{t}), *\right) \longrightarrow S L_{2}(\mathbf{C})$ associated to the local system $\mathbf{E}$. Fix canonical generators $\gamma_{i}, 1 \leq i \leq n$ of $\pi_{1}\left(\mathbf{P}^{1} \backslash D(\mathbf{t}), *\right)$ and set $M_{i}=\rho_{\mathbf{E}}\left(\gamma_{i}\right) \in S L_{2}(\mathbf{C})$ for $1 \leq i \leq n$. Consider the following conditions.

$$
\text { The representation } \rho_{\mathbf{E}} \text { is irreducible. }
$$


For each $i, 1 \leq i \leq n$, the local monodromy matrix $M_{i}$ around $t_{i}$ is not equal to $\pm I_{2}$.

(1) Under the condition (141), we have

$$
H^{2}\left(\mathbf{P}^{1} \backslash D(\mathbf{t}), \mathbf{V}\right)=\{0\} .
$$

(2) Under the conditions (141) and (142), we have a sheaf isomorphism

$$
R^{1} j_{*}(\mathbf{V}) \simeq \oplus_{i=1}^{n} \mathbf{C}\left(t_{i}\right)
$$

and the exact sequence of cohomology groups.

$$
0 \longrightarrow H^{1}\left(\mathbf{P}^{1}, j_{*} \mathbf{V}\right) \longrightarrow H^{1}\left(\mathbf{P}^{1} \backslash D(\mathbf{t}), \mathbf{V}\right) \longrightarrow H^{0}\left(\mathbf{P}^{1}, R^{1} j_{*}(\mathbf{V})\right) \longrightarrow 0 .
$$

Proof. Since we have a canonical non-degenerate pairing

$$
j_{*}(\mathbf{V}) \otimes j_{*}(\mathbf{V}) \stackrel{\text { Killing }}{\longrightarrow} \mathbf{C}_{\mathbf{P}^{1}}
$$

we have a self-duality $\left(j_{*} \mathbf{V}\right)^{\vee} \simeq j_{*} \mathbf{V}$ and hence a duality isomorphism

$$
H^{2}\left(\mathbf{P}^{1}, j_{*}(\mathbf{V})\right) \simeq H^{0}\left(\mathbf{P}^{1}, j_{*} \mathbf{V}\right)^{\vee} \simeq H^{0}\left(\mathbf{P}^{1} \backslash D(\mathbf{t}), \mathbf{V}\right)^{\vee}
$$

Since by (141) the monodromy representation $\rho_{\mathbf{E}}$ is irreducible, $H^{0}\left(\mathbf{P}^{1} \backslash D(\mathbf{t})\right.$, $\operatorname{End}(\mathbf{E})) \simeq \mathbf{C} \cdot I d_{\mathbf{E}}$ by Schur's lemma and hence its trace free part $H^{0}\left(\mathbf{P}^{1} \backslash\right.$ $D(\mathbf{t}), \mathbf{V})$ is $\{0\}$, thus

$$
H^{2}\left(\mathbf{P}^{1}, j_{*}(\mathbf{V})\right)=\{0\}
$$

Moreover $H^{1}\left(\mathbf{P}^{1}, R^{1} j_{*} \mathbf{V}\right)=\{0\}$, for the sheaf $R^{1} j_{*} \mathbf{V}$ is supported only on $D(\mathbf{t})=t_{1}+\cdots+t_{n}$. Then the assertion (143) now easily follows from the Leray spectral sequence for $j: \mathbf{P}^{1} \backslash D(\mathbf{t}) \hookrightarrow \mathbf{P}^{1}$.

From (138), we obtain the exact sequence (145) because of (146).

For the assertion (144), we first remark that the sheaf $R^{1} j_{*} \mathbf{V}$ is supported on $D(\mathbf{t})=t_{1}+\cdots+t_{n}$. We will determine the stalk $R^{1} j_{*} \mathbf{V}_{t_{i}}$ at each $t_{i}$. Let us take a small neighborhood $U_{i}$ of $t_{i}$ and $u_{i} \in U_{i}-\left\{t_{i}\right\}$. Then one can identify the fiber $\mathbf{V}_{u_{i}}$ with the symmetric tensor $\operatorname{Sym}^{2}\left(\mathbf{E}_{u_{i}}\right)$. Consider the $\mathbf{V}_{u_{i}} \simeq \operatorname{Sym}^{2}\left(\mathbf{E}_{u_{i}}\right)$ as the vector space with the action of $M_{i}$. Then define the invariant part as 


$$
\begin{aligned}
& \mathbf{V}_{u_{i}}^{\left\langle M_{i}\right\rangle}=\operatorname{Sym}^{2}\left(\mathbf{E}_{u_{i}}\right)^{\left\langle M_{i}\right\rangle} \\
&:=\operatorname{ker}\left(\operatorname{Sym}^{2}\left(M_{i}\right)-I d: \operatorname{Sym}^{2}\left(\mathbf{E}_{u_{i}}\right) \rightarrow \operatorname{Sym}^{2}\left(\mathbf{E}_{u_{i}}\right)\right) .
\end{aligned}
$$

Then it is easy to see that

$$
R^{1} j_{*} \mathbf{V}_{t_{i}} \simeq\left(\mathbf{V}_{u_{i}}^{\left\langle M_{i}\right\rangle}\right)^{\vee}
$$

Choose a suitable basis of $\mathbf{E}_{u_{i}}$ and write $M_{i}$ as $M_{i}=\left(\begin{array}{ll}a & b \\ c & d\end{array}\right)$ with $a d-b c=1$. Then the action of $M_{i}$ on $\operatorname{Sym}^{2}\left(\mathbf{E}_{u_{i}}\right)$ has the following matrix representation.

$$
\operatorname{Sym}^{2}\left(M_{i}\right):=\left(\begin{array}{ccc}
a^{2} & a b & b^{2} \\
2 a c & a d+b c & 2 b d \\
c^{2} & c d & d^{2}
\end{array}\right)
$$

Then it is easy to check that the eigenvalues of $S y m^{2}\left(M_{i}\right)$ are given by the roots of

$$
(x-1)\left(x^{2}-\left((a+d)^{2}-2\right) x+1\right)=0 .
$$

If neither of the roots of $x^{2}-\left((a+d)^{2}-2\right) x+1=0$ is 1 , then $\operatorname{dim} \operatorname{ker}\left(\operatorname{Sym}^{2}\left(M_{i}\right)-\right.$ $I d)=1$. If one of the roots of $x^{2}-\left((a+d)^{2}-2\right) x+1=0$ is one, then we have $(a+d)^{2}=4$, which implies that $a+d= \pm 2$. For those cases, the eigenvalues of $M_{i}$ are 1 or -1 respectively. We may assume that $M_{i} \neq \pm I_{2}$. Then we can assume that $M_{i}=\left(\begin{array}{ll}1 & b \\ 0 & 1\end{array}\right)$ or $M_{i}=\left(\begin{array}{cc}-1 & b \\ 0 & -1\end{array}\right)$ with $b \neq 0$. For these cases, we can write

$$
\operatorname{Sym}^{2}\left(M_{i}\right)=\left(\begin{array}{ccc}
1 & b & b^{2} \\
0 & 1 & 2 b \\
0 & 0 & 1
\end{array}\right) \quad \text { or } \quad\left(\begin{array}{ccc}
1 & -b & b^{2} \\
0 & 1 & -2 b \\
0 & 0 & 1
\end{array}\right) \text {. }
$$

Now it is easy to check that $\operatorname{dim} \operatorname{ker}\left(\operatorname{Sym}^{2}\left(M_{i}\right)-I d\right)=1$.

Lemma 6.5. Let us fix $\mathbf{t} \in T_{n}^{\prime}$. The notation being as in Lemma 6.4, let us take a point $\mathbf{y}:=[\mathbf{E}] \in \mathcal{R}\left(\mathcal{P}_{n, \mathbf{t}}\right)_{\mathbf{a}} \subset \mathcal{R}\left(\mathcal{P}_{n, \mathbf{t}}\right)$.

(1) Assume that the condition (141) holds for $\mathbf{E}$. Then the total space $\mathcal{R}\left(\mathcal{P}_{n, \mathbf{t}}\right)$ is smooth at $\mathbf{y}=[\mathbf{E}]$ and we have the isomorphism

$$
\Theta_{\mathcal{R}\left(\mathcal{P}_{n, \mathbf{t}}\right), \mathbf{y}} \simeq H^{1}\left(\mathbf{P}^{1} \backslash D(\mathbf{t}), \mathbf{V}\right) .
$$

(2) Assume that the conditions (141) and (142) hold for $\mathbf{E}$. Then, the map $\phi_{n, \mathbf{t}}: \mathcal{R}\left(\mathcal{P}_{n, \mathbf{t}}\right) \longrightarrow \mathbf{t} \times \mathcal{A}_{n}$ is also smooth at $\mathbf{y}=[\mathbf{E}]$. Hence the fiber $\phi_{n, \mathbf{t}}^{-1}(\mathbf{a})=\mathcal{R}\left(\mathcal{P}_{n, \mathbf{t}}\right)_{\mathbf{a}}$ is smooth at $\mathbf{y}$ where $\mathbf{a}=\phi_{n, \mathbf{t}}(\mathbf{y})$. Moreover we have the following linear isomorphisms:

$$
\left(\Theta_{\mathcal{R}\left(\mathcal{P}_{n, \mathbf{t}}\right)_{\mathbf{a}}}\right)_{\mathbf{y}} \simeq H^{1}\left(\mathbf{P}^{1}, j_{*} \mathbf{V}\right)
$$




$$
\begin{aligned}
\left(\Theta_{\mathcal{R}\left(\mathcal{P}_{n, \mathbf{t}}\right)}\right)_{\mathbf{y}} & \simeq H^{1}\left(\mathbf{P}^{1} \backslash D(\mathbf{t}), \mathbf{V}\right) \\
\left(\Theta_{\mathbf{t} \times \mathcal{A}_{n}}\right)_{\phi_{n, \mathbf{t}}(\mathbf{y})} & \simeq H^{0}\left(\mathbf{P}^{1}, R^{1} j_{*}(\mathbf{V})\right)
\end{aligned}
$$

Under the isomorphisms above, we have the following identification of the natural exact sequences of the tangent spaces with the sequence (145)

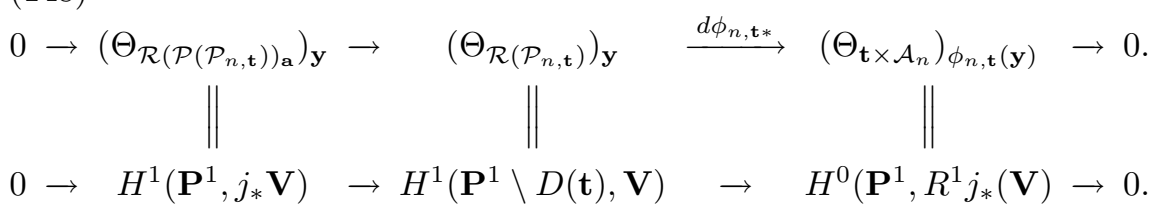

Proof. 1. Since $\mathbf{E}$ is irreducible, it is easy to see that the Zariski tangent space $\Theta_{\mathcal{R}\left(\mathcal{P}_{n, \mathbf{t}}\right), \mathbf{y}}$ of $\mathcal{R}\left(\mathcal{P}_{n, \mathbf{t}}\right)$ at $\mathbf{y}=[\mathbf{E}]$ is given by $H^{1}\left(\mathbf{P}^{1} \backslash D(\mathbf{t}), \mathbf{V}\right)$ and the obstructions to deformations lie in $H^{2}(\mathbf{P} \backslash D(\mathbf{t}), \mathbf{V})$. Since we assume that $\rho_{\mathbf{E}}$ is irreducible, we have $H^{2}(\mathbf{P} \backslash D(\mathbf{t}), \mathbf{V})=\{0\}$ (cf. (143)), from which the assertion follows.

2. From Lemma 6.4, under the assumptions, we can see that the differential $\left(\Theta_{\mathcal{R}\left(\mathcal{P}_{n, \mathbf{t}}\right)}\right)_{\mathbf{y}} \stackrel{d \phi_{n, \mathbf{t} *}}{\longrightarrow}\left(\Theta_{\mathbf{t} \times \mathcal{A}_{n}}\right)_{\phi_{n, \mathbf{t}}(\mathbf{y})}$ can be identified with the linear map

$$
H^{1}\left(\mathbf{P}^{1} \backslash D(\mathbf{t}), \mathbf{V}\right) \longrightarrow H^{0}\left(\mathbf{P}^{1}, R^{1} j_{*}(\mathbf{V})\right) \simeq \mathbf{C}^{n},
$$

which is surjective because of $H^{2}\left(\mathbf{P}^{1}, j_{*} \mathbf{V}\right)=\{0\}$. Therefore the map $\phi_{n, \mathbf{t}}$ is smooth at $\mathbf{y}=[\mathbf{E}]$ and the fiber $\phi_{n, \mathbf{t}}^{-1}(\mathbf{a})=\mathcal{R}\left(\mathcal{P}_{n, \mathbf{t}}\right)_{\mathbf{a}}$ is smooth at $\mathbf{y}$. Other assertions now easily follow from the exact sequence (145).

Lemma 6.6. Under the conditions (141) and (142) for $\mathbf{E}$, we have an isomorphism of locally constant sheaves

$$
j_{*} \mathbf{V} \simeq \operatorname{ker} \nabla_{1} \simeq\left[\nabla_{1}: \mathcal{F}^{0} \longrightarrow \mathcal{F}^{1}\right]
$$

which induces a canonical isomorphism

$$
H^{1}\left(\mathbf{P}^{1}, j_{*} \mathbf{V}\right) \stackrel{\simeq}{\longrightarrow} \mathbf{H}^{1}\left(\left[\nabla_{1}: \mathcal{F}^{0} \longrightarrow \mathcal{F}^{1}\right]\right) .
$$

Moreover we have the canonical non-degenerate pairing

$$
H^{1}\left(\mathbf{P}^{1}, j_{*} \mathbf{V}\right) \otimes H^{1}\left(\mathbf{P}^{1}, j_{*} \mathbf{V}\right) \longrightarrow H^{2}\left(\mathbf{P}^{1}, \mathbf{C}_{\mathbf{P}^{1}}\right) \simeq \mathbf{C},
$$

which induces the non-degenerate pairing $\Omega_{1}(\mathbf{y})$ at $\mathbf{y}=\mathbf{E}$

$$
\Omega_{1}(\mathbf{y}):\left(\Theta_{\mathcal{R}\left(\mathcal{P}_{n, \mathbf{t}}\right)_{\mathbf{a}}}\right)_{\mathbf{y}} \otimes\left(\Theta_{\mathcal{R}\left(\mathcal{P}_{n, \mathbf{t}}\right)_{\mathbf{a}}}\right)_{\mathbf{y}} \longrightarrow\left(\mathcal{O}_{\mathcal{R}\left(\mathcal{P}_{n, \mathbf{t}}\right)_{\mathbf{a}}}\right)_{\mathbf{y}} .
$$

This pairing can be identified with (129) via the isomorphism (150). 
Proof. The assertion (149) is trivial at the point $u \in \mathbf{P}^{1} \backslash D(\mathbf{t})$. At each point $t_{i} i=1, \ldots, n$, we will describe the connection $\nabla$ and $\nabla_{1}$ locally around $t_{i}$. Let us set $n=n_{i}=\operatorname{res}_{t_{i}}\left(\nabla_{L}\right) \in \mathbf{Z}$. We separate the proof into two cases.

i) Let $\lambda, n-\lambda$ be the eigenvalues of $\operatorname{res}_{t_{i}}(\nabla)$. First assume that $2 \lambda \notin \mathbf{Z}$. Then $\lambda \neq n-\lambda$. By a standard reduction theory of connection near a regular singularity, we can choose a suitable local coordinate $z$ around $t=t_{i}$ and write down the connection matrix of $\nabla$ by

$$
\nabla=\frac{d z}{z-t}\left(\begin{array}{cc}
\lambda & 0 \\
0 & n-\lambda
\end{array}\right)
$$

Then for a local section $s=\left(\begin{array}{cc}s_{1} & s_{2} \\ s_{3} & -s_{1}\end{array}\right) \in \operatorname{End}(E)$, the connection $\nabla_{1} s=\nabla_{E} s-$ $s \nabla_{E}$ is given by

$$
\left(\begin{array}{cc}
s_{1} & s_{2} \\
s_{3} & -s_{1}
\end{array}\right) \mapsto\left(\begin{array}{cc}
d s_{1} & d s_{2}+(2 \lambda-n) s_{2}(z-t)^{-1} d z \\
d s_{3}+(n-2 \lambda) s_{3}(z-t)^{-1} d z & -d s_{1}
\end{array}\right) .
$$

Solving $\nabla_{1}=0$ locally near $z=t$, we obtain the local solutions for $z \neq t$ as follows.

$$
c_{1}\left(\begin{array}{cc}
1 & 0 \\
0 & -1
\end{array}\right)+c_{2}\left(\begin{array}{cc}
0 & (z-t)^{n-2 \lambda} \\
0 & 0
\end{array}\right)+c_{3}\left(\begin{array}{cc}
0 & 0 \\
(z-t)^{2 \lambda-n} & 0
\end{array}\right) .
$$

Here, $c_{1}, c_{2}, c_{3} \in \mathbf{C}$ are constants. These solutions have to be single-valued well-defined section around $z=t$, hence ker $\nabla_{1}$ is generated by $\left(\begin{array}{cc}1 & 0 \\ 0 & -1\end{array}\right)$. (Note that this local section lies in $\mathcal{F}^{0}$.) On the other hand, the stalk $\left(j_{*} \mathbf{V}\right)_{t}$ is the space of monodromy invariant trace-free endomorphisms of $\mathbf{E}_{u}$, which is also generated by $\left(\begin{array}{cc}1 & 0 \\ 0 & -1\end{array}\right)$. Hence we have an isomorphism $\left(j_{*} \mathbf{V}\right)_{t} \simeq \operatorname{ker}\left(\nabla_{1}\right)_{t}$.

ii) Again, let $\lambda, n-\lambda$ be the eigenvalues of $\operatorname{res}_{t_{i}}(\nabla)$ and assume that $2 \lambda \in \mathbf{Z}$. Since we assume that the local monodromy $M_{i}$ is not $\pm I_{2}$, by a reduction theory of a connection near a regular singularity, we can choose a suitable local coordinate $z$ around $t=t_{i}$ and write down the connection matrix of $\nabla$ by

$$
\nabla=\frac{d z}{z-t}\left(\begin{array}{cc}
m_{1} & (z-t)^{m_{2}-m_{1}} \\
0 & m_{2}
\end{array}\right)
$$

where $2 m_{1}, 2 m_{2} \in \mathbf{Z}, m_{2}-m_{1} \in \mathbf{Z}$ and $m_{1} \leq m_{2}$. For local section $\left(\begin{array}{cc}s_{1} & s_{2} \\ s_{3} & -s_{1}\end{array}\right) \in$ $\operatorname{End}(E)$, the connection $\nabla_{1} s$ can be given by

$$
\begin{aligned}
& \left(\begin{array}{cc}
s_{1} & s_{2} \\
s_{3} & -s_{1}
\end{array}\right) \mapsto \\
& \left(\begin{array}{cc}
d s_{1}+s_{3}(z-t)^{m_{2}-m_{1}-1} d z & d s_{2}-2 s_{1}(z-t)^{m_{2}-m_{1}-1} d z+s_{2}\left(m_{1}-m_{2}\right)(z-t)^{-1} d z \\
d s_{3}+s_{3}\left(m_{2}-m_{1}\right)(z-t)^{-1} d z & -d s_{1}-s_{3}(z-t)^{m_{2}-m_{1}-1} d z
\end{array}\right) .
\end{aligned}
$$


Solving $\nabla_{1} s=0$ locally for $z \neq t$, we obtain the solutions

$$
\begin{aligned}
s= & c_{1}\left(\begin{array}{cc}
0 & (z-t)^{m_{2}-m_{1}} \\
0 & 0
\end{array}\right)+c_{2}\left(\begin{array}{cc}
1 & 2(z-t)^{m_{2}-m_{1}} \log (z-t) \\
0 & -1
\end{array}\right) \\
& +c_{3}\left(\begin{array}{cc}
\log (z-t) & (z-t)^{m_{2}-m_{1}}(\log (z-t))^{2} \\
-(z-t)^{m_{1}-m_{2}} & -\log (z-t)
\end{array}\right)
\end{aligned}
$$

where $c_{1}, c_{2}, c_{3} \in \mathbf{C}$ are the constants. Then we can see that all single valued solutions for $\operatorname{ker} \nabla_{1}$ are

$$
c_{1}\left(\begin{array}{cc}
0 & (z-t)^{m_{2}-m_{1}} \\
0 & 0
\end{array}\right),
$$

which are also sections of $\left(j_{*} \mathbf{V}\right)_{t}$. Therefore we have an isomorphism $\left(\operatorname{ker} \nabla_{1}\right)_{t} \simeq\left(j_{*} \mathbf{V}\right)_{t}$. Hence we have proved the assertion (149) which shows also (150).

It is easy to see that the pairing of sheaves $j_{*} \mathbf{V} \otimes j_{*} \mathbf{V} \longrightarrow j_{*} \mathbf{C}_{\mathbf{P}^{1} \backslash D(\mathbf{t})} \simeq$ $\mathbf{C}_{\mathbf{P}^{1}}$ is non-degenerate at each point of $\mathbf{P}^{1}$. Therefore, the pairing (151) is also non-degenerate.

Summarizing all results in this section, we have the following

Proposition 6.3. Let $\phi_{n}: \mathcal{R}_{n} \longrightarrow T_{n}^{\prime} \times \mathcal{A}_{n}$ be a family of moduli spaces of representations of the fundamental group $\pi_{1}\left(\mathbf{P}^{1} \backslash D(\mathbf{t}), *\right)$ as in (139). Let $\mathcal{R}_{n}^{\sharp}$ be the subset of $\mathcal{R}_{n}$ whose closed points satisfy the conditions (141) and (142).

Then $\mathcal{R}_{n}^{\sharp}$ is a non-singular variety and the restricted morphism

$$
\phi_{n}: \mathcal{R}_{n}^{\sharp} \longrightarrow T_{n}^{\prime} \times \mathcal{A}_{n}
$$

is smooth, so that all fibers $\mathcal{R}_{n,(\mathbf{t}, \mathbf{a})}^{\sharp}=\mathcal{R}\left(\mathcal{P}_{n, \mathbf{t}}\right)_{\mathbf{a}}^{\sharp}$ are non-singular varieties. On $\mathcal{R}_{n}^{\sharp}$, there exists a relative symplectic form

$$
\Omega_{1} \in \Gamma\left(\mathcal{R}_{n}^{\sharp}, \Omega_{\mathcal{R}_{n}^{\sharp} / T_{n}^{\prime} \times \mathcal{A}_{n}}^{2}\right)
$$

induced by (152).

Remark 6.2.

(1) Since $p_{2} \circ \phi_{n}: \mathcal{R}_{n}^{\sharp} \longrightarrow T_{n}^{\prime} \times \mathcal{A}_{n} \longrightarrow T_{n}^{\prime}$ is locally trivial, one can lift $\Omega_{1} \in \Gamma\left(\mathcal{R}_{n}^{\sharp}, \Omega_{\mathcal{R}_{n}^{\sharp} / T_{n}^{\prime} \times \mathcal{A}_{n}}^{2}\right)$ to a relative regular 2 -form

$$
\tilde{\Omega}_{1} \in \Gamma\left(\mathcal{R}_{n}^{\sharp}, \Omega_{\mathcal{R}_{n}^{\prime \sharp} / \mathcal{A}_{n}}^{2}\right) .
$$


In $\S 7$, we can define the Riemann-Hilbert correspondence $\mathbf{R H}_{n}: M_{n}^{\boldsymbol{\alpha}} \longrightarrow$ $\mathcal{R}_{n}$ which is a surjective holomorphic map. Set $\left(M_{n}^{\boldsymbol{\alpha}}\right)^{\sharp}=\mathbf{R H}_{n}^{-1}\left(\mathcal{R}_{n}^{\sharp}\right)$. From Lemma 6.6, one can see that $\mathbf{R H}_{n \mid \mathcal{R}_{n}^{\sharp}}^{*}\left(\Omega_{1}\right)$ coincides with the two form $\Omega_{\mid\left(M_{n}^{\boldsymbol{\alpha}}\right)^{\sharp}} \in \Gamma\left(\left(M_{n}^{\boldsymbol{\alpha}}\right)^{\sharp}, \Omega_{\left(M_{n}^{\alpha}\right)^{\sharp} / T_{n}^{\prime} \times \Lambda_{n}}^{2}\right)$ defined in (129). Pulling back $\tilde{\Omega}_{1}$ via $\mathbf{R H}_{n \mid \mathcal{R}_{n}^{\sharp}}$, we obtain

$$
\tilde{\Omega} \in \Gamma\left(\left(M_{n}^{\boldsymbol{\alpha}}\right)^{\sharp}, \Omega_{\left(M_{n}^{\alpha}\right)^{\sharp} / \Lambda_{n}}^{2}\right)
$$

which is a lift of $\Omega_{\mid\left(M_{n}^{\alpha}\right)^{\sharp}}$ via the canonical morphism $\Gamma\left(\left(M_{n}^{\alpha}\right)^{\sharp}, \Omega_{\left(M_{n}^{\alpha}\right)^{\sharp} / \Lambda_{n}}^{2}\right)$ $\longrightarrow \Gamma\left(\left(M_{n}^{\boldsymbol{\alpha}}\right)^{\sharp}, \Omega_{\left(M_{n}^{\alpha}\right)^{\sharp} / T_{n}^{\prime} \times \Lambda_{n}}^{2}\right)$. (Note that a lift $\tilde{\Omega}$ can be induced from the splitting homomorphism (20) and the splitting homomorphism can be defined algebraically). Since the codimension of $M_{n}^{\boldsymbol{\alpha}} \backslash\left(M_{n}^{\boldsymbol{\alpha}}\right)^{\sharp}$ in $M_{n}^{\boldsymbol{\alpha}}$ is at least two, the two form $\tilde{\Omega}$ can be extended to a regular relative two form on $M_{n}^{\alpha}$ which will be denoted also by $\tilde{\Omega}$. This extended two form $\tilde{\Omega} \in \Gamma\left(M_{n}^{\boldsymbol{\alpha}}, \Omega_{M_{n}^{\boldsymbol{\alpha}} / \Lambda_{n}}^{2}\right)$ is a lift of $\Omega \in \Gamma\left(M_{n}^{\boldsymbol{\alpha}}, \Omega_{M_{n}^{\boldsymbol{\alpha}} / T_{n}^{\prime} \times \Lambda_{n}}^{2}\right)$ in (129) on the whole total space $M_{n}^{\alpha}$.

(2) The closedness of $\tilde{\Omega},\left(d_{M_{n}^{\alpha} / \Lambda_{n}}(\tilde{\Omega})=0\right)$, can be proved as follows. It is easy to see that the two form $\tilde{\Omega}$ here coincides with the symplectic two form introduced in [Iw1] and [Iw2] on a Zariski dense open subset $\left(M_{n}^{\alpha}\right)^{\prime}$ of $M_{n}^{\alpha}$. As proved in [Iw1], [Iw2], there exists a suitable affine open covering $\left\{U_{i}\right\}_{i}$ of $\left(M_{n}^{\boldsymbol{\alpha}}\right)^{\prime}$ with local coordinates (for $U_{i}$ )

$$
\left(q_{1}^{i}, \ldots, q_{r}^{i}, p_{1}^{i}, \ldots, p_{r}^{i}, t_{1}, \ldots, t_{n}, \lambda_{1}, \ldots, \lambda_{n}\right)
$$

such that $\tilde{\Omega}_{\mid U_{i}}$ can be written as

$$
\tilde{\Omega}_{\mid U_{i}}=\sum_{k=1}^{r} d q_{k}^{i} \wedge d p_{k}^{i}-\sum_{l=1}^{n} d t_{l} \wedge d H_{l}^{i}(\mathbf{p}, \mathbf{q}, \mathbf{t}, \boldsymbol{\lambda}) .
$$

where $r=n-3$ (= the half of the relative dimension of $\pi_{n}$ ) and $H_{l}^{i}(\mathbf{p}, \mathbf{q}, \mathbf{t}, \boldsymbol{\lambda})$ are regular algebraic functions on $U_{i}$. The closedness $d_{M_{n}^{\alpha} / \Lambda_{n}}(\tilde{\Omega})=0$ on $U_{i}$ easily follows from the expression (157), hence by analytic continuation we see that $d_{M_{n}^{\alpha} / \Lambda_{n}}(\tilde{\Omega})=0$ on the total space $M_{n}^{\alpha}$.

(3) The regular functions $H_{l}^{i}(\mathbf{p}, \mathbf{q}, \mathbf{t}, \boldsymbol{\lambda})$ on $U_{i}$ in (157) are called Hamiltonians for Painlevé or Garnier systems with respect to the time variable $t_{l}$. Actually on an affine open set $U_{i}$ one can obtain the Hamiltonian systems (Cf. [Iw1], [Iw2]).

$$
\frac{\partial q_{k}^{i}}{\partial t_{l}}=\frac{\partial H_{l}^{i}}{\partial p_{k}^{i}}, \quad \frac{\partial p_{k}^{i}}{\partial t_{l}}=-\frac{\partial H_{l}^{i}}{\partial q_{k}^{i}} \quad(1 \leq k \leq n-3,1 \leq l \leq n) .
$$


Although these Hamiltonian systems are defined on a Zariski open subset $\left(M_{n}^{\alpha}\right)^{\prime}$ of $M_{n}^{\alpha}$, these Hamiltonian systems can be extended to Hamiltonian systems on the total space $M_{n}^{\alpha}$. This is because global vector fields on $M_{n}^{\alpha}$ induced from the isomonodromic flows coincide with these Hamiltonian systems on the Zariski open set $\left(M_{n}^{\boldsymbol{\alpha}}\right)^{\prime}$ and the global vector fields on $M_{n}^{\boldsymbol{\alpha}}$ also preserves the symplectic form $\tilde{\Omega}$.

\section{$\S 7 . \quad$ The Riemann-Hilbert Correspondence}

In this section, we also work over $T_{n}^{\prime}$ (cf. (94)). Fix $(\mathbf{t}, \boldsymbol{\lambda}) \in T_{n}^{\prime} \times \Lambda_{n}$ and set $D(\mathbf{t})=t_{1}+\cdots+t_{n} \subset \mathbf{P}^{1}, a_{i}=2 \cos 2 \pi \lambda_{i}$ and $\mathbf{a}=\left(a_{1}, \ldots, a_{n}\right) \in \mathcal{A}_{n}$. Moreover fix a determinant line bundle $L=\left(L, \nabla_{L}\right)$ on $\mathbf{P}^{1}$ such that $\operatorname{res}_{t_{i}}\left(\nabla_{L}\right) \in \mathbf{Z}$ for every $1 \leq i \leq n$. We have defined two moduli spaces $M_{n}^{\boldsymbol{\alpha}}(\mathbf{t}, \boldsymbol{\lambda}, L)$ in (42) and $\mathcal{R}\left(\mathcal{P}_{n, \mathbf{t}}\right)_{\mathbf{a}}$ in (97). In this section, we define the Riemann-Hilbert correspondence $\mathbf{R H}_{\mathbf{t}, \boldsymbol{\lambda}}: M^{\boldsymbol{\alpha}}(\mathbf{t}, \boldsymbol{\lambda}, L) \longrightarrow \mathcal{R}\left(\mathcal{P}_{n, \mathbf{t}}\right)_{\mathbf{a}}$, and show our main results for the RiemannHilbert correspondence (Theorem 7.1).

\section{§7.1. Definition of $\mathbf{R H}_{t, \lambda}$}

As in (136), take $E=(E, \nabla, \varphi, l) \in M_{n}^{\boldsymbol{\alpha}}(\mathbf{t}, \boldsymbol{\lambda}, L)$ and define the local system on $\mathbf{P}^{1} \backslash D(\mathbf{t})$ as $\mathbf{E}=\operatorname{ker}\left(\nabla_{\mid \mathbf{P}^{1} \backslash D(\mathbf{t})}\right)^{\text {an }}$. (Here we denote by $\left(\nabla_{\mid \mathbf{P}^{1} \backslash D(\mathbf{t})}\right)^{\text {an }}$ the analytic connection associated to $\left(\nabla_{\mid \mathbf{P}^{1} \backslash D(\mathbf{t})}\right)$.) Choosing a suitable flat basis for the fiber $\mathbf{E}_{*}$ at the base point $* \in \mathbf{P}^{1} \backslash D(\mathbf{t})$, one can define a monodromy representation $\rho_{\mathbf{E}}: \pi^{1}\left(\mathbf{P}^{1} \backslash D(\mathbf{t}), *\right) \longrightarrow S L_{2}(\mathbf{C})$. The difference of choices of flat basis can be given by the adjoint action of $S L_{2}(\mathbf{C})$, and hence one has a correspondence

$$
E=(E, \nabla, \varphi, l) \mapsto\left[\rho_{\mathbf{E}}\right]
$$

Here $\left[\rho_{\mathbf{E}}\right]$ denotes the Jordan equivalence class of $\rho_{\mathbf{E}}$.

Fix canonical generators $\gamma_{i}, 1 \leq i \leq n$ of $\pi^{1}\left(\mathbf{P}^{1} \backslash D(\mathbf{t}), *\right)$. For a monodromy representation $\rho_{\mathbf{E}}$ of $(E, \nabla, \varphi, l)$, set $M_{i}=\rho_{\mathbf{E}}\left(\gamma_{i}\right)$ as in $\S 4$. Since eigenvalues of $\operatorname{res}_{t_{i}}(\nabla)$ can be given by $\lambda_{i}, \operatorname{res}_{t_{i}}\left(\nabla_{L}\right)-\lambda_{i}$ and $\operatorname{res}_{t_{i}}\left(\nabla_{L}\right) \in \mathbf{Z}$, we see that the eigenvalues of $M_{i}$ are given by $\exp \left(\mp 2 \pi \sqrt{-1} \lambda_{i}\right)$. Therefore, we have local exponents for $\rho_{\mathbf{E}}$

$$
a_{i}:=\operatorname{Tr}\left[M_{i}\right]=\exp \left(-2 \pi \sqrt{-1} \lambda_{i}\right)+\exp \left(2 \pi \sqrt{-1} \lambda_{i}\right)=2 \cos \left(2 \pi \lambda_{i}\right)
$$

which are invariant under the adjoint action. 
Definition 7.1. Under the relation (160), the correspondence (159) gives an analytic morphism

$$
\mathbf{R H}_{\mathbf{t}, \boldsymbol{\lambda}}: M_{n}^{\boldsymbol{\alpha}}(\mathbf{t}, \boldsymbol{\lambda}, L) \longrightarrow \mathcal{R}\left(\mathcal{P}_{n, \mathbf{t}}\right)_{\mathbf{a}},
$$

which is called the Riemann-Hilbert correspondence.

\section{$\S 7.2$. Fundamental properties of Riemann-Hilbert correspondence}

Let us assume that $n \geq 4$. In $\S 4$, (96), we have defined the family of moduli spaces of representations of fundamental group $\phi_{n}: \mathcal{R}_{n} \longrightarrow T_{n}^{\prime} \times \mathcal{A}_{n}$ and we also have a smooth family $\pi_{n}: M_{n}^{\alpha}(L) \longrightarrow T_{n}^{\prime} \times \Lambda_{n}$ whose geometric fibers are $M^{\boldsymbol{\alpha}}(\mathbf{t}, \boldsymbol{\lambda}, L)$ (cf. Theorem 2.1). From Definition 7.1 we obtain the following commutative diagram:

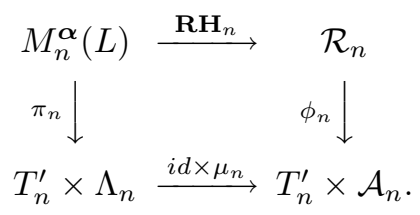

Here $\mu_{n}: \Lambda_{n} \longrightarrow \mathcal{A}_{n}$ is given by

$$
\mu_{n}\left(\lambda_{1}, \ldots, \lambda_{n}\right)=\left(a_{1}, \ldots, a_{n}\right)=\left(2 \cos \left(2 \pi \lambda_{1}\right), \ldots, 2 \cos \left(2 \pi \lambda_{n}\right)\right) .
$$

Of course, for each $(\mathbf{t}, \boldsymbol{\lambda}) \in T_{n}^{\prime} \times \Lambda_{n}$, the morphism $\mathbf{R} \mathbf{H}_{n \mid M_{n}^{\alpha}(\mathbf{t}, \boldsymbol{\lambda}, L)}$ is equal to $\mathbf{R H}_{\mathbf{t}, \boldsymbol{\lambda}}$ in (161).

Theorem 7.1. Under the notation and the assumption as above, we have the following assertions.

(1) For all $(\mathbf{t}, \boldsymbol{\lambda}) \in T_{n}^{\prime} \times \Lambda_{n}$, the Riemann-Hilbert correspondence $\mathbf{R H}_{\mathbf{t}, \boldsymbol{\lambda}}$ : $M_{n}^{\alpha}(\mathbf{t}, \boldsymbol{\lambda}, L) \longrightarrow \mathcal{R}\left(\mathcal{P}_{n, \mathbf{t}}\right)_{\mathbf{a}}$ in (161) is a bimeromorphic proper surjective morphism.

(2) For any $(\mathbf{t}, \boldsymbol{\lambda})$, let $\mathcal{R}\left(\mathcal{P}_{n, \mathbf{t}}\right)_{\mathbf{a}}^{\sharp}$ be the Zariski open subset of $\mathcal{R}\left(\mathcal{P}_{n, \mathbf{t}}\right)_{\mathbf{a}}$ whose closed points satisfy the conditions (141) and (142) in $\S 6$, and set $M_{n}^{\boldsymbol{\alpha}}(\mathbf{t}, \boldsymbol{\lambda}, L)^{\sharp}=\mathbf{R H}_{\mathbf{t}, \boldsymbol{\lambda}}^{-1}\left(\mathcal{R}\left(\mathcal{P}_{n, \mathbf{t}}\right)_{\mathbf{a}}^{\sharp}\right)$. Then the Riemann-Hilbert correspondence gives an analytic isomorphism

$$
\mathbf{R H}_{\mathbf{t}, \boldsymbol{\lambda}, \mid M_{n}^{\alpha}(\mathbf{t}, \boldsymbol{\lambda}, L)^{\sharp}}: M_{n}^{\boldsymbol{\alpha}}(\mathbf{t}, \boldsymbol{\lambda}, L)^{\sharp} \stackrel{\simeq}{\longrightarrow} \mathcal{R}\left(\mathcal{P}_{n, \mathbf{t}}\right)_{\mathbf{a}}^{\sharp} .
$$

(Note that if $\boldsymbol{\lambda}$ is generic (cf. Definition 2.3, (36), (37)), $\mathcal{R}\left(\mathcal{P}_{n, \mathbf{t}}\right)_{\mathbf{a}}^{\sharp}=$ $\mathcal{R}\left(\mathcal{P}_{n, \mathbf{t}}\right)_{\mathbf{a}}$, hence $\mathbf{R H}_{\mathbf{t}, \boldsymbol{\lambda}}$ gives an analytic isomorphism between $M_{n}^{\boldsymbol{\alpha}}(\mathbf{t}, \boldsymbol{\lambda}, L)$ and $\left.\mathcal{R}\left(\mathcal{P}_{n, \mathbf{t}}\right)_{\mathbf{a}} \cdot\right)$ 
(3) Let us set $\mathcal{R}\left(\mathcal{P}_{n, \mathbf{t}}\right)_{\mathbf{a}}^{\text {sing }}=\mathcal{R}\left(\mathcal{P}_{n, \mathbf{t}}\right)_{\mathbf{a}} \backslash \mathcal{R}\left(\mathcal{P}_{n, \mathbf{t}}\right)_{\mathbf{a}}^{\sharp}$. Then the codimension of $\mathcal{R}\left(\mathcal{P}_{n, \mathbf{t}}\right)_{\mathbf{a}}^{\text {sing }}$ in $\mathcal{R}\left(\mathcal{P}_{n, \mathbf{t}}\right)_{\mathbf{a}}$ is at least 2 .

(4) The symplectic structures $\Omega$ on $M_{n}^{\boldsymbol{\alpha}}(\mathbf{t}, \boldsymbol{\lambda}, L)$ and $\Omega_{1}$ on $\mathcal{R}\left(\mathcal{P}_{n, \mathbf{t}}\right)_{\mathbf{a}}^{\sharp}$ can be identified with each other via $\mathbf{R H}_{\mathbf{t}, \boldsymbol{\lambda}}$, that is,

$$
\Omega=\left(\mathbf{R H}_{\mathbf{t}, \boldsymbol{\lambda}, \mid \mathcal{R}\left(\mathcal{P}_{n, \mathbf{t}}\right)_{\mathbf{a}}^{\sharp}}\right)^{*}\left(\Omega_{1}\right) \quad \text { on } \quad M_{n}^{\boldsymbol{\alpha}}(\mathbf{t}, \boldsymbol{\lambda}, L)^{\sharp} \text {. }
$$

\section{Remark 7.1.}

(1) The moduli spaces $M_{n}^{\boldsymbol{\alpha}}(\mathbf{t}, \boldsymbol{\lambda}, L)$ and $\mathcal{R}\left(\mathcal{P}_{n, \mathbf{t}}\right)_{\mathbf{a}}$ are irreducible. (See $\S 8$ and $\S 9)$.

(2) The statement (165) is originally shown by Iwasaki in [Iw1], [Iw2].

Let us denote $\mathbf{R H}_{\mathbf{t}, \boldsymbol{\lambda}}$ in (161) simply by $\mathbf{R H}$. We first show the following

Lemma 7.1. Assume that $n \geq 4$ and that $\alpha_{i}(i=1, \ldots, n)$ are so general that all the semistable parabolic connections are stable. Then the morphism $\mathbf{R H}: M_{n}^{\alpha}(\mathbf{t}, \boldsymbol{\lambda}, L) \longrightarrow \mathcal{R}\left(\mathcal{P}_{n, \mathbf{t}}\right)_{\mathbf{a}}$ is a bimeromorphic surjective morphism.

Proof. Let $\mathcal{R}^{\mathrm{irr}}\left(\mathcal{P}_{n, \mathbf{t}}\right)_{\mathbf{a}}$ be the open subscheme of $\mathcal{R}\left(\mathcal{P}_{n, \mathbf{t}}\right)_{\mathbf{a}}$ whose points correspond to the irreducible representations. First we will show that $\mathcal{R}^{\operatorname{irr}}\left(\mathcal{P}_{n, \mathbf{t}}\right)_{\mathbf{a}}$ is contained in the image of $\mathbf{R H}$.

Let $M_{n}^{\mathrm{irr}}(\mathbf{t}, \boldsymbol{\lambda}, L)$ be the open subscheme of $M_{n}^{\boldsymbol{\alpha}}(\mathbf{t}, \boldsymbol{\lambda}, L)$ consisting of the points corresponding to the irreducible connections. Note that if $\left(E, \nabla_{E}, \varphi,\left\{l_{i}\right\}\right)$ is a parabolic connection such that $\left(E, \nabla_{E}\right)$ is an irreducible connection, we have $\left(E, \nabla_{E}, \varphi,\left\{l_{i}\right\}\right) \in M_{n}^{\boldsymbol{\alpha}}(\mathbf{t}, \boldsymbol{\lambda}, L)$. We consider the isomorphism of the moduli spaces

$$
\begin{gathered}
\operatorname{Elm}_{t_{i}}^{-}: M_{n}^{\mathrm{irr}}(\mathbf{t}, \boldsymbol{\lambda}, L) \stackrel{\sim}{\longrightarrow} M_{n}^{\mathrm{irr}}\left(\mathbf{t}, \boldsymbol{\lambda}^{\prime}, L\left(-t_{i}\right)\right) ; \\
\left(E, \nabla_{E}, \varphi,\left\{l_{i}\right\}\right) \mapsto\left(E^{\prime}, \nabla_{E^{\prime}}, \varphi^{\prime},\left\{l_{i}^{\prime}\right\}\right),
\end{gathered}
$$

where $E^{\prime}=\operatorname{ker}\left(E \rightarrow E_{t_{i}} / l_{i}\right), \nabla_{E^{\prime}}$ is a connection on $E^{\prime}$ induced by $\nabla_{E}$, $l_{i}^{\prime}=\operatorname{ker}\left(E_{t_{i}}^{\prime} \rightarrow E_{t_{i}}\right), l_{j}^{\prime}=l_{j}$ for $j \neq i, \lambda_{i}^{\prime}=1+\operatorname{res}_{t_{i}}\left(\nabla_{L}\right)-\lambda_{i}, \lambda_{j}^{\prime}=\lambda_{j}$ for $j \neq i$ and $\varphi^{\prime}: \bigwedge^{2} E^{\prime} \stackrel{\sim}{\rightarrow} L\left(-t_{i}\right)$ is the horizontal isomorphism induced by $\varphi$. We also consider the isomorphisms of the moduli spaces

$$
\begin{gathered}
\otimes \mathcal{O}\left(t_{i}\right): M_{n}^{\mathrm{irr}}(\mathbf{t}, \boldsymbol{\lambda}, L) \longrightarrow M_{n}^{\mathrm{irr}}\left(\mathbf{t}, \boldsymbol{\lambda}^{\prime}, L \otimes \mathcal{O}\left(2 t_{i}\right)\right) ; \\
\left(E, \nabla_{E}, \varphi,\left\{l_{i}\right\}\right) \mapsto\left(E \otimes \mathcal{O}\left(t_{i}\right), \nabla_{E \otimes \mathcal{O}\left(t_{i}\right)}, \varphi \otimes 1,\left\{\left.l_{i}^{\prime} \otimes \mathcal{O}\left(t_{i}\right)\right|_{t_{i}}\right\}\right),
\end{gathered}
$$


where $\lambda_{i}^{\prime}=\lambda_{i}-1$ and $\lambda_{j}^{\prime}=\lambda_{j}$ for $j \neq i$ and we consider for $\lambda_{i} \neq \operatorname{res}_{t_{i}}\left(\nabla_{L}\right)-\lambda_{i}$, an isomorphism

$$
s_{i}: M_{n}^{\mathrm{irr}}(\mathbf{t}, \boldsymbol{\lambda}, L) \stackrel{\sim}{\longrightarrow} M_{n}^{\mathrm{irr}}\left(\mathbf{t}, \boldsymbol{\lambda}^{\prime}, L\right) ; \quad\left(E, \nabla_{E}, \varphi,\left\{l_{i}\right\}\right) \mapsto\left(E, \nabla_{E}, \varphi,\left\{l_{i}^{\prime}\right\}\right),
$$

where $\lambda_{i}^{\prime}=\operatorname{res}_{t_{i}}\left(\nabla_{L}\right)-\lambda_{i}, \lambda_{j}^{\prime}=\lambda_{j}$ for $j \neq i, l_{i}^{\prime}=\operatorname{ker}\left(\operatorname{res}_{t_{i}}\left(\nabla_{E}\right)-\lambda_{i}^{\prime}\right)$ and $l_{j}^{\prime}=l_{j}$ for $j \neq i$. Note that these isomorphisms all commute with the Riemann-Hilbert morphism RH.

Now we fix $\left(\lambda_{1}, \ldots, \lambda_{n}\right) \in \mathbf{C}^{n}$ and put $\lambda_{i}^{+}:=\lambda_{i}, \lambda_{i}^{-}:=\operatorname{res}_{t_{i}}\left(\nabla_{L}\right)-\lambda_{i}$ for $i=1, \ldots, n$. Applying a certain composition of $\operatorname{Elm}_{t_{i}}^{-}, \otimes \mathcal{O}_{\mathbf{P}^{1}}\left(t_{i}\right)$ and $s_{i}$ for $i=1, \ldots, n$, we obtain an isomorphism

$$
\tau: M_{n}^{\mathrm{irr}}(\mathbf{t}, \boldsymbol{\lambda}, L) \stackrel{\sim}{\longrightarrow} M_{n}^{\mathrm{irr}}\left(\mathbf{t}, \boldsymbol{\lambda}^{\prime}, L^{\prime}\right),
$$

where $\lambda_{i}^{\prime}:=\lambda_{i}+m_{i}^{+}$and $\operatorname{res}_{t_{i}}\left(\nabla_{L^{\prime}}\right)=\operatorname{res}_{t_{i}}\left(\nabla_{L}\right)+m_{i}^{+}+m_{i}^{-}$for some integers $m_{i}^{+}, m_{i}^{-} \in \mathbf{Z}$ such that $0 \leq \operatorname{Re}\left(\lambda_{i}^{+}+m_{i}^{+}\right)<1,0 \leq \operatorname{Re}\left(\lambda_{i}^{-}+m_{i}^{-}\right)<1$ for $1 \leq i \leq n$.

Let $N_{n}^{\text {irr }}\left(\mathbf{t}, \boldsymbol{\lambda}^{\prime}, L^{\prime}\right)$ be the moduli space of rank 2 irreducible connections $\left(E, \nabla_{E}\right)$ with a horizontal isomorphism $\bigwedge^{2} E \stackrel{\sim}{\rightarrow} L^{\prime} \operatorname{such}$ that $\operatorname{det}\left(\operatorname{res}_{t_{i}}\left(\nabla_{E}\right)-\right.$ $\left.\lambda_{i}^{\prime}\right)=0$ for $i=1, \ldots, n$. By [[Del70], Proposition 5.4], we obtain an isomorphism

$$
\operatorname{rh}: N_{n}^{\mathrm{irr}}\left(\mathbf{t}, \boldsymbol{\lambda}^{\prime}, L^{\prime}\right) \stackrel{\sim}{\longrightarrow} \mathcal{R}^{\mathrm{irr}}\left(\mathcal{P}_{n, \mathbf{t}}\right)_{\mathbf{a}}
$$

There is a canonical surjective morphism

$$
M_{n}^{\mathrm{irr}}\left(\mathbf{t}, \boldsymbol{\lambda}^{\prime}, L^{\prime}\right) \rightarrow N_{n}^{\mathrm{irr}}\left(\mathbf{t}, \boldsymbol{\lambda}^{\prime}, L^{\prime}\right)
$$

which is obtained by forgetting parabolic structures. Composing $\tau$, (167) and rh, we obtain a surjective morphism

$$
\mathbf{R H}: M_{n}^{\mathrm{irr}}(\mathbf{t}, \boldsymbol{\lambda}, L) \longrightarrow \mathcal{R}^{\mathrm{irr}}\left(\mathcal{P}_{n, \mathbf{t}}\right)_{\mathbf{a}} .
$$

Note that the morphism (167) is isomorphic except on the locus where the parabolic structures are not uniquely determined by $\left(E, \nabla_{E}\right)$, namely,

$$
\begin{aligned}
& M_{n}^{\text {app }}\left(\mathbf{t}, \boldsymbol{\lambda}^{\prime}, L^{\prime}\right) \\
& \quad=\left\{\left(E, \nabla_{E}, \varphi,\left\{l_{j}\right\}\right) \in M_{n}^{i r r}\left(\mathbf{t}, \boldsymbol{\lambda}^{\prime}, L^{\prime}\right) \mid \operatorname{Res}_{t_{i}}\left(\nabla_{E}\right)=O \text { or } \frac{1}{2} \operatorname{id}_{E_{t_{i}}} \text { for some } i\right\}
\end{aligned}
$$

whose image in $\mathcal{R}\left(\mathcal{P}_{n, \mathbf{t}}\right)_{\mathbf{a}}$ is

$$
\mathcal{R}^{\text {app }}\left(\mathcal{P}_{n, \mathbf{t}}\right)_{\mathbf{a}}=\left\{\rho \in \mathcal{R}^{i r r}\left(\mathcal{P}_{n, \mathbf{t}}\right)_{\mathbf{a}} \mid \rho\left(\gamma_{i}\right)= \pm \text { id for some } i\right\} .
$$


Thus the restriction of $\mathbf{R H}$

$$
\begin{aligned}
& M_{n}^{\boldsymbol{\alpha}}(\mathbf{t}, \boldsymbol{\lambda}, L)^{\sharp} \\
& =M_{n}^{\mathrm{irr}}(\mathbf{t}, \boldsymbol{\lambda}, L) \backslash \tau^{-1}\left(M_{n}^{\mathrm{app}}\left(\mathbf{t}, \boldsymbol{\lambda}^{\prime}, L^{\prime}\right)\right) \stackrel{\mathrm{RH}}{\longrightarrow} \mathcal{R}^{\mathrm{irr}}\left(\mathcal{P}_{n, \mathbf{t}}\right)_{\mathbf{a}} \backslash \mathcal{R}^{\mathrm{app}}\left(\mathcal{P}_{n, \mathbf{t}}\right)_{\mathbf{a}}=\mathcal{R}\left(\mathcal{P}_{n, \mathbf{t}}\right)_{\mathbf{a}}^{\sharp}
\end{aligned}
$$

is an isomorphism. Since $\operatorname{dim} \mathcal{R}^{\operatorname{app}}\left(\mathcal{P}_{n, \mathbf{t}}\right)_{\mathbf{a}}<\operatorname{dim} \mathcal{R}^{\operatorname{irr}}\left(\mathcal{P}_{n, \mathbf{t}}\right)_{\mathbf{a}}$ for $n \geq 4$, $\mathbf{R H}$ is a bimeromorphic morphism.

Next we will show that $M_{n}^{\text {red }}(\mathbf{t}, \boldsymbol{\lambda}, L) \rightarrow \mathcal{R}^{\text {red }}\left(\mathcal{P}_{n, \mathbf{t}}\right)_{\mathbf{a}}$ is surjective. Take any point $[\rho] \in \mathcal{R}^{\mathrm{red}}\left(\mathcal{P}_{n, \mathbf{t}}\right)_{\mathbf{a}}$. Then the representation $\rho$ is Jordan equivalent to the representation $\rho_{1} \oplus \rho_{2}$ for some one dimensional representations $\rho_{1}, \rho_{2}$ of $\pi_{1}\left(\mathbf{P}^{1} \backslash D(\mathbf{t}), *\right)$. Put

$$
U_{n, \mathbf{a}}:=\left\{\begin{array}{l|l}
\left(M_{1}, \ldots, M_{n-1}\right) \in S L_{2}(\mathbf{C})^{n-1} & \begin{array}{l}
\operatorname{Tr}\left(M_{i}\right)=a_{i}(1 \leq i \leq n-1), \\
\operatorname{Tr}\left(\left(M_{1} M_{2} \cdots M_{n-1}\right)^{-1}\right)=a_{n}
\end{array}
\end{array}\right\} .
$$

Then $U_{n, \mathbf{a}}$ is irreducible by Proposition 8.2. Let $\Phi_{n}: U_{n, \mathbf{a}} \rightarrow \mathcal{R}\left(\mathcal{P}_{n, \mathbf{t}}\right)_{\mathbf{a}}$ be the quotient map. Then there exists a point $p_{0} \in U_{n, \mathbf{a}}$ such that $\Phi_{n}\left(p_{0}\right)=$ $[\rho]$. Since $U_{n, \mathbf{a}}$ is irreducible, there exists a smooth irreducible curve $C$, a point $p$ of $C$ and a morphism $f: C \rightarrow U_{n, \mathbf{a}}$ such that $f(p)=p_{0}$ and that $\Phi_{n}(f(C)) \cap \mathcal{R}^{\operatorname{irr}}\left(\mathcal{P}_{n, \mathbf{t}}\right)_{\mathbf{a}} \neq \emptyset$. From [[Del70], Proposition 5.4], there exists an analytic flat family $\left(\tilde{E}, \nabla_{\tilde{E}}, \tilde{\varphi}\right)$ of connections such that $\left.\operatorname{ker} \nabla_{\tilde{E}}\right|_{\left(\mathbf{P}^{1} \backslash D(\mathbf{t})\right) \times C}$ is equivalent to the flat family of local systems on $\left(\mathbf{P}^{1} \backslash D(\mathbf{t})\right) \times C$ over $C$ induced by the morphism $f$. Applying certain elementary transformations and tensoring line bundles to $\left(\tilde{E}, \nabla_{\tilde{E}}, \tilde{\varphi}\right)$, we may assume that the eigenvalues of $\operatorname{res}_{t_{i}}(\tilde{E})$ are $\lambda_{i}$ and $\operatorname{res}_{t_{i}}\left(\nabla_{L}\right)-\lambda_{i}$ for $i=1, \ldots, n$. We can construct a flat family of parabolic structures $\left\{\tilde{l}_{i}\right\}$ and $\left(\tilde{E}, \nabla_{\tilde{E}}, \tilde{\varphi},\left\{\tilde{l}_{i}\right\}\right)$ becomes a flat family of parabolic connections. Taking the completion at $p$, we obtain a flat family of parabolic connections $\left(E, \nabla_{E}, \varphi,\left\{l_{i}\right\}\right)$ on $\mathbf{P}_{\mathbf{C}[[x]]}^{1}$ over $\mathbf{C}[[x]]$. By Corollary 5.1, there exists a flat family $\left(E^{\prime}, \nabla_{E^{\prime}}, \varphi^{\prime},\left\{l^{\prime}\right\}\right)$ of $\boldsymbol{\alpha}$-semistable parabolic connections such that $\left(E, \nabla_{E}, \varphi,\left\{l_{i}\right\}\right) \otimes \mathbf{C}((x)) \cong\left(E^{\prime}, \nabla_{E^{\prime}}, \varphi^{\prime},\left\{l^{\prime}\right\}\right) \otimes \mathbf{C}((x))$ and $g r\left(\left(E^{\prime}, \nabla_{E^{\prime}}\right) \otimes\right.$ $\mathbf{C}[[x]] /(x)) \cong \operatorname{gr}\left(\left(E, \nabla_{E}\right) \otimes \mathbf{C}[[x]] /(x)\right)$. Then $\left(E^{\prime}, \nabla_{E^{\prime}}, \varphi^{\prime},\left\{l^{\prime}\right\}\right)$ determines a morphism Spec $\mathbf{C}[[x]] \rightarrow M_{n}^{\boldsymbol{\alpha}}(\mathbf{t}, \boldsymbol{\lambda}, L)$. If $q$ is the image of the closed point by this morphism, then we have $\mathbf{R H}(q)=[\rho]$.

\subsubsection{Proof of Theorem 7.1 except for the properness of $\mathbf{R H}_{\mathbf{t}, \boldsymbol{\lambda}}$}

Proof. Here we prove the assertions in Theorem 7.1 except for the properness of $\mathbf{R H}_{\mathbf{t}, \boldsymbol{\lambda}}$ which will be proved in Proposition 10.1. The first assertion except for the properness follows from Lemma 7.1 and the second assertion is proved in the proof of Lemma 7.1. The last assertion follows from 
these assertions and Lemma 6.6. For the third assertion recall the definition of $\mathcal{R}^{i r r}\left(\mathcal{P}_{n, \mathbf{t}}\right)_{\mathbf{a}}$ and $\mathcal{R}^{a p p}\left(\mathcal{P}_{n, \mathbf{t}}\right)_{\mathbf{a}}$ in the proof of Lemma 7.1. Let us set $\mathcal{R}^{\text {red }}\left(\mathcal{P}_{n, \mathbf{t}}\right)_{\mathbf{a}}=\mathcal{R}\left(\mathcal{P}_{n, \mathbf{t}}\right)_{\mathbf{a}} \backslash \mathcal{R}^{i r r}\left(\mathcal{P}_{n, \mathbf{t}}\right)_{\mathbf{a}}$. Then we see that

$$
\mathcal{R}\left(\mathcal{P}_{n, \mathbf{t}}\right)_{\mathbf{a}}^{\text {sing }}=\mathcal{R}^{r e d}\left(\mathcal{P}_{n, \mathbf{t}}\right)_{\mathbf{a}} \cup \mathcal{R}^{a p p}\left(\mathcal{P}_{n, \mathbf{t}}\right)_{\mathbf{a}}
$$

If $[\rho] \in \mathcal{R}^{r e d}\left(\mathcal{P}_{n, \mathbf{t}}\right)_{\mathbf{a}}$, then $\rho$ is a reducible representation. Then the semisimplification of $\rho$ is a direct sum of one dimensional representation $\rho_{1}, \rho_{2}$. Since $\wedge^{2} \rho$ is trivial, $\rho_{2} \simeq \rho_{1}^{-1}$. Moreover since $\operatorname{Tr}\left[\rho\left(\gamma_{i}\right)\right]=a_{i}$ are fixed for all $1 \leq i \leq n$, we see that Jordan equivalence class of $\rho$, which is equal to the Jordan equivalence class of $\rho_{1} \oplus \rho_{1}^{-1}$, has finitely many possibility. Hence $\mathcal{R}^{r e d}\left(\mathcal{P}_{n, \mathbf{t}}\right)_{\mathbf{a}}$ is a zero dimensional subscheme. Moreover for a closed point $[\rho] \in \mathcal{R}^{a p p}\left(\mathcal{P}_{n, \mathbf{t}}\right)_{\mathbf{a}}, \rho$ is irreducible and $\rho\left(\gamma_{i}\right)= \pm i d$ for some $i$ by definition. This means that $\rho$ is determined by $\rho\left(\gamma_{j}\right)$ for $j \neq i$ and so $\operatorname{dim} \mathcal{R}^{a p p}\left(\mathcal{P}_{n, \mathbf{t}}\right)_{\mathbf{a}}=\operatorname{dim} \mathcal{R}\left(\mathcal{P}_{n-1, \mathbf{t}}\right)_{\mathbf{a}^{\prime}}$. Noting that $\operatorname{dim} \mathcal{R}\left(\mathcal{P}_{n, \mathbf{t}}\right)_{\mathbf{a}}=2 n-6$ for $n \geq 3$, we have $\operatorname{dim} \mathcal{R}^{a p p}\left(\mathcal{P}_{n, \mathbf{t}}\right)_{\mathbf{a}}=2 n-8$ for $n \geq 4$. In both cases, if $n \geq 4$, the codimensions of the subschemes are at least 2 .

\section{$\S 7.3 . \quad$ The case of $n=4$}

In the case of $n=4$, let us recall the isomorphism

$$
T_{4}^{\prime} / P G L_{2} \simeq B=\mathbf{P}^{1}-\{0,1, \infty\}
$$

where $B$ is one-dimensional space of time variables as usual. Here the group $P G L_{2}$ acts on the base space $\mathbf{P}^{1}$ by linear fractional transformations. Therefore the family and the morphism can be descended and one obtains the following commutative diagram:

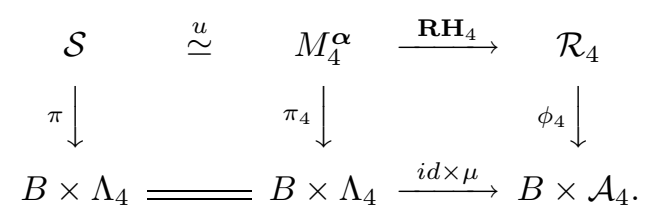

Here the family $\pi: \mathcal{S} \longrightarrow B \times \Lambda_{4}$ is the family of Okamoto space of initial conditions. The isomorphism $u$ will be constructed in [IIS2]. 


\section{$\S 8$. Irreducibility of $\mathcal{R}\left(\mathcal{P}_{n, \mathrm{t}}\right)_{\mathrm{a}}$}

As in Lemma 4.1, we have the natural quotient morphism

$$
\begin{aligned}
\Phi_{n}: S L_{2}(\mathbf{C})^{n-1} & \longrightarrow \mathcal{R}\left(\mathcal{P}_{n, \mathbf{t}}\right) \simeq \operatorname{Spec}\left[\left(R_{n-1}\right)^{A d\left(S L_{2}(\mathbf{C})\right)}\right] \\
\left(M_{1}, M_{2}, \ldots, M_{n-1}\right) & \mapsto\left[M_{1}, M_{2}, \ldots, M_{n-1}\right]
\end{aligned}
$$

where $R_{n-1}$ denotes the affine coordinate ring of $S L_{2}(\mathbf{C})^{n-1}$. Under this quotient morphism, for $\mathbf{a}=\left(a_{1}, \ldots, a_{n}\right) \in \mathcal{A}_{n}=\mathbf{C}^{n}$, the subscheme $\mathcal{R}\left(\mathcal{P}_{n, \mathbf{t}}\right)_{\mathbf{a}}$ in (97) is isomorphic to

$$
\mathcal{R}\left(\mathcal{P}_{n, \mathbf{t}}\right)_{\mathbf{a}}=\left\{\begin{array}{l|l}
{\left[M_{1}, \ldots, M_{n-1}\right] \in \mathcal{R}\left(\mathcal{P}_{n, \mathbf{t}}\right)} & \begin{array}{l}
\operatorname{Tr}\left(M_{i}\right)=a_{i},(1 \leq i \leq n-1), \\
\operatorname{Tr}\left(M_{1} M_{2} \cdots M_{n-1}\right)^{-1}=a_{n}
\end{array}
\end{array}\right\} .
$$

Proposition 8.1. Assume that $n \geq 4$. The affine scheme $\mathcal{R}\left(\mathcal{P}_{n, \mathbf{t}}\right)_{\mathbf{a}}$ is irreducible.

Set $U_{n, \mathbf{a}}:=\Phi_{n}^{-1}\left(\mathcal{R}\left(\mathcal{P}_{n, \mathbf{t}}\right)_{\mathbf{a}}\right)$ so that we have a surjective morphism $U_{n, \mathbf{a}} \longrightarrow$ $\mathcal{R}\left(\mathcal{P}_{n, \mathbf{t}}\right)_{\mathbf{a}}$ of schemes. Because $\operatorname{Tr}\left(M_{1} M_{2} \cdots M_{n-1}\right)^{-1}=\operatorname{Tr}\left(M_{1} M_{2} \cdots M_{n-1}\right)$ for $M_{i} \in S L_{2}(\mathbf{C})$, we have

$$
U_{n, \mathbf{a}}=\left\{\begin{array}{l|l}
\left(M_{1}, \ldots, M_{n-1}\right) \in S L_{2}(\mathbf{C})^{n-1} & \begin{array}{l}
\operatorname{Tr}\left(M_{i}\right)=a_{i}, 1 \leq i \leq n-1, \\
\operatorname{Tr}\left(M_{1} M_{2} \cdots M_{n-1}\right)=a_{n}
\end{array}
\end{array}\right\} .
$$

Then it suffices to show the following

Proposition 8.2. The scheme $U_{n, \mathbf{a}}$ is irreducible.

Let us prove some easy lemma which we will use later. The proof of the following lemma is easy and we omit it.

Lemma 8.1. Fix $a \in \mathbf{C}$ and define

$$
V_{a}=\left\{A=\left(\begin{array}{cc}
s & t \\
u & v
\end{array}\right) \in S L_{2}(\mathbf{C}) \mid \operatorname{Tr}(A)=a\right\} .
$$

(1) Then $V_{a}$ is an irreducible affine subscheme of $\mathbf{C}^{3}$.

(2) Let us define a quadratic hypersurface in $\mathbf{P}_{\mathbf{C}}^{3}$ as:

$$
\bar{V}_{a}:=\left\{[x: y: z: w] \in \mathbf{P}_{\mathbf{C}}^{3} \mid x^{2}-a x w+w^{2}+y z=0\right\} .
$$


Then we have an isomorphism $V_{a} \simeq \bar{V}_{a} \backslash\{w=0\}$, that is, $\bar{V}_{a}$ is a compactification of $V_{a}$. If $a \neq \pm 2, \bar{V}_{a}$ is a smooth quadric hypersurface, and if $a= \pm 2, \bar{V}_{a}$ is a cone over a conic and have a unique singular point at $p_{a}=[x: y: z: w]=[a / 2: 0: 0: 1]$.

Fix $\mathbf{a}=\left(a_{1}, \ldots, a_{n}\right) \in \mathcal{A}_{n}$ and set $\mathbf{a}^{\prime}=\left(a_{1}, \ldots, a_{n-1}\right)$. Using the notation in Lemma 8.1 , we set

$$
V_{\mathbf{a}^{\prime}}:=V_{a_{1}} \times V_{a_{2}} \times \cdots \times V_{a_{n-1}} \subset \bar{V}_{\mathbf{a}^{\prime}}:=\bar{V}_{a_{1}} \times \bar{V}_{a_{2}} \cdots \times \bar{V}_{a_{n-1}}
$$

It is obvious that $U_{n, \mathbf{a}}$ is a Cartier divisor of the scheme

$$
V_{\mathbf{a}^{\prime}}=\left\{\left(M_{1}, \ldots, M_{n-1}\right) \in S L_{2}(\mathbf{C}) \mid \operatorname{Tr}\left(M_{i}\right)=a_{i}, 1 \leq i \leq n-1\right\}
$$

defined by the equation

$$
\operatorname{Tr}\left(M_{1} M_{2} \cdots M_{n-1}\right)=a_{n}
$$

Again from Lemma 8.1, we can introduce a homogeneous coordinates $\left[x_{i}: y_{i}\right.$ : $\left.z_{i}: w_{i}\right] \in \mathbf{P}_{\mathbf{C}}^{3}$ such that

$$
\bar{V}_{a_{i}}=\left\{\left[x_{i}: y_{i}: z_{i}: w_{i}\right] \in \mathbf{P}_{\mathbf{C}}^{3} \mid F_{a_{i}}=x_{i}^{2}-a_{i} x_{i} w_{i}+w_{i}^{2}+y_{i} z_{i}=0\right\}
$$

Let us denote by $\bar{U}_{n, \mathbf{a}}$ the closure of $U_{n, \mathbf{a}} \subset V_{\mathbf{a}^{\prime}}$ in $\bar{V}_{\mathbf{a}^{\prime}} \subset\left(\mathbf{P}_{\mathbf{C}}^{3}\right)^{n-1}$. It is easy to see that $\bar{U}_{n, \mathbf{a}}$ is also a Cartier divisor in $\bar{V}_{\mathbf{a}^{\prime}}$.

For $1 \leq i \leq n-1$, set $T_{n-2, i}=V_{a_{1}} \times \cdots \times \widehat{V}_{a_{i}} \times \cdots \times V_{a_{n-1}}$ and $\bar{T}_{n-2, i}=$ $\bar{V}_{a_{2}} \times \cdots \times{\widehat{\bar{V}_{a_{i}}}} \times \cdots \times \bar{V}_{a_{n-1}}$ (omitting $i$-th factors) and consider the $i$-th projections

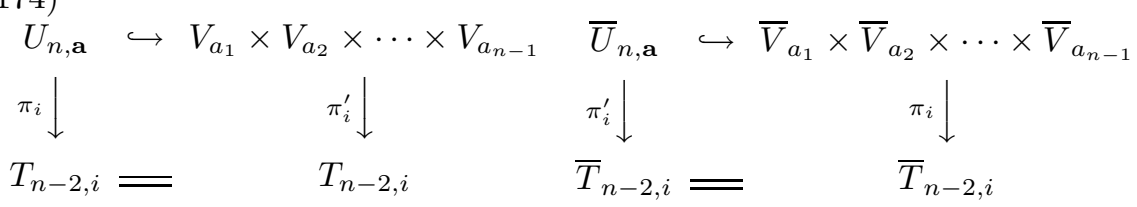

Lemma 8.2. For each $1 \leq i \leq n-1$, the family $\pi_{i}: \bar{U}_{n, \mathbf{a}} \longrightarrow \bar{T}_{n-2, i}$ can be considered as a family of hyperplane sections of $\bar{V}_{a_{i}} \subset \mathbf{P}_{\mathbf{C}}^{3}$ parametrized by $\bar{T}_{n-2, i}$. Therefore $\bar{U}_{n, \mathbf{a}} \subset \bar{V}_{\mathbf{a}^{\prime}}$ is a hypersurface defined by a multihomogeneous polynomial

$$
H_{\mathbf{a}}=H_{\mathbf{a}}\left(x_{1}, y_{1}, z_{1}, w_{1}, \ldots, x_{n-1}, y_{n-1}, z_{n-1}, w_{n-1}\right)
$$

in the homogeneous coordinate ring of $\left(\mathbf{P}_{\mathbf{C}}^{3}\right)^{n-1}$ of multi-degree $(1, \ldots, 1)$. 
Proof. First we prove the assertion for $i=1$. For simplicity, we set $T=T_{n-2,1}$ and $\bar{T}=\bar{T}_{n-2,1}$ and we write as $\pi_{1}: U_{n, \mathbf{a}} \longrightarrow T, \bar{\pi}_{1}: \bar{U}_{n, \mathbf{a}} \longrightarrow \bar{T}$. Take an element $\left(M_{2}, M_{3}, \ldots, M_{n-1}\right) \in T$ and set

$$
M_{2} M_{3} \cdots M_{n-1}=\left(\begin{array}{ll}
f_{1} & f_{2} \\
f_{3} & f_{4}
\end{array}\right)
$$

and $M_{1}=\left(\begin{array}{cc}s & t \\ u & a_{1}-s\end{array}\right) \in V_{a_{1}}$ with $s\left(a_{1}-s\right)-t u=1$. Then we can write as

$$
\begin{aligned}
M_{1}\left(M_{2} \cdots M_{n-1}\right) & =\left(\begin{array}{cc}
s & t \\
u & a_{1}-s
\end{array}\right)\left(\begin{array}{ll}
f_{1} & f_{2} \\
f_{3} & f_{4}
\end{array}\right) \\
& =\left(\begin{array}{cc}
s f_{1}+t f_{3} & s f_{2}+t f_{4} \\
f_{3}\left(a_{1}-s\right)+u f_{1} & \left(a_{1}-s\right) f_{4}+u f_{2}
\end{array}\right) .
\end{aligned}
$$

Hence the Cartier divisor $U_{n, \mathbf{a}} \subset V_{\mathbf{a}^{\prime}}$ is defined by the polynomial

$$
\begin{aligned}
\operatorname{Tr}\left(M_{1} M_{2} \cdots M_{n-1}\right)-a_{n} & =s f_{1}+t f_{3}+\left(a_{1}-s\right) f_{4}+u f_{2}-a_{n} \\
& =\left(f_{1}-f_{4}\right) s+f_{3} t+f_{2} u+\left(a_{1} f_{4}-a_{n}\right) .
\end{aligned}
$$

First, we will show that any irreducible component of $U_{n, \mathbf{a}}$ is not a pullback divisor via $\pi_{1}$. Consider the subscheme $Z$ of $T$ defined by the ideal generated by the following elements:

$$
f_{1}-f_{4}, f_{3}, f_{2}, a_{1} f_{4}-a_{n}
$$

Then, it suffices to show that the codimension of $Z$ in $T$ is at least 2. Recall that $T$ is a product of $V_{a_{i}}$ 's for $i=2, \ldots, n-1$.

If $n \geq 4$, let us consider the natural projection $\varphi: Z \longrightarrow V_{a_{2}} \times \cdots \times V_{a_{n-2}}$. We will show that every closed fiber of $\varphi$ consists of a finite number of points or becomes empty, which means that codimension of $Z$ in $T$ is at least 2 . For this purpose, let us set

$$
M_{2} \cdots M_{n-2}=\left(\begin{array}{cc}
g_{1} & g_{2} \\
g_{3} & g_{4}
\end{array}\right)
$$

and

$$
M_{n-1}=\left(\begin{array}{cc}
s & t \\
u & b-s
\end{array}\right) .
$$

(Note that $g_{1} g_{4}-g_{2} g_{3}=1, s(b-s)-t u=1, s=s_{n-1}, t=t_{n-1}, u=t_{n-1}, b=$ $\left.a_{n-1}\right)$. Since

$$
\left(\begin{array}{ll}
f_{1} & f_{2} \\
f_{3} & f_{4}
\end{array}\right)=M_{2} \cdots M_{n-2} M_{n-1}=\left(\begin{array}{ll}
g_{1} & g_{2} \\
g_{3} & g_{4}
\end{array}\right)\left(\begin{array}{cc}
s & t \\
u & b-s
\end{array}\right)
$$




$$
=\left(\begin{array}{ll}
g_{1} s+g_{2} u & g_{1} t+g_{2}(b-s) \\
g_{3} s+g_{4} u & g_{3} t+g_{4}(b-s)
\end{array}\right),
$$

the ideal of $Z$ contains the following element

$$
\begin{aligned}
f_{1}-f_{4} & =\left(g_{1}+g_{4}\right) s+g_{2} u-g_{3} t-g_{4} b \\
f_{2} & =g_{1} t+g_{2}(b-s) \\
f_{3} & =g_{3} s+g_{4} u .
\end{aligned}
$$

Using the relations $g_{1} g_{4}-g_{2} g_{3}=1, s(b-s)-t u=1$, from these elements we can obtain the following elements of the ideal of $Z$

$$
s^{2}-g_{4}^{2}, \quad t^{2}-g_{2}^{2}, \quad u^{2}-g_{3}^{2}
$$

This means that every closed fiber of the projection $\varphi: Z \longrightarrow V_{a_{2}} \times \cdots \times V_{a_{n-2}}$ consists of finitely many points or becomes the empty set as desired. Let us recall the natural projection $\pi_{1}: U_{n, \mathbf{a}} \longrightarrow T$. The assertion implies that the Cartier divisor $U_{n, \mathbf{a}}$ defined by the polynomial (176) has no irreducible component which is a pullback Cartier divisor by $\pi_{1}$. Then, from the expression in (176), we conclude that the polynomial (176) is of degree 1 with respect to $s, t, u$ and hence the fibers of the compactifications $\bar{\pi}_{1}: \bar{U}_{n, \mathbf{a}} \longrightarrow \bar{T}$ of morphism $\pi_{1}$ are hyperplane sections of the quadric hypersurface $\bar{V}_{a_{1}} \subset \mathbf{P}_{\mathbf{C}}^{3}$. This proves the assertion for $i=1$. Since

$$
\operatorname{Tr}\left(M_{i} M_{i+1} \cdots M_{n-1} M_{1} \cdots M_{i-1}\right)=\operatorname{Tr}\left(M_{1} M_{2} \cdots M_{n-1}\right)
$$

the same is true for the $i$-th factor. Now we can conclude that $\bar{U}_{n, \mathbf{a}}$ is defined by the multi-homogeneous polynomial of $H_{\mathbf{a}}$ of multi-degree $(1, \ldots, 1)$.

Now we prove

Lemma 8.3. For any $\mathbf{a}=\left(a_{1}, \ldots, a_{n}\right) \in \mathcal{A}_{n}$ and $i, 1 \leq i \leq n-1$, the general fiber of $\pi_{i}: \bar{U}_{n, \mathbf{a}} \longrightarrow \bar{T}_{n-2, i}$ is irreducible and reduced.

Proof. By (177), we only have to prove the assertion for $i=1$. From now on, we set $T_{n-2}:=T_{n-2,1}, \bar{T}_{n-2}=\bar{T}_{n-2,1}$ and $\pi=\pi_{1}$, etc.

For $\left(M_{1}, M_{2}, M_{3}, \ldots, M_{n-1}\right) \in U_{n, \mathbf{a}}$, write

$$
M_{1}=\left(\begin{array}{cc}
s_{1} & t_{1} \\
u_{1} & a_{1}-s_{1}
\end{array}\right), \quad M_{2} M_{3} \cdots M_{n-1}=\left(\begin{array}{cc}
f_{1} & f_{2} \\
f_{3} & f_{4}
\end{array}\right) .
$$


Then for a fixed $\left(M_{2}, M_{3}, \ldots, M_{n-1}\right) \in T_{n-2}$, the fiber of $\pi_{\mid U_{n, \mathbf{a}}}: U_{n, \mathbf{a}} \longrightarrow T_{n-2}$ is defined by the equations

$$
s_{1} f_{1}+t_{1} f_{3}+\left(a_{1}-s_{1}\right) f_{4}+u_{1} f_{2}-a_{n}=0, \quad s_{1}^{2}-a_{1} s_{1}+1+t_{1} u_{1}=0
$$

of $(s, t, u) \in \mathbf{C}^{3}$. Therefore again for a fixed $\left(M_{2}, M_{3}, \ldots, M_{n-1}\right) \in T_{n-2}$ the equations of the fiber $\bar{U}_{\mathbf{f}}$ of $\pi: \bar{U}_{n, \mathbf{a}} \longrightarrow \bar{T}_{n}$ is given by

$\left(f_{1}-f_{4}\right) x_{1}+f_{3} y_{1}+f_{2} z_{1}+\left(a_{1} f_{4}-a_{n}\right) w_{1}=0, \quad x_{1}^{2}-a_{1} x_{1} w_{1}+w_{1}^{2}+y_{1} z_{1}=0$.

Recall that $\left[x_{1}: y_{1}: z_{1}: w_{1}\right]$ is the homogeneous coordinate for $\mathbf{P}_{\mathbf{C}}^{3}$ and $\bar{V}_{a_{1}}=\left\{x_{1}^{2}-a_{1} x_{1} w_{1}+w_{1}^{2}-y_{1} z_{1}=0\right\} \subset \mathbf{P}_{\mathbf{C}}^{3}$ is a quadric hypersurface. Therefore the fiber $\bar{U}_{\mathbf{f}}$ is a complete intersection of the quadric and the hyperplane defined by the equations above. In order to see that the general fiber $\bar{U}_{\mathbf{f}}$ is irreducible, it suffices to show that for a special choice of $\left(M_{2}, \ldots, M_{n}\right)$ the fiber is irreducible. First we consider the Zariski open subset defined by $z_{1} \neq 0$. Setting $X=$ $\frac{x_{1}}{z_{1}}, Y=\frac{y_{1}}{z_{1}}, W=\frac{w_{1}}{z_{1}}$, the equation above can be reduced to

(179) $\left(f_{1}-f_{4}\right) X+f_{3} Y+f_{2}+\left(a_{1} f_{4}-a_{n}\right) W=0, \quad Y=-X^{2}+a_{1} X W-W^{2}$.

Therefore the above open subscheme is isomorphic to an affine quadratic curve in $\mathbf{C}^{2}$ defined by

$$
-f_{3} X^{2}+a_{1} f_{3} X W-f_{3} W^{2}+\left(f_{1}-f_{4}\right) X+\left(a_{1} f_{4}-a_{n}\right) W+f_{2}=0 .
$$

It is easy to see that this affine quadric curve is reducible if and only if

$$
\operatorname{det}\left(\begin{array}{ccc}
-f_{3} & a_{1} f_{3} / 2 & \left(f_{1}-f_{4}\right) / 2 \\
a_{1} f_{3} / 2 & -f_{3} & \left(a_{1} f_{4}-a_{n}\right) / 2 \\
\left(f_{1}-f_{4}\right) / 2 & \left(a_{1} f_{4}-a_{n}\right) / 2 & f_{2}
\end{array}\right)=0 .
$$

Then in order to prove the Lemma, we only have to find $\left(M_{2}, M_{3}, \ldots, M_{n-1}\right) \in$ $T_{n-2}$ such that the equation (181) has no solution. For this purpose we choose the following matrices.

$$
M_{2}=\left(\begin{array}{cc}
0 & 1 \\
-1 & a_{2}
\end{array}\right), \quad M_{3} M_{4} \cdots M_{n-1}=\left(\begin{array}{cc}
c & p c^{-1} \\
0 & c^{-1}
\end{array}\right) .
$$

Here $c$ is a non-zero constant and $p$ is a free parameter. Then we have

$$
M_{2} M_{3} \cdots M_{n-1}=\left(\begin{array}{ll}
f_{1} & f_{2} \\
f_{3} & f_{4}
\end{array}\right)=\left(\begin{array}{cc}
0 & c^{-1} \\
-c & -p c^{-1}+a_{2} c^{-1}
\end{array}\right) .
$$


Then setting $f_{1}=0, f_{2}=c^{-1}, f_{3}=-c, f_{4}=\left(-p+a_{2}\right) c^{-1}$, the equation (181) becomes

$$
\operatorname{det}\left(\begin{array}{ccc}
c & -a_{1} c / 2 & \left(p-a_{2}\right) c^{-1} / 2 \\
-a_{1} c / 2 & c & \left(a_{1}\left(a_{2}-p\right) c^{-1}-a_{n}\right) / 2 \\
\left(p-a_{2}\right) c^{-1 / 2} & \left(a_{1}\left(a_{2}-p\right) c^{-1}-a_{n}\right) / 2 & c^{-1}
\end{array}\right)=0 .
$$

Expanding the determinant and multiplying a non-zero constant, we obtain the explicit equation

$$
p^{2}+\left(a_{1} a_{n-1} c-2 a_{2}\right) p+\left(a_{2}^{2}-a_{1} a_{2} a_{n} c-4 c^{2}+a_{1}^{2} c^{2}+a_{n}^{2} c^{2}\right)=0 .
$$

Since $a_{1}, a_{2}, a_{n-1}, c$ are fixed constant and $p$ is a free parameter, we can choose a value of $p$ so that the equation (183) has no solution. Therefore for such a choice, the affine quadratic curve (180) is irreducible and reduced, and then it is easy to conclude that the fiber $\bar{U}_{\mathbf{f}}$ defined by (178) is irreducible and reduced.

\section{Proof of Proposition 8.2}

Proof. In the proof of Lemma 8.2, we have shown that any irreducible component of $U_{n, \mathbf{a}}$ is not a pullback divisor via $\pi_{i}$ for $1 \leq i \leq n-1$. Since the Cartier divisor $\bar{U}_{n, \mathbf{a}}$ in $\bar{V}_{\mathbf{a}^{\prime}}=\bar{V}_{a_{1}} \times \cdots \times \bar{V}_{a_{n-1}}$ has the multi-degree $(1, \ldots, 1)$ with respect to the embeddings $\bar{V}_{a_{i}} \hookrightarrow \mathbf{P}_{\mathbf{C}}^{3}$, each closed fiber of $\bar{\pi}_{i}: \bar{U}_{n, \mathbf{a}} \longrightarrow \bar{T}_{n-2, i}$ is isomorphic to a hyperplane section of the quadric hypersurface $\bar{V}_{a_{i}}$. If $\bar{U}_{n, \mathbf{a}}$ is a reducible Cartier divisor in $\bar{V}_{\mathbf{a}^{\prime}}$, there exists an integer $i, 1 \leq i \leq n-1$ such that all closed fiber $\bar{\pi}_{i}$ is a reducible conic or a double line, and this contradicts to Lemma 8.3.

\section{$\S 9 . \quad$ Irreducibility of $M_{n}^{\alpha}(\mathbf{t}, \boldsymbol{\lambda}, L)$}

Proposition 9.1. $\quad M_{n}^{\alpha}(\mathbf{t}, \boldsymbol{\lambda}, L)$ is irreducible.

Proof. From the proof of Lemma 7.1,

$$
M_{n}^{\boldsymbol{\alpha}}(\mathbf{t}, \boldsymbol{\lambda}, L)^{\sharp} \stackrel{\mathbf{R H}}{\longrightarrow} \mathcal{R}\left(\mathcal{P}_{n, \mathbf{t}}\right)_{\mathbf{a}}^{\sharp}
$$

is an analytic isomorphism. Since $\mathcal{R}\left(\mathcal{P}_{n, \mathbf{t}}\right)_{\mathbf{a}}$ is irreducible by Proposition 8.1, $M_{n}^{\boldsymbol{\alpha}}(\mathbf{t}, \boldsymbol{\lambda}, L)^{\sharp}$ is also irreducible. If $M_{n}^{\boldsymbol{\alpha}}(\mathbf{t}, \boldsymbol{\lambda}, L)=M_{n}^{\boldsymbol{\alpha}}(\mathbf{t}, \boldsymbol{\lambda}, L)^{\sharp}$, there is nothing to prove. So assume that $2 \lambda_{i} \in \mathbf{Z}$ for some $i$ or $\sum_{i=1}^{n} \epsilon_{i} \lambda_{i} \in \mathbf{Z}$ for some 
$\left(\epsilon_{i}\right) \in\{ \pm 1\}^{n}$. Note that $M_{n}^{\boldsymbol{\alpha}}(\mathbf{t}, \boldsymbol{\lambda}, L)$ is smooth over $\mathbf{C}$ of equidimension $2 n-6$ (cf. Proposition 6.2). So it suffices to show that the dimension of $M_{n}^{\boldsymbol{\alpha}}(\mathbf{t}, \boldsymbol{\lambda}, L) \backslash$ $M_{n}^{\boldsymbol{\alpha}}(\mathbf{t}, \boldsymbol{\lambda}, L) \#$ is less than $2 n-6$.

First we consider the case $2 \lambda_{i} \in \mathbf{Z}$ for some $i$. By composing elementary transforms at $t_{i}$, we can obtain an isomorphism

$$
\tau: \mathcal{M}_{n}(\mathbf{t}, \boldsymbol{\lambda}, L) \stackrel{\sim}{\longrightarrow} \mathcal{M}_{n}\left(\mathbf{t}, \boldsymbol{\lambda}^{\prime}, L^{\prime}\right)
$$

of moduli stacks of parabolic connections without stability condition, where $\lambda_{i}^{\prime}=\operatorname{res}_{t_{i}}\left(\nabla_{L^{\prime}}\right) / 2$ and $\lambda_{j}^{\prime}=\lambda_{j}$ for $j \neq i$. Put

$A_{i}:=\left\{\begin{array}{l|l}\tau^{-1}\left(E, \nabla_{E}, \varphi,\left\{l_{j}\right\}\right) \in M_{n}^{\alpha}(\mathbf{t}, \boldsymbol{\lambda}, L) & \begin{array}{l}\left(E, \nabla_{E}\right) \text { is an irreducible } \\ \text { connection and } \operatorname{res}_{t_{i}}\left(\nabla_{E}\right)=\lambda_{i}^{\prime} \text { id }\end{array}\end{array}\right\}$.

Tensoring a certain line bundle with a connection having the residue $m / 2$ at $t_{i}$ for some integer $m$, we may assume that $\lambda_{i}^{\prime}=0$. Put

$$
\begin{aligned}
\tilde{\mathbf{t}} & :=\left(t_{1}, \ldots, t_{i-1}, t_{i+1}, \ldots, t_{n}\right), \\
\tilde{\boldsymbol{\lambda}} & :=\left(\lambda_{1}, \ldots, \lambda_{i-1}, \lambda_{i+1}, \ldots, \lambda_{n}\right) .
\end{aligned}
$$

Then $A_{i}$ consists of the irreducible $(\tilde{\mathbf{t}}, \tilde{\boldsymbol{\lambda}})$-parabolic connections $\left(E, \nabla_{E}, \varphi,\left\{l_{j}\right\}\right)$ with a one dimensional subspace $\left.l_{i} \subset \tau(E)\right|_{t_{i}}$. So we have $\operatorname{dim} A_{i}=2(n-1)-$ $6+1<2 n-6$.

Next consider the case $\sum_{i=1}^{n} \epsilon_{i} \lambda_{i} \in \mathbf{Z}$ with $\epsilon_{i} \in\{ \pm 1\}$. As in the proof of Lemma 7.1, we can obtain, by composing elementary transforms, an isomorphism

$$
\tau: \mathcal{M}_{n}(\mathbf{t}, \boldsymbol{\lambda}, L) \stackrel{\sim}{\longrightarrow} \mathcal{M}_{n}\left(\mathbf{t}, \boldsymbol{\lambda}^{\prime}, L^{\prime}\right)
$$

of moduli stacks of parabolic connections without stability condition, where $\operatorname{deg} L^{\prime}=0, \epsilon_{i} \lambda_{i}-\lambda_{i}^{\prime} \in \mathbf{Z}$ for any $i, \sum_{i=1}^{n} \lambda_{i}^{\prime}=0$ and $\lambda_{i}^{\prime}=\operatorname{res}_{t_{i}}\left(\nabla_{L^{\prime}}\right) / 2$ if $2 \lambda_{i} \in$ Z. Put

$$
B:=\left\{\begin{array}{l|l}
\tau^{-1}\left(E, \nabla_{E}, \varphi,\left\{l_{i}\right\}\right) \in M_{n}^{\alpha}(\mathbf{t}, \boldsymbol{\lambda}, L) & \begin{array}{l}
\text { there exists a subconnection } \\
\left(F, \nabla_{F}\right) \subset\left(E, \nabla_{E}\right) \text { satisfying } \\
\operatorname{res}_{t_{i}}\left(\nabla_{F}\right)=\lambda_{i}^{\prime} \text { for any } i .
\end{array}
\end{array}\right\}
$$

Take the sections $\omega_{1}, \omega_{3} \in H^{0}\left(\Omega_{\mathbf{P}^{1}}^{1}(D(\mathbf{t}))\right)$ satisfying $\operatorname{res}_{t_{i}}\left(\omega_{1}\right)=\lambda_{i}^{\prime}$ and $\operatorname{res}_{t_{i}}\left(\omega_{3}\right)$ $=\operatorname{res}_{t_{i}}\left(\nabla_{L^{\prime}}\right)-\lambda_{i}^{\prime}$ for $i=1, \ldots, n$. Take any member $\tau^{-1}\left(E, \nabla_{E}, \varphi,\left\{l_{j}\right\}\right) \in B$. Then there exists a subbundle $F \subset E$ satisfying $\nabla_{E}(F) \subset F \otimes \Omega_{\mathbf{P}^{1}}^{1}(D(\mathbf{t}))$ and $\operatorname{res}_{t_{i}}\left(\nabla_{F}\right)=\lambda_{i}^{\prime}$ for any $i$. Since $\sum_{i=1}^{n} \lambda_{i}^{\prime}=0, F \cong \mathcal{O}_{\mathbf{P}^{1}}$ and $E / F \cong \mathcal{O}_{\mathbf{P}^{1}}$. Thus we have $E \cong \mathcal{O}_{\mathbf{P}^{1}} \oplus \mathcal{O}_{\mathbf{P}^{1}}$ and $\nabla_{E}$ can be given by

$$
\nabla_{E}: \mathcal{O}_{\mathbf{P}^{1}} \oplus \mathcal{O}_{\mathbf{P}^{1}} \longrightarrow\left(\mathcal{O}_{\mathbf{P}^{1}} \oplus \mathcal{O}_{\mathbf{P}^{1}}\right) \otimes \Omega_{\mathbf{P}^{1}}^{1}(D(\mathbf{t}))
$$




$$
\left(\begin{array}{l}
f_{1} \\
f_{2}
\end{array}\right) \mapsto\left(\begin{array}{l}
d f_{1} \\
d f_{2}
\end{array}\right)+\left(\begin{array}{cc}
\omega_{1} & \omega_{2} \\
0 & \omega_{3}
\end{array}\right)\left(\begin{array}{l}
f_{1} \\
f_{2}
\end{array}\right)
$$

So put

$$
Z:=\left\{\begin{array}{l|l}
\left(\omega_{2},\left\{l_{i}\right\}_{i=1}^{n}\right) & \begin{array}{l}
\omega_{2} \in H^{0}\left(\Omega_{\mathbf{P}^{1}}^{1}(D(\mathbf{t}))\right) \text { and }\left.l_{i} \subset\left(\mathcal{O}_{\mathbf{P}^{1}} \oplus \mathcal{O}_{\mathbf{P}^{1}}\right)\right|_{t_{i}} \text { is a line } \\
\text { such that } l_{i}=\left.\left(\mathcal{O}_{\mathbf{P}^{1}} \oplus 0\right)\right|_{t_{i}} \text { for } i \text { satisfying res } t_{i}\left(\omega_{2}\right) \neq 0 \\
\text { or } 2 \lambda_{i}^{\prime} \notin \mathbf{Z} \text {, the parabolic connection } \tau^{-1}\left(\mathcal{O}_{\mathbf{P}^{1}} \oplus \mathcal{O}_{\mathbf{P}^{1}},\right. \\
\left.\nabla, \varphi,\left\{l_{i}\right\}\right) \text { is } \boldsymbol{\alpha} \text {-stable, where } \nabla \text { is given by } \nabla\left(f_{1}, f_{2}\right) \\
=\left(d f_{1}, d f_{2}\right)+\left(\omega_{1} f_{1}+\omega_{2} f_{2}, \omega_{3} f_{2}\right) \text { for } f_{1}, f_{2} \in \mathcal{O}_{\mathbf{P}^{1}} \text { and } \\
\varphi: \bigwedge^{2}\left(\mathcal{O}_{\mathbf{P}^{1}} \oplus \mathcal{O}_{\mathbf{P}^{1}} \stackrel{\sim}{\rightarrow} L^{\prime}\right.
\end{array}
\end{array}\right\}
$$

Then we obtain a morphism

$$
f: Z \longrightarrow B
$$

which is surjective.

Assume that $2 \lambda_{i} \notin \mathbf{Z}$ for some $i$. In this case, $\omega_{1}-\omega_{3} \neq 0$ and the group

$$
G=\left\{\left(\begin{array}{cc}
c & a \\
0 & c^{-1}
\end{array}\right) \mid c \in \mathbf{C}^{\times}, a \in \mathbf{C}\right\} /\left\{ \pm\left(\begin{array}{ll}
1 & 0 \\
0 & 1
\end{array}\right)\right\}
$$

acts freely on $Z \backslash\left\{\right.$ the locus $\left.\omega_{2} \in \mathbf{C}\left(\omega_{1}-\omega_{3}\right)\right\}$ satisfying $f(g x)=f(x)$ for $g \in G$ and $x \in Z$. Thus we can see that $\operatorname{dim} f\left(Z \backslash\left\{\right.\right.$ the locus $\left.\left.\omega_{2} \in \mathbf{C}\left(\omega_{1}-\omega_{3}\right)\right\}\right) \leq$ $\operatorname{dim} H^{0}\left(\Omega_{\mathbf{P}^{1}}^{1}(D(\mathbf{t}))\right)-2=n-3$. Take a point $t_{j}$ satisfying $\operatorname{res}_{t_{j}}\left(\omega_{1}-\omega_{3}\right) \neq 0$ and put

$$
\begin{aligned}
& Y:=\left\{\left(\omega_{2},\left\{l_{i}\right\}\right) \in Z \mid \omega_{2} \in \mathbf{C}\left(\omega_{1}-\omega_{3}\right)\right\} \\
& X:=\left\{\left(\omega_{2},\left\{l_{i}\right\}\right) \in Y \mid l_{i}=\mathbf{C}\left(\begin{array}{c}
\operatorname{res}_{t_{j}}\left(\omega_{2}\right) \\
\operatorname{res}_{t_{j}}\left(\omega_{3}-\omega_{1}\right)
\end{array}\right) \text { if } 2 \lambda_{i} \in \mathbf{Z} \text { and } \operatorname{res}_{t_{i}}\left(\omega_{2}\right) \neq 0\right\} .
\end{aligned}
$$

Then $\operatorname{dim} X \leq 1$ and $G$ acts freely on $Y \backslash X$. So we have $\operatorname{dim} f(Y) \leq n-3$. Thus we have $\operatorname{dim} B<2 n-6$.

Assume that $2 \lambda_{i} \in \mathbf{Z}$ for all $i$. Take any member $\tau^{-1}\left(E, \nabla_{E}, \varphi,\left\{l_{j}\right\}\right)$ of $B$. If $\operatorname{res}_{t_{i}}\left(\nabla_{E}\right) \neq \lambda_{i}^{\prime}$ id for any $i$, the object $\tau^{-1}\left(E, \nabla_{E}, \varphi,\left\{l_{j}\right\}\right)$ can not be stable for any choice of $\boldsymbol{\alpha}$ because it has a nontrivial endomorphism. Thus $\operatorname{res}_{t_{i}}\left(\nabla_{E}\right)=\lambda_{i}^{\prime}$ id for some $i$. Then we can check that $G$ acts freely on $Z$ and $\operatorname{dim} f(Z) \leq \operatorname{dim} Z-\operatorname{dim} G=n-3$. Since $M_{n}^{\boldsymbol{\alpha}}(\mathbf{t}, \boldsymbol{\lambda}, L) \backslash M_{n}^{\boldsymbol{\alpha}}(\mathbf{t}, \boldsymbol{\lambda}, L)^{\sharp}$ is a union of the subsets like $A_{i}, B$, we have $\operatorname{dim}\left(M_{n}^{\boldsymbol{\alpha}}(\mathbf{t}, \boldsymbol{\lambda}, L) \backslash M_{n}^{\boldsymbol{\alpha}}(\mathbf{t}, \boldsymbol{\lambda}, L)^{\sharp}\right)<2 n-6$. 


\section{$\S 10$. Properness of the Riemann-Hilbert Correspondence}

We set

$$
\mathcal{R}\left(\mathcal{P}_{n, \mathbf{t}}\right)_{\mathbf{a}}^{i r r}:=\left\{[\rho] \in \mathcal{R}\left(\mathcal{P}_{n, \mathbf{t}}\right)_{\mathbf{a}} \mid \rho \text { is irreducible }\right\} .
$$

For $\boldsymbol{\lambda} \in \Lambda_{n}$ such that $2 \cos 2 \pi \lambda_{i}=a_{i}$ for $i=1, \ldots, n$, we put

$$
M_{n}^{i r r}(\mathbf{t}, \boldsymbol{\lambda}, L):=\left\{p \in M_{n}^{\boldsymbol{\alpha}}(\mathbf{t}, \boldsymbol{\lambda}, L) \mid \mathbf{R H}(p) \in \mathcal{R}\left(\mathcal{P}_{n, \mathbf{t}}\right)_{\mathbf{a}}^{\text {irr }}\right\} .
$$

Lemma 10.1. The restriction $M_{n}^{\text {irr }}(\mathbf{t}, \boldsymbol{\lambda}, L) \stackrel{\mathbf{R H}}{\longrightarrow} \mathcal{R}\left(\mathcal{P}_{n, \mathbf{t}}\right)_{\mathbf{a}}^{i r r}$ is a proper morphism.

Proof. As in the proof of Lemma 7.1, we can obtain an isomorphism

$$
\tau: M_{n}^{i r r}(\mathbf{t}, \boldsymbol{\lambda}, L) \stackrel{\sim}{\longrightarrow} M_{n}^{i r r}\left(\mathbf{t}, \boldsymbol{\lambda}^{\prime}, L^{\prime}\right)
$$

by composing elementary transforms and other transforms, where $0 \leq \operatorname{Re}\left(\lambda_{i}^{\prime}\right)<$ 1 and $0 \leq \operatorname{Re}\left(\operatorname{res}_{t_{i}}\left(\nabla_{L^{\prime}}\right)-\lambda_{i}^{\prime}\right)<1$ for $i=1, \ldots, n$. Let $N_{n}^{i r r}\left(\mathbf{t}, \boldsymbol{\lambda}^{\prime}, L^{\prime}\right)$ be the moduli space of irreducible connections $\left(E, \nabla_{E}\right)$ with a horizontal isomorphism $\operatorname{det}(E) \cong L^{\prime}$ satisfying $\operatorname{det}\left(\operatorname{res}_{t_{i}}\left(\nabla_{E}\right)-\lambda_{i}^{\prime}\right)=0$ for $i=1, \ldots, n$. As in the proof of Lemma 7.1, we obtain a factorization

$$
\mathbf{R H}: M_{n}^{i r r}(\mathbf{t}, \boldsymbol{\lambda}, L) \stackrel{\sim}{\longrightarrow} M_{n}^{i r r}\left(\mathbf{t}, \boldsymbol{\lambda}^{\prime}, L^{\prime}\right) \stackrel{\pi}{\longrightarrow} N_{n}^{i r r}\left(\mathbf{t}, \boldsymbol{\lambda}^{\prime}, L^{\prime}\right) \stackrel{\sim}{\longrightarrow} \mathcal{R}\left(\mathcal{P}_{n, \mathbf{t}}\right)_{\mathbf{a}}^{i r r} .
$$

So it is sufficient to show that $\pi: M_{n}^{i r r}\left(\mathbf{t}, \boldsymbol{\lambda}^{\prime}, L^{\prime}\right) \rightarrow N_{n}^{i r r}\left(\mathbf{t}, \boldsymbol{\lambda}^{\prime}, L^{\prime}\right)$ is proper. Let $R$ be any discrete valuation ring and $K$ its quotient field. Assume that a commutative diagram

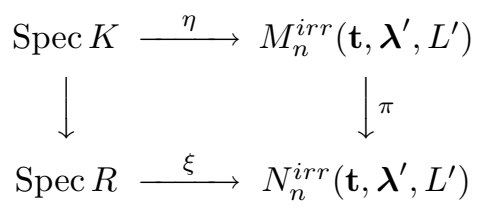

is given. Then $\xi$ corresponds to a flat family of connections $\left(E, \nabla_{E}, \varphi\right)$ on $\mathbf{P}_{R}^{1}$ over $R$ and $\eta$ corresponds to parabolic structures $\left\{\left.l_{j} \subset(E \otimes K)\right|_{t_{j} \otimes K}\right\}$ satisfying $\left.\left(\operatorname{res}_{t_{j} \otimes K}\left(\nabla_{E} \otimes K\right)-\lambda_{j}^{\prime}\right)\right|_{l_{j}}=0$. We can take subbundles $\tilde{l}_{j} \subset$ $\left.E\right|_{t_{j} \otimes R}$ such that $\tilde{l}_{j} \otimes K=l_{j}$ as subspaces of $\left.(E \otimes K)\right|_{t_{j} \otimes K}$. Then we have $\left.\left(\operatorname{res}_{t_{j} \otimes R}\left(\nabla_{E}\right)-\lambda_{j}^{\prime}\right)\right|_{\tilde{l}_{j}}=0$ and $\left(E, \nabla_{E}, \varphi,\left\{l_{j}\right\}\right)$ becomes an $R$-valued point of $M_{n}^{i r r}\left(\mathbf{t}, \boldsymbol{\lambda}^{\prime}, L^{\prime}\right)$ whose image by $\pi$ is $\xi$ and the restriction to Spec $K$ is $\eta$. Thus $\pi: M_{n}^{i r r}\left(\mathbf{t}, \lambda^{\prime}, L^{\prime}\right) \rightarrow N_{n}^{i r r}\left(\mathbf{t}, \lambda^{\prime}, L^{\prime}\right)$ becomes a proper morphism by the valuative criterion of properness. 
Put

$$
\begin{aligned}
\mathcal{R}\left(\mathcal{P}_{n, \mathbf{t}}\right)_{\mathbf{a}}^{r e d} & :=\mathcal{R}\left(\mathcal{P}_{n, \mathbf{t}}\right)_{\mathbf{a}} \backslash \mathcal{R}\left(\mathcal{P}_{n, \mathbf{t}}\right)_{\mathbf{a}}^{i r r} \\
M_{n}^{r e d}(\mathbf{t}, \boldsymbol{\lambda}, L) & :=M_{n}^{\boldsymbol{\alpha}}(\mathbf{t}, \boldsymbol{\lambda}, L) \backslash M_{n}^{i r r}(\mathbf{t}, \boldsymbol{\lambda}, L) .
\end{aligned}
$$

Lemma 10.2. $\quad M_{n}^{\text {red }}(\mathbf{t}, \boldsymbol{\lambda}, L)$ is a closed subset of $M_{n}^{\boldsymbol{\alpha}}(\mathbf{t}, \boldsymbol{\lambda}, L)$ which is proper over $\mathbf{C}$.

Proof. First we will show that $M_{n}^{r e d}(\mathbf{t}, \boldsymbol{\lambda}, L)$ is a constructible subset of $M_{n}^{\boldsymbol{\alpha}}(\mathbf{t}, \boldsymbol{\lambda}, L)$. Take any member $\left(E, \nabla_{E}, \varphi,\left\{l_{i}\right\}\right)$ of $M_{n}^{\boldsymbol{\alpha}}(\mathbf{t}, \boldsymbol{\lambda}, L)$. Then

$\left(E, \nabla_{E}, \varphi,\left\{l_{i}\right\}\right) \in M_{n}^{r e d}(\mathbf{t}, \boldsymbol{\lambda}, L)$

$\Leftrightarrow \exists F \subset E$ : subbundle of rank 1 such that $\nabla_{E}(F) \subset F \otimes \Omega_{\mathbf{P}^{1}}^{1}(D(\mathbf{t}))$.

Note that such subbundles $F$ must satisfy

$$
\operatorname{res}_{t_{i}}\left(\left.\nabla_{E}\right|_{F}\right)=\lambda_{i} \text { or } \operatorname{res}_{t_{i}}\left(\left.\nabla_{E}\right|_{F}\right)=\operatorname{res}_{t_{i}}\left(\nabla_{L}\right)-\lambda_{i} \quad \text { for } i=1, \ldots, n \text {, }
$$

from which the choice of isomorphism classes of $\left(F,\left.\nabla_{E}\right|_{F}\right)$ must be finite. Let $L_{1}, L_{2}, \ldots, L_{m}$ be all the line bundles with connections satisfying the condition (184). Then we can see that the set

$$
\left\{\begin{array}{l|l}
\left(p, L_{i} \stackrel{\iota}{\rightarrow} E\right) \mid \begin{array}{l}
p=\left(E, \nabla_{E}, \varphi,\left\{l_{i}\right\}\right) \in M_{n}^{\boldsymbol{\alpha}}(\mathbf{t}, \boldsymbol{\lambda}, L) \text { and } \iota \text { is an injective } \\
\text { homomorphism such that } \nabla_{E}\left(\iota\left(L_{i}\right)\right) \subset \iota\left(L_{i}\right) \otimes \Omega_{\mathbf{P}^{1}}^{1}(D(\mathbf{t}))
\end{array}
\end{array}\right\}
$$

can be parameterized by a scheme of finite type over $M_{n}^{\boldsymbol{\alpha}}(\mathbf{t}, \boldsymbol{\lambda}, L)$. Thus $M_{n}^{r e d}(\mathbf{t}, \boldsymbol{\lambda}, L)$ is a constructible subset of $M_{n}^{\boldsymbol{\alpha}}(\mathbf{t}, \boldsymbol{\lambda}, L)$.

So it suffices to show by [H], Chapter II, Lemma 4.5 that $M_{n}^{r e d}(\mathbf{t}, \boldsymbol{\lambda}, L)$ is stable under specialization in the compactification $\overline{M_{n}^{\boldsymbol{\alpha}^{\prime} \boldsymbol{\beta}}}(\mathbf{t}, \boldsymbol{\lambda}, L)$. Take any scheme point $x_{1} \in M_{n}^{r e d}(\mathbf{t}, \boldsymbol{\lambda}, L)$ and $T$ be the closure of $\left\{x_{1}\right\}$ in $\overline{M_{n}^{\boldsymbol{\alpha}^{\prime} \boldsymbol{\beta}}}(\mathbf{t}, \boldsymbol{\lambda}, L)$. Take any point $x_{0} \in T$. Put $K:=k\left(x_{1}\right)$. Then there exists a discrete valuation ring $R$ with quotient field $K$ which dominates $\mathcal{O}_{x_{0}}$. A morphism $\iota: \operatorname{Spec} R \rightarrow$ $\overline{M_{n}^{\boldsymbol{\alpha}^{\prime} \boldsymbol{\beta}}}(\mathbf{t}, \boldsymbol{\lambda}, L)$ satisfying $\iota(\eta)=x_{1}$ and $\iota(\xi)=x_{0}$ is induced, where $\eta$ is the generic point of $\operatorname{Spec} R$ and $\xi$ the closed point of $\operatorname{Spec} R$. $\iota$ corresponds to a flat family of $(\mathbf{t}, \boldsymbol{\lambda})$-parabolic $\phi$-connections $\left(\tilde{E}_{1}, \tilde{E}_{2}, \tilde{\phi}, \tilde{\nabla}, \tilde{\varphi},\left\{\tilde{l}_{i}\right\}\right)$ on $\mathbf{P}_{R}^{1}$ over R. Put

$$
\begin{aligned}
\left(E_{1}, E_{2}, \phi, \nabla, \varphi,\left\{l_{i}\right\}\right) & :=\left(\tilde{E}_{1}, \tilde{E}_{2}, \tilde{\phi}, \tilde{\nabla}, \tilde{\varphi},\left\{\tilde{l}_{i}\right\}\right) \otimes k(\eta) \\
\left(E_{1}^{\prime}, E_{2}^{\prime}, \phi^{\prime}, \nabla^{\prime}, \varphi^{\prime},\left\{l_{i}^{\prime}\right\}\right) & :=\left(\tilde{E}_{1}, \tilde{E}_{2}, \tilde{\phi}, \tilde{\nabla}, \tilde{\varphi},\left\{\tilde{l}_{i}\right\}\right) \otimes k(\xi),
\end{aligned}
$$


Since $\left(E_{1}, E_{2}, \phi, \nabla, \varphi,\left\{l_{i}\right\}\right) \in M_{n}^{r e d}(\mathbf{t}, \boldsymbol{\lambda}, L)(K), \phi$ is isomorphic and there exist commutative diagrams

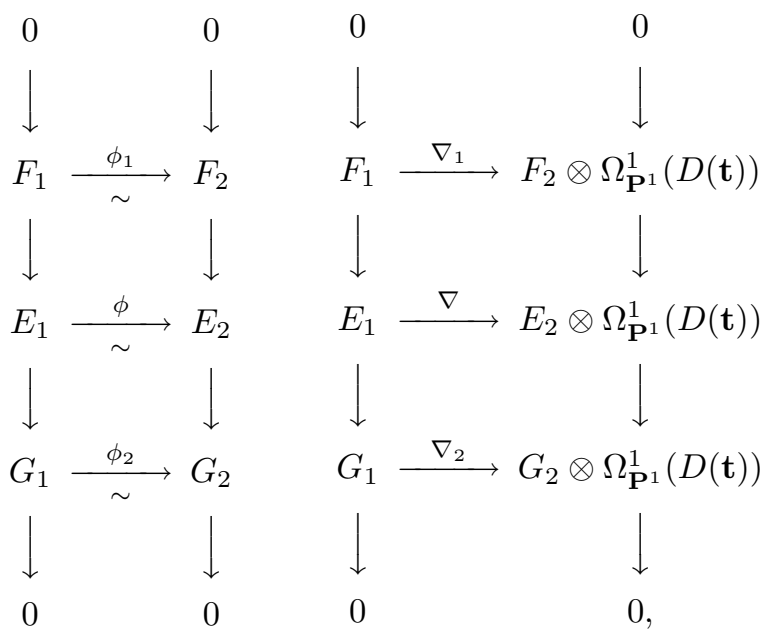

where $F_{1}, F_{2}, G_{1}, G_{2}$ are line bundles. There exist quotient coherent sheaves $\tilde{E}_{1} \rightarrow \tilde{G}_{1}, \tilde{E}_{2} \rightarrow \tilde{G}_{2}$ which are flat over $R$ and whose fibers over $\eta$ are isomorphic to $E_{1} \rightarrow G_{1}$ and $E_{2} \rightarrow G_{2}$, respectively. Put $\tilde{F}_{1}:=\operatorname{ker}\left(\tilde{E}_{1} \rightarrow \tilde{G}_{1}\right)$ and $\tilde{F}_{2}:=\operatorname{ker}\left(\tilde{E}_{2} \rightarrow \tilde{G}_{2}\right)$. Then we obtain commutative diagrams

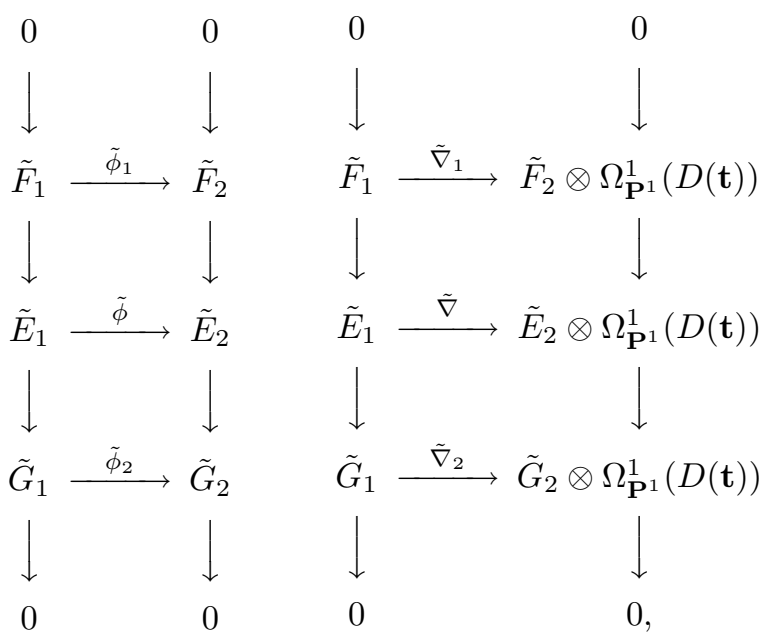

whose fibers over $\eta$ are the commutative diagrams (185). Put $G_{1}^{\prime}:=$ $\tilde{G}_{1}(\xi) /\left(\tilde{G}_{1}(\xi)\right)_{t o r}, G_{2}^{\prime}:=\tilde{G}_{2}(\xi) /\left(\tilde{G}_{2}(\xi)\right)_{t o r}, F_{1}^{\prime}:=\operatorname{ker}\left(\tilde{E}_{1}(\xi) \rightarrow G_{1}^{\prime}\right)$ and $F_{2}^{\prime}:=$ $\operatorname{ker}\left(\tilde{E}_{2}(\xi) \rightarrow G_{2}^{\prime}\right)$, where $\left(\tilde{G}_{1}(\xi)\right)_{\text {tor }}$ and $\left(\tilde{G}_{1}(\xi)\right)_{\text {tor }}$ are the torsion parts of 
$\tilde{G}_{1}(\xi)$ and $\tilde{G}_{2}(\xi)$, respectively. Then we obtain commutative diagrams

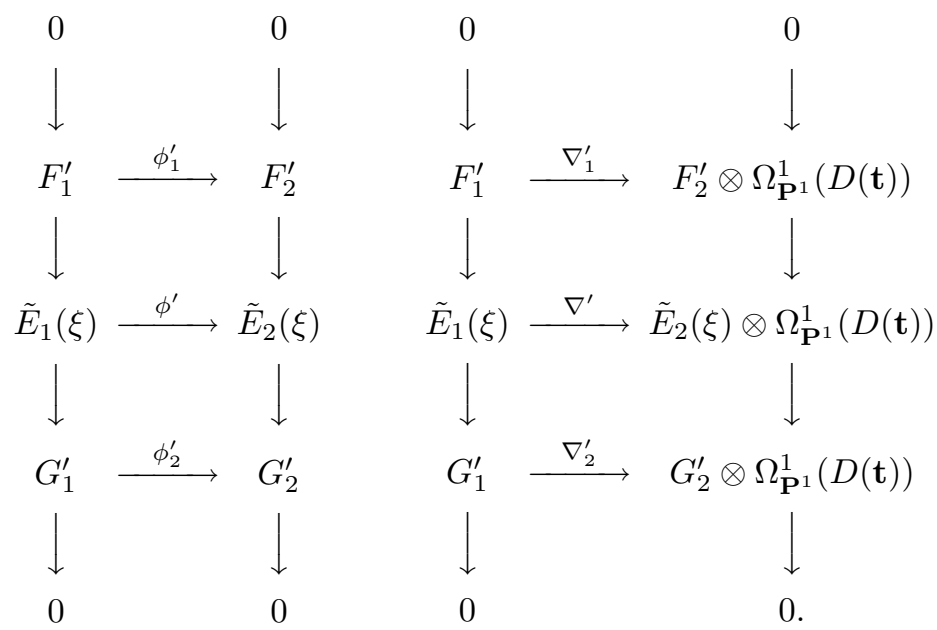

Assume that $x_{0} \notin M_{n}^{\boldsymbol{\alpha}}(\mathbf{t}, \boldsymbol{\lambda}, L)$. Then we have either

$$
\left\{\begin{array}{l}
\phi_{1}^{\prime}=0 \text { and } \operatorname{deg} F_{1}^{\prime} \geq \operatorname{deg} F_{2}^{\prime} \text { or } \\
\phi_{2}^{\prime}=0 \text { and } \operatorname{deg} G_{1}^{\prime} \geq \operatorname{deg} G_{2}^{\prime} .
\end{array}\right.
$$

Assume $\phi_{1}^{\prime}=0$ and $\operatorname{deg} F_{1}^{\prime} \geq \operatorname{deg} F_{2}^{\prime}$. Then $\operatorname{res}_{t_{i}}\left(\nabla_{1}^{\prime}\right)=\lambda_{i}^{\prime} \phi_{1}^{\prime}\left(t_{i}\right)=0$ for $i=1, \ldots, n$, where $\lambda_{i}^{\prime}=\lambda_{i}$ or $\lambda_{i}^{\prime}=\operatorname{res}_{t_{i}}\left(\nabla_{L}\right)-\lambda_{i}$. We must have $\nabla_{1}^{\prime}=0$, since $H^{0}\left(\mathbf{P}^{1},\left(F_{1}^{\prime}\right)^{\vee} \otimes F_{2}^{\prime} \otimes \Omega_{\mathbf{P}^{1}}^{1}\right)=0$. Then $\left(F_{1}^{\prime}, 0\right)$ collapses the stability of $\left(\tilde{E}_{1}(\xi), \tilde{E}_{2}(\xi), \tilde{\phi}(\xi), \tilde{\nabla}(\xi), \tilde{\varphi}(\xi),\left\{\tilde{l}_{i}(\xi)\right\}\right)$. Assume $\phi_{2}^{\prime}=0$ and $\operatorname{deg} G_{1}^{\prime} \geq \operatorname{deg} G_{2}^{\prime}$. Then $\operatorname{res}_{t_{i}}\left(\nabla_{2}^{\prime}\right)=\lambda_{i}^{\prime} \phi_{2}^{\prime}\left(t_{i}\right)=0$ for $i=1, \ldots, n$, where $\lambda_{i}^{\prime}=\lambda_{i}$ or $\lambda_{i}^{\prime}=$ $\operatorname{res}_{t_{i}}\left(\nabla_{L}\right)-\lambda_{i}$. Again we have $\nabla_{2}^{\prime}=0$, because $\operatorname{deg} G_{1}^{\prime} \geq \operatorname{deg} G_{2}^{\prime}$. Then $\left(\tilde{E}_{1}(\xi), F_{2}^{\prime}\right)$ collapses the stability of $\left(\tilde{E}_{1}(\xi), \tilde{E}_{2}(\xi), \tilde{\phi}(\xi), \tilde{\nabla}(\xi), \tilde{\varphi}(\xi),\left\{\tilde{l}_{i}(\xi)\right\}\right)$. Hence we must have $x_{0} \in M_{n}^{\boldsymbol{\alpha}}(\mathbf{t}, \boldsymbol{\lambda}, L)$ and so $x_{0} \in M_{n}^{\text {red }}(\mathbf{t}, \boldsymbol{\lambda}, L)$.

Now we are ready to prove the following

Proposition 10.1. The analytic morphism

$$
\mathbf{R H}_{\mathbf{t}, \boldsymbol{\lambda}}: M_{n}^{\boldsymbol{\alpha}}(\mathbf{t}, \boldsymbol{\lambda}, L) \longrightarrow \mathcal{R}_{n}\left(\mathcal{P}_{n, \mathbf{t}}\right)_{\mathbf{a}}
$$

defined in (161) is proper.

Proof. Recall that we have proved the assertions in Theorem 7.1 except for the properness of $\mathbf{R H}_{\mathbf{t}, \boldsymbol{\lambda}}$. Therefore, we see that $\mathbf{R} \mathbf{H}_{\mathbf{t}, \boldsymbol{\lambda}}$ induces the an analytic isomorphism

$$
M_{n}^{\boldsymbol{\alpha}}(\mathbf{t}, \boldsymbol{\lambda}, L)^{\sharp} \stackrel{\simeq}{\longrightarrow} \mathcal{R}\left(\mathcal{P}_{n, \mathbf{t}}\right)_{\mathbf{a}}^{\sharp} .
$$


(See (164)). From the third assertion of Theorem 7.1, we see that $\mathcal{R}\left(\mathcal{P}_{n, \mathbf{t}}\right)_{\mathbf{a}}^{\text {sing }}=$ $\mathcal{R}\left(\mathcal{P}_{n, \mathbf{t}}\right)_{\mathbf{a}} \backslash \mathcal{R}\left(\mathcal{P}_{n, \mathbf{t}}\right)_{\mathbf{a}}^{\sharp}$ has codimension $\geq 2$ in $\mathcal{R}\left(\mathcal{P}_{n, \mathbf{t}}\right)_{\mathbf{a}}$. Moreover Lemma 10.1 and Lemma 10.2 shows that every fiber of $\mathbf{R H}_{\mathbf{t}, \boldsymbol{\lambda}}$ at each closed point of $\mathcal{R}_{n}\left(\mathcal{P}_{n, \mathbf{t}}\right)_{\mathbf{a}}$ is compact. Therefore the assertion follows from the following lemma due to A. Fujiki.

Lemma 10.3. Let $f: X \rightarrow Y$ be a surjective holomorphic mapping of irreducible analytic varieties. Assume that an analytic closed subset $S$ of $Y$ exists such that $\operatorname{codim}_{Y} S \geq 2, X^{\sharp}:=f^{-1}\left(Y^{\sharp}\right)$ is dense in $X$, where $Y^{\sharp}=Y \backslash S$ and that the restriction $\left.f\right|_{X^{\sharp}}: X^{\sharp} \rightarrow Y^{\sharp}$ is an analytic isomorphism. Moreover assume that the fibers $f^{-1}(y)$ are compact for all $y \in Y$. Then $f$ is a proper mapping.

Proof. Since normalization morphism is proper, we may assume that both $X$ and $Y$ are normal by replacing them by their normalizations. Take any point $y \in Y$. Since $f^{-1}(y)$ is compact, there is an open neighborhood $U$ of $f^{-1}(y)$ and $V$ of $y$ such that $f(U) \subset V$ and the restriction $\left.f\right|_{U}: U \rightarrow V$ is a proper mapping. Since $Y^{\sharp}$ is normal, we may assume that $Y^{\sharp} \cap V$ is connected. $f\left(X^{\sharp} \cap U\right)$ is open in $Y^{\sharp} \cap V$, because $X^{\sharp} \stackrel{f}{\longrightarrow} Y^{\sharp}$ is an isomorphism. $f\left(X^{\sharp} \cap U\right)$ is also closed in $Y^{\sharp} \cap V$, because $X^{\sharp} \cap U \stackrel{f}{\longrightarrow} Y^{\sharp} \cap V$ is proper. Thus we have $f\left(X^{\sharp} \cap U\right)=Y^{\sharp} \cap V$, because $X^{\sharp}$ is dense in $X$.

Assume that $f^{-1}(V) \backslash U \neq \emptyset$ and take a point $b \in f^{-1}(V) \backslash U$. Take an open neighborhood $W$ of $b$ such that $f(W) \subset V . X^{\sharp} \cap W$ is nonempty and dense in $W$. From the injectivity of $\left.f\right|_{X^{\sharp}}$ and the fact $f\left(X^{\sharp} \cap U\right)=Y^{\sharp} \cap V$, we have $X^{\sharp} \cap W \subset X^{\sharp} \cap U$, since $f\left(X^{\sharp} \cap W\right) \subset Y^{\sharp} \cap V$. Taking closures in $W$, we have $b \in W \subset U$, which is a contradiction. Thus we have $f^{-1}(V)=U$. 


\section{$\S 11$. List of Notation}

Notation

$T_{n}$

$\Lambda_{n}$

$D(\mathbf{t})$

$\operatorname{pardeg}_{\alpha} E$

$\underline{M_{n}^{\alpha}(\mathbf{t}, \lambda, L)}$

$\overline{M_{n}^{\boldsymbol{\alpha}^{\prime} \boldsymbol{\beta}}}(\mathbf{t}, \boldsymbol{\lambda}, L)$

$\bar{\pi}_{n}: \overline{\mathcal{M}}_{n}^{\boldsymbol{\alpha}^{\prime} \boldsymbol{\beta}}(L) \longrightarrow T_{n} \times \Lambda_{n}$

$\pi_{n}: \mathcal{M}_{n}^{\alpha}(L) \longrightarrow T_{n} \times \Lambda_{n}$

$E l m_{t_{i}}^{+}: M_{n}^{\boldsymbol{\alpha}}(\mathbf{t}, \boldsymbol{\lambda}, L) \longrightarrow M_{n}^{\boldsymbol{\alpha}}\left(\mathbf{t}, \boldsymbol{\lambda}^{\prime}, L\left(t_{i}\right)\right)$

$E l m_{t_{i}}^{-}: M_{n}^{\boldsymbol{\alpha}}(\mathbf{t}, \boldsymbol{\lambda}, L) \longrightarrow M_{n}^{\boldsymbol{\alpha}}\left(\mathbf{t}, \boldsymbol{\lambda}^{\prime}, L\left(-t_{i}\right)\right)$

$R_{i}(E)$

$B L_{n}$

$\mathcal{R}\left(\mathcal{P}_{n, \mathbf{t}}\right)=\operatorname{Spec}\left[\left(R_{n-1}\right)^{\operatorname{Ad}\left(S L_{2}(\mathbf{C})\right)}\right]$

$T_{n}^{\prime}:=\tilde{T}_{n} / \Gamma_{n-1} \longrightarrow T_{n}$

$\phi_{n}: \mathcal{R}_{n} \longrightarrow T_{n}^{\prime} \times \mathcal{A}_{n}$

$\mathcal{R}\left(\mathcal{P}_{n, \mathbf{t}}\right)_{\mathbf{a}}$

$\mathcal{F}^{0}$

$\mathcal{F}^{1}$

$\mathcal{F}^{1,+}$

$\mathcal{F}^{\bullet}$

$\mathcal{F}^{\bullet},+$

$\Omega \in H^{0}\left(M_{n}^{\alpha}, \Omega_{M_{n}^{\alpha} / T_{n}^{\prime} \times \Lambda_{n}}^{2}\right)$

$\Omega_{1} \in \Gamma\left(\mathcal{R}_{n}^{\sharp}, \Omega_{\mathcal{R}_{n}^{\sharp} / T_{n}^{\prime} \times \mathcal{A}_{n}}^{2}\right)$

$\mathbf{R H}_{\mathbf{t}, \boldsymbol{\lambda}}: M_{n}^{\boldsymbol{\alpha}}(\mathbf{t}, \boldsymbol{\lambda}, L) \longrightarrow \mathcal{R}\left(\mathcal{P}_{n, \mathbf{t}}\right)_{\mathbf{a}}$

$\mathbf{R H}_{n}: M_{n}^{\alpha}(L) \longrightarrow \mathcal{R}_{n}$

$\mu_{n}$

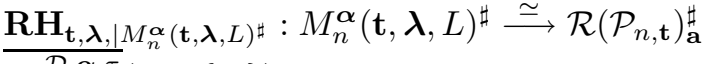

$$
\begin{aligned}
& \overline{\mathcal{M}_{\mathcal{X} / S}^{\mathcal{D}, \boldsymbol{\alpha}, \tau}}\left(r, d,\left\{d_{i}\right\}\right)
\end{aligned}
$$

number of page
equation

(32) $\quad 1010$

(33) 1010

(34) 1010

(39) 1012

(42) 1013

(43) 1014

(48) 1015

(48) 1015

(56) 1018

(57) 1018

(70) 1020

(76) 1022

(88) 1025

(94) 1027

(96) 1027

(97) 1027

(111) 1054

(112) 1054

(113) 1054

(114) 1055

(115) 1055

(125) 1057

(154) 1065

(161) 1068

(162) 1068

(163) 1068

(164) 1068

(104) $\quad 1030$

\section{References}

[A] Arinkin, D., Orthogonality of natural sheaves on moduli stacks of SL(2)-bundles with connections on $\mathbf{P}^{1}$ minus 4 points, Selecta Math. (N.S.), 7 (2001), 213-239.

[AL1] Arinkin, D. and Lysenko, S., On the moduli of SL(2)-bundles with connections on $\mathbf{P}^{1} \backslash\left\{t_{1}, \ldots, t_{4}\right\}$. Internat. Math. Res. Notices, 1997, no. 19, 983-999. 
[AL2] Arinkin, D. and Lysenko, S., Isomorphisms between moduli spaces of SL(2)-bundles with connections on $\mathbf{P}^{1} \backslash\left\{x_{1}, \ldots, x_{4}\right\}$. Math. Res. Lett., 4 (1997), 181-190.

[Bea] Beauville, A., Symplectic singularities, Invent. Math., 139 (2000), 541-549.

[Boa] Boalch, P., From Klein to Painlevé via Fourier, Laplace and Jimbo, Proc. London Math. Soc. (3), 90 (2005), 167-208.

[Dek] Dekkers, W., The matrix of a connection having regular singularities on a vector bundle of rank 2 on $P^{1}(C)$, Équations différentielles et systèmes de Pfaff dans le champ complexe, Sem., Inst. Rech. Math. Avancée, Strasbourg, 1975, 33-43, Lecture Notes in Math., 712, Springer, Berlin, 1979.

[Del70] Deligne, P., Équations différentielles à points singuliers réguliers, Lecture Notes in Math., 163, Springer-Verlag, Berlin, 1970.

[DM] Dubrovin, B. and Mazzocco, M., Monodromy of certain Painlevé-VI transcendents and reflection groups, Invent. Math., 141 (2000), 55-147.

[FA] Fokas, A. S. and Ablowitz, M. J., On a unified approach to transformations and elementary solutions of Painlevé equations, J. Math. Phys., 23 (1982), 2033-2042.

[For] Formanek, E., The invariants of $n \times n$ matrices, Invariant Theory (Koh, S. S., eds.), 18-43, Lecture Notes in Math. 1278, Springer Verlag, 1987.

[F] Fuchs, R., Über lineare homogene Differentialgleichungen zweiter Ordnung mit drei im Endlichen gelegenen wesentlich singulären Stellen, Math. Ann., 63 (1907), 301-321.

[Ga] Garnier, R., Sur les equations differentielles du troisieme ordre dont l'integrale generale est uniforme et sur une classe d'equations nouvelles d'ordre superieur dont l'integrale generale a ses points critiques fixes, Ann. Ecole Norm. Sup., 29 (1912), 1-126.

[Go] Goldman, W. M., The symplectic nature of fundamental groups of surfaces, Adv. in Math., 54 (1984), 200-225.

[H] Hartshorne, R., Algebraic geometry, Graduate Texts in Mathematics, 52, SpringerVerlag, New York-Heidelberg, 1977.

[HL] Huybrechts, D. and Lehn, M., The geometry of moduli spaces of sheaves, Aspects of Mathematics, E31. Friedr. Vieweg \& Sohn, Braunschweig, 1997.

[In] Inaba, M., Moduli of parabolic stable sheaves on a projective scheme, J. Math. Kyoto Univ., 40 (2000), 119-136.

[IIS0] Inaba, M., Iwasaki, K. and Saito, M.-H., Bäcklund transformations of the sixth Painlevé equation in terms of Riemann-Hilbert correspondence, Int. Math. Res. Not., (2004), 1-30.

[IIS2] _ Moduli of Stable Parabolic Connections, Riemann-Hilbert correspondence and Geometry of Painlevé equation of type VI, II, to appear in Advanced Studies in Pure Math., 42, 2006, Moduli spaces and Arithmetic Geometry (Kyoto, 2004).

[IISA] — Dynamics of the sixth Painlevé Equations, to appear in Proceedings of International Conference on Asymptotic Theories and Painlevé Equations, Université d'Angers, 2004, Séminaires et Congrès, (math.AG/0501007).

[Iw1] Iwasaki, K., Moduli and deformation for Fuchsian projective connections on a Riemann surface, J. Fac. Sci. Univ. Tokyo Sect. IA Math., 38 (1991), 431-531.

[Iw2] Wuchsian moduli on a Riemann surface-its Poisson structure and Poincare'-Lefschetz duality, Pacific J. Math., 155 (1992), 319-340.

[Iw3] , A modular group action on cubic surfaces and the monodromy of the Painlevé VI equation, Proc. Japan Acad. Ser. A Math. Sci., 78 (2002), 131-135.

[Iw4] An area-preserving action of the modular group on cubic surfaces and the Painlevé VI equation, Comm. Math. Phys., 242 (2003), 185-219.

[J] Jimbo, M., Monodromy problem and the boundary condition for some Painlevé equations, Publ. RIMS, Kyoto Univ., 18, (1982), 1137-1161.

[JMU] Jimbo, M., Miwa, T. and Ueno, K., Monodromy preserving deformation of linear ordinary differential equations with rational coefficients. I. General theory and $\tau$ function, Phys. D, 2 (1981), 306-352. 
[JM] Jimbo, M. and Miwa, T., Monodromy preserving deformation of linear ordinary differential equations with rational coefficients. II., Phys. D, 2 (1981), 407-448.

[K] Kimura, H., Uniform foliation associated with the Hamiltonian system $\mathbf{H}_{n}$, Ann. Scuola Norm. Sup. Pisa Cl. Sci., (4), 20 (1993), 1-60.

[KM] Kollár, J. and Mori, S., Birational geometry of algebraic varieties, Cambridge Tracts in Mathematics, 134, Cambridge University Press, Cambridge, 1998.

[LY] Lukashevich, N. A. and Yablonski, A. I., On a class of solutions of the sixth Painlevé equations, Differential Equations, 3 (1967), 264-267.

[Mal] Malgrange, B., Sur les déformation isomonodromiques. I. singularités régulières, Mathématique et Physique (Paris, 1979/1982), 401-426, Progr. in Math., 37, Birkhäuser, Boston, 1983.

[M] Maruyama, M., Moduli of stable sheaves, II, J. Math. Kyoto Univ., 18 (1978), 557-614.

[MY] Maruyama, M. and Yokogawa, K., Moduli of parabolic stable sheaves, Math. Ann., 293 (1992), 77-99.

[Mal] Malmquist, J., Sur les équations différentielles du second ordre, dont l'intégrales générales a ses points critiques fixes, Arkiv. Math. Astr. Fys., 17 (1923), 1-89.

[MMT] Matano, T., Matumiya, A. and Takano, K., On some Hamiltonian structures of Painlevé systems, II, J. Math. Soc. Japan, 51 (1999), 843-866.

[Miw] Miwa, T., Painlevé property of monodromy preserving deformations and the analyticity of $\tau$ functions, Publ. RIMS, Kyoto Univ., 17 (1981), 709-721.

[Mum] Mumford, D., Geometric invariant theory, Ergebnisse der Mathematik und ihrer Grenzgebiete, Neue Folge, Band 34, Springer-Verlag, Berlin, 1965.

[N] Nakajima, H., Hyper-Kähler structures on moduli spaces of parabolic Higgs bundles on Riemann surfaces, Moduli of vector bundles (Sanda, 1994; Kyoto, 1994), 199208, Lecture Notes in Pure and Appl. Math., 179, Dekker, New York, 1996.

[Ni] Nitsure, N., Moduli of semistable logarithmic connections, J. Amer. Math. Soc., 6 (1993), 597-609.

[NY] Noumi, M. and Yamada, Y., A new Lax pair for the sixth Painlevé equation associated with $\hat{s o}(8)$, Microlocal analysis and complex Fourier analysis (Kawai, T. and Fujita, K. eds.), 238-252, World Scientific Publ., New Jersey, 2002.

[O1] Okamoto, K., Sur les feuilletages associés aux équations du second ordre à points critiques fixes de P. Painlevé, Espaces des conditions initiales, Japan. J. Math., 5 (1979), 1-79.

[O2] - Polynomial Hamiltonians associated with Painlevé equations, I, Proc. Japan Acad. Ser. A Math. Sci., 56 (1980), 264-268; II, ibid., 367-371.

[O3] - Isomonodromic deformation and Painlevé equations and the Garnier system, J. Fac. Sci. Univ. Tokyo Sect. IA Math., 33 (1986), 575-618

[O4] Study of the Painlevé equations I, sixth Painlevé equation $P_{V I}$, Ann. Math. Pura Appl. (4) 146 (1987), 337-381.

[P] Procesi, C., The invariant theory of $n \times n$ matrices, Adv. in Math., 19 (1976), 306-381.

[STT] Saito, M.-H., Takebe, T. and Terajima, H., Deformation of Okamoto-Painlevé pairs and Painlevé equations, J. Algebraic Geom., 11 (2002), 311-362.

[SU] Saito, M.-H. and Umemura, H., Painlevé equations and deformations of rational surfaces with rational double points. Physics and combinatorics 1999 (Nagoya), 320-365, World Sci. Publishing, River Edge, NJ, 2001.

[STa] Saito, M-.H. and Takebe, T., Classification of Okamoto-Painlevé Pairs, Kobe J. Math., 19 (2002), 21-55.

[STe] Saito, M-. H. and Terajima, H., Nodal curves and Riccati solutions of Painlevé equations, J. Math. Kyoto Univ., 44 (2004), 529-568.

[Sakai] Sakai, H., Rational surfaces associated with affine root systems and geometry of the Painlevé equations, Comm. Math. Phys., 220 (2001), 165-229.

[Sch] Schlesinger, L., Über eine Klasse von Differentialsystemen beliebliger Ordnung mit 
festen Kritischer Punkten, J. Reine Angew. Math., 141 (1912), 96-145.

[ST] Shioda, T. and Takano, K., On some Hamiltonian structures of Painlevé systems I, Funkcial. Ekvac., 40 (1997), 271-291.

[Sim1] Simpson, Carlos T., Moduli of representations of the fundamental group of a smooth projective variety. I, Inst. Hautes Études Sci. Publ. Math., 79 (1994), $47-129$.

[Sim2] Moduli of representations of the fundamental group of a smooth projective variety. II, Inst. Hautes Études Sci. Publ. Math., 80 (1994), 5-79 (1995).

[T] Terajima, H., On the space of monodromy data of Painlevé VI, Preprint, Kobe (2003).

[W] Watanabe, H., Birational canonical transformations and classical solutions of the sixth Painlevé equation, Ann. Scoula Norm. Sup. Pisa Cl. Sci. (4), 27 (1998), $379-425$. 